\title{
Weight training : Implications for energy metabolism
}

Citation for published version (APA):

van Etten, L. M. L. A. (1997). Weight training : Implications for energy metabolism. [Doctoral Thesis, Maastricht University, Maastricht University]. Universiteit Maastricht.

https://doi.org/10.26481/dis.19970228le

Document status and date:

Published: 01/01/1997

DOI:

10.26481/dis.19970228le

Document Version:

Publisher's PDF, also known as Version of record

\section{Please check the document version of this publication:}

- A submitted manuscript is the version of the article upon submission and before peer-review. There can be important differences between the submitted version and the official published version of record.

People interested in the research are advised to contact the author for the final version of the publication, or visit the DOI to the publisher's website.

- The final author version and the galley proof are versions of the publication after peer review.

- The final published version features the final layout of the paper including the volume, issue and page numbers.

Link to publication

\footnotetext{
General rights rights.

- You may freely distribute the URL identifying the publication in the public portal. please follow below link for the End User Agreement:

www.umlib.nl/taverne-license

Take down policy

If you believe that this document breaches copyright please contact us at:

repository@maastrichtuniversity.nl

providing details and we will investigate your claim.
}

Copyright and moral rights for the publications made accessible in the public portal are retained by the authors and/or other copyright owners and it is a condition of accessing publications that users recognise and abide by the legal requirements associated with these

- Users may download and print one copy of any publication from the public portal for the purpose of private study or research.

- You may not further distribute the material or use it for any profit-making activity or commercial gain

If the publication is distributed under the terms of Article $25 \mathrm{fa}$ of the Dutch Copyright Act, indicated by the "Taverne" license above, 
WEIGHT TRAINING: IMPLICATIONS FOR ENERGY METABOLISM 
Etten van, Ludovicus Maria Leonardus Anna /

Weight training: implications for energy metabolism

Copyright $\odot 1997$ by L.M.L.A. van Etten

ISBN $90-9010276-0$

Text Design and Layout: Ludo van Etten

Cover Design: Datawyse Maastricht: Mariëlle Retz

Photographer (cover): Ludo van Etten

Printed by: Datawyse Maastricht / Krips Repro Meppel 


\section{WEIGHT TRAINING: IMPLICATIONS FOR ENERGY METABOLISM}

\section{PROEFSCHRIFT}

ter verkrijging van de graad van doctor aan de Universiteit Maastricht, op gezag van de Rector Magnificus, Prof. mr. M.J. Cohen, volgens het besluit van het College van Decanen, in het openbaar te verdedigen op vrijdag 28 februari 1997 om 12:00 uur

door

Ludovicus Maria Leonardus Anna van Etten

geboren op 3 mei 1958 te Bunde 
Promotor:

Prof. dr. ir. W.H.M. Saris

Co-promotores:

Dr. K.R. Westerterp

Dr. F.T.J. Verstappen

Beoordelingscommissie: Prof. dr. H. Kuipers (voorzitter)

Prof. dr. R.A. Binkhorst, Katholieke Universiteit Nijmegen

Prof. dr. A. Huson, Technische Universiteit Eindhoven

Dr. W. Van Mechelen, Vrije Universiteit Amsterdam

Prof. dr. E.F.M. Wouters

Financial support by the Dr. Ir. Van der Laar Foundation for the publication of this thesis is gratefully acknowledged.

Part of the research presented in this thesis was supported by a research grant from the Netherlands Heart Foundation 
In memory of Miet and Henk 
vi 


\section{CONTENTS}

Chapter 1 General introduction

Chapter 2 Effect of body build on weight-training-induced adaptations in body composition and muscular strength $\ldots \ldots \ldots \ldots \ldots \ldots \ldots \ldots \ldots \ldots \ldots \ldots$

Chapter 3 Effect of weight training on energy expenditure and substrate utilization during sleep

Chapter 4 Daily variation in energy expenditure during weight training versus continuous arm cranking and cycling

Chapter 5 Anthropometrical and physiological correlates of energy expenditure during weight training

Chapter 6 Effect of 18-weeks of weight training on exercise economy

Chapter 7 The energy expenditure-heart rate relationship during weight training . 95

Chapter 8 Effect of an 18-week weight-training program on energy expenditure and physical activity

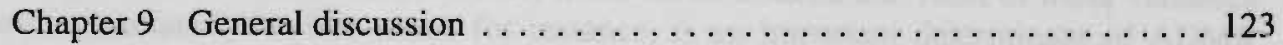

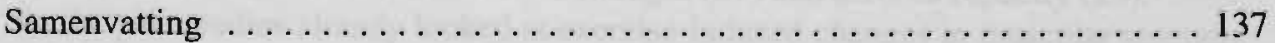

Abbreviations .............................. 141

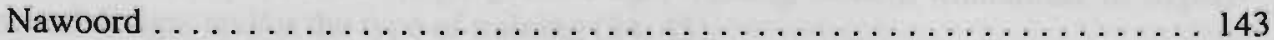

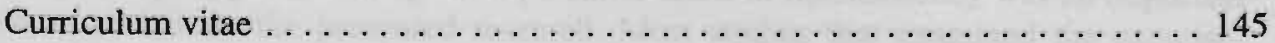

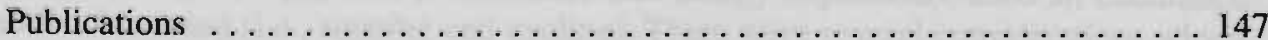




\section{Chapter 1}

\section{General introduction}

Many people participate in physical exercise programs or sport activities. The reason to participate, however, varies widely among people. Depending on interests and physical, social, and/or psychological wellbeing, the consideration to workout (i.e., exercise) will emphasize on social, psycho-emotional, recreational, sporting/athletic, aesthetic/cosmetic or health goals. Although the previous enumeration suggests a clear distinction between initial rationalities, the reason to engage in sports activities as well as the result of physical exercise will hardly ever be confined to only one of the above-mentioned areas.

As a logical consequence of the multiple-usability of exercise, physical exercise is subject to research in various scientific disciplines. All these different fields of research use their discipline-specific methods and parameters. This thesis will concentrate on exercise-induced adaptations in a restricted number of anthropometrical and physiological parameters related to energy expenditure (EE), body composition and physical performance. With regard to energy expenditure the emphasis will lie on an exercise-induced effect on Average Daily Metabolic Rate (ADMR), Sleeping Metabolic Rate (SMR), and EE during exercise (EEex). The effect on body composition will be evaluated using the variables Fat-Free Mass (FFM) and Fat Mass (FM). With regard to physical performance the effect on maximal aerobic capacity and muscular strength will be examined. However, it should be noticed that most of these variables are interrelated. Fat-free mass, for instance, is an important determinant of ADMR $(41,42)$, SMR (39), as well as muscular strength (27) and aerobic capacity (29).

In the past studies already looked at exercise-induced changes in muscular strength, aerobic capacity, ADMR and EE during exercise. However, with contrasting results. It is suggested that this inconsistency for the greater part can be explained by differences in the selected population (e.g., gender, age, training status), limitations in experimental design, and/or the type of training $(34,45)$.

Especially "type of training" as a potential cause of inconsistency was an important reason to initiate the presented research. Most studies that examined the exerciseinduced changes in physical performance and energy expenditure used an endurance mode of training (i.e., running and cycling). There were several considerations why a continuous endurance type of exercise was preferred:

- in contrast to interval modes of activity, performance during endurance exercise is largely determined and/or limited by physiological factors and depends for a smaller part on motor skills and tactics like for instance during gymnastics or team 
(ball)sports. Therefore, improvements in physiological determinants will directly affect performance;

- these modes of exercise can relatively easily be standardized and performed in a laboratory setting using a cycle ergometer or a treadmill;

- due to the continuous character of endurance exercise, assumptions can be made on physiological processes during exercise (e.g., substrate utilization) that makes interpretation and data collection easier;

- the large number of people participating and competing in these types of physical activity implicitly signifies practical relevance.

Besides endurance training, a large number of people participate in modes of exercise that have an intermittent or interval character. Soccer, basketball, aerobic dance and weight training are all examples of physical activities that alternate exercise periods with recovery periods. In recent years weight training became an activity performed by an increasing number of enthusiasts. The effect of weight training on ADMR, body composition and performance is particularly interesting to examine because this type of sport/leisuretime activity underlines exercise-induced health and fitness benefits with a specific emphasis on cosmetic/aesthetic improvement (e.g., body composition, overweight, see Fig. 1.l). In view of this objective, weight-training

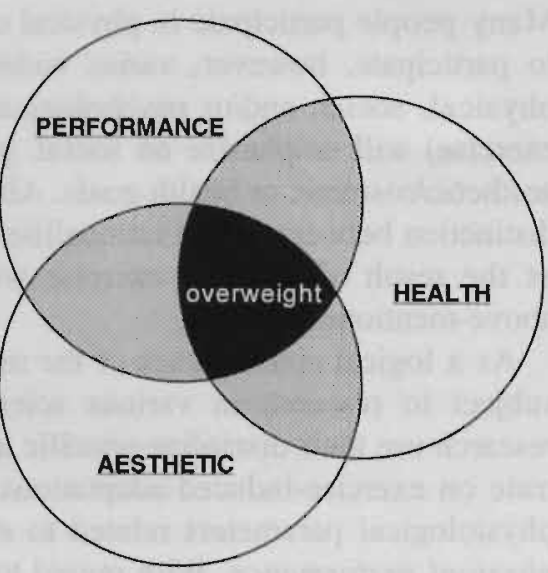

Fig. 1.1. Overweight as the central overlap of the three different areas of interest in weight-training programs.

programs and methods were developed to be suitable for a wide variety of target groups. Accordingly, weight training is a form of training that is reported to be save, even for overweight, hypertensive and cardiac patients, pre-puberal males and the elderly $(10,20,22,49)$. In contrast to other intermittent modes of exercise like game sports, weight-training performance relies for a smaller part on motor control and tactical skills. This makes weight training more suitable for standardization and reproduction in a laboratory setting.

Outlining, this thesis will investigate the effect of weight training within the framework of energy expenditure, body composition and physical performance. Before presenting the studies, some background information is presented on weighttraining exercise, energy expenditure and some methodological considerations.

\section{WEIGHT TRAINING}

Based on the applied overload method or the desired effect, this mode of exercise is also named resistance training or strength training. During a weight-training workout 
relatively short exercise periods are alternated with recovery intervals. Free weights (barbels, dumbbells) or weight-stack machines (constant or variable resistance) are used to increased resistance (i.e., overload) during exercise. Nowadays also hydraulic and pneumatic exercise equipment is available. From the standpoint of muscle contraction, basically three training methods can be distinguished: isotonic, isometric, and isokinetic training:

- Isotonic weight training is the most common method of weight training and involves concentric as well as eccentric contractions. The exercises are performed in sets that consist of a given number of consecutive repetitions (reps). After completing all sets of each exercise in succession, the trainee moves to the next exercise or station. Using the term 'isotonic' to describe this method of weight training could be somewhat confusing because it does not represent a muscular contraction in which the muscle exerts a true constant tension, but rather a contraction against constant external resistance (the tension varies with the mechanical advantage of the joint(s) involved).

- During isometric weight training, contractions should be held for a certain time at a prescribed percentage of maximal contraction. Since this type of training consist of static muscle activity, it is also named static-weight training. This mode of exercise is widely used in rehabilitation programs to counteract strength loss and muscle atrophy.

- Isokinetic weight training is another method often used in rehabilitation. During contraction (mainly concentric) the speed of the movement is mechanically controlled by an isokinetic device. Resistance accommodates the force throughout the entire range of motion.

Table 1.1. Comparative rating of the three types of weight training (from Lamb, 1984).

\begin{tabular}{|c|c|c|c|}
\hline \multirow{2}{*}{ Criterion } & \multicolumn{3}{|c|}{ Comparative rating } \\
\hline & Isotonic & Isometric & Isokinetic \\
\hline Rate of strength gain & Good & Poor & Excellent \\
\hline Rate of endurance gain & Good & Poor & Excellent \\
\hline Strength gain over range of motion & Good & Poor & Excellent \\
\hline Time per training session & Poor & Excellent & Good \\
\hline Expense & Good & Excellent & Poor \\
\hline Ease of performance & Poor & Excellent & Good \\
\hline Ease of progress assessment & Excellent & Good & Poor \\
\hline Adaptability to specific movements patterns & Good & Poor & Excellent \\
\hline Least possibility of muscle soreness & Poor & Good & Excellent \\
\hline Least possibility of injury & Poor & Good & Excellent \\
\hline Skill improvement & Good & Poor & Excellent \\
\hline
\end{tabular}

Table 1.1 summarizes the advantages and disadvantages of the three types of weight training (26). Although isokinetic weight training seems to be superior to the traditional isotonic training method, the somewhat unnatural movement patterns (e.g., constant speed, lack of eccentric contraction) makes this type of training in practice less superior than suggested. Because the high cost of isokinetic equipment, the majority of fitness centres use the traditional free weights and weight stack machines. From this 
practical point of view, the training interventions performed in this thesis used the traditional isotonic type of weight training.

Besides a conventional organisation of weight training (i.e., predetermined number of repetitions and all sets performed in succession before moving to the next exercise), many programs use a circuit type of organisation. The circuit organisation usually has 6-15 stations (exercises) per circuit and the circuit is repeated 2-3 times. At each station a load is selected that fatigues the muscle group in approximately 30 seconds in which as many repetitions as possible should be performed (i.e., maximal number of repetitions and one set per circuit).

Weight-training-induced changes in physical performance. The degree to which weight training affects performance parameters depends on a number of factors including: initial training status, and type, volume, intensity, as well as duration of the weighttraining program. The most obvious weight-training-induced effect on performance is an increase in muscular strength (3). This increase is caused by neural adaptation as well as muscle hypertrophy $(30,35,40)$. Aerobic capacity is another frequently used performance-related effect parameter. With the exception of circuit weight training, resistance training has not generally been regarded as effective in increasing aerobic capacity. However, some studies revealed a small increase in aerobic capacity using a high volume weight-training program. The mechanism by which non-circuit weight training can increase aerobic capacity is still unclear and can only be speculated on. Although it is not likely that weight training enhances muscular determinants of aerobic capacity (e.g., aerobic enzyme activity, mitochondrial numbers and size, fibre composition, muscle capillary density) $(1,9,37,46)$, a change in central factors (i.e., increased cardiac output) could account for the small but significant increase found in some studies (45).

Weight-training-induced changes in body composition and health. As mentioned before, weight training is a sportleisure-time activity that focuses on improving physical performance as well as exereise-induced beneficial effects on health and aesthetic. From cross-sectional as well as longitudinal studies it is known that weight training can positively affect health indicators like resting, exercise, and recovery heart rates; myocardium strength and stroke volume; resting diastolic and systolic blood pressure in hypertensive humans; lipid profiles, glucose tolerance and insulin sensitivity (12, $21,32,38,45,47$ ).

A main health subject of this thesis, however, is to evaluate the effect of weight training on body composition (\%fat). Percentage body fat can be decreased by reduction of fat mass and/or increasing fat-free mass. It is generally accepted that physical exercise exerts this favourable effect on fat mass and fat-free mass (53). An excessive fat mass might be for some people unwanted because of aesthetical reasons. From a health point of view, excess body fat is not favourable because it is associated with an increased risk for cardiovascular disease, diabetes mellitus, high concentrations of total cholesterol and lower HDL-cholesterol, kidney disease and degenerative arthritis (45). The exercise-induced increase in fat-free mass is not only 
favourable because of the concomitant decrease in \%fat. Exercise also reduces the relative decrease in fat-free mass due to dietary restriction $(2,11)$. The latter is an additional beneficial effect because fat-free mass is closely related to both physical performance and resting metabolic rate.

However, results of individual studies showed great variation in exercise-induced changes in body composition. The effect depends on type, intensity and volume of the activity, dietary restriction/control, and population differences (e.g., age, sexe). For instance, exercise-induced changes in fat mass in studies without dietary restrictions ranged from $-1.71 \mathrm{~kg} \cdot$ week $^{-1}$ (17) to no change at all (18). Some investigators suggest that weight training induces a larger increase in fat-free mass and smaller decreases in fat mass compared to endurance training (24). On the other hand, other researchers found comparable changes or even greater decreases in fat mass due to weight training (53). The use of large muscle mass exercises and large training volumes seems to be the key factor in weight-training-induced changes in body composition (45) and therefore the key factor for a beneficial effect on health.

\section{ENERGY EXPENDITURE}

Based on the first law of thermodynamics, excess fat mass can be counteracted by a negative energy balance. A negative energy balance can be realized by increasing energy expenditure and/or decreasing energy intake. The discussed studies are restricted to the increasing energy expenditure (i.e., ADMR) by adding a training program to the regular physical activity regime.

When comparing the effect of an exercise program on ADMR and EE during exercise, the potential effect of training on energy expenditure is somewhat contradictory. On the one hand, the energy cost of the added exercise will increase daily energy expenditure, on the other a higher training status decreases EE during physical activity. This denotes the need to include both phenomenons in the discussion on exercise-induced changes in ADMR.

Average daily metabolic rate (ADMR). ADMR is the sum of the energy required for physical activity, processing food, and to sustain body's vital functions during rest. Currently, the doubly labelled water technique is the most accurate method (1-3\%) to measure ADMR in free living humans (41). This technique is based on the differential elimination of the isotopes ${ }^{2} \mathrm{H}$ and ${ }^{18} \mathrm{O}$ from body water. After a loading dose of both stable isotopes, ${ }^{2} \mathrm{H}$ will be eliminated as water while ${ }^{18} \mathrm{O}$ will be eliminated as water and carbon dioxide. The difference between the two elimination rates is a measure of carbon dioxide production. Isotope enrichment is measured with mass spectrometry in blood, urine or saliva samples. Based on substrate utilization (i.e., estimated by the food quotient (50)), oxygen consumption can be calculated. Subsequently, conventional indirect calorimetry calculations are used to determine energy expenditure.

To identify the range of variation and significant determinants of energy expenditure in free living adults, Schulz (42) compiled 22 studies that used the doubly labelled 
water method. The ADMR ranged from $8.7 \pm 2.2 \mathrm{MJ} \cdot \mathrm{day}^{-1}\left(0.17 \mathrm{MJ} \cdot \mathrm{day}^{-1} \cdot \mathrm{kg}^{-1}\right)$ in females in developing countries $(19,23,43)$ to $29.4 \pm 1.0 \mathrm{MJ} \cdot$ day $^{-1}\left(0.42 \mathrm{MJ} \cdot \mathrm{day}^{-1} \cdot \mathrm{kg}^{-1}\right)$ in a group of four male cyclists engaged in the Tour the France (51). Body mass and fat-free mass revealed to be significant determinants of ADMR. Furthermore, there were large differences in ADMR between ethnic populations. ADMR expressed relative to body mass showed an exceptional low energy expenditure for male as well as female Pima Indians (see Table 1.2).

Table 1.2. Variation in ADMR between different populations and between males and females (from Schulz 1994).

\begin{tabular}{|c|c|c|c|c|}
\hline males & Athletes & $\begin{array}{l}\text { Pima } \\
\text { Indians }\end{array}$ & $\begin{array}{l}\text { Developing } \\
\text { countries }\end{array}$ & Other \\
\hline $\operatorname{ADMR}\left(\mathrm{MJ}^{\mathrm{d}} \mathrm{day}^{-1}\right)$ & $29.4 \pm 1.0$ & $12.0 \pm 2.5$ & $16.2 \pm 4.5$ & $14.2 \pm 2.9$ \\
\hline$A D M R / B M\left(M J \cdot d a y^{3} \cdot \mathrm{kg}^{\prime}\right)$ & 0.42 & 0.14 & 0.28 & 0.17 \\
\hline females & & & & \\
\hline $\operatorname{ADMR}\left(\mathrm{MJ}^{\mathrm{d}}\right.$ day $\left.^{-1}\right)$ & $11.8 \pm 1.3$ & $11.7 \pm 1.9$ & $8.7 \pm 2.2$ & $10.3 \pm 1.9$ \\
\hline ADMR/BM (MJ.day $\left.{ }^{7} \cdot \mathrm{kg}^{\prime}\right)$ & 0.23 & 0.11 & 0.17 & 0.14 \\
\hline
\end{tabular}

Until now only four studies examined the exercise-induced changes in ADMR using the doubly labelled water technique. Three studies revealed an increase in ADMR (4, 5, 31) whereas one study showed no change in ADMR (16). All studies used an endurance type of exercise. No research is performed on the effect of weight training on ADMR.

To obtain more detailed information on ADMR, additional information on the following three separate components of ADMR is collected: the resting metabolic rate (60-75\% of ADMR), the diet-induced thermogenesis (food processing; 10\% ADMR) and the energy cost of physical activity (15-30\% ADMR).

Resting metabolic rate is the energy requirement to sustain the body's vital functions in the wakening state. When measured during sleep the basal energy requirement the term sleeping metabolic rate is used. The difference in energy expenditure between the wakening and sleeping state is approximately 5\% (15). Earlier studies revealed that resting metabolic rate following exercise could be slightly elevated for up to 31 hours (33). Because resting metabolic rate accounts for $60-75 \%$ of daily energy expenditure, relatively small changes can substantially increase energy expenditure over time. With regard to weight training, research on the effect of training on resting metabolic rate is worthwhile because weight training increases fat-free mass being the primary determinant of resting metabolic rate (14).

The physical activity component of ADMR, including the energy cost of the added exercise, is the most variable component of ADMR (39). Large differences in the energy cost of exercise programs are found depending on frequency, volume and intensity of the workouts. Several studies already looked at the energy cost of a single weight-training workout. The relatively low energy cost seems to make this mode of exercise less effective in programs of weight control or weight reduction (28). 
However, previous studies that showed an exercise-induced increase in ADMR revealed that the increase in ADMR largely exceeded the energy cost of the training program itself $(4,5,31)$. Hence, the overall effect of weight training on ADMR could also exceed the energy cost of the workouts.

So, to illuminate and quantify the effect of weight training on ADMR, energy expenditure measurements should also include measurements of the separate components of ADMR.

Energy expenditure during exercise. Especially during prolonged exercise the availability of energy is an important determinant of performance. A person's performance potential, however, is not only restricted by his or her maximal capacity to generate energy, but also the energy cost of submaximal activity. Work reserve (maximal effort minus actual effort) is a measure of the intensity of an activity and therefore a measure of someone's potential to further increase or sustain a certain workload. Work reserve increases as maximal work (i.e., performance) increases or/and if the same work can be performed using less effort. In Fig 1.2. the effect of an increase in maximal work and a decreased relative effort during submaximal performance on work reserve is illustrated by the oxygen uptake during cycling. An improved $\mathrm{VO}_{2}$ max will result in an increased work reserve during cycling at $150 \mathrm{~W}$ (see left panel). If, however, the same work $(150 \mathrm{~W})$ can be performed using less oxygen, work reserve will also increase even without an increase in maximal performance (see middle panel). Since training will most likely affect $\mathrm{VO}_{2} \max$ as well as $\mathrm{VO}_{2}$ submax, training will result in a cumulative increase in work reserve (see right panel).
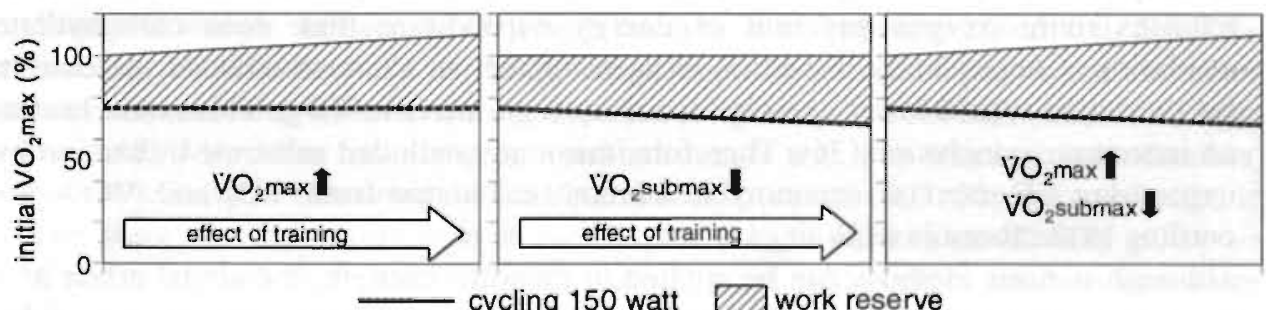

cycling 150 watt

Fig. 1.2. The effect of an increased aerobic capacity (left panel), decreased axygen uptake at 150 watt cycling (middle panel) and a combination of these exercise-induced changes (right panel) on work reserve.

The term "efficiency of movement" is often used to describe EE during exercise. The term "efficiency" suggests exact knowledge about the mechanical work accomplished and the energy expended to do that work. Calculating mechanical work, however, is very difficult if not impossible. Mechanical work does not only consist of the work done on an ergometer or treadmill, but also includes changes in position or resultant velocity of the centre of mass and displacements, velocities and accelerations of body segments. Factors such as transfer of energy, elastic storage and re-use of energy can all change the mechanical energy of a segment and subsequently limit the accuracy of determining mechanical power (52). A comparable problem occurs when determining 
the energy expended to perform the mechanical work. Gross energy expenditure also includes the energy costs of processes not related to the performed mechanical work. Baseline subtractions (e.g., resting metabolic rate, zero workload) are often used to correct for these energy costs. However, baseline values change with increasing work rate and will therefore affect metabolism (e.g., changes in gastrointestinal processes, splanchnic metabolism, body temperature, levels of catecholamines). Depending on the accuracy and method that are used to calculate efficiency, exercise efficiency varies widely even within studies (13). Therefore, baseline subtraction should not be used in calculations of muscle efficiency (44).

Because of the above-mentioned difficulties to obtain accurate estimates of the work done and the energy expended to do that work the term "economy" -defined as the oxygen uptake $\left(\mathrm{VO}_{2}\right.$ submax) required to perform a given task- has become the physiological criterion for "efficiency" of performance (8). Because this thesis focuses on energy expenditure, economy will be expressed in $\mathrm{kJ} \cdot \mathrm{min}^{-1}$ instead of $\mathrm{ml} \mathrm{O}_{2} \cdot \mathrm{min}^{-1}$.

Although energy availability is probably not a limiting factor during weight training, information on exercise economy should be taken into account in the discussion on exercise-induced changes in daily energy expenditure.

\section{METHODOLOGICAL CONSIDERATIONS}

In general, standard routines are used. Sometimes adjustments or concessions are made based on methodological or practical considerations:

- usually exercise economy is expressed as $\mathrm{VO}_{2}$ submax. Fat oxidation, however, requires more oxygen per unit of energy expenditure than does carbohydrate oxidation. Consequently, based on oxygen uptake, an exercise-induced increase in fat oxidation would lower exercise economy (i.e., increase $\mathrm{VO}_{2}$ ). This could lead to an inaccurate conclusion (36). Therefore, this study included substrate utilization by expressing $\mathrm{EE}$ exercise economy in $\mathrm{kJ} \cdot \mathrm{min}^{-1}$ calculated from $\mathrm{VO}_{2}$ and $\mathrm{VCO}_{2}$ according to the formula of Weir (48).

- although various methods can be applied to measure strength, the actual effect of a training program is most accurately measured with a method that closely resembles the training program (i.e., specificity of the strength test) (25). Since an isotonic type of intervention program was performed, an isotonic method of strength measurement would be preferable (e.g., one repetition maximum $=1 \mathrm{RM}=$ largest mass that can be lifted one time). This method, however, is less suitable for subjects with no previous weight-training experience with respect to: coordination, safety, and risk on injury. Furthermore, this method of strength testing is time consuming and needs special facilities (e.g., power racks, especially with respect to leg exercises; weights $>200$ $\mathrm{kg}$ ). Because there was no equipment available in the laboratory to determine 1RM in a safe way, isokinetic strength tests were performed using angular velocities close to the speed of the isotonic movements.

- because muscular strength could not be measured in a safe and accurate way, differentiation in training loads between subjects during the measurement of "EE during 
exercise" was not possible (i.e., comparable intensity between subjects). Therefore, two sets of standard weights were used based on the lowest estimated level of training to ensure that all subjects could perform at least all exercises using the lowest set of weights.

\section{OUTLINE OF THE THESIS}

Three studies (named $\mathrm{A}, \mathrm{B}$, and $\mathrm{C}$ ) were conducted to evaluate the effect of weight training on energy expenditure, body composition and physical performance.

Study-A was a twelve-week weight-training intervention. The first aim of study-A was to examine the influence of differences in body build (fat-free mass relative to fat mass and height) on weight-training-induced changes in body composition and physical performance (chapter 2). Because weight training is known to affect fat-free mass, it was conceivable that weight training could affect sleeping metabolic rate. So, the second aim of study-A was to examine whether weight training would affect sleeping metabolic rate (chapter 3 ).

A next step was to examine the effect of weight training on daily energy expenditure. Before studying the effect of weight training on ADMR, a reliable method had to be found to measure EE during weight training. Therefore, the cross-sectional study-B was conducted to examine whether EE during weight training could be measured accurately. After quantifying day-to-day variability in energy expenditure during weight training (chapter 4 ), the efficacy of on weight-training intervention on ADMR could be evaluated (study-C).

Study-C was an eighteen-week weight-training intervention to examine in detail the effect on ADMR. Because of the potential opposite effect of physical training on energy expenditure, both EE during exercise (chapter 6) and ADMR (chapter 8) were examined. Also data on sleeping metabolic rate and non-training (spontaneous) physical activity as measured with a triaxial accelerometer $(6,7,31)$ were collected to obtain more detailed information on the effect of weight training on ADMR.

Combined data from study-B and study-C were additionally used to determine which anthropometrical and/or physiological variables affect EE during this specific weight-training protocol (chapter 5) and to validate the application of heart rate monitoring in predicting energy cost of weight-training workouts (chapter 7).

Finally, results of the previous studies will be summarized and results from the different studies will be integrated and discussed (chapter 9).

\section{REFERENCES}

1 Adams, G. R., B. M. Hather, K. M. Baldwin, G. A. Dudley. Skeletal muscle myosin heavy chain composition and resistance training. J. Appl. Physiol. 74(2):911-915, 1993. 
2 Ballor, D. L., V. L. Katch, M. D. Becque, C. R. Marks. Resistance weight training during caloric restriction enhances lean body weight maintenance. Am. J. Clin. Nutr. 47:19-25, 1988.

3. Berger, R. Effect of varied weight training programs on strength. Res. Quart. 33(2):168-181, 1962.

4 Bingham, S. A., G. R. Goldberg, W. A. Coward, J. H. Cummings. The effect of exercise and improved physical fitness on basal metabolic rate. Brit. J. Nutr. 61:155-173, 1989.

5 Blaak, E. E., K. R. Westerterp, O. Bar-Or, L. J. M. Wouters, W. H. M. Saris. Total energy expenditure and spontaneous activity in relation to training in obese boys. Am. J. Clin. Nutr. 55:777-782, 1992.

6 Bouten, C. V., K. R. Westerterp, M. Verduin, J. D. Janssen. Assessment of energy expenditure for physical activity using a triaxial accelerometer. Med. Sci. Sports Exerc. 26(12):1516-1523, 1994.

7 Bouten, C. V. C., W. P. H. G. Verboeket-van de Venne, K. R. Westerterp, M. Verduin, J. D. Janssen. Daily physical activity assessment: comparison between movement registration and doubly labeled water. J. Appl. Physiol. 81(2):10191026, 1996.

8 Cavanagh, P. R., R. Kram. The efficiency of human movement-a statement of the problem. Med. Sci. Sports Exerc. 17(3):304-308, 1985.

9 Costill, D. L., J. Daniels, W. Evans, W. Fink, G. S. Krahenbuhl, B. Saltin. Skeletal muscle enzymes and fiber composition in male and female track athletes. J. Appl. Physiol. 40(2):149-154, 1976.

10 Crozier Ghilarducci, L., G. Holly, E. Amsterdam. Effects of high resistance training in coronary artery disease. American Journal of Cardiology 64:866-870, 1989.

11 Dale van, D., P. F. M. Schoffelen, F. Hoor ten, W. H. M. Saris. Effects of addition of exercise to energy restriction on 24-hour energy expenditure, sleeping metabolic rate and daily physical activity. Eur. J. Clin. Nutr. 43:441-451, 1989.

12 Fripp, R. R., J. L. Hodgson. Effect of resistive training on plasma lipid and lipoprotein levels in male adolescents. The Journal of Pediatrics :926-931, 1987.

13 Gaesser, G. A., G. A. Brooks. Muscular efficiency during steady-rate exercise: effects of speed and work rate. J. Appl. Physiol. 38(6):1132-1139, 1975.

14 Garby, L., J. S. Garrow, B. Jorgensen, et al. Relation between energy expenditure and body composition in man: specific energy expenditure in vivo of fat and fatfree tissue. Eur. J. Clin. Nutr. 42:301-305, 1987.

15 Goldberg, G. R., A. M. Prentice, H. L. Davies, P. R. Murgatroyd. Overnight and basal metabolic rates in men and women. Eur. J. Clin. Nutr. 42:137-144, 1988.

16 Goran, M. I., E. T. Poehlman. Endurance training does not enhance total energy expenditure in healthy elderly persons. Am. J. Physiol. 263(26):E950-E957, 1992.

17 Hadjiolova, I., L. Mintcheva, S. Dunev, M. Daleva, S. Handjiev, L. Balabannski. Physical working capacity in obese women after an exercise programme for body weight reduction. Int. J. Obesity 6:405-410, 1982. 
18 Hagan, R. D., S. J. Upton, L. Wong, J. Whittam. The effect of aerobic conditioning and/or caloric restriction in overweight men and women. Med. Sci. Sports Exerc. 18(1):87-94, 1986.

19 Heini, A., Y. Schutz, E. Diaz, A. M. Prentice, R. G. Whitehead, E. Jequier. Freeliving energy expenditure measured by two independent techniques in pregnant and nonpregnant Gambian women. Am. J. Physiol. 261:E9-E17, 1991.

$20 \mathrm{Hill}$, D. W., S. D. Butler. Haemodynamic responses to weightlifting exercise. Sports Med. 12(1):1-7, 1991.

21 Hurley, B. F., J. M. Hagberg, A. P. Goldberg, et al. Resistive training can reduce coronary risk factors without altering VO2max or percent body fat. Med. Sci. Sports Exerc. 20:150-154, 1988.

22 Hurley, B. F., R. A. Redmond, R. E. Pratley, M. S. Treuth, M. A. Rogers, A. P. Goldberg. Effects of strength training on muscle hypertrophy and muscle disruption in older men. Int. J. Sports Med. 16(6):378-384, 1995.

23 Huss-Ashmore, R., J. L. Goodman, T. E. Sibiya, T. P. Stein. energy expenditure of Young Swazi women as measured by the doubly-labelled water method. Eur. J. Clin. Nutr: 43:737-748, 1989.

24 Katch, F. I., S. S. Drumm. Effects of different modes of strength training on body composition and anthropometry. Clin. Sport Med. 5(3):413-459, 1986.

25 Kraemer, W. J., A. C. Fry, Strength testing: development and evaluation of methodology, in Physiological assesment of human fitness, Maud, P. J. and C. Foster, Eds., 1 th ed. Champain, IL: Human Kinetics, 1995, pp. 115-139.

26 Lamb, D. R., Physiology of exercise, 2nd ed. New York: Macmillan, 1984.

27 Maughan, R. J. Relation between muscle strength and muscle cross-sectional area. Implications for training. Sports Med. 1:263-269, 1984.

28 McArdle, W. D., G. F. Foglia. Energy cost and cardiorespiratory stress of isometric and weight training exercises. J. Sports Med. Phys. Fitness 9:23-30, 1969.

29 McArdle, W. D., F. I. Katch, V. L. Katch, Individual differences and measurement of energy capacities, in Exircise physiology: energy, nutrition and human performance, McArdle, W. D., F. I. Katch, and V. L. Katch, Eds., 3th ed. Philadelphia: Lea \& Febiger, 1991.pp. 199-232.

30 Mcdonagh, M. J. N., C. T. M. Davies. Adaptive response of mammalian skeletal muscle to exercise with high loads. Eur. J. Appl. Physiol. 52:139-155, 1984.

31 Meijer, G. A. L., G. M. E. Jansisen, K. R. Westerterp, F. Verhoeven, W. H. M. Saris, F. Hoor ten. The effect of a 5-month endurance-training programme on physical activity: evidence for sex-difference in the metabolic response to exercise. Eur. J. Appl. Physiol. 62:11-17, 1991.

32 Miller, W., W. Sherman, J. Ivy. Effect of strength training on glucose tolerance and post-glucose insulin response. Med. Sci. Sports Exerc. 16:539-543, 1984.

33 Molé, P. Impact of energy intake and excrcisc on resting metabolic rate. Sports Med. 10:72-87, 1990.

34 Morgan, D. W., P. E. Martin, G. S. Krahenbuhl. Factors affecting running economy. Sports Med. 7:310-330, 1989. 
35 Moritani, T., H. A. DeVries. Neural factors versus hypertrophy in the time course of muscle strength gain. Am. J. Phys. Med. 58:115-130, 1979.

36 Pate, R. P., C. A. Macera, S. P. Bailey, W. P. Bartoli, K. E. Powell. Physiological, anthropometric, and training correlates of running economy. Med. Sci. Sports Exerc. 24(10):1128-1133, 1992.

37 Ploutz, L. L., P. R. Tesch, R. L. Biro, G. A. Dudley. Effect of resistance training on muscle use during exercise. J. Appl. Physiol. 76(4):1675-1681, 1994.

38 Poehlman, E. T., A. W. Gardner, P. A. Ades, et al. Resting energy metabolism and cardiovascular disease risk in resistance-trained and aerobically trained males. Metabolism 41(12):1351-1360, 1992.

39 Ravussin, E., C. Bogardus. Relationship of genetics, age, and physical fitness to daily energy expenditure and fuel utilization. Am. J. Clin. Nutr. 49:968-975, 1989.

40 Sale, D. G. Neural adaptation to resistance training. Med. Sci. Sports Exerc. 20(5):S135-S145, 1988.

41 Schoeller, D. A., C. R. Fjeld. Human energy metabolism: what have we learned from the doubly labeled water method? Ann. Rev. Nutr. 11:355-373, 1991.

42 Schul, L. O., D. A. Schoeller. A compilation of total daily energy expenditure and body weights in healthy adults. Am. J. Clin. Nutr. 60:676-681, 1994.

43 Singh, J., A. M. Prentice, E. Diaz, et al. Energy expenditure if Gambian women during peak agricultural activity measured by the doubly-labelled water method. Brit. J. Nutr. 62:315-329, 1989.

44 Stainsby, W. N., B. L. Gladden, J. K. Barclay, B. A. Wilson. Exercise efficiency: validity of baseline subtractions. J. Appl. Physiol. 48(3):518-522, 1980.

45 Stone, M. H., S. J. Fleck, N. Travis Triplet, W. J. Kraemer. Health and performance-related potential of resistance training. Sports Med. 11(4):210-231, 1991 .

46 Tesch, P. A., A. Thorsson, P. Kaiser. Muscle capillary supply and fiber type characteristics in weight and power lifters. J. Appl. Physiol. 56(1):35-38, 1984.

47 Toth, M. T., E. T. Poehlman. Resting metabolic rate and cardiovascular disease risk in resistance- and aerobic-trained middle aged women. Int. J. Obesity 9:691$698,1995$.

48 Weir de, J. B. New methods for calculating metabolic rate with special reference to protein metabolism. J. Physiol. 109:1-9, 1949.

49 Weltman, A., C. Janney, C. B. Rians, et al. The effects of hydraulic resistance strength training in pre-pubertal males. Med. Sci. Sports Exerc. 18(6):629-638, 1986.

50 Westerterp, K. R. Food quotient, respiratory quotient, and energy balance. Am. J. Clin. Nutr. 57 (suppl):759S-765S, 1993.

51 Westerterp, K. R., W. H. M. Saris, F. Hoor ten. Use of the doubly labeled water technique in humans during heavy sustained exercise. J. Appl. Physiol. 61(6):2162-2167, 1986.

52 Williams, K. R. The relationship between mechanical and physiological energy estimates. Med. Sci. Sports Exerc. 17(3):317-325, 1985. 
53 Wilmore, J. H. Variations in physical activity habits and body composition. Int. J. Obesity 19(sup 4):S107-S112, 1995. 
22 


\section{Chapter 2}

\section{Effect of body build on weight-training-induced adaptations in body composition and muscular strength}

Ludo M.L.A. van Etten, Frans T.J. Verstappen, and Klaas R. Westerterp*

Institute of Movement Sciences and ${ }^{\star}$ Department of Human Biology, Maastricht University, Maastricht, The Netherlands

Med. Sci. Sports Exerc. 26(4):515-521, 1994

\section{ABSTRACT}

The aim of the present study was to investigate whether weight-training-induced adaptations in body composition and isokinetic strength differ as a function of body build. Body build of a subject was characterized as the extent to which a person's fat-free mass index $\left(F F M I=\right.$ fat-free mass $\cdot$ height $\left.{ }^{2} ; \mathrm{kg} \cdot \mathrm{m}^{-2}\right)$ differs from the regression of $F F M I$ over fat mass index $\left(F M I=\right.$ fat mass height $\left.{ }^{-2} ; \mathrm{kg} \cdot \mathrm{m}^{-2}\right)$ as derived from a sedentary male population ( $n=77)$. From this population two groups with either a slender $(n=10)$ or a solid ( $n=11$ ) body build were selected. For 12 wh the subjects performed a weighttraining program twice a week. Training induced a significant $(P<0.05)$ increase in fat-free mass (FFM) in the solid group (1.6 kg, 2.3\%) in contrast with the slender group, which showed no significant change in FFM. Both groups showed comparable decreases in fat mass (FM; slender: $-1.7 \mathrm{~kg},-10.8 \%$ versus solid: $-2.4 \mathrm{~kg},-11.3 \%$ ) and increases in strength (on average 13.8\%). In conclusion, the increase in FFM due to a weight-training program is modified by body build. This modification, however, is restricted to a larger increase in the solidly built group. 


\section{INTRODUCTION}

Muscle, fat and bone are the three major structural components that model the human body build. Visual differences in body dimensions among individuals are readily apparent, including differences in height, weight and regional fat distribution (e.g., waisthip ratio). Body composition differences, namely differences in fat-free mass (FFM) and fat mass (FM), may be less apparent. The actual body build of a subject is determined, in part, by genotype and physical activity. Leading a more active lifestyle, including both occupational and leisure time activities, positively increases the FFM/FM ratio $(22,23,26)$. Furthermore, specific types of physical training lead to specific changes in body composition. Weight training mainly increases FFM whereas endurance-type activities mainly decrease FM (10). The relative contribution of genetics and the environment to body build are not well studied or quantified. In healthy individuals, the size of the skeleton and therefore height, can presumed to be mainly genetically determined.

Like body build, the ability to achieve a high level of sports performances is determined, in part, by genetic factors and physical training. Despite quality training. wide variability still exists in physical performance among elite athletes. Thus, coaches and sports medicine scientists are interested in the connection between body build and physical performance. Previous research (11) has reported the biometrical benefits of height, muscle mass (absolute quantity) and proportional body dimensions for a variety of sports. The benefit of using proportional dimensions is the possibility to compare individuals or groups irrespective of differences in height. Proportional dimensions mostly express the size of body parts relative to other bodily dimensions within a person, e.g., body mass index (BMI) which expresses weight relative to height $\left(\mathrm{kg} \cdot \mathrm{m}^{-2}\right)$. Sometimes bodily dimensions are also compared with reference values based on a large number of subjects. The somatogram introduced by Behnke and Wilmore (1) and the somatotype originated by Sheldon and modified by Heath and Carter (8) are examples of methods to describe body build by using proportional bodily dimensions relative to other dimensions as well as reference values.

The influence of body build is relevant to consider when the effect of different training programs, training methods or equipment are compared. The response to the intervention may be biased by differences in initial body build of the experimental population. This can lead to an over- or underestimation of the studied independent variables and thus to false conclusions.

The aim of the present study was to investigate whether weight-training-induced changes in body composition and strength are a function of initial body build. Additionally, attention is paid to a possible relation between changes in bodily dimensions and physical performance. For this study two groups of healthy untrained sedentary males were selected with a contrasting body build described as the heightnormalized quantity of FFM corrected for FM. Subsequently subjects trained two times a week for $12 \mathrm{wk}$. The response was measured by comparing body composition and strength before and after the intervention. 


\section{METHODS}

Subjects. Subjects were healthy clerks recruited from various offices. The recruitment criteria were male sex, age between 25 and $45 \mathrm{yr}$, a sedentary lifestyle and not participating in a regular training program for at least $2 \mathrm{yr}$ prior to the study. Seventy-seven subjects met these criteria and gave their written informed consent. Body composition, FFM and FM, were calculated from skinfold thickness (3) and body mass (see Table 2.1). From this recruited group two experimental groups (slender and solid group) were selected with contrasting body build.

\begin{tabular}{|c|c|c|c|c|}
\hline Variables & $\begin{array}{c}\text { recruited } \\
(n=77)\end{array}$ & $\begin{array}{c}\text { slender } \\
(n=10)\end{array}$ & $\begin{array}{c}\text { solid } \\
(n=11)\end{array}$ & $\mid$ dif SI-So $\mid$ \\
\hline age (year) & $34 \pm 4.8$ & $38 \pm 3.4$ & $34 \pm 4.7$ & 4.00 \\
\hline weight $(\mathrm{kg})$ & $83.9 \pm 11.5$ & $74.1 \pm 7.9$ & $90.2 \pm 9.7$ & $16.1^{* *}$ \\
\hline height (m) & $1.79 \pm 0.07$ & $1.80 \pm 0.04$ & $1.77 \pm 0.06$ & 0.03 \\
\hline $\mathrm{BMI}\left(\mathrm{kg} \cdot \mathrm{m}^{-2}\right)$ & $26.1 \pm 3.2$ & $22.6 \pm 3.7$ & $28.8 \pm 2.1$ & $6.2^{* \star}$ \\
\hline fat $(\%)$ & $24.0 \pm 5.6$ §ी & $24.2 \pm 4.8$ ๆ & $23.0 \pm 4.2$ ? & 1.2 \\
\hline FFM $(\mathrm{kg})$ & $63.4 \pm 7.0$ & $55.9 \pm 4.1$ & $69.2 \pm 5.7$ & $13.3^{* *}$ \\
\hline$F M(k g)$ & $20.5 \pm 6.5$ & $18.2 \pm 5.1$ & $21.0 \pm 5.5$ & 2.8 \\
\hline FFMI (kg FFM.m.' & $19.7 \pm 1.7$ & $17.1 \pm 1.2$ & $22.1 \pm 1.0$ & $5.0^{* *}$ \\
\hline FMI $\left(\mathrm{kg} \mathrm{FM} \cdot \mathrm{m}^{-2}\right)$ & $6.4 \pm 2.1$ & $5.5 \pm 1.4$ & $6.7 \pm 1.6$ & 1.2 \\
\hline $\begin{array}{l}\text { FFMI-FFMI } \\
(\mathrm{kg} \text { prodicted } \\
\end{array}$ & $0 \pm 1.5$ & $-2.3 \pm 1.2$ & $2.3 \pm 1.0$ & $4.6^{\star \star}$ \\
\hline
\end{tabular}

Values are means $\pm S D$ (level of significance: $" P<0.05, " P<0.01$ ).

Body composition was measured using skinfold thickness (\$) or hydrostatic weighing ( $\|)$.

Body build. The description of body build was based on a two-compartment model of body composition in which body weight is divided into FFM and FM. The active component FFM was used to describe body build. To allow comparisons between individuals, FFM was corrected for height and FM. FFM and FM relative to height were in analogy with the body mass index (BMI) expressed as fat-free mass index $\left(\mathrm{FFMI}=\mathrm{FFM} \cdot\right.$ height $\left.^{-2} ; \mathrm{kg} \mathrm{FFM} \cdot \mathrm{m}^{-2}\right)$ and fat mass index $\left(\mathrm{FMI}=\mathrm{FM} \cdot \mathrm{height}^{-2} ; \mathrm{kg} \mathrm{FM} \cdot \mathrm{m}^{-2}\right.$ ) $(24,26)$. In addition, BMI is equal to the sum of FFMI and FMI. To quantify the relationship between FFMI and FMI (6), FFMI was plotted as a function of FMI (sce Fig. 2.1). The regression equation expresses the quantitative relationship between FFMI and FMI (FFMI predicted $=17.566+0.335 \times \mathrm{FMI} ; \mathrm{r}=0.409 ; \mathrm{P}<0.001$ ). Individual body build was characterized on an interval scale by subtracting $F F M I_{\text {predicted }}$ from the actual FFMI. The $60 \%$ prediction interval of FFMI given FMI (see Fig. 2.I) was used to ensure a clear contrast in body build. After verifying body composition with the hydrostatic weighing technique, subjects beyond this prediction interval were assigned 
to either the slender group ( $\mathrm{n}=10$; actual FFMI smaller than FFMI $I_{\text {predicted }}$ ) or solid group $(\mathrm{n}=11$; actual FFMI greater than FFMI predicted $)$. Three subjects were excluded because of a FMI not within two standard deviations of the mean. For interpretation of the applied concept, examples of body build are given in Figure 2.2 .

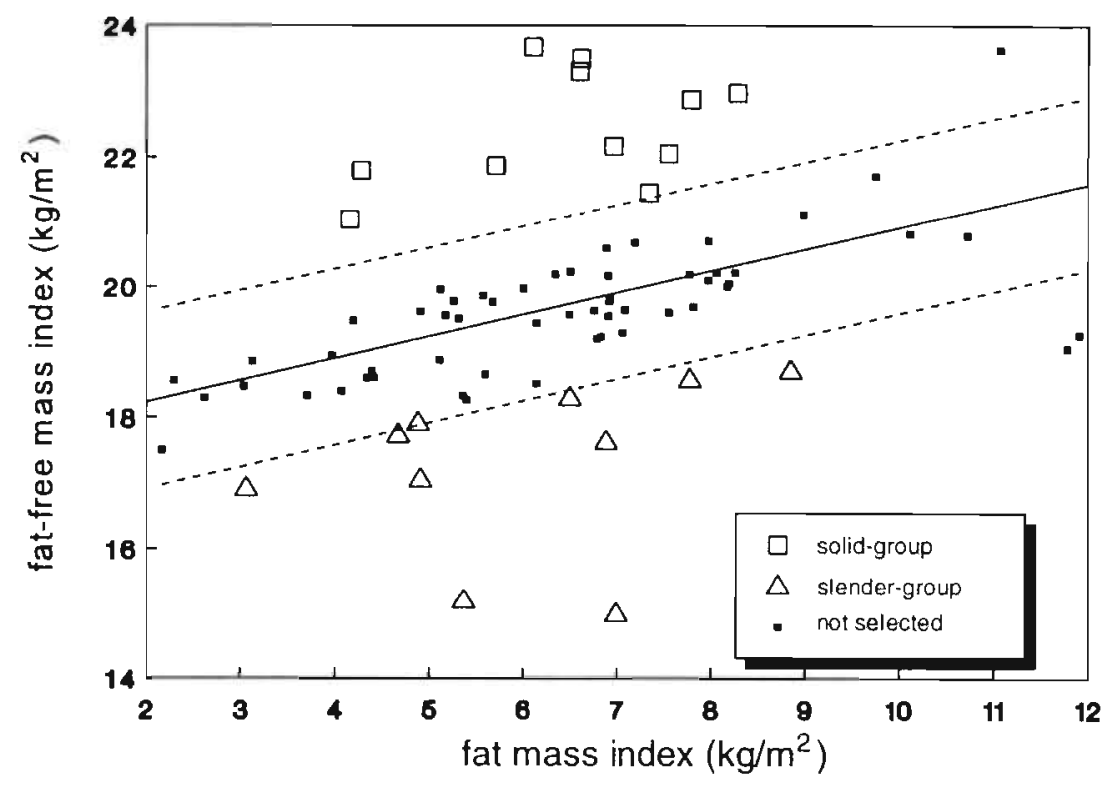

Fig 2.1. Fat-free mass index plotted as a function of fat mass index of the recruited population ( $n=77$;

$\left.F F M I_{\text {sedered }}=17.566+0.335 \times F M I ; r=0.409 ; P<0.001\right)$. The dotted lines are the borderlines of the $60 \%$ prediction interval of FFMI given FMI. Subjects beyond this interval were assigned to either the slender group $(n=10)$ or solid group ( $n=11)$.

Body composition. After the selection procedure all measurements concerning body composition were taken the morning after a controlled overnight stay at the laboratory where the subjects abstained from eating and drinking for $14 \mathrm{~h}$. Body mass was measured after emptying the bladder to an accuracy of $\pm 5 \mathrm{~g}$ on an electronic scale (Mettler, E1200). Body density was measured using the hydrostatic weighing technique with simultaneous measurement of residual lung volume with the helium dilution technique (Volugraph 200, Mijnhardt). Water density was corrected for temperature. Percent fat was calculated from body density assuming a FFM and FM density of 1.097 and $0.901 \mathrm{~kg} \cdot \mathrm{l}^{-1}$, respectively. This resulted in the following equation for percentage of fat:

$$
F A T(\%)=\left(\frac{504.28}{\text { body density }}-459.69\right)
$$




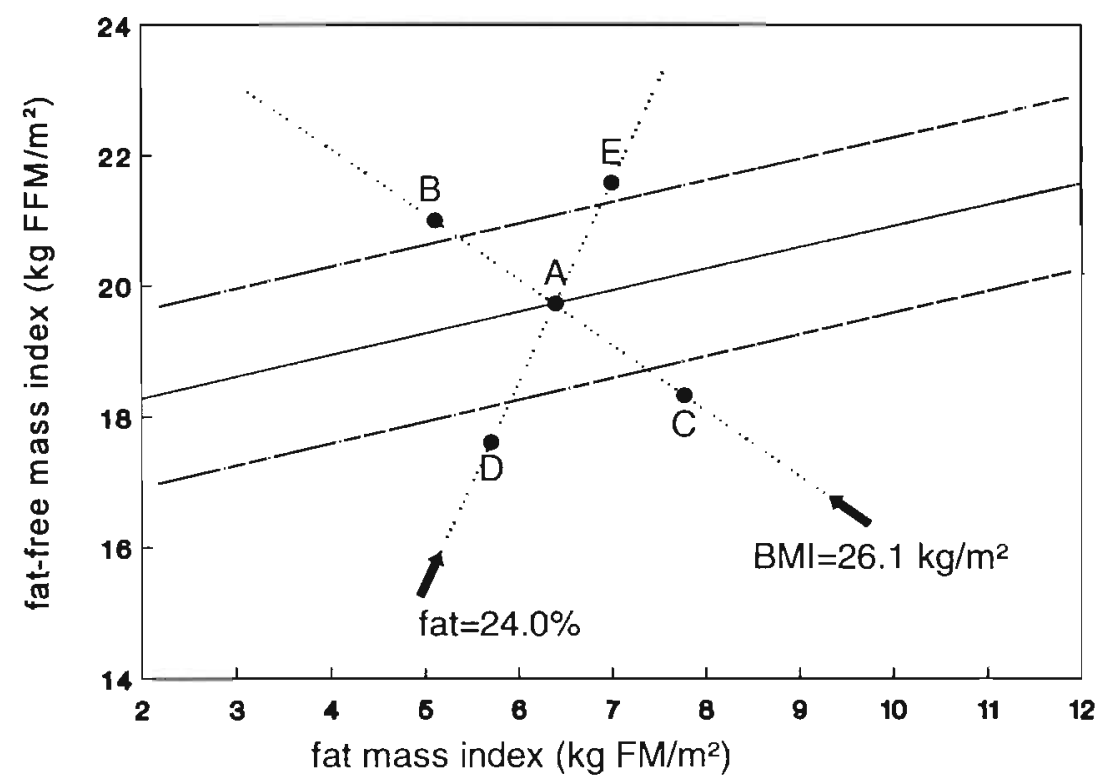

Fig. 2.2. The effect of changes in weight, height or \%fat on the body build of subject-A representing the mean anthropometric characteristics of the recruited group. Changes are printed in italics.

$\begin{array}{lllll}\text { subject } & \text { weight } & \text { height } & \text { (BMI) } & \text { \%flat } \\ \text { A } & 83.8 & 1.793 & (26.1) & 24.0 \\ \text { B } & 83.8 & 1.793 & (26.1) & 19.5 \\ \text { C } & 83.8 & 1.793 & (26.1) & 29.8 \\ \text { D } & 74.9 & 1.793 & (23.3) & 24.0 \\ \text { E } & 91.6 & 1.793 & (28.5) & 24.0 \\ \text { D } & 83.8 & 1.896 & (23.3) & 24.0 \\ \text { E } & 83.8 & 1.714 & (28.5) & 24.0\end{array}$

Muscular strength. Muscular strength in the upper thigh and shoulder/arm was measured using an isokinetic dynamometer (Cybex II) at several angular velocities: knee flexion and extension at $1.047,3.142$ and $4.189 \mathrm{rad} \cdot \mathrm{s}^{-1}(60,180)$ and $240^{\circ} \cdot \mathrm{s}^{-1}$, respectively); shoulder/arm during a bench press movement at 1.047 and $2.094 \mathrm{rad} \cdot \mathrm{s}^{-1}$ (60 and $120^{\circ} \cdot \mathrm{s}^{-1}$, respectively). Subjects performed one set of five repetitions at each velocity from which the peak torque was noted as parameter for maximal strength.

Training program. The subjects trained independently, twice a week, on nonconsecutive days for $12 \mathrm{wk}$. using an individually prescribed training program based on the subject's weight and baseline performance at the respective exercises. The program consisted of $10 \mathrm{~min}$ warming up (cycling at 100-150 W), 14 strength exercises using free weights and machine gym equipment (see Table 2.2) and 5 min of cooling down (cycling at 100-150 W) completed with stretching exercises. In weeks 2 and 3 the initial single set of 15 repetitions (reps) per exercise was doubled and tripled, respectively, without changing the training weight. Throughout weeks 4,5 and 6 , 
training weight was adjusted as strength levels increased provided that the subjects were able to perform three sets of 15 reps. Thus, during the first six weeks the nature of the training program changed from familiarizing and skill development to more serious resistance training. From week 7 the design of the training program was furthermore adjusted for seven exercises (marked with a diamond $(\checkmark)$ in Table 2.2). The one repetition maximum (1RM) of the exercise was measured to adjust the training weights to $65-70 \%$ of 1 RM (14). The number of repetitions per set was changed from 15 to 10 repetitions in the first two sets and an all out effort in the third set (18). This all out effort was used as criterion to further adjustment of the training weight: less than seven reps the training weight was lowered and more than 13 reps the training weight was increased with one unit of weight $(2.5,5$ or $10 \mathrm{~kg}$. depending on the apparatus and muscle group used). In case of the unmarked exercises (see Table 2.2) the adjustment procedure of the first 6 wk was continued. The training program was regularly supervised by a fitness instructor to guide and encourage the subjects and to control the use of correct techniques.

Aerobic power. Maximal aerobic power was measured before and after the training period with a progressive cycling test to consider the specificity of the training.

Table 2.2. Training protocol from weeks 1-12. Subjects trained independently, two times a week on nonconsecutive days.

\begin{tabular}{lccccc}
\hline Exerclse & week & week & week & week 4-6 & week 7-12 \\
& 1 & 2 & 3 & & \\
\hline
\end{tabular}

Flys

Seated Lats Pulley

- Leg Press

- Butterlly

- Triceps Pushdown

Sit Ups

Calf Raises

Leg Curl

- Chest Press

- Leg Extension

- Over Head Lats Pulley

- Shoulder Raises

Preacher Bench Curl

Leg Raises
3 sets of 15 reps + load increment if possible

2 sets of 10 reps + rep third set maximal s if reps $>13$ then load increment

3 sets of max reps

3 sets of 15 reps + load increment if possible

$\begin{array}{cccc}1 \text { set of } & 2 \text { sets } & 3 \text { sets } & \begin{array}{c}3 \text { sets of } 15 \\ \text { reps + load }\end{array} \\ 15 \text { reps } & \text { of } 15 & \text { of } 15 & \text { increment if } \\ \text { reps } & \text { reps } & \text { possible }\end{array}$

2 sets of 10 reps + third set maximal reps if reps $>13$ then load increment

3 sets of 15 reps + load increment if possible 
Statistical analysis. Unpaired and paired $t$-tests were used to analyze the differences between the groups and changes within a group, respectively. When comparing more than two population means, analysis of variance and the Student-Newman-Keuls post-hoc test was used. A probability value of $\mathrm{P}<0.05$ was considered significant.

\section{RESULTS}

Physical characteristics. In Table 2.3 the mean changes $(\Delta)$ in body composition are presented for: all subjects $(\mathrm{n}=21$ ), the slender group ( $\mathrm{Sl} ; \mathrm{n}=10$ ), the solid group (So; $\mathrm{n}=11)$ and the absolute differences between the slender and solid group ( $\mid$ dif Sl-So|). Changes (\%) relative to initial values are also presented because of initial differences in body composition and physical performance between the groups.

Table 2.3. Changes in weight, fat-free mass (FFM), fat mass (FM) and body build after 12 wk of weight training.

\begin{tabular}{|c|c|c|c|c|}
\hline & $\begin{array}{c}\text { all subjects } \\
(n=21)\end{array}$ & $\begin{array}{c}\text { slender } \\
(n=10)\end{array}$ & $\begin{array}{l}\text { solid } \\
(n=11)\end{array}$ & |dif SI-So| \\
\hline \multicolumn{5}{|c|}{ absolute changes } \\
\hline$\Delta$ weight (kg) & $-1.1 \pm 2.1^{*}$ & $-1.5 \pm 1.7^{\star}$ & $-0.8 \pm 2.5$ & 0.7 \\
\hline$\triangle F F M(k g)$ & $0.9 \pm 1.3^{* *}$ & $0.3 \pm 0.9$ & $1.6 \pm 1.4^{* *}$ & $1.3^{*}$ \\
\hline$\Delta F M(k g)$ & $-2.1 \pm 1.7^{* *}$ & $-1.7 \pm 1.4^{* *}$ & $-2.4 \pm 1.9^{* *}$ & 0.7 \\
\hline \multicolumn{5}{|c|}{ changes relative to pretraining values } \\
\hline$\Delta$ weight $(\%)$ & $-1.3 \pm 2.5^{*}$ & $-1.9 \pm 2.3^{*}$ & $-0.9 \pm 2.6$ & 1.0 \\
\hline$\triangle \mathrm{FFM}(\%)$ & $1.5 \pm 2.0^{\star *}$ & $0.5 \pm 1.7$ & $2.3 \pm 2.0^{* *}$ & $1.8^{*}$ \\
\hline$\triangle F M(\%)$ & $-10.9 \pm 8.4^{* *}$ & $-10.5 \pm 8.4 * *$ & $-11.3 \pm 8.8^{* *}$ & 0.8 \\
\hline $\begin{array}{l}\Delta \text { body build } \\
\left(\mathrm{kg} F F M \cdot \mathrm{m}^{2}\right)\end{array}$ & $0.52 \pm 0.5^{* *}$ & $0.26 \pm 0.3^{*}$ & $0.75 \pm 0.5^{* \star}$ & $0.49^{* *}$ \\
\hline
\end{tabular}

Values are means $\pm S D$ (level of signilicance: " $P<0.05, " * P<0.01$ ).

The entire group showed a significant change in body weight $(-1.1 \pm 2.1 \mathrm{~kg},-1.3$ $\pm 2.5 \%)$, FFM $(0.9 \pm 1.3 \mathrm{~kg}, 1.5 \pm 2.0 \%)$ and FM $(-2.1 \pm 1.7 \mathrm{~kg},-10.9 \pm 8.4 \%)$. An additional way to compare the changes in body composition is a comparison of the changes in body build. This method combines changes in FMI and FFMI and hence changes in FM and FFM since height remains the same. The entire group became a more solid body build after $12 \mathrm{wk}$ of weight training. Regarding the subgroups, most of the within group changes were also significant except the changes in weight within the solid group and the changes in FFM within the slender group. Comparing the size of the changes between both subgroups, there is a significantly larger increase in FFM for the solid group $(1.6 \pm 1.4 \mathrm{~kg}$ vs $0.3 \pm 0.9 \mathrm{~kg}$ and $2.3 \pm 2.0 \%$ vs $0.5 \pm 1.7 \% ; \mathrm{P}<0.05)$. The solid group also showed a significantly larger change in body build $(0.75 \pm 0.45$ $\mathrm{kg} / \mathrm{m}^{2}$ versus $0.26 \pm 0.30 \mathrm{~kg} / \mathrm{m}^{2} ; \mathrm{P}<0.05$ ). 


\section{Inittal strength}

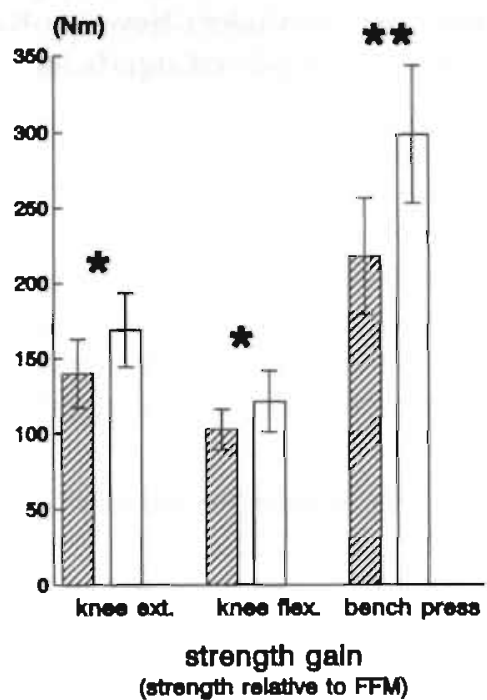

8trength galn

(absoluto)
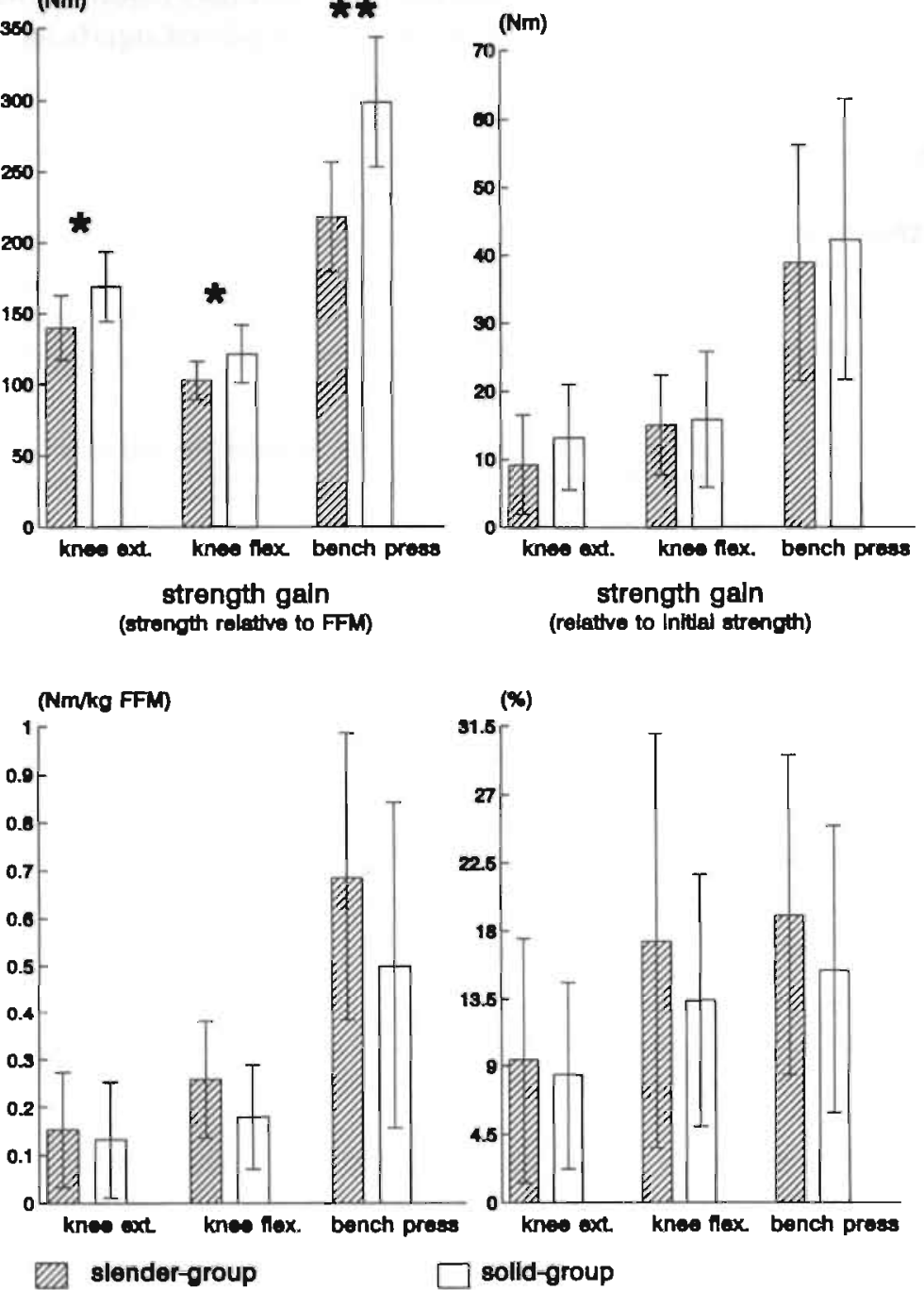

Fig. 2.3. Initial data and changes (mean $\pm S D$ ) in strength at the three movements tested. The initial data differed between both group ( $P<0.05, " P<0.01$ ). Strength was significantly increased $(P<0.001)$ in both subgroups but did not significantly differ between the slender-and solid group. The knee extensor muscles showed a smaller strength gain than the knee flexor and arm/shoulder muscles $(P<0.01)$.

Muscular strength. Before the training period, strength (maximal torque) differed significantly between the groups (see Fig. 2.3), whoreas strength relative to FFM $\left(\mathrm{Nm} \cdot \mathrm{kg} \mathrm{FFM}{ }^{-1}\right)$ was similar at all movements tested. Strength, absolute as well as relative to FFM, significantly increased in both subgroups at all angular velocities and 
movements tested (sce Fig. 2.3). No significant difference in $\Delta$ strength between both subgroups has been found. The relative (\%) increase in strength in the knee extensor is significantly lower than the increase in the knee flexor and shoulder/arm muscles $(8.8$ $\pm 7.0 \%$ vs $15.3 \pm 11.2 \%$ and $17.1 \pm 9.9 \%$ ). There was no significant correlation between initial strength and absolute $\Delta$ strength. Despite a highly significant correlation between initial FFM and the parameters of strength $(r=0.73,0.59$ and 0.85 for knee extension, knee flexion and shoulder/arm muscles, respectively: $\mathrm{P}<0.001$ ), there was no significant correlation between $\triangle F F M$ and $\Delta$ strength.

Aerobic power. Maximal aerobic power was not influenced by weight training.

Training program. Training compliance was similar in the slender and the solid group (97.1 $\pm 4.4 \%$ and $92.4 \pm 7.4 \%$, respectively). The mean absolute training load during the last 6 wk of the training program differed significantly between the slender and solid group ( $43.0 \pm 7.4 \mathrm{~kg}$ vs $51.3 \pm 9.5 \mathrm{~kg}$; $\mathrm{P}<0.05$, respectively). However, training load relative to FFM was comparable $\left(0.76 \pm 0.1 \mathrm{~kg} \cdot \mathrm{kg} \mathrm{FFM}^{-1}\right.$ versus $0.73 \pm 0.1 \mathrm{~kg} \cdot \mathrm{kg}$ $F_{F M}^{-1}$ ). There was no significant correlation between mean absolute training load and $\triangle F F M$ or $\triangle$ strength (absolute and relative to pre-training values).

\section{DISCUSSION}

The aim of the present study was to investigate the effect of body build on weighttraining-induced adaptations in body composition and strength. The physical adaptation in two groups with different body build were compared. The solid group showed a significant absolute and relative increase in FFM $(P<0.05)$. The slender group showed no significant change in FFM. Both groups showed comparable decreases in FM and increases in strength and no change in maximal aerobic power.

Physical characteristics. The mean increase in FFM for the entire group $(+0.9 \mathrm{~kg})$ is in agreement with findings of other weight-training studies. A review of the literature did not provide any information on the effect of initial dillerences in FFM on the training response. The few studies that did include initial differences in FFM in their experimental design were primarily focused on differences between sexes. These studies revealed no influence of the initial amount of FFM. In general, men had a larger absolute increase in FFM and strength but percentage increases were similar in both sexes $(2,9)$. The use of different sexes, however, makes these studies less appropriate as data for comparison.

The mean decrease in FM for the entire group $(-2.1 \mathrm{~kg})$ was somewhat larger then usually found in comparable weight-training studies (on average $1 \mathrm{~kg}$ ). It cannot be excluded that the training-induced changes in FM and/or FFM were partly due to changes of energy intake $(7,15)$. However, the poor reliability of methods to measure dietary intake accurately (27) made it little meaningful to record eventual changes in energy intake in the present study. The similar $\triangle F M$ in both groups suggests that an 
eventual change in dietary intake could only have had a minor contribution to the measured difference in $\triangle$ FFM between the groups. Furthermore, it is not likely that a change of eating habits would only occur in one of the groups.

Body weight decreased significantly in the slender group as a consequence of a larger discrepancy between the increase in FFM and a decrease in FM $(-1.5 \pm 1.7 \mathrm{~kg})$ compared with the solid group $(-0.8 \pm 2.5 \mathrm{~kg})$. The large standard deviation indicates the interindividual variation in $\Delta$ weight. These data demonstrate the limited validity of $\Delta$ weight as a parameter to evaluate dietary or training interventions since it masks adaptation in FFM and FM.

Muscular strength. At the beginning of the program the solid group was stronger than the slender group. The initial amount of FFM correlated significantly with strength $(\mathrm{P}=0.001)$. This is in agreement with other studies showing that strength is primarily determined by the quantity of FFM and the physiological cross-section of the muscle $(13,19,28)$. In spite of this relationship, no significant correlation was found between the increase in FFM and the increase in strength. Surprisingly, the slender group had a comparable increase in strength without a change in FFM. This discrepancy between $\Delta$ strength and $\triangle F F M$ may partly be attributed to the process of neural adaptation to training (20). In the beginning of a training program neural adaptation, if compared with muscular adaptation (hypertrophy), accounts for the larger proportion of the strength increment $(14,16)$. The contribution of muscle hypertrophy increases slowly during the training period and after 3-6 wk becomes the dominant factor. The magnitude of the contribution of neural factors depends on the familiarity with the performed movement or test protocol. The difference in strength gain between the everyday knee extension movement $(8.8 \pm 7.0 \%)$ and the atypical knee flexion (15.3 $\pm 11.2 \%)$ and bench press $(17.1 \pm 9.9 \%)$ movement is in concordance with this latter finding. Another factor that might partly explain the discrepancy found between $\triangle F F M$ and $\Delta$ strength is the specificity of the strength tests. Each test measures $\Delta$ strength in the muscle groups involved in contrast with the increase in FFM which was presumably distributed all over the body. The interindividual variation in the contribution of neural adaptation and $\triangle F F M$ to the increase in strength might explain the lack of a significant correlation between $\triangle F F M$ and $\Delta$ strength.

Body build. This study applied a nearly unknown method to describe body build. The reason for not using a more commonly used method to classify humans by their physical shape lies in the fact that these methods use skinfolds and girths $(1,8)$. These descriptions mostly fit the visual perception but do not make a clear distinction between FFM and FM. Furthermore, studies revealed the limited accuracy of girth to estimate the total amount of or changes in FFM (28). The reliability of the present method to describe body build is restricted to the precision in the determination of body composition and the measurement of height. The application of the heightnormalized index FFMI appeared to be an appropriate method to eliminate differences in height (correlation FFM and height: $r=0.65, \mathrm{P}<0.001$; correlation FFMI and height: $\mathrm{r}=-0.1, \mathrm{P}=0.19 ; \mathrm{n}=77$ ). Further, the quantitative relationship in this study between 
FFM and FM was in agreement with findings of other studies. Dietary intervention studies revealed that FFM contributes for a quarter to a third to changes in body weight (5). The classification of body build by the extent to which a person's FFMI differs from the regression of FFMI over FMI seems a workable method to distinguish between individuals with different height-normalized quantities of FFM and FM. The difference in FFM between the slender and solid group was $13.3 \mathrm{~kg}$ FFM. However, this method is only usable if a valid reference regression equation of FFMI over FMI is available.

This study used several exclusion criteria in combination with a specific definition of body build to eliminate well-known determinants of a training response like age $(12,17,21)$, sex (25), absolute quantity of fat mass (6) and the initial level of training (21). The training stimulus as determinant differed, with respect to the mean training load, between the groups. This was mainly the consequence of the intergroup difference in FFM. The mean absolute training load, however, was not significantly correlated with $\triangle F F M$. Therefore, it seems plausible that the difference in $\triangle F F M$ between both groups is due to the initial difference in body build. The data of the present study, however, do not explain the underlying mechanism responsible for this difference in $\triangle F F M$. A genetic determined potential to increase FFM seems a possible explanation which also could explain the initial differences in body build. Some investigators reported a preferential hypertrophy of type II fibers (4) in weight-trained subjects, suggesting a fiber-type composition modified adaptation to weight training. Another possible explanation lies not so much in the capacity to produce FFM, which refers to the final amount of FFM, but in a difference in adaptation speeds. This hypothesis can be tested by prolonged weight-training studies with several intermediate measurements.

In conclusion, the data of the present study indicate that body build, defined as the initial quantity of fat-free mass corrected for fat mass and height, modifies the weighttraining-induced changes in FFM. After 12 weeks of weight training, individuals with a solid body build increased their FFM whereas slenderly built individuals did not show a significant change in FFM. The data confirm earlier studies that weight training is an appropriate method to increase muscular strength and to decrease FM. Additional research over a longer period is nceded to confirm and extend these findings and to study the mechanism responsible for differences in weight-traininginduced changes in FFM. Reference values for various populations (e.g., males/ females, trained/sedentary or age groups) based on large numbers of subjects should be determined to enable other investigator to utilize and implement FFMI and FMI in describing their study subjects. 


\section{REFERENCES}

1 Behnke, A. R., J. H. Wilmore, Application of the various field methods, in Evaluation and regulation of Body Build and Composition. Englewoods Cliffs, NJ: Prentice-Hall, 1974, pp. 75-81.

2 Cureton, K. J., M. A. Collins, D. W. Hill, F. M. Mcelhannon. Muscle hypertrophy in men and women. Med. Sci. Sports Exerc. 20(4):338-344, 1988.

3 Durnin, J. V. G. A., J. Womersley. Body fat assessed from total body density and its estimation from skinfold thickness: measurement on 481 men and women aged from 16 to 72 ye. Brit. J. Nutr. 32:77-97, 1974.

4 Faulner, J. A., T. P. White, Adaptations of skeletal muscle to physical activity, in Exercise, Fitness and Health: a Consensus of Current Knowledge, Bouchard, C., R. J. Shephard, T. Stephens, J. R. Sutton, and B. D. McPherson, Eds. Champaign, IL: Human Kinetics, 1990, pp. 265-279.

5 Forbes, G. B., The companionship of lean and fat: some lessons from body composition studies, in New techniques in Nutritional Research, Whitehead, R. G. and A. Prentice, Eds. San Diego: Academic press, 1991, pp. 317-329.

6 Forbes, G. B. Exercise and body composition. J. Appl. Physiol. 70:994-997, 1991.

7 Forbes, G. J. Body composition as effected by physical activity and nutrition. Federation Proc. 44:343-347, 1985.

8 Heath, B. H., J. E. L. Carter. A modified somatotype method. Am. J. Phys. Anthrop. 27:57-74, 1967.

9 Holloway, J. B., T. R. Baechle. Strength training for female athletes. A review of selected effects. Sports Med. 9:216-228, 1990.

10 Katch, F. I., S. S. Drumm. Effects of different modes of strength training on body composition and anthropometry. Clin. Sport Med. 5(3):413-459, 1986.

II Katch, F. I., V. L. Katch. The body composition profile. Techniques of measurement and applications. Clin. Sports Med. 3:31-63, 1984.

12 Larson, L. Physical training effects on muscle morphology in sedentary males at different age. Med. Sci. Sports Exerc. 14(3):203-206. 1982.

13 Maughan, R. J. Relation between muscle strength and muscle cross-sectional area. Implications for training. Sports Med. 1:263-269, 1984.

14 Mcdonagh, M. J. N., C. T. M. Davies. Adaptive response of mammalian skeletal muscle to exercise with high loads. Eur. J. Appl. Physiol. 52:139-155, 1984.

15 Meredith, C. N., W. R. Frontera, K. P. O'Reilly, W. J. Evans. Body composition in elderly men: Effect of dietary modification during strength training. J. Am. Geratr. Soc. 40:155-162, 1992.

16 Moritani, T., H. A. DeVries. Neural factors versus hypertrophy in the time course of muscle strength gain. Am. J. Phys. Mid. 58:115-130, 1979.

17 Pollock, M. L., G. A. Dawson, H. S. Miller, et al. Physiologic responses of men 49 to 65 years of age to endurance training. J. Am. Geriatr. Soc. 24:97-104, 1976.

18 Reid, C. M., R. A. Yeater, I. H. Ullrich. Weight training and strength, cardiorespiratory functioning and body compositon of men. Brit.J. of Sports Med. 21:40$44,1987$. 
19 Ryushi, T., K. Hakkinen, H. Kauhanen, P. V. Komi. Muscle fiber characteristics, muscle cross-sectional area and force production in strength athletes, physical active males and females. Scan. J. Sports Sci. 10:7-15, 1988.

20 Sale. D. G. Neural adaptation to resistance training. Med. Sci. Sports Exerc. 20(5):S135-S145, 1988.

21 Saltin, B., L. H. Hartley, A. Kilbom, I. Astrand. Physical training in sedentary middle-aged and older men. II oxygen uptake, heart rate, and blood lactate concentration at submaximal and ma. Scan. J. Clin. Lab. Invest. 24:323-334, 1969.

22 Slattery, M. L., D. R. Jacobs. The inter-relation of physical activity, physical fitness, and body measurements. Med. Sci. Sports Exerc. 19(6):564-569, 1987.

23 Tremblay, A., J. P. Despres, C. LeBlanc, et al. Effect of intensity of physical activity on body fatness and fat distribution. Am. J. Clin. Nutr. 51:153-157, 1990.

24 VanItallie, B. T., M. U. Yang, S. B. Heymsfield, R. C. Funk, R. A. Boileau. Height-normalized indices of the body's fat-free mass and fat mass: potentially usefull indicators of nutritional status. Am. J. Clin. Nutr. 52:953-959, 1990.

25 Wells, C. L., Women, Sport and Performance: A Physiological Perspective, . Champaign, IL: Human Kinetics, 1985, pp. 239-240.

26 Westerterp, K. R., G. A. L. Meijer, A. D. M. Kester, L. Wouters, F. Hoor ten. Fatfree mass as a function of fat mass and habitual activity level. Int. J. Sports Med. 13:163-166, 1992.

27 Westerterp. K. R., W. P. H. G. Verboeket-van de Venne, G. A. L. Meijer, F. Hoor ten, Self-reported intake as a measure for energy intake. a validation against doubly labelled water, in Obesity in Europe 91, Ailhaud. (3., B. Guy-Grand, M. Lafontan, and D. Ricquier, Eds. London: John Libbey \& Company, 1992, pp. 17 22.

28 Young, A., M. Stokes, J. M. Round, R. H. T. Edwards. The effect of high-resistance training on the strength and cross-sectional area of the human quadriceps. Eur.J.of Clinical Investigat. 13:41 1-417, 1983. 


\section{Chapter 3}

\section{Effect of weight training on energy expenditure and substrate utilization during sleep}

Ludo M.L.A. van Etten, Klaas R. Westerterp and Frans T.J. Verstappen Institute of Movement Sciences, Department of Human Biology and Department of Movement Sciences, University Maastricht, Maastricht, The Netherlands Med. Sci. Sports Exerc. 27(2):188-193, 1995

\section{ABSTRACT}

This study was performed to investigate the effect of weight training (12 weeks; 21 male subjects) on energy expenditure and substrate utilization during sleep. Sleeping metabolic rate (SMR) as measured in a respiration chamber was calculated according to three procedures: the lowest mean energy expenditure of a shiftable three hours interval between 0:00 and 6:00 (SMR3) and SMR over predetermined intervals from 3:00 to 6:00 (SMR3-6) and from 0:00 to 6:00 (SMR0-6). In analogy with SMR the corresponding respiratory quotients were expressed as RER3, RER3-6 and RER0.6. Changes in body composition were assessed from changes in body weight, body volume (densitometry) and total body water (deuterium dilution). Weight training induced an increase in fat-free mass $(+1.1 \pm 1.3 \mathrm{~kg} ; P<0.001)$ and a decrease in fat mass $(-2.3 \pm 1.5 \mathrm{~kg} ; P<0.001)$ and body woight $(-1.1 \pm 2.1 \mathrm{~kg} ; P<0.05)$. There was no significant change in SMR, irrespective of the way SMR was expressed. Only RER3 decreased significantly (from $0.82 \pm 0.04$ to $0.79 \pm 0.02 ; P<0.05$ ). Remarkably RER3, RER3-6 and RER0.6 were highly negative correlated with the pre-training RER $(r=-0.93,-0.91$ and -0.90 , respectively: $P<0.001)$ resulting in a diminished variation in post-training $R E R(P<0.001)$. These results suggest that weight training has no effect on SMR but increases relative fat utilization in low fat oxidizers and vice versa for individuals displaying high pre-training lipid oxidation. 


\section{INTRODUCTION}

In the Western world overweight is not only unwanted for health reasons but also from a cosmetic point of view. Considering the interest in weight-control programs, a large group of people struggle with overweight. An excess quantity of fat is the result of a disturbed balance between energy intake (EI) and energy expenditure (EE). In the past decades a lot of research has been done on the effect of dietary- (i.e., EI) and/or training- (i.e., EE) interventions.

The most obvious solution against a positive energy balance is a reduction of EI by changing the diet. The findings of such studies showed a substantial decrease in fat mass (FM) but also an unwanted decrease in fat-free mass (FFM) and EE. By examining the three separate components of EE (Thermic Effect of Activity: TEA $\approx 15-$ $30 \%$ of EE. Thermic Effect of Feeding: TEF $\approx 10 \%$ and Resting Metabolic Rate: RMR $\approx 60-75 \%$ ). this decrease in EE was mainly due to a decrease in the RMR-component (17) and could for the greater part be explained by a decrease in FFM $(10,13)$. Subsequently, studies were designed to prevent this decrease in FFM and its concomitant decrease in RMR by adding a physical training program to a diet intervention. These studies failed to conclusively demonstrate a reduced decrease in RMR but nevertheless recommended a combination of diet and training because of the smaller cuntribution of FFM in total weight loss and the beneficial effect on physical fitness $(4,5,8,11,12,21)$.

The interest in physical exercise as a tool to regulate energy balance increased when longitudinal as well as cross-scctional studies revealed that an increase in physicil activity was not restricted to the TEA-component of EE. Excess post-exercise oxygen consumption (EPOC), TEF and RMR appeared to increase (15). In particular the effect of training on RMR was of interest because a small change in RMR could lead to a substantial change in daily EE. The findings of the latter studies were inconsistent with respect to the effect of physical training and trained state on RMR. This inconsistency can mainly be explained by technical and methodological differences as well as interindividual differences in genetic-related factors $(17,18)$. So, further research is necessary to elucidate training-induced adaptations in EE.

Up to now most of the studies used an endurance-type of training usually in combination with a diet to realize a training-induced adaptation in $\mathrm{EE}$. In recent years weight training (i.e., resistance training) has become more and more accepted by the large public as an appropriate method to workout. Weight-training-induced adaptations differ from endurance-training-induced adaptations by a larger increase in FFM (9). This larger increase in FFM and the lact that FFM is the primary determinant of RMR (7) make it worthwhile to study the effect of weight training on RMR. Thereby, little is known about the effect of weight training in combination with an ad libitum diet (3).

The aim of the prescint study was to investigate the effect of weight training on RMR and substrate utilization during sleep and the relationship with changes in body composition. Subjects $(n=21)$ followed a weight-training program over a period of twelve weeks. RMR and substrate utilization were measured during an overnight stay 
in a respiration chamber and therefore the term sleeping metabolic rate (SMR) is used instead of RMR. Data on body composition and SMR were collected in the beginning and at the end of the training period.

\section{METHODS}

Subjects. Subjects were 21 healthy male clerks, age between 25 and 45 year, not participating in a regular training program for at least two years prior to the study. The subjects trained twice a week, on nonconsecutive days for twelve weeks.

Table 3.1. Training protocol from week 1 to 12. Subjects trained independently, two times a week on non consecutive days.

\begin{tabular}{lccccc}
\hline Exercise & week & week 2 & week 3 & week 4-6 & week 7-12 \\
1 & & & & \\
\hline
\end{tabular}

Fly's

Seated Lats Pulley

3 sets of 15 reps + load increment if possible

- Leg Press

- Buttertly

- Triceps Pushdown

Sit Ups

1 set

2 sets

3 sets

3 sets of 15

3 sets of max reps

Calf Raises

of 15

of 15

of 15

reps + load

increment if possible

2 sets of 10 reps + third set maximal reps

if reps $>13$ then load increment

Leg Curl reps reps reps

- Chest Press

- Leg Extension

- Over Head Lats Pulley

- Shoulder Raises

Preacher Bench Curl

3 sets of 15 reps + load increment if possible

Leg Raises
2 sets of 10 reps + third set maximal reps if reps $>13$ then load increment

3 sets of 15 reps + load increment if possible

reps=repetitions

Training program. The program consisted of ten minutes warming up (cycling), fourteen strength exercises using free weights and machine gym equipment (see Table 3.I) and five minutes of cooling down (cycling) completed with stretching exercises (total duration approximately $1.5 \mathrm{~h}$ ). Initial training load was based on the subject's weight and baseline performance at the respective exercises. From week seven the design of the training program was adjusted for seven exercises (marked with a diamond ( ) in Table 3.I). The training sessions were supervised by a fitness instructor to guide and encourage the subjects and to oversee the use of correct techniques. More detailed 
information on the training program is described elsewhere (22). All subjects provided written informed consent prior to data collection.

In week 2 and week 13 data on body composition and SMR were collected. To reduce confounding by an acute effect of exercise, initial measurements were postponed for one week. All measurements concerning body composition were taken the morning following the overnight stay at the laboratory after voiding the bladder.

Physical characteristics. Body Weight (BW) was measured to an accuracy of $\pm 5 \mathrm{~g}$ on an electronic scale (Mettler, E1200). Body Volume (BV) was measured using the hydrostatic weighing technique with simultaneous measurement of residual lung volume with the helium dilution technique (Volugraph 200, Mijnhardt). Water density was corrected for temperature. Total Body Water (TBW) was mcasured using an isotopic dilution technique. After taking a baseline urine sample, a deuterium $\left({ }^{2} \mathrm{H}\right)$ dilution in a dose to reach an excess of $100 \mathrm{ppm}$ was orally taken directly before bedtime (around 22:30 h). The next moming the second voiding after awaking was collected to determine TBW. The ${ }^{2} \mathrm{H}$ concentration was measured with an Aqua Sira mass spectrometer from VG Isogas Ltd. (Middlewich, England). Because of the exchange of ${ }^{2} \mathrm{H}$ with labile hydrogen of nonaqueous body constituents the total amount of body water is not equal to the ${ }^{2} \mathrm{H}$-dilution space (20). Therefore,

$$
\text { Total Body Water }=\frac{{ }^{2} \mathrm{H} \text {-dilution space }}{1.04}
$$

Initial FFM was calculated from total body water assuming a hydration factor of 0.73 . With the available data on changes in body weight $(\triangle B W)$, body volume $(\triangle B V)$ and $\mathrm{TBW}(\triangle \mathrm{TBW})$, it was possible to use the three-compartment model of Murgatroyd and Coward (14) to determine changes in body composition. The following equations were used to calculate changes in protein mass (PM), FM and FFM while assuming constant mineral mass and a constant proportion of protein, water and mineral in lean tissue:

$$
\begin{aligned}
& \triangle P M=3.046 \triangle B W-0.2852 \triangle T B W-2.741 \triangle B V \\
& \triangle F M=2.741 \triangle B V-0.7148 \triangle T B W-2.046 \triangle B W \\
& \triangle F F M=\triangle T B W+\triangle P M
\end{aligned}
$$

Sleeping metabolic rate. Energy expenditure during sleep was measured during an overnight stay in a respiration chamber (18:00-8:00 h). Dinner was eaten before entering the chamber and subjects were not allowed to eat until body composition was determined the next morning. To minimize the residual effects of training on energy expenditure, subjects were asked to refrain from any exercise on the day of the measurement. Hence, SMR measurement was always $>8$ hours after the last meal and $>30$ hours after the last training session. More technical information on the respiration chamber is described elsewhere (4). Originally sleeping metabolic rate was defined as the lowest mean energy expenditure of a shiftable three hours interval between 0:00 
and 6:00 am (SMR3). In the literature, however, several methods are used to define resting/sleeping metabolic rate. So, in addition energy expenditure during sleep was also calculated according to two other procedures using predetermined intervals:

- SMR3-6: as the mean energy expenditure between 3:00 and 6:00 am;

- SMR0-6: as the mean energy expenditure between 0:00 and 6:00 am.

Substrate utilization. The Respiratory Exchange Ratio (RER) was assumed to reflect the substrate being metabolized. In analogy with SMR, the corresponding respiratory quotient was expressed as RER3, RER3-6 and RER0-6.

Statistical analysis. The effect of exercise training was assessed by using Student's $t$-tests. Regression techniques were used to compare SMR independent of FFM. A probability value of $\mathrm{P}<0.05$ was considered significant.

\section{RESULTS}

Changes in physical characteristics. After twelve weeks of weight training, subjects had lost $1.1 \pm 2.1 \mathrm{~kg}$ body weight $(\mathrm{P}<0.05$ ). Changes in body composition (see Table $3.2)$ consisted of a decrease in FM $(2.3 \pm 1.5 \mathrm{~kg} ; \mathrm{P}<0.001)$ and body volume $(1.4 \pm 2.2$ $1 ; \mathrm{P}<0.01)$ and an increase in TBW $(0.9 \pm 1.01$; $\mathrm{P}<0.001)$ and $\mathrm{PM}(0.3 \pm 0.6 \mathrm{~kg}$; $\mathrm{P}<0.05$ ). The increase in TBW and PM cumulatively resulting in an increase in FFM $(1.1 \pm 1.3 \mathrm{~kg} ; \mathrm{P}<0.001)$.

\begin{tabular}{|c|c|}
\hline $\begin{array}{l}\text { pre-training Body Weight }(\mathrm{kg}) \\
\triangle \mathrm{BW}(\mathrm{kg})\end{array}$ & $\begin{array}{l}82.5 \pm 12.0 \\
-1.1 \pm 2.1^{*}\end{array}$ \\
\hline $\begin{array}{l}\text { pre-training Body Volume (I) } \\
\triangle B V(I)\end{array}$ & $\begin{array}{l}79.1 \pm 11.8 \\
-1.4 \pm 2.2^{*}\end{array}$ \\
\hline $\begin{array}{l}\text { pre-training TBW (I) } \\
\triangle \mathrm{TBW}(\mathrm{I})\end{array}$ & $\begin{array}{l}45.0 \pm 5.4 \\
+0.9 \pm 1.0^{\star *}\end{array}$ \\
\hline $\begin{array}{l}\text { pre-training FFM (kg) } \\
\triangle F F M(k g)\end{array}$ & $\begin{array}{l}61.7 \pm 7.4 \\
+1.1 \pm 1.3^{* *}\end{array}$ \\
\hline $\begin{array}{l}\text { pre-training } F M(\mathrm{~kg}) \\
\triangle F M(\mathrm{~kg})\end{array}$ & $\begin{array}{l}20.9 \pm 5.5 \\
-2.3 \pm 1.5^{* *}\end{array}$ \\
\hline$\triangle$ Protein Mass $(\mathrm{kg})$ & $+0.3 \pm 0.6^{*}$ \\
\hline
\end{tabular}

Sleeping metabolic rate. When analyzing data on SMR one subject had to be excluded because of an attack of fever during his post-training stay in the respiration chamber $\left(\triangle S M R 3+1.1 \mathrm{~kJ} \cdot \mathrm{min}^{-1}\right.$, i.e., $\left.+22.8 \%\right)$. The data on energy expenditure showed no significant weight-training-induced change in SMR3 $\left(0,07 \pm 0.28 \mathrm{~kJ} \cdot \mathrm{min}^{-1} ; \mathrm{P}=0.31\right)$ nor 
in SMR3 per kilogram FFM $\left(0.00 \pm 0.004 \mathrm{~kJ} \cdot \mathrm{min}^{-1} \cdot \mathrm{kg}^{-1} ; \mathrm{P}=0.95\right.$, see Table 3.3). The same held for SMR3-6 and SMR0-6. Additionally the pre- and post-training regression equations of SMR3 given FFM were compared and did not differ (see Fig. 3.1).

Table 3.3. Pre-and post-training values and changes in Sleeping Metabolic Rate (SMR) and Respiratory Exchange Ratio (RER) during sleep

\begin{tabular}{|c|c|c|c|c|c|c|}
\hline \multirow[b]{3}{*}{ SMR3 } & \multicolumn{2}{|c|}{ pre-training } & \multicolumn{2}{|c|}{ post-training } & \multicolumn{2}{|c|}{ difference } \\
\hline & \multicolumn{6}{|c|}{$\left(k J \cdot \min ^{-1}\right)$} \\
\hline & 5.0 & \pm 0.6 & 5.1 & \pm 0.6 & +0.07 & \pm 0.28 \\
\hline SMR3.6 & 5.2 & \pm 0.5 & 5.2 & \pm 0.6 & +0.03 & \pm 0.37 \\
\hline SMR0-6 & 5.2 & \pm 0.7 & 5.2 & \pm 0.6 & +0.02 & \pm 0.32 \\
\hline \multicolumn{7}{|c|}{$\left(\mathrm{kJ} \cdot \mathrm{min}^{-1} \cdot \mathrm{kg}^{-1}\right)$} \\
\hline SMR3/FFM & 0.080 & \pm 0.006 & 0.079 & \pm 0.006 & -0.000 & \pm 0.004 \\
\hline SMR3-6/FFM & 0.082 & \pm 0.006 & 0.081 & \pm 0.007 & -0.001 & \pm 0.006 \\
\hline SMRo-6/FFM & 0.083 & \pm 0.006 & 0.082 & \pm 0.007 & -0.001 & \pm 0.005 \\
\hline $\mathrm{RER}_{3}$ & 0.82 & \pm 0.04 & 0.79 & \pm 0.02 & -0.02 & $\pm 0.04^{*}$ \\
\hline RER3-6 & 0.80 & \pm 0.04 & 0.79 & \pm 0.02 & -0.01 & \pm 0.04 \\
\hline RER $0-6$ & 0.80 & \pm 0.03 & 0.78 & \pm 0.02 & -0.01 & \pm 0.03 \\
\hline
\end{tabular}

Values are means $\pm S D(n=20 ; * P<0.05)$

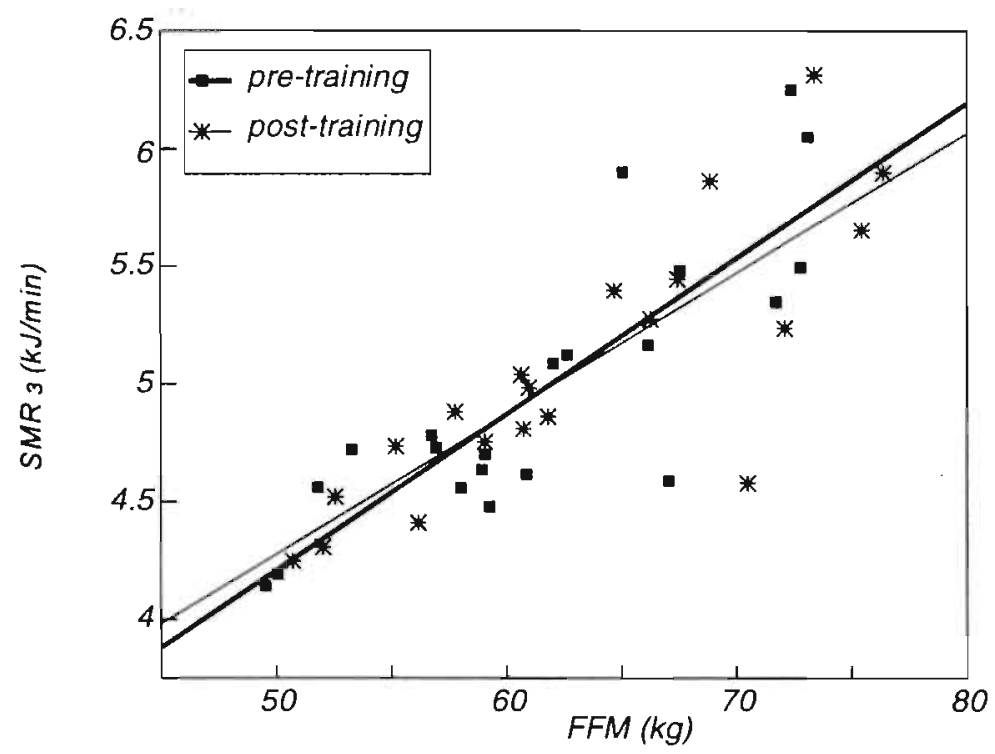

Fig. 3.1. Relationship between fat-free mass (FFM) and sleeping metabolic rate (SMR3) before and after twelve weeks of weight training.

equation [SE] pre- $: 0.907[0.618]+0.066[0.010] \times F F M ; r=0.84$

post- $: 1.303[0.591]+0.060[0.009] \times F F M ; r=0.83$ 
Substrate utilization. The decrease in RER3 $(0.02 \pm 0.04 ; \mathrm{P}=0.035)$ indicated an increased lipid oxidation during sleep. However, RER3-6 and RER0-6 did not significantly change. On the other hand, changes in RER3, RER3-6 and RER0-6 were negative correlated with the pre-training RER ( $r=-0.93,-0.91$ and -0.90 , respectively; $\mathrm{P}<0.001$, see Fig. 3.2) and the post-training standard deviations of RER3, RER3-6 and

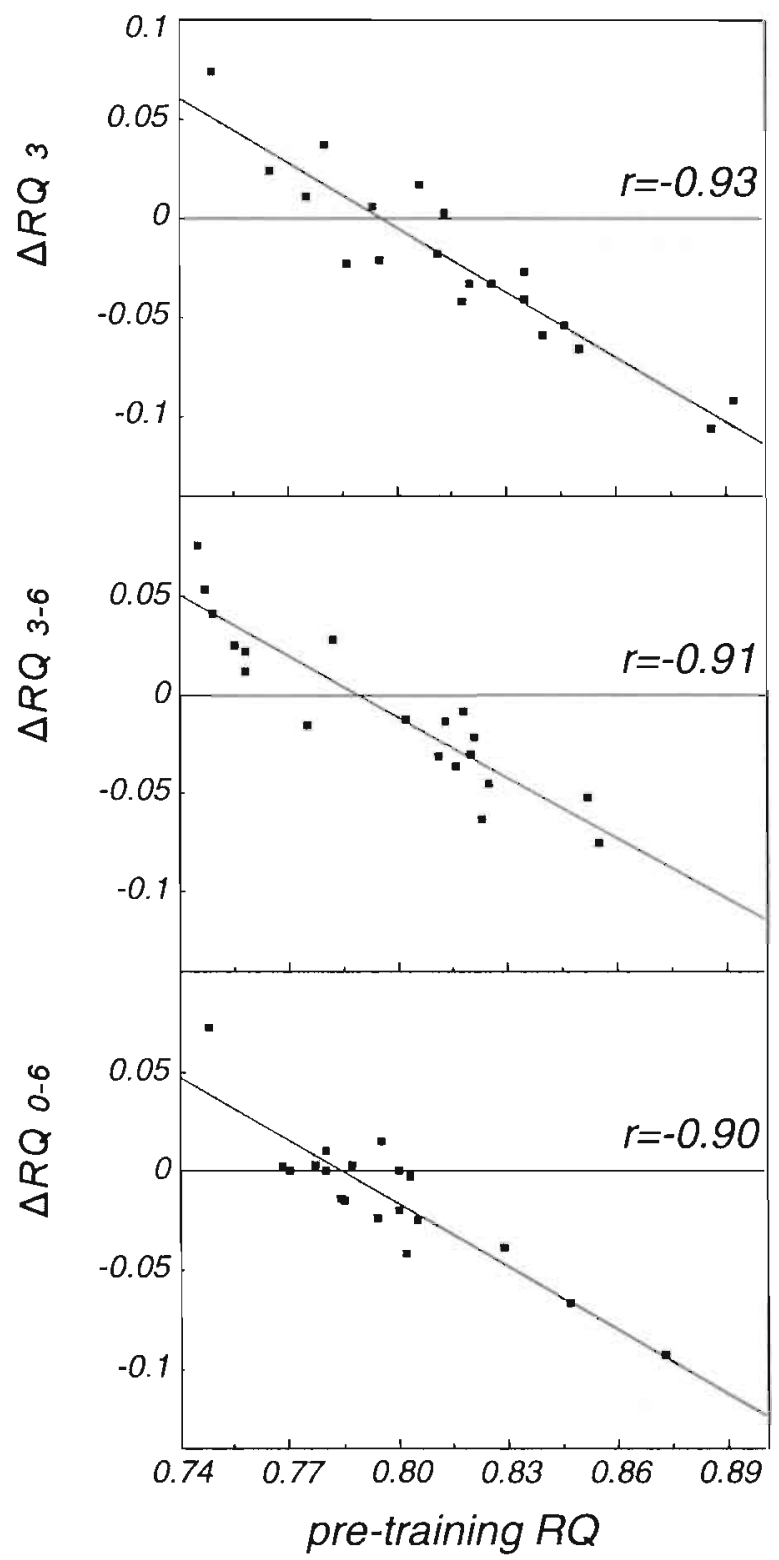

Fig. 3.2. Relationship between $\triangle R E R 3, \triangle R E R 3-6, \triangle R E R 0-6$ and the corresponding pretraining RER $(P<0.001)$. 
RER0-6 were all significantly smaller than the pre-training standard deviation $(0.04$ and 0.03 versus $\pm 0.02 ; \mathrm{P}<0.01$ ). None of the changes regarding body composition were correlated with either $\triangle \mathrm{SMR}, \triangle \mathrm{SMR} / \mathrm{FFM}$ or $\triangle \mathrm{RER}$.

\section{DISCUSSION}

The aim of the present study was to investigate the effects of weight training on energy expenditure and substrate utilization during sleep and their possible relationship with changes in body composition.

Changes in physical characteristics. The weight-training-induced changes in body composition were comparable to findings of other studies albeit the mean decrease in FM $(2.3 \mathrm{~kg})$ was somewhat larger than usually found $(\approx 1 \mathrm{~kg})$. On average the composition of $\triangle \mathrm{FFM}$ (TBW=77\%. $\mathrm{PM}=23 \%$ ) appeared to be identical to the composition of skeletal muscle tissue that contains $77 \%$ water (6). However, individual values showed large differences in the composition of $\triangle F F M$ (regression equation $\triangle \mathrm{PM}$ over $\triangle \mathrm{TBW}: 0.09+0.199 \times \Delta \mathrm{TBW} ; \mathrm{P}=0.14, \mathrm{SE}=0.57$ ), probably due to the limited accuracy of the measurements.

Sleeping metabolic rate. The overnight stay in the respiration chamber offered the opportunity to calculate SMR over several intervals. The only limitation was the respiration chamber protocol that prescribed a continues measuring period of at least three hours to ensure a reliability of $0.5 \pm 2 \%$. Three definitions for SMR were used to examine whether methodological differences in defining SMR could explain the inconsistency in the literature on training interventions and their effect on SMR. Because of the shiftable interval, SMR3 was the best estimation of the lowest energy expenditure during rest. Measurements of RMR (e.g., ventilated hood), however, mostly use a predetermined interval. Compared with a predetermined interval, a shiftable interval logically results in a lower mean energy expenditure (e.g., SMR3<SMR3-6, P<0.001). Therefore SMR3-6 was calculated as a comparable measurement to RMR. Additionally, EE over a prolonged period (six hours; SMR0-6) was calculated as well.

Weight training induced significant changes in FFM $(+1.1 \mathrm{~kg})$ whereas FFM was an important determinant of SMR. So, the data on EE were also expressed relative to FFM to elucidate whether changes in SMR were due to changes in metabolic size or to an altered metabolic rate of FFM. First, SMR was expressed per kilogram FFM (SMR. $\mathrm{kg}^{-1}$ FFM). Since the $y$-intercept of the regression equation of SMR over FFM is not equal to zero the latter method may bias the results (19). So second, also pre- and post-training regression equations were compared. Irrespective of the method that was used to define SMR, there was no significant difference between the absolute or relative pre- and post-training EE during sleep.

Some investigators suggested that the training intensity must reach a minimal threshold of the $\mathrm{VO}_{2} \max$ to affect $\operatorname{SMR}(13,15)$. Because this study used weight 
training instead of endurance training it is of little relevance to express the average training intensity in percentage of aerobic power. It is not likely that the absence of a weight-training-induced adaptation in SMR can be explained by an insufficient training load given the duration of 1.5 hour per session and a maximal load per exercise. Therefore, we conclude that twelve weeks of weight training had no effect on energy expenditure during sleep. This finding is in concordance with a comparable study of Broeder et al. (3) on weight training and energy metabolism in nondieting subjects. The results also agree with those of a cross-sectional study of Bosselaers et al. (2) comparing energy expenditure during sleep corrected for differences in FFM, between body builders and control subjects. However, a cross-sectional study of Poehlman et al. (16) revealed higher resting energy requirements independent of FFM in body builders compared with untrained subjects. The most outstanding contrast with the other studies was the high level of training of the body builders in the latter study (trained 5-6 times. $\mathrm{wk}^{-1}$; extremely low $8 \%$ body fat).

Substrate utilization. The RER3 as a measure for substrate utilization during sleep showed a significant decrease indicating a relatively larger lipid oxidation. Assuming a substrate contribution to SMR from protein of 10-20\%, this mean change in RER3 represents a $\pm 7 \%$ absolute increase in energetic contribution from fat. There is some evidence that this training-induced increase in lipid oxidation is attributed to increased catecholamine levels $(1,21)$. Although this change in substrate utilization is statistically significant, other data did not confirm this finding. RER3-6 and RER0-6 did not differ whereas individual RER-values showed a change in fat contribution ranging from $-24 \%$ to $+35 \%$. This was in agreement with the cross-sectional study of Bosselaers et al. (2) where no significant differences in RER during sleep (0:00-8:30) were found between body builders and control subjects.

It can not be excluded that changes in RER are due to a corresponding change in food composition (23). Unfortunately there are no data available on food composition. The only attention being paid to the subjects diet was an emphatic request not to change their eating habits during the training period.

Although $\triangle R E R$ represents a change in the relative contribution of lipid oxidation to SMR, there was no relationship between $\triangle F M$ and $\triangle R E R$ or $\triangle S M R$. However, a remarkable finding was that $\triangle \mathrm{RER}$ was reversely correlated with pre-training $\mathrm{RER}$ $(\mathrm{P}<0.001)$ which resulted in a diminished variation in post-training $R E R(P<0.01)$. Subjects with a low pre-training RER increased their RER whereas subjects with a high pre-training RER showed a decrease. The change in RER on both sides of the $\mathrm{x}$-intercept (see Fig. 3.2) makes it less plausible that $\triangle R E R$ was a consequence of a change in eating habits. Furthermore, the high correlation between pre-training RER and $\triangle R E R(P<0.001)$ rejects that $\triangle R E R$ was a consequence of regression to the mean. This high correlation with the initial RER in combination with a diminished posttraining variation suggests that weight training moderated substrate utilization during sleep towards a RER ratio of $0.78-0.79$. A plausible mechanism can not be given on the basis of the available data. 
In conclusion, the data of the present study suggest that weight training increases FFM and decreases FM but has no effect on the energy expenditure during sleep. Substrate utilization only changes significantly when expressed as RER3. Conversely, the change in RER is modified by pre-training RER, irrespective of the interval over which the RER was calculated. Furthermore, there is no relationship between changes in body composition and changes in SMR or RER.

\section{REFERENCES}

1 Bielinski, R., Y. Schut, E. Jéquir. Energy metabolism during the postexercise recovery in man. Am. J. Clin. Nutr. 42:69-82, 1985.

2 Bosselaers, I., B. Buemann, O. J. Victor, A. A. Astrup. Twenty-four-hour energy expenditure and substrate utilization in body builders. Am. J. Clin. Nutr. 59:10-12, 1994.

3 Broeder, C. E., K. A. Burrhus, L. S. Svanevik, J. Wilmore. The effects of either high-intensity resistance or endurance training on resting metabolic rate. Am. J. Clin. Nutr. 55:802-810, 1992.

4 Dale van, D., P. F. M. Schoffelen, F. Hoor ten, W. H. M. Saris. Effects of addition of exercise to cnergy restriction on 24-hour energy expenditure, sleeping metabolic rate and daily physical activity. Eur. J. Clin. Nutr. 43:441-451, 1989.

5 Donnelly, J. E., N. P. Pronk, D. J. Jacobson, S. J. Pronk, J. M. Jakicic. Effects of a very-low-calorie diet and physical-training regimens on body composition and resting metabolic rate. Am. J. Clin. Nutr. 54:56-61, 1991.

6 Fürst, P. Intracellular muscle free amino acids-their measurement and function. Proc. Nutr. Soc: 42:451-462, 1983.

7 Garby, L., J. S. Garrow, B. Jorgensen, et al. Relation between energy expenditure and body composition in man: specific energy expenditure in vivo of fat and fatfree tissue. Eur. J. Clin. Nutr. 42:301-305, 1987.

8 Hill, J. O., D. G. Slundt, T. S. Sbrocco, et al. Evaluation of an alternating-calorie diet with and without exercise in the treatment of obesity. Am. J. Clin. Nutr. 50:248-254, 1989.

9 Katch, F. I., S. S. Drumm. Effects of different modes of strength training on body composition and anthropometry. Clin. Sport Med. 5(3):413-459, 1986.

10 Keim, N. L., T. F. Barbieri, M. D. Van Loan, B. L. Anderson. Energy expenditure and physical performance in overweight women: response to training with and without caloric restriction. Metabolism 39:651-658, 1990.

11 Lemons, A. D., S. N. Kreitzman, A. Coxon, A. Howard. Selection of appropriate exercise regimes for weight reduction during VLCD and maintenance. Int. J. Obesity 13:119-123, 1989.

12 Lennon, D., F. Nagle, F. Stratman, E. Shrago, S. Dennis. Diet and exercise training effects on resting metabolic rate. Int. J. Obesity 9:39-47, 1984.

13 Molé, P. Impact of energy intake and exercise on resting metabolic rate. Sports Med. 10:72-87, 1990. 
14 Murgatroyd, P., W. Coward. An improved method for estimating changes in whole-body fat and protein mass in man. Brit. J. Nutr. 62:311-314, 1989.

15 Poehlman, E. T. A review: exercise and its influence on resting energy metabolism in man. Med. Sci. Sports Exerc. 21(5):515-525, 1989.

16 Poehlman, E. T., A. W. Gardner, P. A. Ades, et al. Resting energy metabolism and cardiovascular disease risk in resistance-trained and aerobically trained males. Metabolism 41(12):1351-1360, 1992.

17 Poehlman, E. T., E. S. Horton. The impact of food intake and exercise on energy expenditure. Nutrition reviews 47:129-137, 1989.

18 Poehlman, E. T., C. L. Melby, M. I. Goran. The impact of exercise and diet restriction on daily energy expenditure. Sports Med. 11:78-101, 1991.

19 Ravussin, E., C. Bogardus. Relationship of genetics, age, and physical fitness to daily energy expenditure and fuel utilization. Am. J. Clin. Nutr. 49:968-975, 1989.

20 Schoeller, D., E. Santen, D. Peterson, W. Dietz, J. Jaspen. Total body water measurement in humans with $18-\mathrm{O}$ and $2-\mathrm{H}$ labeled water. Am. J. Clin. Nutr. 33:2686-2693, 1980.

21 Tremblay, A., J.-P. Despres, C. Bouchard. The effects of exercise-training on energy balance and adipose tissue morphology and metabolism. Sports Med. 2:223-233, 1985.

22 Van Etten, L. M. L. A., F. T. J. Verstappen, K. R. Westerterp. Effect of body build on weight-training-induced adaptations in body composition and muscular strength. Med. Sci. Sports Exerc. 26(4):515-521, 1994.

23 Westerterp, K. R. Food quotient, respiratory quotient, and energy balance. Am. J. Clin. Nutr. 57 (suppl):759S-765S, 1993. 


\section{Chapter 4}

\section{Daily variation in energy expenditure during weight training versus continuous arm cranking and cycling}

Ludo M.L.A. van Etten, Klaas R. Westerterp and Frans T.J. Verstappen* Department of Human Biology and "Department of Movement Sciences, Maastricht University, Maastricht, The Netherlands Int. J. Sports Med. 17:497-503, 1996

\section{ABSTRACT}

To quantify day-to-day variability in energy expenditure ( $E E)$ during weight training, 10 male subjects completed 6 training sessions that consisted of a warming-up and cooling-down phase (10 min; arm cranking or cycling) and a computer-paced weighttraining phase using standard weights $(42 \mathrm{~min} ; 10$ exercises, $3 \times 15$ repetitions, work to rest ratio $=1: 1)$. The first 2 sessions were used to accommodate. During sessions 3-6 (labelled session A, B, C and D) cardiorespiratory response was continuously measured. Gas-exchange was converted to EE using the Weir formula. In the last two sessions training weights were raised by $\pm 50 \%$ and the initial warming-up and cooling-down exercise being arm-cranking was replaced by cycling. Mean EE was calculated for last five minutes of the warming-up (WU5) and cooling-down (CDS) and the complete weight-training phase (WT). Mean EE during WT for session A to D was $20.0 \pm 2.7,19.1 \pm 2.5,22.2 \pm 2.2$ and $21.9 \pm 2.4 \mathrm{~kJ} \cdot \mathrm{min}^{-1}$, respectively. Daily variation was tested by comparing test-retest phases $(A$ versus $B$ and $C$ versus $D$ ). Intra-individual variability in EE during respectively the lower (session $A$ vs $B$ ) and higher (session $C$ vs D) weight-training intensity was expressed as: absolute testretest difference $\left(0.9 \pm 0.5\right.$ and $\left.0.7 \pm 0.6 \mathrm{~kJ} \cdot \mathrm{min}^{-1}\right)$, difference relative to the mean of the rest-retest measurements $(2.3 \pm 1.4$ and $1.6 \pm 1.4 \%)$ and as the mean coefficient of variation (3.3 and $2.2 \%$ ). There were no differences in variability between both WTintensities nor exercise modes, irrespective of the way variability was expressed. In conclusion, intra-individual variability in EE during weight training does not differ from variability during arm cranking or cycling. 


\section{INTRODUCTION}

Since the early 1900's several methods including indirect calorimetry are in use to determine human energy expenditure. Knowledge about energy expenditure (EE) can be used in various ways. Scientists in the field of nutrition and dietitians use information on daily EE for dietary management of obesity or food supplementation. In sports sciences, a large amount of research is done on EE during endurance-type (steady-state) of activities such as running and cycling. These studies mainly focus on the predictive and diagnostic value of exercise cconomy since small differences in economy may determine competitive success within a homogeneous group of athletes (4).

Besides endurance-type of activities there are numerous sports activities that have an interval or intermittent character. Weight training is such a type of activity that alternates exercise with recovery-intervals. An increasing number of enthusiasts perform this type of sport/leisure-time activity to improve their health and fitness status or for cosmetics reasons. However, it is difficult to make general statements about the energy cost of weight training or to compare different studies because the extensive number of different exercises, as well as variations in the work/rest ratio, make almost every program unique. Notwithstanding this limitation, several investigators studied energy expenditure during weight training. Mainly these studies focussed on the metabolic cost of weight training $(6,9,10,14,18)$ but also the effects of different muscle contractions (5) and training intensity $(7,8)$ were studied. The study of Dudley (5) illustrates an uncommon application of such a study outcome. They recommend eccentric actions in excrcise prescription during space travel because these actions enhance resistance training-induced increases in strength with minimal additional energy cost.

If, however, the efficacy of an intervention aimed at exercise economy is to be evaluated, information on daily variation in EE during exercise is essential (12). In the case of running, for instance, the mean coefficient of variation in running economy is determined over a wide range of speeds and varies from 1 to $4 \%(2,11,17)$. Little is known about the daily variation of $\mathrm{EE}$ during weight training. The exercise-induced respiratory response, used to determine $\mathrm{EE}$, differs considerably between weight training and running. During intermittent exercise, respiratory adaptation and recovery alternate constantly whereas continuous exercise (i.e., running) results in a kind of steady state. This could affect daily variation in weight-training economy. Therefore, the present study was conducted to quantify day-to-day variability in EE during weight training.

A group of 10 trained male subjects completed six training sessions. Each session consisted of an endurance type of activity (arm cranking or cycling) during the warming-up and cooling-down phase and an intermittent phase that contained 10 weight-training exercises. Energy expenditure was measured with open circuit spirometry during the last four sessions. Since weight training contains both upper body and leg cxurciscs, daily variation in EE during weight training was compared with day-to-day variability during continuous arm cranking and leg cycling. 


\section{METHODS}

Subjects. Ten healthy male subjects, age between 22 and $39 \mathrm{yr}$, all participating in a weight-training program for at least $2 \mathrm{yr}$, were recruited from various fitness centers. The physical characteristics are presented in Table 4.1. After obtaining informed consent, each subject completed six training sessions, an isokinetic strength test and a hydrostatic weighing.

\begin{tabular}{|c|c|c|}
\hline Variables & mean & $\pm S D$ \\
\hline age (yr) & 30.9 & \pm 5.1 \\
\hline weight (kg) & 76.9 & \pm 6.7 \\
\hline height (m) & 1.81 & \pm 0.05 \\
\hline Body Mass Index $\left(\mathrm{kg} \cdot \mathrm{m}^{-2}\right)$ & 23.4 & \pm 2.2 \\
\hline fat $(\%)$ & 13.2 & \pm 5.5 \\
\hline fat-free mass (kg) & 66.6 & \pm 6.0 \\
\hline fat mass $(\mathrm{kg})$ & 10.3 & \pm 4.5 \\
\hline
\end{tabular}

Training sessions. Each total training session (TOTAL) was composed of an endurance type of activity (arm or bicycle ergometry for $10 \mathrm{~min}$ ) during the warmingup and cooling-down phase and an interval/intermittent weight-training phase (WT). The last five minutes of the warming up and cooling down were considered steadystate periods (WU5 and CD5, respectively). The first four sessions used arm ergometry (i.e., arm cranking; 48 watt $160 \mathrm{rpm}$, Ergostat Universal, Switzerland) as warming-up (WU5) and cooling-down (CD5) exercise. whereas session five and six used bicycle ergometry (i.e., cycling; 150 watt 75 rpm, Jaeger ER800). During weight training. standard weights were used. In session 5 and 6 these initial weights were raised by approximately 50\% (see Table 4.2). The selection of the standard weights was based on the lowest estimated level of training to ensure that all subjects could perform the exercises.

The WT phase included 3 sets of 15 repetitions of the following exercises: bench press, lunges, dumbbell pull, squat, and the overhead lats pulley. This sequence of exercises was completed twice to increase the volume of the session without increasing the number of different exercises. Total duration of the WT phase was 42 minutes. The ratio of work to rest was $1: 1$ ( $21 \mathrm{~min}$ work $+21 \mathrm{~min}$ rest; on average 34.5 sec to complete 15 reps). Since a single repetition of the lunges consisted of an alternating left and right step forward $(=2 \times 15$ reps), total duration of this exercise was approximately twice the time used by other exercises. Hence, exercise time was equally split between upper and lower body. A computer-generated acoustic signal dictated the pace during exercise and the duration of the resting intervals. Inter- and 
intra-individual variability in technical performance were minimized by using strict technique demands:

- bench press: barbell, standard hand spacing, from straight arm position to the chest, supine position during rest interval

- lunges: barbell, stride length $87 \mathrm{~cm}$ touching a cushioned vertical board with the shoe and knee, sitting during rest

- dumbbell pull: prone position on a bench, both dumbbells simultaneously tapping the floor or underside of the bench, prone position during rest

- squat: barbell, from standing position touching the seat of chair (height-adjusted to the medial joint cleft of the knee) with the buttocks, standing during rest

- overhead lats pulley: pulley device, sitting, wide grip, from straight arm position downwards touching the neck, sitting during rest

Before and after the WT phase subjects had one minute to change equipment. This made a total exercise duration of 63.5 minutes per session including rest time between sets.

Table 4.2. Protocol and sequence of the six training sessions.

\begin{tabular}{|c|c|c|c|c|c|c|c|}
\hline & Exercise & $\begin{array}{c}\text { session } \\
1 \\
\end{array}$ & $\begin{array}{c}\text { session } \\
2 \\
\end{array}$ & $\begin{array}{l}\text { session } \\
3(\mathrm{~A})\end{array}$ & $\begin{array}{l}\text { session } \\
4 \text { (B) }\end{array}$ & $\begin{array}{l}\text { session } \\
5(\mathrm{C})\end{array}$ & $\begin{array}{l}\text { session } \\
6 \text { (D) }\end{array}$ \\
\hline \multicolumn{2}{|c|}{ warming up (10 min) } & cranking & cranking & cranking & cranking & cycling & cycling \\
\hline & bench press $(\mathrm{kg})$ & 10 & 10 & 10 & 10 & 15 & 15 \\
\hline & lunges (kg) & 25 & 25 & 25 & 25 & 35 & 35 \\
\hline \multirow[t]{3}{*}{$2 x$} & dumbbell pull (kg) & $2 \times 4$ & $2 \times 4$ & $2 \times 4$ & $2 \times 4$ & $2 \times 5^{1 / 2}$ & $2 \times 5 \frac{1}{2}$ \\
\hline & squat (kg) & 30 & 30 & 30 & 30 & 45 & 45 \\
\hline & lats pulley (kg) & $141 / 4$ & $141 / 4$ & $141 / 4$ & $141 / 4$ & 20 & 20 \\
\hline \multicolumn{2}{|c|}{ cooling down (10 $\mathrm{min})$} & cranking & cranking & cranking & cranking & cycling & cycling \\
\hline
\end{tabular}

The duration for both warming-up and cooling-down was 10 minutes. The load and pedalling rate during cycling and arm-cranking were 150 watt $75 \mathrm{rpm}$ and 48 watt $60 \mathrm{rpm}$, respectively. The duration of the weight-training phase was 42 minutes. Training weights are expressed in $\mathrm{kg}$. Sessians 1 and 2 were used lor accommodation. During session 3-6 (A to D) cardiorespiratory data were collecled.

Session measurements. The first two sessions were used to accommodate the subjects to the laboratory setting and computerized pacing. No measurements were carried out and the subjects had full freedom of movement since they were not connected to the gas analyzer. The remaining four sessions (labelled session A, B, C and D) were all performed within a period of two weeks. Each training experiment was conducted at the same time in the morning to minimize the residual effects of physical activity, food intake and circadian variation, on energy expenditure. Furthermore, subjects were asked to refrain from any exercise 36 hours before each session and to eat similar brcakfasts. Prior to every session subjects rested supine on a bed for fifteen minutes to create comparable starting conditions. After the cooling down subjects were asked to sit as calm as possible for five minutes followed by another ten minutes of supine rest. 
Heart rate (HR) was measured continuously (Polar Sport Tester PE3000) including pre- and post-session resting periods. Pre- and post-training heart rates are defined as the mean HR over the last five minutes of the 15 minutes resting periods before and after exercise. Gas-exchange was continuously determined by open circuit spirometry using an automated system (Oxycon Beta, Mijnhardt). Gas analysis started simultaneously with the warming-up exercise and stopped after the five minutes of rest in a sitting position. Analyzers were calibrated before each experiment with standard gas mixtures. Gas-exchange was converted to energy expenditure using the Weir (15) formula.

Body composition. All body characteristics were taken the morning after a controlled overnight stay at the university where subjects abstained from eating for 14 hours. Body mass was measured after emptying the bladder to an accuracy of $\pm 5 \mathrm{~g}$ on an electronic scale (Mettler, E1200). Body density was measured using the hydrostatic weighing technique with simultaneous measurement of residual lung volume with the helium dilution technique (Volugraph 200, Mijnhardt). Water density was corrected for temperature. Percent fat was calculated from body density assuming a FFM and FM density of 1.097 and $0.901 \mathrm{~kg} \cdot l^{-1}$, respectively.

Muscular strength. No facilities were in the laboratory to sately determine individual IRM-values for each of the five exercises. To get some information about inter-individual variability in strength, muscular strength in the upper thigh and shoulder/arm was measured using an isokinetic dynamometer (Cybex II): knee extension at 2.094 $\mathrm{rad} \cdot \mathrm{s}^{-1}\left(=120^{\circ} \cdot \mathrm{s}^{-1}\right)$; shoulder/arm during a bench press movement at $1.047 \mathrm{rad} \cdot \mathrm{s}^{\cdot 1}$ $\left(60^{\circ} \cdot \mathrm{s}^{-1}\right)$. Subjects performed one set of five repetitions at each velocity from which the peak torque was noted as the parameter for maximal strength.

Statistical analyses. Paired $t$-tests were used to analyze differences between repeated measurements. Daily variablity was expressed as the absolute and relative test-retest differences, the coefficient of variation and the intraclass correlation coefficient. Because variability is expressed in absolute or relative (\%) terms, the original negative test-retest differences are changed into positive values affecting the assumed normal distribution. Hence, non-parametric tests were performed to compare daily variability: a Wilcoxon Matched-Pairs Signed-Rank Test to test low WT-intensity versus high WT-intensity and arm cranking versus cycling; a Friedmann Two-way Anova was used to compare variability between the warming-up, weight-training and coolingdown phase within one intensity. Statistical significance was set at $\mathrm{P}<0.05$.

\section{RESULTS}

Pattern of the EE and HR response. When the results of EE and HR are both plotted in the same figure (see Fig. 4.I) the identical pattern of both parameters is clearly visible. The first and last plateau mark the warming-up and cooling-down phase. 
Test-retest analysis on EE and HR was only performed within one intensity (testretest: lower intensity: session A versus B, and the higher intensity: session C vs D).

Inter-individual variation in isokinetic strength during leg extension and bench press was approximately $55 \%$ ( $205.6 \pm 35.6$ and $465.0 \pm 68.3 \mathrm{Nm}$, respectively).

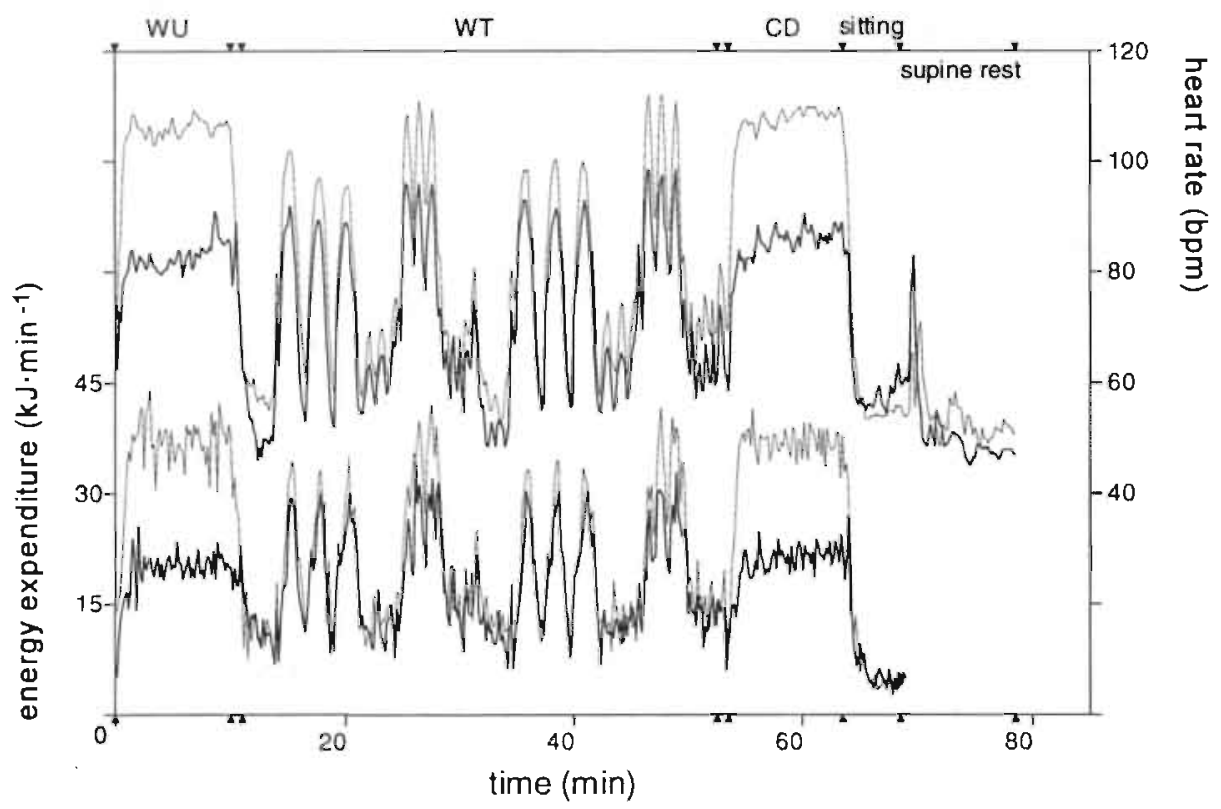

Fig. 4.1. Energy expenditure (lower lines) and heart rate (upper lines) of the same subject during training session $B$ (bold line) and $C$ (thin line). Triangles mark the beginning and end of the warming-up (WU), weight-training (WT) and cooling-down (CD) phases and the sitting and supine intervals of the post-training period.

Energy expenditure. Data on EE during the different phases of each session are presented in Table 4.3. A paired $t$-test between session A and B revealed a significantly reduced EE during session $\mathrm{B}$ with respect to the TOTAL and the WT phase ( $t$-value $=$ 3.89 and 4.70 , respectively; $\mathrm{P}<0.005$, see Table 4.3). Mean EE during WU5 and CD5 did not statistically differ between the sessions $A$ and $B$. There were no differences in EE during WU5, WT, CD5 and TOTAL between session C and D.

The RER's did not statistically differ between the test-retest sessions (mean session $\mathrm{A} / \mathrm{B}: \mathrm{WT}=0.92 \pm 0.04$. WU5 $=0.98 \pm 0.05, \mathrm{CD} 5=0.96 \pm 0.05$, TOTAL $=0.94 \pm 0.04$; mean session $\mathrm{C} / \mathrm{D}: \mathrm{WT}=0.98 \pm 0.04$, WU5 $=0.93 \pm 0.04, \mathrm{CD} 5=0.90 \pm 0.03$, TOTAL $=0.95$ \pm 0.04 ).

Heart rate. Non-exercise HR (pre- and post-training $\mathrm{HR}$ ) as well as heart rate during the WT and TOTAL phase differed significantly between session A and B. In all cases HR during session A was higher. HR during WU5 and CD5 did not differ. There were no differences in HR between sessions $\mathrm{C}$ and D (see Table 4.3). 
Table 4.3. Energy expenditure and heart rate (means $\pm S D$ ) during the total session (TOTAL) and the subintervals: last five minutes of the warming-up (WU5), weight-training phase (WT), and the last five minutes of the cooling-down (CD5).

\begin{tabular}{|c|c|c|c|c|}
\hline Interval & session $\mathrm{A}$ & session B & session C & session D \\
\hline \multicolumn{5}{|c|}{ energy expenditure ( $\left.\mathrm{kJ} \cdot \mathrm{min}^{-1}\right)$} \\
\hline TOTAL & $20.7 \pm 2.3$ & $20.0 \pm 2.2^{\star \star *}$ & $27.2 \pm 1.8$ & $27.1 \pm 2.0$ \\
\hline WU5 & $22.9 \pm 1.6$ & $22.4 \pm 1.9$ & $39.2 \pm 1.1$ & $39.1 \cdot \pm 1.5$ \\
\hline WT & $20.0 \pm 2.7$ & $19.1 \pm 2.5^{\star \star}$ & $22.2 \pm 2.2$ & $21.9 \pm 2.4$ \\
\hline CD5 & $23.7 \pm 1.8$ & $23.6 \pm 2.4$ & $40.0 \pm 1.7^{6}$ & $40.3 \pm 2.0^{8}$ \\
\hline \multicolumn{5}{|c|}{ heart rate (beats. $\mathrm{min}^{-1}$ ) } \\
\hline Pre-training & $64.2 \pm 9.4$ & $60.6 \pm 6.9^{*}$ & $60.7 \pm 8.6$ & $61.3 \pm 9.6$ \\
\hline TOTAL & $103.3 \pm 18.1$ & $98.9 \pm 16.6^{\star}$ & $115.9 \pm 18.2$ & $113.4 \pm 15.4$ \\
\hline WU5 & $109.9 \pm 18.7$ & $107.5 \pm 18.6$ & $127.1 \pm 12.2$ & $125.7 \pm 11.5$ \\
\hline WT & $100.7 \pm 18.7$ & $95.3 \pm 16.8^{*}$ & $109.8 \pm 20.4$ & $107.0 \pm 17.2$ \\
\hline CD5 & $116.4 \pm 19.8^{5 \$}$ & $113.8 \pm 20.3^{56}$ & $139.2 \pm 19.3^{55}$ & $136.4 \pm 15.9^{55}$ \\
\hline Post-training & $65.2 \pm 10.7$ & $62.7 \pm 11.5^{*}$ & $69.9 \pm 13.8^{\S}$ & $68.8 \pm 13.3^{5}$ \\
\hline
\end{tabular}

The asterisk ( $\star$ ) symbolizes significant test-retest differences whereas the section sign (\$) symbolizes within session differences of WU5 versus $C D 5$, and pre- versus post-training values (level of significance: ${ }^{*} P<0.05, \cdots P<0.005,{ }^{6} P<0.05$ and ${ }^{\S} \mathrm{P}<0.005$ )

Daily variation in EE. To obtain comprehensive information on the daily variation in $\mathrm{EE}$ during weight training, variability was expressed in four different ways (see Table 4.4): as absolute difference (in $\mathrm{kJ} \cdot \mathrm{min}^{-1}$ ) between test-retest sessions, as percentage of the mean test-retest value (day-to-day variation), as the mean coefficient of variation (=the mean of individual SD/ $\bar{x} \times 100$ ) and the test-retest intraclass correlation coefficient.

Mean absolute differences in EE during WT were $0.9 \pm 0.5 \mathrm{~kJ} \cdot \mathrm{min}^{-1}$ (range $=0.0-1.6$ $\mathrm{kJ} \cdot \mathrm{min}^{-1}$ ) and $0.7 \pm 0.6 \mathrm{~kJ} \cdot \mathrm{min}^{-1}$ (range $=0.0-2.0 \mathrm{~kJ} \cdot \mathrm{min}^{-1}$ ) for sussions $\mathrm{A} / \mathrm{B}$ and $\mathrm{C} / \mathrm{D}$, respectively. Mean day-to-day variation in EE during WT was $2.3 \pm 1.4$ and 1.6 $\pm 1.4 \%$ (range $=0.1-4.3$ and 0.1-5.0\%) for the lower and higher intensity. The corresponding values for the mean coefficient of variation were 3.3 (range $=0.2-6.1$ ) and 2.2 (range $=0.1-7.0$ ). All test-retest phases except WU5 of session C and D were significantly correlated.

A Friedmann Two-way Anova revealed no significant differences in variability between the phases within one session irrespective the way daily variability was expressed. Comparison of variability of the phases between session $A / B$ and session $\mathrm{C} / \mathrm{D}$ revealed a significant smaller day-to-day variation and coefficient of variation of the $\mathrm{CD} 5$ phase during cycling (session $\mathrm{C} / \mathrm{D}$ ). To examine whether variability was a function of $\mathrm{EE}$, the difference between the repeated sessions $(\Delta \mathrm{EE})$ was plotted against the mean of test-retest values (see Fig. 4.2, Bland-Altman plot)(1). There was no significant relation between $\triangle \mathrm{EE}$ and the mean of both sessions. 


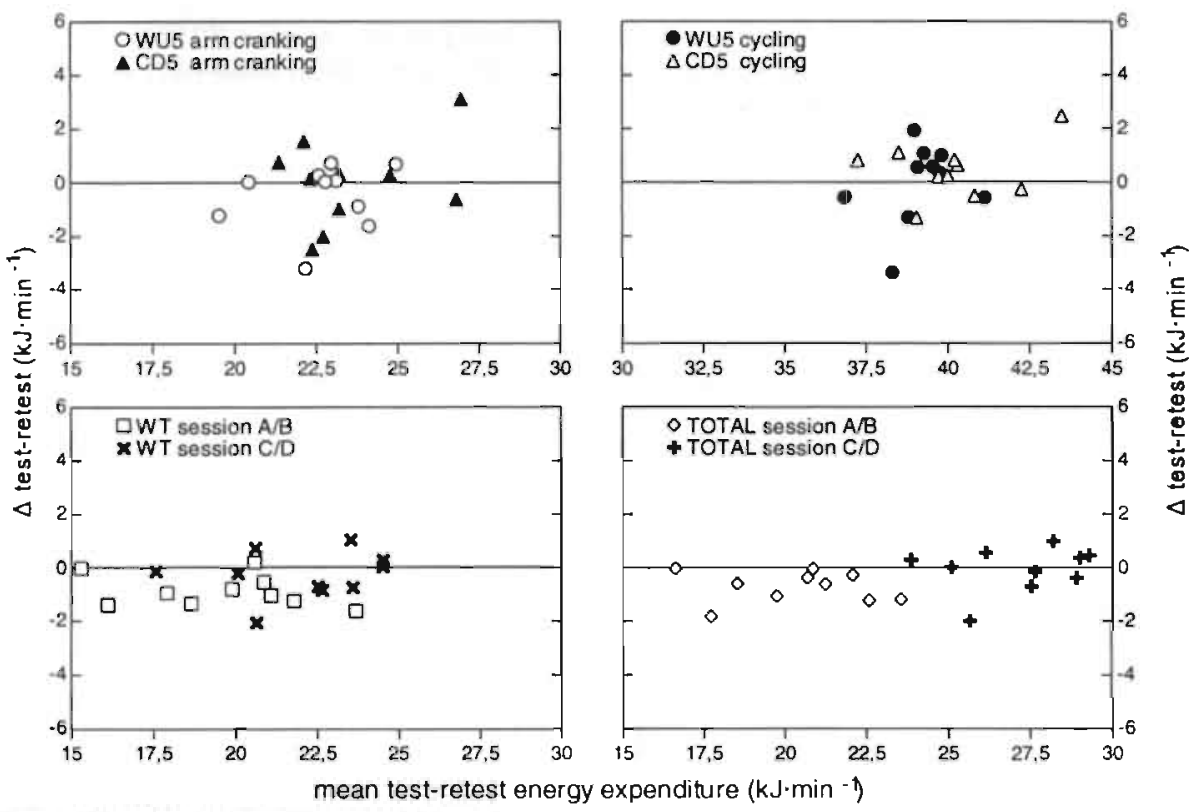

Fig. 4.2. Test-retest differences (second minus first measurement) in energy expenditure plotted against mean lest-retest energy expenditure during the three different exercise modes: arm cranking, cycling, weight training (WT) and the total (TOTAL) training session.

Table 4.4. Mean absolute difference $\left(\mathrm{kJ} \cdot \mathrm{min}^{-1}\right)$, day-to-day variation (\%), mean coefficient of variation and Intraclass correlation coefficient of energy expenditure between the various repeated session phases.

\begin{tabular}{llllll}
\hline & sesslon & TOTAL & WU5 & WT & CD5 \\
\cline { 2 - 6 } Absolute differences & 3 vs 4 & $0.7 \pm 0.6$ & $0.9 \pm 1.0$ & $0.9 \pm 0.5$ & $1.2 \pm 1.0$ \\
$\left(\mathrm{~kJ} \cdot \mathrm{min}^{-1}\right)$ & 5 vs 6 & $0.6 \pm 0.6$ & $1.1 \pm 0.9$ & $0.7 \pm 0.6$ & $0.8 \pm 0.7$ \\
Day-to-day & 3 vs 4 & $1.8 \pm 1.5$ & $1.9 \pm 2.2$ & $2.3 \pm 1.4$ & $2.6 \pm 2.1$ \\
variation (\%) & 5 vs 6 & $1.1 \pm 1.1$ & $1.4 \pm 1.2$ & $1.6 \pm 1.4$ & $1.0 \pm 0.8^{*}$ \\
Mean coefficient & 3 vs 4 & 2.5 & 2.8 & 3.3 & 3.7 \\
of variation (\%) & 5 vs 6 & 1.5 & 2.0 & 2.2 & $1.4^{\star}$ \\
Intraclass correlation & 3 vs 4 & $0.966^{55}$ & $0.750^{5}$ & $0.975^{55}$ & $0.696^{5}$ \\
coefficients & 5 vs 6 & $0.903^{55}$ & 0.369 & $0.926^{55}$ & $0.859^{55}$ \\
\hline
\end{tabular}

There are no differences in variability between the different weight-training phases. The asterisk $(\star)$ symbolizes significant $(P<0.05)$ differences between the lower and the higher intensity. The section sign $(\S)$ symbolizes the level of significance of the intraclass correlation coefficient $\S P<0.05, \S \S P<0.001$.

Within session differences between the WU5 and CD5 phase. EE during the CD5-phase of sessions C and D was significantly increased (see Table 4.3) compared with the within-session WU5-value (40.0 vs $39.2 \mathrm{~kJ} \cdot \mathrm{min}^{-1}$ and $40.3 \mathrm{vs} 39.1 \mathrm{~kJ} \cdot \mathrm{min}^{-1}$, 
respectively; $t$-value $=2.37$ and $2.95, \mathrm{P}<0.05)$. CD5-values of sessions $\mathrm{A}$ and $\mathrm{B}$ showed the same trend but did not statistically differ from WU5 $(t$-value $=2.11$ and $2.03, \mathrm{P}=$ 0.064 and 0.073 , respectively).

HR-values during CD5 of all sessions were elevated in comparison with their corresponding WU5-HR.

RER-values decreased significantly $(\mathrm{P}<0.005)$ during CD5 except for session $B$ $(\triangle \mathrm{RER}=-0.029 \pm 0.02,-0.013 \pm 0.02,-0.036 \pm 0.02$ and $0.030 \pm 0.02$ for session $\mathrm{A}-\mathrm{D}$; $t$-value $=4.44,1.89,4.54$ and 6.15 , respectively). This decrease in RER showed no relationship with the corresponding EE-differences.

\section{DISCUSSION}

Test-retest differences. The aim of this study was to quantify daily variability in EE during weight training with arm cranking and cycling as a reference. A lundamental necessity to quantify variability is the absence of a test order effect, i.e., there should be no significant differences between repeated measurements. Analysis revealed a significant difference between sessions $\mathrm{A}$ and $\mathrm{B}$ with respect to the TOTAL and the WT phase (see Fig. 4.2). During session B, nine out of ten subjects used less energy during the WT phase, while all subjects used less during the TOTAL phase. This high systematic difference in $\mathrm{EE}\left(0.9 \pm 0.5 \mathrm{~kJ} \cdot \mathrm{min}^{-1} ; \mathrm{P}<0.005\right)$ suggests that there were general factors that modified EE. An improvement in technical skill is not likely since all subjects had for at least 2 yr weight-training experience. Confounding by technological fluctuation is unlikely since all measurements on one subject were done within a two-wk time span, whereas three months were used to measure all subjects. Psychogenic factors like nervousness (3) caused by the unaccustomed spirometric connection, are more likely to explain part of the higher EE during the first measurement session. Although two sessions were used to accommodate subjects to the laboratory condition, subjects did not wear a mask nor were they limited in their movement by the laboratory setting (particularly during the WT phase) until session A. In a study on day-to-day variations in metabolic measurements, Amstrong (2) reported a consistent trend of a higher oxygen consumption during the first measurements of cycling and running experiments. Previous experience with the testing apparatus was mentioned as a possible modifier. The higher pre-training and WT heart rates during session A seem to be consistent with this hypothesis. To avoid this potential complication by psychogenic factors it is advised to practise in the final setting.

Daily variation. Intra-individual variability in EE during weight training represents the sum of biological variation and technical error. Unfortunately, in this study there also appeared to be a test order effect. A systematic difference between measurements increases the magnitude of normal intra-individual variation. Therefore, variability during the WT and TOTAL phase of session A/B will probably be overestimated.

To compare variability of energy cost between various modes of exercise it is essential to look at the within-subject differences in several ways (see Table 4.4). First, 
absolute differences between two measurements expressed in terms of $\mathrm{kJ} \cdot \mathrm{min}^{-1}$ give information about the average size and range of variation that can be expected. Mean absolute differences of all defined intervals ranged from $0.6 \mathrm{~kJ} \cdot \mathrm{min}^{-1}$ to $1.2 \mathrm{~kJ} \cdot \mathrm{min}^{-1}$. Because within-subject differences may be a function of the magnitude of the energy cost, day-to-day variation should also be expressed relative (\%) to the mean EE. Expressing differences relative to the mean makes it also possible to compare data expressed in dissimilar physical units (e.g., $\mathrm{ml} \mathrm{O}_{2} \cdot \mathrm{min}^{-1} \cdot \mathrm{kg}^{-1} \mathrm{vs} \mathrm{kJ} \cdot \mathrm{min}^{-1}$ ). The mean day-to-day variation ranged from $1.0 \%$ to $2.6 \%$. In studies on running economy, intraindividual variations in economy ranged from $1.6 \%$ up to $11.0 \%(12,13)$. This wide range in variation was ascribed to discrepancies between studies in controlling factors like testing experience, time of the day and training activity. Considering the range of variation in EE within this study, it may be concluded that variation relative to the mean of all intervals was well within the range of variation during endurance activities as reported in the literature. A third method to quantify variability is to express sample standard deviation relative to the mean of the sample (coefficient of variation). Mean coefficients of variation ranged from $1.5 \%$ to $3.6 \%$. Hence, EE-values can be expected to vary within subjects $\pm 1.5-3.6 \%$ in $68 \%$ of the tests and $\pm 3.0-7.2 \%$ in $98 \%$ of all tests. These results are in accordance with studies on running and cycling reporting mean coefficients of variations between $1.3-4.7 \%(2,13,17)$. A fourth method to express reliability is the intraclass correlation coefficient. Although a correlation coefficient gives only information about the strength of a relationship between two variables and not about the size of the differences between these variables, this method is often used in studies on variability. The association between the repeated measurements was high except for the WUIS interval of sessions C and D. Since the CD5 phase showed significant within-subject correlations using the same mode of exercise, this lack of significant correlation was apparently due to the smaller between-subject variation in EE during WU5.

To find out if weight-training intensity affected variability, the WT phases of session $\mathrm{A} / \mathrm{B}$ and $\mathrm{C} / \mathrm{D}$ were compared. Statistical analyses indicated that increasing intensity by raising training loads by $50 \%$ had no significant effect on variability irrespective of the way it was expressed. As mentioned before, variability during the WT phase of session $\mathrm{A} / \mathrm{B}$ could be overestimated due to the systematic test-retest difference. If individual values of session $A$ were corrected for the mean difference between session $\mathrm{A}$ and $\mathrm{B}$, the mean absolute difference, day-to-day variation and coefficient of variation decreased $\left(0.5 \pm 0.3 \mathrm{~kJ} \cdot \mathrm{min}^{-1}, 1.2 \pm 0.9 \%\right.$ and $1.8 \pm 1.3 \%$, respectively) but still did not differ from variability during session $C / D$. This supports the finding of Williams (17) who found no differences in coefficients of variation between three different speeds of running. However, the effect of intensity on variability can be biased by inter-individual differences in intensity (percentage of 1 repetition max) since weight-training loads in kilograms were identical for all subjects whereas interindividual variation in muscular strength during bench press and leg extension was approximately $\pm 55 \%$. On the other hand, due to the wide range of intensities that were included (even within one absolute load), generalizability of these data on variability increases. 
The difference in variability found between the cooling-down values of arm cranking and cycling (expressed as day-to day variation + coefficient of variation) is most probably due to local fatigue of the shoulder and arm muscles because warmingup values do not differ. Furthermore, the absolute differences of both modes of exercise are nearly equal whereas the magnitude of energy cost of cycling is almost twice the energy cost of arm cranking implicitly resulting in a larger relative test-retest difference.

Since no differences in variability existed between weight training, arm cranking and cycling irrespective of the way it was expressed, in addition to the fact that these results were in accordance with findings of other studies, it can be concluded that day to day variation in EE during intermittent weight training does not differ from continuous endurance exercises.

Within-session differences between WU5 and CD5. To examine the within-session effect of weight training on EE, HR and RER during steady state exercise, WU5 and CD5-values are compared assuming a comparable anaerobic energy contribution to the energy cost of the exercise. The consistent trend of an increase in EE and HR, and the decrease in RER during the cooling down shows a weight-training-induced effect on these variables. This change in EE, HR and RER is most pronounced during the highest intensity weight training suggesting that intensity could play a role. A proportional increased recruitment of Type $\Pi$ fibers is thought to be the major contributor to the increased EE (16). Other plausible factors that could mediate the increased energy requirements during the cooling-down exercise are an increased body temperature (I 1), blood lactate, circulating catecholamines and "extraneous work" by muscles not associated with external work.

The decrease in RER during the CD5 phase was probably due to a relative increase in fat oxidation, although this explanation conflicts somewhat with the earlier statement about the increased recruitment of Type II fibers during the CD5 phase. Other factors that could have contributed to the decreased RER during the cooling down are carbon dioxide retainment in the body to replenish the bicarbonate used to buffer lactic acid produced during the WT phase, or hypoventilation to reestablish diminished $\mathrm{PCO}_{2}$ levels due to hyperventilation during the WT phase.

Calculation of EE. Energy expenditure was calculated using the Weir (15) formula that includes oxygen consumption as well as carbon dioxide production. Most other studies on EE and weight training assume a constant $20.9 \mathrm{~kJ}$ (5 kcal) of energy released for each liter of oxygen consumed. Because RER's during WT are close to 1.0 the differences between the applied method and the more usual method are very small. Therefore one uniform method was preferred to calculate EE.

In summary, after comparing variability of indirect calorimetry during weight training with other modes of exercise within one subject, it can be concluded that no difference in variability exists between weight training (over a wide range of intensities) and 
cycling or arm cranking. Furthermore, EE and HR increased whereas RER decreased during arm cranking and cycling following a bout of weight training.

\section{REFERENCES}

1 Altman. D. G., J. M. Bland. Measurement in medicine: the analysis of method comparison studies. Statistican 32:307-317, 1983.

2 Armstrong, L., D. Costill. Variability of respiration and metabolism: responses to submaximal cycling and running. Rescarch Quarter/y 56:93-96, 1985.

3 Crews, D. J. Psychological state and running economy. Med. Sci. Sports Exerc: 24(4):475-482, 1992.

4 Daniels. J. T. A physiologist's view of running economy. Med. Sci. Sports Exerc. 17(3):332-338, 1985.

5 Dudley, G. A., P. A. Tesch, R. T. Harris, C. L. Golden, P. Buchanan. Influence of eccentric actions on the metabolic cost of resistance exercise. Aviat. SpacAviation, Space, and Environmental Medicinee Environ. 62:678-682, 1991.

6 Hickson, J. F., M. J. Buono, J. H. Wilmore, S. H. Constable. Energy cost of weight training exercise. Nat. Strength and Conditioning Assoc. J. 6(5):22-66, 1984.

7 Hunter, G., L. Blackman, L. Dunnam, G. Flemming. Bench press metabolic rate as a function of exercise intensity. J. Appl. Sport Sci. Res. 2(1):1-6, 1988.

8 Kalb, J. S., G. R. Hunter. Weight training economy as a function of intensity of the squat and overhead press exercise. J. Sports Med. Phys. Fitness 31:154-160, 1991.

9 Katch, F. I., P. S. Freedson, C. A. Jones. Evaluation of acute cardiorespiratory responses to hydraulic resistance exercise. Med. Sci. Sports Exerc. 17:168-173, 1985.

10 Liverman, R. D., C. Groden. Metabolic analysis of high intensity weight training. Abstract. Med. Sci. Sports Exerc. 14:169, 1982.

11 Morgan, D. W.. M. Craib. Physiological aspects of running economy. Med. Sci. Sports Exerc. 24(2):456-461, 1992.

12 Morgan, D. W., P. E. Martin, G. S. Krahenbuhl. Factors affecting running economy. Sports Med. 7:310-330, 1989.

13 Morgan, D. W., P. E. Martin, G. S. Krahenbuhl, F. D. Baldini. Variability in running economy and mechanics among trained male runners. Med. Sci. Sports Exerc. 23(3):378-383, 1991.

14 Scala, D. S., J. McMillan, D. Blessing, R. Rozenek, M. Stone. Metabolic cost of a preparatory phase of training in weight lifting: A practical observation. $J$. Applied Sports Science Research 1(3):48-52, 1987.

15 Weir de, J. B. New methods for calculating metabolic rate with special reference to protein metabolism. J. Physiol. 109: 1-9, 1949.

16 Whipp, B. J. The slow component of $\mathrm{O}_{2}$ uptake kinetics during heavy exercise. Med. Sci. Sports Exerc. 26(1 1):1319-1326, 1994. 
17 Williams, T. J., G. S. Krahenbuhl, D. W. Morgan. Daily variation in running economy of moderately trained male runners. Med. Sci. Sports Exerc. 23(8):944$948,1991$.

18 Wilmore, J., R. Parr, P. Ward, P. Vodak, T. Barstow. Energy cost of circuit weight training. Med. Sci. Sports Exerc. 10(2):75-78, 1978. 


\section{Chapter 5}

\section{Anthropometrical and physiological correlates of energy expenditure during weight training}

Ludo M.L.A. van Etten, Frans T.J. Verstappen*, Klaas R. Westerterp, Bart J.B. Boon and Wim. H.M. Saris.

Department of Human Biology and *Department of Movement Sciences, Maastricht University, Maastricht, The Netherlands

\section{ABSTRACT}

This study was performed to examine the relationship between energy expenditure (EE) during weight-training exercise at two intensities (EElow and EEhigh) and a selected number of physiological and anthropometrical variables. During both intensities EE was significantly related with exercise heart rate, sleeping metabolic rate, body mass, height, and fat-free mass. Using multiple regression analysis, the correlates body mass, heart rate and body composition (\% fat), explained $83 \%$ of the inter-subject variance in EElow $(37,40$ and $5 \%$, respectively). During the higher intensity training body mass and heart explained a total of $73 \%$ of the variability in EEhigh (32 and $41 \%$, respectively). Prediction of EE of several weight-training workouts described in the literature using the two multiple regression equations resulted in a consistent underestimation of actual measured EE. It was concluded that body mass together with heart rate explained more than $70 \%$ of the inter-subject variation in EE. However, this relationship between body mass, heart rate and EE is protocol specific. 


\section{INTRODUCTION}

Studies on correlates of energy expenditure (EE) during exercise examine the relationship between the energy cost of exercise and a number of variables. Although this statistical method quantifies the relationship, it provides no information on the causality of the relationship. Whereas some correlates are the cause of variation in $\mathrm{EE}$, others will be the result of variation in $\mathrm{EE}$.

Correlates that cause variation in the energy cost of exercise are named determinants of EE. The most obvious determinant of EE during physical exercise is exercise intensity. This is illustrated by the linear relationship between EE and running speed or power output during cycling (both endurance types of activities) (2). Based on this relationship, these determinants can be used to estimate EE. Like determinants of EE, also the correlates that are the result of changes and/or differences in EE can be used to estimate EE. The most applied estimate of energy expenditure is heart rate (HR). Heart rate monitoring is used to estimate EE in a variety of activities (e.g.. during sports, professional as well as daily activities) $(5,8,11,18-20,22-24)$. Since both types of correlates explain part of the variance in EE between subjects, also a combination of these different types of correlates can be used to estimate EE during physical exercise (i.e., predictors).

During endurance type of activities (e.g., running or cycling) exercise intensity, as the most important determinant of the energy cost of exercise, can be expressed relative to maximal performance (percentage of $\mathrm{VO}_{2} \max$, cycling power or maximal running speed). In contrast to endurance exercise, it is considerably more complex to determine the exercise intensity during an intermittent type of exercise like weight training. A frequently used method to define weight-training intensity is to express the training load (i.e., training weights, resistance) relative to the one repetition maximum. However, factors like work/rest-ratio and the addition of upper body work will substantially affect exercise intensity and the relationship between energy expenditure and some of the correlates. Using the same resistance, a shorter rest-interval will increase exercise intensity whereas a longer rest-interval will decrease weight-training intensity. Upper body work in comparison with lower body work demands a higher $\mathrm{VO}_{2}$ (i.e., energy expenditure) and heart rate at a given absolute submaximal workload $(25,27)$. Nevertheless, research revealed that in line with endurance exercise. energy expenditure during weight training also increases as training intensity increases. From the literature there are indications that the within subject relationship between training load and energy expenditure is not linear but exponential ( $\Delta$ energy expenditure $>$ $\Delta$ load) $(13,15)$. The within group relationship, however, appears to be linear $(5)$.

Besides exercise intensity it is obvious that there need to be additional determinants of the energy cost of exercise because substantial differences in energy expenditure are found between individuals performing the same work during endurance as well as weight-training activities $(21,28)$. In both cross-sectional and longitudinal EE-studies knowledge about these determinants is crucial when they differ between groups or are likely to change during an intervention. 
The purpose of the present study was to examine the relationship between a selection of physiological and anthropometrical variables and the energy cost of weight training. The variables included in the analysis were exercise and resting heart rate, muscular strength, sleeping metabolic rate and the anthropometrical variables: body mass, height, \%fat, fat-free mass and fat mass. The rationale to include variables was based on a generally accepted physiological and mechanical relationship with energy expenditure. Because the correlates were mutually related, the correlates were included in a multiple regression analysis to quantify their cumulative contribution to the inter-subject variation in the energy cost of the used weight-training workouts. To examine whether the arithmetically determined relationship can generally be applied to predict EE during weight training, the equation is validated using data from other weight-training studies found in the literature.

\section{METHODS}

Subjects. Thirty-five healthy males were recruited to participate in this study. Detailed information over the purpose and all methods used was provided and written informed consent was obtained. The subjects had to perform six standardized training sessions using two standard training loads (i.e., two intensities). From the initial group of 35 subjects, 6 subjects were excluded because they were not able to perform the sessions using the prescribed standard load during the higher intensity weight-training sessions. The remaining group of 29 subjects consisted of 8 subjects with several years of weight-training experience, 14 subjects that had participated in an 18 wk weighttraining program and 7 subjects with no previous weight-training experience.

Weight-training sessions. To determine the energy cost of two wcight-training intensities, a total of six weight-training sessions was performed in the laboratory. Each session included a weight-training phase, a warming-up phase, and a cooling-down phase. The weight-training phase consisted of 3 sets of 15 repetitions of the following exercises: bench press, lunges, dumbbell pull, squat, and the overhead lats pulley ( 3 upper body and 2 lower body exercises) using standard weights. This sequence of five exercises was completed twice to increase the volume of the session without increasing the number of different exercises (see Table 5.1). Total duration of the weight-training phase was 42 minutes. Since a single repetition of the lunges consisted of an alternating left and right step forward, total duration of this exercise was approximately twice the time used by other exercises. Hence, exercise time was equally split up between upper and lower body work. The work to rest ratio was 1:1. A computer-generated acoustic signal dictated the pace during exercise and the duration of the resting intervals. Variability in technical performance was minimized by using strict technique demands (28). During session 5 and 6 (higher weight-training intensity) the initial training load used during session 1 to 4 (lower weight-training intensity) was increased by raising training weights by approximately $50 \%$. As is customary, subjects also performed a warming-up and cooling-down (both $10 \mathrm{~min}$ ). 
During the first four sessions arm ergometry (i.e., arm cranking; 48 watt/ $60 \mathrm{rpm}$, Ergostat Universal, Switzerland) was used, whereas session five and six used leg ergometry (i.e., cycling; 150 watt/ 75 rpm, Jaeger ER800) as warming-up and coolingdown exercise.

Table 5.1. Protocol and sequence of the six training sessions.

\begin{tabular}{|c|c|c|c|c|c|c|c|}
\hline & Exercise & $\begin{array}{c}\text { session } \\
1 \\
\end{array}$ & $\begin{array}{c}\text { session } \\
2 \\
\end{array}$ & $\begin{array}{c}\text { session } \\
3 \\
\end{array}$ & $\begin{array}{c}\text { session } \\
4 \\
\end{array}$ & $\begin{array}{c}\text { session } \\
5 \\
\end{array}$ & $\begin{array}{c}\text { session } \\
6 \\
\end{array}$ \\
\hline \multicolumn{2}{|c|}{ warming up (10 min) } & cranking & cranking & cranking & cranking & cycling & cycling \\
\hline & bench press $(\mathrm{kg})$ & 10 & 10 & 10 & 10 & 15 & 15 \\
\hline & lunges $(\mathrm{kg})$ & 25 & 25 & 25 & 25 & 35 & 35 \\
\hline \multirow[t]{3}{*}{$2 x$} & dumbbell pull (kg) & $2 \times 4$ & $2 \times 4$ & $2 \times 4$ & $2 \times 4$ & $2 \times 51 / 2$ & $2 \times 5 \frac{1}{2}$ \\
\hline & squat $(\mathrm{kg})$ & 30 & 30 & 30 & 30 & 45 & 45 \\
\hline & lats pulley $(\mathrm{kg})$ & $14 \%$ & $141 / 4$ & $14 \frac{1}{4}$ & $14 \frac{1}{4}$ & 20 & 20 \\
\hline \multicolumn{2}{|c|}{ cooling down (10 $\mathrm{min})$} & cranking & cranking & cranking & cranking & cycling & cycling \\
\hline
\end{tabular}

Energy expenditure, and heart rate measurements. From the total of 6 training sessions, session 1 and 2 were used to accommodate the subjects to the laboratory setting and computerized pacing and no measurements were carried out. The remaining four measurement sessions consisted of two sessions performed at the lower weighttraining intensity (session 3 and 4 ) and two sessions performed at the higher weighttraining intensity (session 5 and 6). The average energy cost of the two lower intensity sessions (EElow) and the average energy cost of the two higher intensity sessions (EEhigh) were included in the final analysis. All sessions were conducted at the same time in the morning to minimize the residual effects of physical activity, food intake and circadian variation, on energy expenditure. Furthermore, subjects were asked to refrain from any exercise or strenuous physical activity 36 hours before each session and to eat similar breakfasts.

To create similar starting conditions, subjects were asked to sit relaxed for five minutes followed by ten minutes of supine rest prior to every session. Data on gasexchange and heart rate were continuously collected during the entire experiment (including the resting period). Resting heart rate was defined as the lowest $2 \frac{1}{2} \mathrm{~min}$ value over the entire $15 \mathrm{~min}$ pre-session period. Gas-exchange was measured by open circuit spirometry using an automated system (Oxycon Beta, Mijnhardt). Gas analyzers were automatically calibrated before each experiment with standard gas mixtures. Gas-exchange was converted to energy expenditure using the Weir formula (29). Heart rate was monitored using a Polar Sport Tester PE3000 and recorded over 10 second intervals (beats. $\mathrm{min}^{-1}$ ).

Sleeping metabolic rate (SMR). SMR was measured during an overnight stay in a respiration chamber (18.00-8.00 h). Dinner was used before entering the chamber and subjects were not allowed to eat until body composition was determined the next 
morning. To minimize the residual effects of training on energy expenditure, subjects were asked to refrain from any exercise or strenuous activity on the day prior to the measurement. Hence, SMR measurement was always $>8$ hours after the last meal and $>30$ hours after the last training session. Technical information on the respiration chamber is described elsewhere (6). SMR was defined as the lowest mean energy expenditure over a shiftable three hour interval between 0:00-6:00 am.

Physical characteristics. Anthropometrical measurements were taken the morning after the controlled stay at the laboratory. After voiding body mass was measured to an accuracy of $5 \mathrm{~g}$ on an electronic scale (Mettler, E1200). Body volume was determined using the hydrostatic weighing technique. Residual lung volume was simultaneously measured using the helium dilution technique (Volugraph 200, Mijnhart). Total body water (TBW) was measured using deuterium $\left({ }^{2} \mathrm{H}_{2} \mathrm{O}\right)$ dilution. Body composition was assessed using the three-component model of Siri that combines body density with TBW (9).

Muscular strength. To collect information on muscular strength in the leg and shoulder/arm an isokinetic dynamometer (Cybex II, Lumex Inc., New York) was used: knee extension at $2.094 \mathrm{rad} \cdot \mathrm{s}^{-1}\left(=120^{\circ} \cdot \mathrm{s}^{-1}\right)$; shoulder/arm during a bench press movement at $1.047 \mathrm{rad} \cdot \mathrm{s}^{-1}\left(60^{\circ} \cdot \mathrm{s}^{-1}\right)$. Subjects performed one set of five repetitions from which the peak torque was noted as the parameter for maximal strength. The decision to use the less exercise specific isokinetic method was mainly based on two considerations: a higher risk of injury when using an "isotonic" one repetition maximum test and the point that some participants had no previous weight-training experience (biasing effect of motor control).

Statistical analysis. Pearson correlation coefficients and stepwise multiple regression analysis were used to examine the relationship between the energy cost of weight training and the included hypothesized variables and to quantify the contribution of these variables to the inter-subject variation in energy expenditure during exercise. Statistical significance was set at $\mathrm{P}<0.05$.

\section{RESULTS}

Subjects characteristics and data on energy expenditure, heart rate, muscular strength and SMR are presented in Table 5.2. After increasing the training load by $50 \%$ the average energy cost of the training session increased by $12.3 \%$. The average intersubject variance relative to the average value $(=(\max -\min ) /($ average $\times 0.01), \%)$ was $45 \%$ at the lower and $36 \%$ at the higher weight-training intensity.

The inter-subject variance relative to the average value of the included correlates ranged from $12 \%$ for height to $148 \%$ for fat mass. The Pearson correlation coefficients between the energy cost of both sessions and the included correlates are presented in 
Table 5.3. They range from 0.169 for the shoulder press (non significant) to 0.642 for weight-training heart rate $(\mathrm{P}<0.001)$.

Table 5.2. The mean, standard deviation $(S D)$ and range (min-max) and relative variance $=($ max$\min )($ (mean 0.01$), \%$ ) of: energy expenditure during the lower and higher intensity weighttraining sessions (EElow and EEhigh, respectively), population characteristics, heart rate, muscular strength and sleeping metabolic rate (SMR) of the included population.

\begin{tabular}{|c|c|c|c|c|}
\hline & mean & $\pm \mathrm{SD}$ & $\min -\max$ & variance $(\%)$ \\
\hline \multicolumn{5}{|l|}{ energy expenditure $\left(\mathrm{kJ} \cdot \mathrm{min}^{-1}\right)$} \\
\hline EElow & 19.8 & \pm 2.1 & $14.8-23.7$ & 45 \\
\hline EEhigh & 22.2 & \pm 2.0 & $17.6-25.6$ & 36 \\
\hline \multicolumn{5}{|l|}{ population characteristics } \\
\hline age $(y)$ & 32.3 & \pm 5.6 & $23-41$ & 56 \\
\hline body mass (kg) & 79.3 & \pm 9.0 & $56.1-103.5$ & 60 \\
\hline height (m) & 1.82 & \pm 0.05 & $1.75-1.97$ & 12 \\
\hline body composition (\%fat) & 17.7 & \pm 5.9 & 7.9- 29.6 & 123 \\
\hline fat-free mass $(\mathrm{kg})$ & 65.0 & \pm 6.8 & $49.6-77.5$ & 43 \\
\hline fat mass $(\mathrm{kg})$ & 14.3 & \pm 5.8 & $5.1-26.3$ & 148 \\
\hline \multicolumn{5}{|l|}{ heart rate (beats $\mathrm{min}^{-1}$ ) } \\
\hline weight training low & 100.7 & \pm 14.4 & $73-130$ & 57 \\
\hline weight training high & 111.2 & \pm 15.5 & $80-142$ & 56 \\
\hline supine rest & 67.13 & \pm 8 & $48-80$ & 48 \\
\hline arm cranking & 110.9 & \pm 16.2 & $84-158$ & 67 \\
\hline cycling & 131.6 & \pm 14.2 & $98-165$ & 51 \\
\hline \multicolumn{5}{|l|}{ remaining variables } \\
\hline leg extension (Nm) & 191.4 & \pm 32.1 & $142-266$ & 65 \\
\hline shoulder press (Nm) & 347.9 & \pm 79.9 & $238-523$ & 82 \\
\hline SMR $\left(k J \cdot \min ^{-1}\right)$ & 4.93 & \pm 0.52 & $3.91-6.34$ & 49 \\
\hline
\end{tabular}

During the lower weight-training intensity the stepwise multiple regression analysis (see Table 5.4) resulted in an $82 \%$ explained inter-subject variance including the variables body mass, heart rate and \%fat (37, 40 and $5 \%$, respectively). At the higher exercise intensity $73 \%$ of the inter-subject variance could be explained by heart rate and body mass ( 41 and $32 \%$ respectively). Figure 5.1 depicts the relationship between the measured and predicted energy expenditure during the lower and higher weighttraining intensity. 
Table 5.3. Pearson Correlation Coefficients between selected potential correlates and energy expenditure during the lower (EElow) and higher (EEhigh) weight-training intensity.

\begin{tabular}{|c|c|c|c|c|}
\hline \multirow[t]{2}{*}{ potential determinants } & \multicolumn{2}{|c|}{ EElow } & \multicolumn{2}{|c|}{ EEhigh } \\
\hline & Cor. Coef. & (P-value) & Cor. Coef. & (P-value) \\
\hline body mass & .605 & $(.001)$ & .555 & $\cdot(.002)$ \\
\hline height & .471 & $(.010)$ & .510 & $(.005)$ \\
\hline$\%$ fat & .185 & $(.337)$ & .262 & $(.170)$ \\
\hline fat-free mass & .521 & $(.004)$ & .409 & $(.027)$ \\
\hline fat mass & .330 & $(.080)$ & .386 & $(.039)$ \\
\hline \multicolumn{5}{|l|}{ heart rate during } \\
\hline weight training & .530 & $(.003)$ & .642 & $(.000)$ \\
\hline supine rest & .363 & $(.053)$ & .413 & $(.026)$ \\
\hline arm cranking & .287 & $(.131)$ & .318 & $(.029)$ \\
\hline cycling & .289 & $(.128)$ & .344 & $(.067)$ \\
\hline \multicolumn{5}{|l|}{ remaining variables } \\
\hline leg extension & .365 & $(.051)$ & .292 & $(.124)$ \\
\hline shoulder press & .307 & $(.105)$ & .169 & $(.381)$ \\
\hline sleeping metabolic rate & .520 & $(.004)$ & .409 & $(.027)$ \\
\hline
\end{tabular}

Table 5.4. Stepwise multiple regression equations on the energy expenditure during the low (EElow) and high (EEhigh) weight training. Correlates included are: body mass, exercise and resting heart rates, \%fat, fat-free mass, fat mass, height, strength (leg extension, shoulder press) and sleeping metabolic rate.

\begin{tabular}{lcccccc}
\hline & constant & body mass & heart rate & $\%$ fat & $\mathbf{R}^{2}$ & F-value \\
EElow & & & & & \\
step 1 & 8.394 & .1433 & & .37 & 15.5 \\
step 2 & -3.239 & .168 & .096 & & .77 & 44.1 \\
Step 3 & -4.727 & .200 & .103 & -0.97 & .83 & 40.1 \\
EEhigh & & & & & & \\
step 1 & 12.877 & & .084 & & .41 & 19.0 \\
step 2 & 2.827 & .126 & .084 & & .73 & 34.6 \\
\hline
\end{tabular}




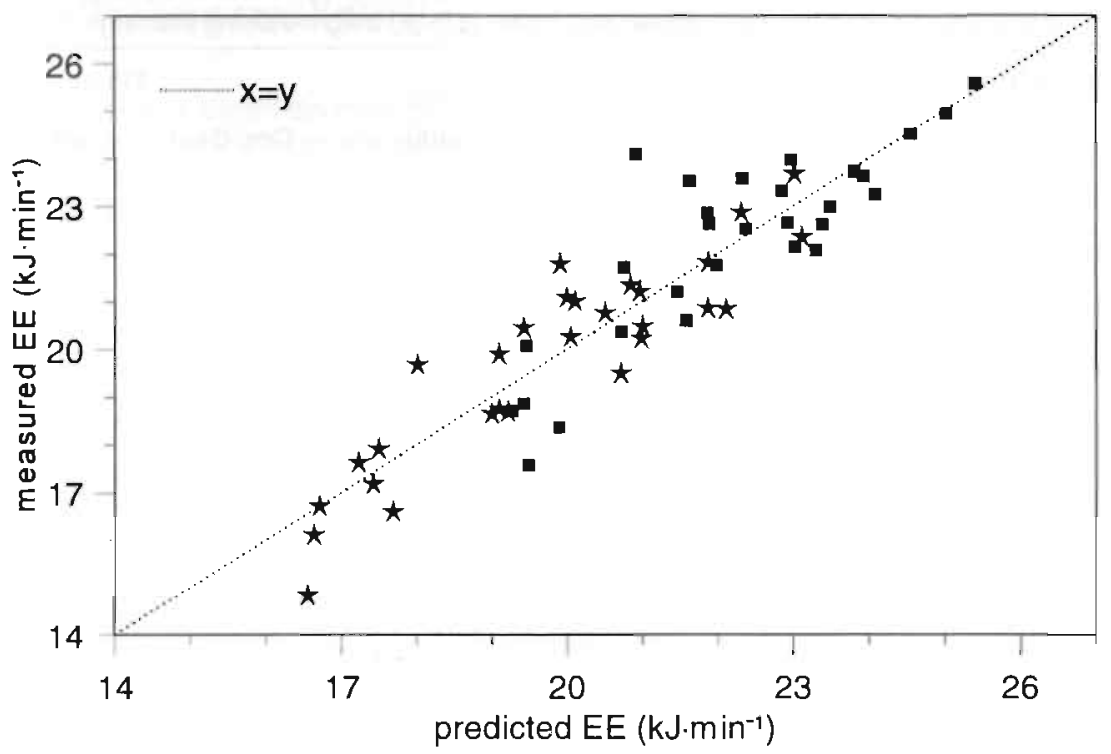

Fig 5.1. Measured energy expenditure (EE) of the lower $(\star$ ) and higher ( $\mathbf{E}$ ) weight-training intensity plotted against the predicted values based on the multiple regression equations derived from the corresponding weighttraining session (see Table 5.4).

The regression equations of EElow and EEhigh over body mass revealed y-intercepts that significantly differed from zero $(\mathrm{P}<0.01$ : Eelow $=8.39+0.14 \times$ body mass, Eehigh $=$ $12.32+0.12 \times$ body mass).

Sleeping metabolic rate, leg extension and the shoulder press data were significantly $(\mathrm{P}<0.001)$ correlated with body mass $(0.72,0.69$ and 0.68 , respectively) and fat-free mass $(0.83,0.77$ and 0.70 , respectively).

Predicting the energy cost of weight-training studies found in the literature (see Table 5.5) based on the regression equations derived from the described protocol resulted in a consistent underestimation of EE (ranging from -8 to $-48 \%$ ).

\section{DISCUSSION}

The aim of the present study was to examine which physiological and/or anthropometrical variables are related to the energy cost of weight-training, besides the already mentioned absolute training load, work/rest-ratio and total number of repetitions. A prerequisite for this type of research is a substantial variation within the dependent as well as independent variables. From Table 5.1 it is apparent that the experimental group met this criterium by demonstrating a wide data range in EE during weight training as well as anthropometrical and physiological variables. Another necessary 
Table 5.5. Data on sexe, percentage exercise time (\%exerc, i.e., work/rest ratio), type of training (WT=weight-training, CWT=circuit weight-training, hydr=hydraulic equipment), duration of the session, body mass (BM), heart rate (HR), body composition (\%fat) and data on measured energy expenditure (actual EE). Based on these data, EE was predicted using the equations as derived from the higher (EQ2 including $H R$ and $B M$ ) and lower weight-training intensity (EQ3 including HR, BM and \%fat; see Table 5.4). The last colum represents the largest relative differences between actual $E E$ and predicted $E E$.

\begin{tabular}{|c|c|c|c|c|c|c|c|c|c|c|c|}
\hline \multirow[b]{2}{*}{ source } & \multicolumn{4}{|c|}{ Training } & \multicolumn{3}{|c|}{ Predictors } & \multicolumn{4}{|c|}{ Results } \\
\hline & sexe & $\begin{array}{l}\text { type of } \\
\text { training }\end{array}$ & $\%$ exer & $\begin{array}{c}\text { duration } \\
\text { (mın) }\end{array}$ & $\begin{array}{c}\text { body mass } \\
(\mathrm{kg})\end{array}$ & $\begin{array}{l}\text { heart rate } \\
\text { (beats.min') }\end{array}$ & $\%$ fat & $\begin{array}{c}\text { actual EE } \\
\left(\mathrm{kJ} \cdot \mathrm{min}^{\prime}\right)\end{array}$ & $\begin{array}{c}E Q 2 \\
\left(k J \cdot \mathrm{min}^{\prime \prime}\right)\end{array}$ & $\begin{array}{c}E Q 3 \\
\left(k J \cdot \mathrm{min}^{-1}\right)\end{array}$ & $\begin{array}{c}\Delta \mathrm{EE} \\
(\%)\end{array}$ \\
\hline 6 excluded subjects & $\mathrm{m}$ & WT & 50 & 42 & 75.7 & 119.1 & 24.4 & 20.6 & 22.6 & 20.6 & $+9.9 \%$ \\
\hline Garbutt $1994(10)$ & $\mathrm{m}$ & CWT & $?$ & 17.8 & 71.4 & 135.0 & & 45.0 & 23.2 & & $-48 \%$ \\
\hline \multirow[t]{2}{*}{ Wilmore 1978 (31) } & $\mathrm{m}$ & CWT & 66 & 22.5 & 77.5 & 134.8 & 14.3 & 37.7 & 24.0 & 23.3 & $-38 \%$ \\
\hline & $f$ & & 66 & 22.5 & 61.0 & 152.3 & 26.4 & 25.5 & 23.4 & 20.6 & $-19 \%$ \\
\hline \multirow[t]{2}{*}{ Hempel 1985 (12) } & $\mathrm{m}$ & CWT & 100 & 21.6 & 72.8 & 148.3 & 11.3 & 32.6 & 24.5 & 24.0 & $-26 \%$ \\
\hline & $f$ & & 100 & 22.0 & 59.7 & 139.9 & 18.7 & 21.5 & 22.2 & 19.8 & $-8 \%$ \\
\hline Hurley 1984 (14) & $\mathrm{m}$ & CWT & 100 & $?$ & 86.1 & 155.0 & 21.0 & 33.0 & 26.8 & 26.4 & $-20 \%$ \\
\hline Katch $1985(16)$ & $\mathrm{m}$ & hydr CWT & $?$ & $?$ & 73.3 & 163.6 & 12.7 & 37.7 & 25.9 & 25.5 & $-32 \%$ \\
\hline Ballor (4) & $\mathrm{m} / \mathrm{l}$ & hydr CWT & 50 & 27 & 73.9 & 152.2 & 14.6 & 40.6 & 25.0 & 24.3 & $-40 \%$ \\
\hline
\end{tabular}


condition is that other variables that could possibly bias the results should be eliminated as much as possible. To exclude inter-subject variation in EE due to differences in the way the weight-training sessions were carried out, a very strict protocol was used. Besides two rehearsal sessions, strict control of technical performance and computerized pacing, EE was always measured at the same time in the morning and expressed as the average of two measurements per intensity. The repeated measurement method was used to minimize the within subject variability due to circadian and daily variation in exercise EE. Williams et al (30) revealed that averaging for two measurements is sufficient to obtain stable data on EE during running, which is an exercise mode that exhibits comparable daily variation as the used weighttraining protocol $( \pm 3 \%)(28)$. A consequence of the standardization is that the protocol shows similarities with a conventional weight-training protocol (e.g., predetermined number of repetitions) as well as a circuit weight-training protocol (i.e., predetermined work/rest ratio) without the accompanying intra- and inter-subject variability in average work performed due to differences in the work/rest-ratio or number of repetitions completed.

The rationale to include variables in the multiple regression analysis differed between the correlates. Body mass and height were included because of biomechanical considerations (i.e., contributing to the absolute workload and the presence of different levers). \%fat provides information on fat-free mass being the metabolic most active mass during exercise. Data on exercise heart rate and muscular strength provide information about the between-subject differences in relative load (i.e., intensity), whereas sleeping metabolic rate is a separate component of gross energy expenditure. Although it is known from the literature that age affects the economy of exercise (21), it was not included as a possible determinant because of the relative small inter-subject variation (i.e., subjects were all young adults).

Correlation analysis revealed that a clear relationship existed between $\mathrm{EE}$ and the anthropometrical variables body mass, height, and fat-free mass. Also weight-training heart rate and sleeping metabolic rate were significantly related to the energy cost of weight training. Most of these variables, however, are interrelated. Therefore, a statistical correlation with EE denotes not necessarily a direct relationship but could also be an indirect relationship through one of the related variables. The stepwise multiple regression analysis revealed that after correcting for inter-subject differences in body mass and weight-training heart rate, approximately $75 \%$ of the inter-subject variance in EE was explained. At the lower weight-training intensity another $6 \%$ could be explained by differences in \%fat. None of the remaining correlates could explain the remaining $\approx 25 \%$ of the inter-subject variability. The contribution of body mass and exercise heart rate to the inter-subject variation is, however, no surprise.

Although all subjects used exactly the same exercise weights while performing the exercises, body mass contributed also to the total external load because $50 \%$ of the exercise time consisted of weight bearing exercises. A significant relationship between body mass and EE was also found in other weight-training studies. In a study of Hempel et al (12) the differences in body mass could even explain the difference in EE between sexes. Wilmore et al (31) also concluded that EE was a direct function of 
the individual's body mass, but differences between sexes appeared to be strictly a function of \% fat. However, both mentioned studies differed from the present study by using less rigid exercise protocols. External load differed not only due to differences in body mass but also because variable training weights and/or numbers of repetitions were used. Therefore, it is likely that body weight affected EE directly through differences in external load but also indirectly through differences in muscular strength which is closely related to body mass (i.e., higher training weights and/or more repetitions). To correct EE for an inter-subject difference in body mass, EE is frequently express relative to kilogram body mass $\left(\mathrm{kJ} \cdot \mathrm{kg}^{-1} \cdot \mathrm{min}^{-1}\right)$. However, if the $y$-intercept significantly differs from zero as in the present study, this method will introduce an error (26) because of a relative overestimate of body mass of the heavy subjects compared with lightweights. Therefore, some caution is recommended in expressing EE relative to body mass.

The relationship between exercise heart rate and $\mathrm{EE}$ is also explainable. In contrast to body mass, heart rate is no determinant (i.e., dependent variable) of EE. Heart rate is continuously adjusted to meet the demand for oxygen and therefore is the result of an increased energy expenditure. Although a change in work performed by the heart will affect $\mathrm{EE}$, this change will be relatively small because myocardial energy expenditure accounts only for 1-2\% to whole body energy expenditure (17).

Contrary to body mass and heart rate, the contribution of \%fat to the inter-subject variation is less explainable. Given the findings that \%fat is negatively correlated with state of training (32) which itself is negatively correlated with the energy cost of exercise $(3,7)$, it would be more likely that \%fat would show a positive relationship with EE. However, after correcting for body mass and heart rate, an increasing \%fat results in a decreasing EE. A plausible explanation for this phenomenon could be a residual difference in metabolic active tissue (i.e., fat-free mass) after correcting for body mass.

Heart rate during rest and submaximal arm cranking and cycling were parameters that were assumed to give some indication on state of training. However, only heart rate during arm cranking was significantly related to EE. This relationship with EE was eliminated after correcting for exercise heart rate.

Since the exercise intensity using an absolute load (e.g., $\mathrm{kg}$ ) is negatively related to maximal strength, stronger subject performed the workout at a lower intensity (\%max effort). Although the energy cost of weight-training exercise revealed to be exponentially related to exercise intensity $(13,15)$, muscular strength showed no relationship with EE. The relationship between muscular strength and body mass, as well as the application of a nonspecific (i.e., isokinetic) method to measure muscular strength could both nullify the effect of strength on EE.

Sleeping metabolic rate was also significantly corrclated with EE, and showed the highest correlation coefficient with EElow. To what extent this relationship is indirectly brought about by body mass or fat-free mass which are both important determinants of sleeping metabolic rate $(1.26)$ remains unclear. The fact that sleeping metabolic rate ranged only $2.4 \mathrm{~kJ} \cdot \mathrm{min}^{-1}$ whereas EE ranges $8.9 \mathrm{~kJ} \cdot \mathrm{min}^{-1}$ indicates that the effect of sleeping metabolic rate on EE was of minor importance. 
As mentioned in the introduction, weight training is a type of exercise that uses a wide variety of exercise protocols (e.g., work/rest-ratio, lower/upper body work-ratio). To illustrate the protocol-specific relationship between the predictors of $\mathrm{EE}$ found in this study (i.e., HR, body mass and \%fat), the regression equations derived from the lower and higher weight-training intensity were used to predict the energy cost of the excluded 6 subjects and of several weight-training workouts described in the literature (see Table 5.5). All studies from the literature used a circuit weight-training protocol (i.e., predetermined exercise time, work/rest ratio but variable weights and number of repetitions). Measured EE was consistently underestimated except in the group of 6 excluded subjects. The latter group was the only group that used the same protocol. The substantial discrepancy between the measured and predicted EE-values illustrates clearly that there are additional predictors of EE during weight training besides heart rate and body mass. Factors like alternation of different muscle groups, the use of variable weights (i.e., closer to maximum weights), shorter duration of the workouts and a larger percentage of exercise time offered the opportunity to increase the intensity of the workout and therefore $\mathrm{EE}$.

In conclusion, more than $70 \%$ of the inter-subject variation in EE during weight training could be explained by differences in body mass and exercise heart rate. To what extent body mass affects EE through the fact that it is part of the external load and/or because it is interrelated with strength, fat-free mass and/or sleeping metabolic rate remains unclear. The multiple regression equation of $\mathrm{EE}$ over the correlates body mass, exercise heart rate and \%fat, however, is protocol specific and only applicable in predicting EE for the protocol described in this study.

\section{REFERENCES}

1 Ainsworth, B. E., W. L. Haskell, A. S. Leon. H. J. Montoye, R. S. Paffenbarger. Compendium of physical activities: classification of energy costs of human physical activities. Med. Sci. Sports Excrc. 25(1):71-80, 1993.

2 American College of Sports Medicine, Guidelines for exercise testing and prescription, 4th ed. Philadelphia: Lea \& Febiger, 1991.

3 Bailey, S. P., R. R. Pate. Feasibility of improving running economy. Sports Med. 12(4):228-236, 1991.

4 Ballor, D. L., M. D. Becque, C. R. Marks, K. L. Nau, V. L. Katch. Physiological response to nine different exercise:rest protocols. Med. Sci. Sports Exerc. 22(1):90-95, 1989.

5 Collins, M. A., K. J. Cureton, D. W. Hill, C. A. Ray. Relationship of heart rate to oxygen uptake during weight lifting exercise. Med. Sci. Sports Exerc. 23(5):636640,1991 .

6 Dale van, D., P. F. M. Schoffelen, F. Hoor ten, W. H. M. Saris. Effects of addition of exercise to energy restriction on 24-hour energy expenditure. sleeping metabolic rate and daily physical activity. Elur. J. Clin. Nutr. 43:441-451, 1989. 
7 Daniels, J. T. A physiologist's view of running economy. Med. Sci. Sports Exerc. 17(3):332-338, 1985.

8 Emons, H. J. G., D. C. Groenenboom, K. R. Westerterp, W. H. M. Saris. Comparison of heart rate monitoring combined with indirect calorimetry and the doubly labelled water () method for the measurement of energy expenditure in children. Eur. J. Appl. Physiol. 65:99-103, 1992.

9 Fuller, N. J., S. A. Jebb, M. A. Laskey, W. A. Coward, M. Elia. Four-component model for the assessment of body composition in humans: comparison with alternative methods, and evaluation of the density and hydration of fat -free mass. Clin. Sci. 82:687-693, 1992.

10 Garbutt, G., M. G. Boocock, J. D. G. Troup. Physiological and spinal responses to circuit weight-training. Ergonomics 37(1):117-125, 1994.

11 Heini, A., Y. Schutz, E. Diaz, A. M. Prentice, R. G. Whitehead, E. Jequier. Freeliving energy expenditure measured by two independent techniques in pregnant and nonpregnant Gambian women. Am. J. Physiol. 261:E9-E17, 1991.

12 Hempel, L. S., C. L. Wells. Cardiorespiratory cost of the Nautilus express circuit. Phys. Sportsmed. 13(4):82-97, 1985.

13 Hunter, G., L. Blackman, L. Dunnam, G. Flemming. Bench press metabolic rate as a function of exercise intensity. J. Appl. Sport Sci. Res. 2(1):1-6, 1988.

14 Hurley, B. F., D. R. Seals, A. A. Ehsani, et al. Effects of high-intensity strength training on cardiovascular function. Med. Sci. Sports Exerc. 16(5):483-488, 1984.

$15 \mathrm{Kalb}$, J. S., G. R. Hunter. Weight training economy as a function of intensity of the squat and overhead press exercise. J. Sports Med. Phys. Fitness 31:154-160, 1991.

16 Katch, F. I., P. S. Freedson, C. A. Jones. Evaluation of acute cardiorespiratory responses to hydraulic resistance exercise. Med. Sci. Sports Excrc. 17:168-173, 1985.

17 Kitamura, K., C. R. Jorgensen, F. L. Gobel, H. L. Taylor, Y. Wang. Hemodynamic correlates of myocardial oxygen consumption during upright exercise. J. Appl. Physiol. 32(4):516-522, 1972.

$18 \mathrm{Li}$, R., P. Deurenberg, J. G. A. J. Hautvast. A critical evaluation of heart rate monitoring to assess energy expenditure in individuals. Am. J. Clin. Nutr. 58:602$607,1993$.

19 Lothian, F., M. R. Farrally. A comparison of methods for estimating oxygen uptake during intermittent exercise. Journal of Sports Sciences 13:491-497, 1995.

20 Maas, S., M. L. J. Kok, H. G. Westra, H. C. G. Kemper. The validity of the use of heart rate in estimating oxygen consumption in static and in combined static/ dynamic exercise. Ergonomics 32(2):141-148, 1989.

21 Morgan, D. W., P. E. Martin, G. S. Krahenbuhl. Factors affecting running economy. Sports Med. 7:310-330, 1989.

22 Murgatroyd, P. R., P. S. Shetty, A. M. Prentice. Techniques for the measurement of human energy expenditure: a practical guide. International Journal of Obesity 17:549-568, 1993. 
23 Oja, P., J. Ilmarinen, V. Louhevaara. Heart rate as an estimator of oxygen consumption during manual postal delivery. Scand. J. Work Environ. Health 8:29$36,1982$.

24 Parker, S. B., B. F. Hurley, P. D. Hanlon. P. Vaccaro. Failure of target heart rate to accurately monitor intensity during aerobic dance. Med. Sci. Sports Exc. 21(2):230-234, 1989.

25 Pendergast, D. R. Cardiovascular, respiratory, and metabolic responses to upper body exercise. Med. Sci. Sports Exerc. 21(5):s121-s125, 1989.

26 Ravussin, E., C. Bogardus. Relationship of genetics, age, and physical fitness to daily energy expenditure and fuel utilization. Am. J. Clin. Nutr. 49:968-975, 1989.

27 Sawaka, M. N., D. S. Miles, J. S. Petrofsky, S. W. Wilde, R. M. Glaser, Physiology of upper body exercise, in Exercise and Sports Sciences Reviews, vol. 14, Pandolf, K. B., Ed. New York: Macmillan, 1986, pp. 175-211.

28 Van Etten, L. M. L. A., K. R. Westerterp, F. T. J. Verstappen. Daily variation in energy expenditure during weight-training versus continuous arm cranking and cycling. Int. J. Sports Med. 17(7):497-503, 1996.

29 Weir de, J. B. New methods for calculating metabolic rate with special reference to protein metabolism. J. Physiol. 109:1-9, 1949.

30 Williams, T. J., G. S. Krahenbuhl, D. W. Morgan. Daily variation in running economy of moderately trained male runners. Med. Sci. Sports Exerc. 23(8):944$948,1991$.

31 Wilmore, J., R. Parr, P. Ward, P. Vodak, T. Barstow. Energy cost of circuit weight training. Med. Sci. Sports Exerc. 10(2):75-78, 1978.

32 Wilmore, J. H. Variations in physical activity habits and body composition. Int. J. Obesity 19(sup 4):S107-S112, 1995. 


\section{Chapter 6}

\section{Effect of 18-weeks of weight training on exercise economy}

Ludo M.L.A. van Etten, Frans T.J. Verstappen*, Klaas R. Westerterp, Bart J.B. Boon and Wim H.M. Saris.

Department of Human Biology and "Department of Movement Sciences, Maastricht University, Maastricht, The Netherlands

Submitted

\section{ABSTRACT}

To study the effect of training on energy expenditure during exercise (expressed in $\left.\mathrm{kJ} \cdot \mathrm{min}^{-1}\right), 18$ sedentary males followed an 18 week weight-training program whereas six subjects served as inactive controls. Pre- and post-intervention exercise economy was measured during four sessions $(2 x$ arm cranking plus lower intensity weight training, $2 x$ cycling plus higher intensity weight training). In the trained group initial energy expenditure (EE) during lower intensity weight training $\left(21.5 \pm 2.4 \mathrm{~kJ} \cdot \mathrm{min}^{-1}\right)$, higher intensity weight training $\left(24.2 \pm 2.8 \mathrm{~kJ} \cdot \mathrm{min}^{-1}\right)$, arm cranking $(24.6 \pm 2.0$ $\left.\mathrm{kJ} \cdot \mathrm{min}^{-1}\right)$ and cycling $\left(42.7 \pm 5.9 \mathrm{~kJ} \cdot \mathrm{min}^{-1}\right)$ decreased with $9.2,9.9,8.0$ and $6.9 \%$, respectively. Initial heart rate (HR: $118.8 \pm 14.5,128.5 \pm 15.3,131.3 \pm 16.5$ and 145.2 \pm 15.7 beats $\left.\mathrm{min}^{-1}\right)$ decreased with $14.5,13.1,13.7$ and $9.7 \%$, respectively. The increases in isokinetic strength (9.5\%) and VO2max (17.5\%) were not correlated with the improved exercise economy. The control group showed no change in exercise economy and physical performance. Therefore, it was concluded that 18 weeks of weight training decreased $E E$ and HR during intermittent weight-training exercises, as well as continuous arm cranking and cycling. 


\section{INTRODUCTION}

Although with opposite interests, the energy cost of exercise is a research topic for sports as well as health scientists. The sports scientist tries to find methods to decrease energy expenditure during endurance exercise since the availability of energy is an important determinant of performance during prolonged exercise. The health scientist, on the other hand, searches for the most feasible and effective mode of exercise to increase physical activity and daily energy expenditure with the intention to improve physical fitness and body composition.

Maximal aerobic capacity (VO2max) as well as exercise economy (i.e. oxygen demand of a standard submaximal exercise) are both important determinants of endurance performance. However, no consensus exists regarding the effect of training status on exercise economy. Case reports on elite runners revealed an improvement in exercise economy from off-season to competitive season $(7,8)$. Several cross-sectional as well as longitudinal studies on endurance exercise also revealed that a higher training status improves exercise economy (i.e. decreases VO2submix) but other investigators found no effect or even a reduced excrcise economy (i.e. increased VO2submax $)(12,23,24)$. According to Morgan et al (23), part of this inconsistency was due to the type and intensity of the training program or to limitations in experimental design (e.g sample size, intra individual variation, training accommodation). However, if the energy cost of physical exercise can be altered by training, studies on the effect of exercise on daily energy expenditure should take this training induced change in exercise economy into account when estimating the energy cost of the added activity.

Health benefits from changing a sedentary into a more active lifestyle by physica] training are not restricted to endurance training but can also be realized by choosing an intermittent or a combined mode of excrcise (e.g basketball, aerobic dance, weight training). Although weight-training interventions are frequently used in research on health and fitness determinants (including overweight), until now, no research was done on the effect of this intermittent type of training on exercise economy.

This study examined the effect of an 18 wk weight-training program (two workouts. $\mathrm{wk}^{-1}, \mathrm{n}=18$ ) on exercise economy during intermittent weight training as well as continuous arm cranking and cycling. Previous studies defined exercise economy as the rate of oxygen consumption at a given submaximal workload ( $\mathrm{VO}$ 2submax) (4). Since changes in substrate utilization aflect the oxygen demand of energy production, this study expresses exercise economy in $\mathrm{kJ} \cdot \mathrm{min}^{-1}$.

\section{METHODS}

The overall design of the study is presented in figure 6.1.

Subjects. Twenty-four healthy sedentary males were selected to participate in the study. None of the subjects had engaged in a regular exercise program for at least two 


\begin{tabular}{|c|c|c|c|c|c|c|c|c|c|c|c|c|c|c|c|c|c|c|c|c|c|c|c|}
\hline subjects & week no: & -2 & -1 & 1 & 2 & 3 & 4 & 5 & 6 & 7 & 8 & 9 & 10 & 11 & 12 & 13 & 14 & 15 & 16 & 17 & 18 & 19 & 20 \\
\hline \multirow[t]{4}{*}{ EXER } & workout fitness club & & & & & & & & & & & & & & & & & & & & & & \\
\hline & workout university & & & 0 & & 0 & & & & & & & & & & & & & & & & O & 0 \\
\hline & HR workout club & & & & & & & & & & & & & & & & & & & & & & \\
\hline & body composition & $x$ & & & & & & & & & & & & & & & & & & $\mathrm{x}$ & & & \\
\hline \multirow[t]{2}{*}{ CONT } & workout university & & & 0 & & 0 & & & & & & & & & & & & & & & & 0 & 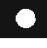 \\
\hline & body composition & $x$ & & & & & & & & & & & & & & & & & & $x$ & & & \\
\hline
\end{tabular}

Fig. 6.1. Overall design. The actual training program consisted of 18 weeks of weight training( $)$. The first three weeks were pertormed at the university to determine EE during standardized training sessions. Week 19 and 20 were not considered as part of the training program but were used to check if the energy expenditure-heart rate relationship was changed. During week 1 and 19 an incremental cycling test was performed (a) and during week 3 and 20 both strength tests were administered (D). 
years prior to the study. Detailed information over the purpose and all methods used in the study was provided and written consent was obtained. Eighteen subjects participated in the exercise group (EXER) and six subjects served as inactive controls (CONT).

Physical characteristics (see Table 6.1). Anthropometrical measurements were taken a morning after a controlled overnight stay at the laboratory. After voiding body mass was measured to an accuracy of $5 \mathrm{~g}$ on an electronic scale (Mettler, E1200). Body volume was determined using the hydrostatic weighing technique. Residual lung volume was measured simultaneously using the helium dilution technique (Volugraph 200, Mijnhart). Total body water (TBW) was measured using deuterium $\left({ }^{2} \mathrm{H}_{2} \mathrm{O}\right.$ ) dilution according to the Maastricht protocol (34). Body composition was assessed using the three-component model of Siri that combines body density with TBW (14).

Table 6.1. Initial characteristics (mean $\pm S D$ ) and changes $(\Delta)$ in body composition in the exercise-group (EXER, $n=18$ ) and the control group (CONT, $n=6)$.

\begin{tabular}{|c|c|c|c|c|}
\hline & \multicolumn{2}{|c|}{ EXER } & \multicolumn{2}{|c|}{ CONT } \\
\hline & initial & $\Delta$ & initial & $\Delta$ \\
\hline age $(y)$ & $33 \pm 6$ & & $36 \pm 7$ & \\
\hline height (m) & $1.82 \pm 0.07$ & & $1.80 \pm 0.05$ & \\
\hline body mass (kg) & $78.8 \pm 9.7$ & $0.1 \pm 1.5$ & $77.0 \pm 11.8$ & $-1.0 \pm 1.4$ \\
\hline fat mass $(\mathrm{kg})$ & $19.1 \pm 4.7$ & $-2.0 \pm 1.8^{* *}$ & $18.0 \pm 6.6$ & $-1.6 \pm 0.9^{* *}$ \\
\hline fat free mass $(\mathrm{kg})$ & $59.7 \pm 6.6$ & $2.1 \pm 1.2^{* *}$ & $59.5 \pm 7.5$ & $0.6 \pm 1.9^{\prime \prime}$ \\
\hline fat (\%) & $24 \pm 4$ & $-2.6 \pm 2.0^{* *}$ & $23 \pm 7$ & $-1.9 \pm 1.4^{*}$ \\
\hline
\end{tabular}

Pre- and post-intervention differences ${ }^{P} P<0.05, " \mathrm{P}<0.001$

Significantly different between EXER and CONT 'P $<0.05$

The intervention (training program). The subjects trained two times a week on non-consecutive days for 18 weeks. The workouts at the fitness club consisted of 10 exercises using weight stack machines (Sportesse, Germany) and free weights. Initial training weights were based on the subject's weight and baseline performance. Three sets of 15 repetitions were performed on the following exercises: bench press, flys, squat, leg curl, leg extension, seated rowing, lat pull down, dumbbell curl, triceps push down and the sit ups. Throughout the training program, training weight was adjusted as strength levels increased provided that the subject was able to perform three sets of 15 repetitions. Additionally, each workout included a 10 min warming-up and coolingdown of cycling on a self selected intensity. All workouts were supervised by a fitness instructor.

Exercise economy. To determine exercise economy, the first six weight-training sessions were performed in the laboratory. Each session included a weight-rraining 
phase, a warming-up phase, and a cooling-down phase. The weight-training phase consisted of 3 sets of 15 repetitions of the following exercises: bench press, lunges, dumbell pull, squat, and the overhead lats pulley ( 3 upper body and 2 lower body excrcises) using standard weights. This sequence of five exercises was completed twice to increase the volume of the session without increasing the number of different exercises (see table 6.2). Total duration of the weight-training phase was 42 minutes. Since a single repetition of the lunges consisted of an alternating left and right step forward, total duration of this exercise was approximately twice the time used by other exercises. Hence, exercise time was equally split up between the upper and lower part of the body. The work to rest ratio was $1: 1$. A computer-generated acoustic signal dictated the pace during exercise and the duration of the resting intervals. Variability in technical performance was minimized by using strict technique demands. The initial training load during the weight-training phase of session 1 to 4 (lower WT-intensity) was increased by raising training weights by approximately $50 \%$ during session 5 and 6 (higher WT-intensity). As is customary, subjects also pertormed a warming-up and cooling-down (both $10 \mathrm{~min}$ ). During the first four sessions arm ergometry (i.e. arm cranking; 48 watt/ 60 rpm, Ergostat Universal, Switzerland) was used, whereas session five and six used leg ergometry (i.e. cycling; 150 watt/ $75 \mathrm{rpm}$, Jaeger ER800) as warming-up and cooling-down exercise. For five subjects (EXER: $n=3$, CONT: $n=2$ ), the workload had to adjusted because they were not able to carry out the session using the standard load (arm cranking 42 watt, cycling 100 watt, no increase in training load for the leg exercises).

Table 6.2. Protocol and sequence of the six training sessions. The load and pedaling rate during armcranking and cycling were 48 [42] watt at $60 \mathrm{rpm}$, and 150 [100] watt at $75 \mathrm{rpm}$, respectively. The duration of the weight-training phase was 42 minutes. Training weights are expressed in $\mathrm{kg}$. For 5 subjects workload had to be adjusted (adjusted load between brackets). Session 1 and 2 were used for accommodation. During session 3-6 (L1-H2) cardiorespiratory data were collected.

\begin{tabular}{|c|c|c|c|c|c|c|c|}
\hline & Exercise & $\begin{array}{c}\text { session } \\
1 \\
\end{array}$ & $\begin{array}{c}\text { session } \\
2 \\
\end{array}$ & $\begin{array}{c}\text { session } \\
3(\text { L1) }\end{array}$ & $\begin{array}{c}\text { session } \\
4(L 2)\end{array}$ & $\begin{array}{c}\text { session } \\
5(\mathrm{H} 1)\end{array}$ & $\begin{array}{c}\text { session } \\
6(\mathrm{H} 2)\end{array}$ \\
\hline \multicolumn{2}{|c|}{ warming up (10 min) } & cranking & cranking & cranking & cranking & cycling & cycling \\
\hline & bench press (kg) & 10 & 10 & 10 & 10 & 15 & 15 \\
\hline & lunges (kg) & 25 & 25 & 25 & 25 & 35 [25] & 35 [25] \\
\hline \multirow[t]{3}{*}{$2 x$} & dumbell pull $(\mathrm{kg})$ & $2 \times 4$ & $2 \times 4$ & $2 \times 4$ & $2 \times 4$ & $2 \times 5^{1 / 2}$ & $2 \times 5 \frac{1}{2}$ \\
\hline & squat (kg) & 30 & 30 & 30 & 30 & $45[30]$ & $45[30]$ \\
\hline & lats pulley (kg) & $14 \frac{1}{4}$ & $141 / 4$ & $14 \frac{1}{4}$ & $141 / 4$ & 20 & 20 \\
\hline \multicolumn{2}{|c|}{ cooling down (10 min) } & cranking & cranking & cranking & cranking & cycling & cycling \\
\hline
\end{tabular}

Session measurements. From the total of 6 training sessions, the first two sessions were used to accommodate the subjects to the laboratory setting and computerized pacing. No measurements were carried out and the subjects had full freedom of movement since they were not connected to the gas analyzer. The remaining four measurement sessions consisted of two sessions performed at the lower WT-intensity 
(session L1, L2) and two sessions performed at the higher WT-intensity (session $\mathrm{H1}$, H2). The balanced increase in training load was necessary because most of the subjects had no previous experience with weight training. To create similar starting conditions subjects were asked to sit as relaxed as possible for five minutes followed by another ten minutes of supine rest prior to every session. Resting energy expenditure and heart rate, were defined as the lowest $2 \frac{1}{2} \mathrm{~min}$-value over the entire $15 \mathrm{~min}$ pre-session period. To determine recovery energy expenditure this procedure was repeated immediately following the cooling down.

All sessions were conducted at the same time in the morning to minimize the residual effects of physical activity, food intake and circadian variation, on energy expenditure. Furthermore, subjects were asked to refrain from any exercise or strenuous physical activity 36 hours before each session and to eat similar breakfasts. Data on gasexchange and heart rate were collected during the entire experiment (including the resting periods).

Gas-exchange was continuously measured by open circuit spirometry using an automated system (Oxycon Beta, Mijnhardt). Respiratory parameters were calculated over an average of 8 breath cycles and recorded every 10 seconds $\left(\mathrm{ml} \cdot \mathrm{min}^{-1}\right)$. Gas analyzers were automatically calibrated before each experiment with standard gas mixtures. Gas-exchange was converted to energy expenditure using the Weir formula (33).

Heart rate (HR) was continuously monitored using a Polar Sport Tester PE3000 and reported over 10 seconds intervals (beats $\mathrm{min}^{-1}$ ).

After eighteen weeks exercise economy was again determined using the same protocol and loads with one difference namely only one session was used to accommodate (i.e, a total of five sessions).

Maximal aerobic power (VO2max). A stepwise incremental cycling test was performed to determine VO2max. The test started with 4 min cycling at $50 \mathrm{~W}$. Subsequently, load was increased every three minutes with respectively $50 \mathrm{~W}$ if $\mathrm{HR}<160$ beats $\mathrm{min}^{-1}$ and $25 \mathrm{~W}$ if $\mathrm{HR} \geq 160$ beats $\mathrm{min}^{-1}$, until exhaustion.

Muscular strength. To get information about muscular strength in the upper thigh and shoulder/arm an isokinetic dynamometer (Cybex II, Lumex Inc., New York) was used: knee extension at $2.094 \mathrm{rad} \cdot \mathrm{s}^{-1}\left(=120^{\circ} \cdot \mathrm{s}^{-1}\right)$; shoulder/arm during a bench press movement at $1.047 \mathrm{rad} \cdot \mathrm{s}^{-1}\left(60^{\circ} \cdot \mathrm{s}^{-1}\right)$. Subjects performed one set of five repetitions at each velocity from which the peak torque was noted as the parameter for maximal strength.

Workout heart rate. To estimate the weight-training intensity during the workouts at the fitness club, heart rate was measured during week 8-9 and week 17-18 (Polar Sport Tester PE3000).

Statistical analysis. Paired $t$-tests were used to analyze differences between test-retest sessions and pre-. versus post-intervention measurements. To analyze differences 
between the exercise and control group, $t$-tests for independent samples were used. Pearson correlation coefficients and linear multiple regression analyses were used to examine relationships between variables. Statistical significance was set at $\mathrm{P}<0.05$.

\section{RESULTS}

Physical characteristics (see Table 6.1). After 18 weeks both the EXER and CONT group had lost fat mass $(2.0 \pm 1.8 \mathrm{~kg} ; \mathrm{P}<0.001$ and $1.6 \pm 0.9 \mathrm{~kg} ; \mathrm{P}<0.05$, respectively), whereas fat-free mass increased only in EXER $(2.1 \pm 1.2 \mathrm{~kg} ; \mathrm{P}<0.001$ and $0.6 \pm 1.9 \mathrm{~kg}$; NS, respectively). The combined changes in fat mass and fat-free mass resulted in an unaltered body mass $(0.1 \pm 1.5 \mathrm{~kg}$; NS and $-1.0 \pm 1.4 \mathrm{~kg}$; NS, respectively) but a significant decrease in percentage fat of $-2.6 \pm 2.0 \% ; \mathrm{P}<0.001$ and $-1.9 \pm 1.4 \% ; \mathrm{P}<0.05$, respectively.

Single session weight training energy expenditure (EE) and heart rate (HR). In EXER, pre-intervention EE during the two lower intensity test-retest weight-training phases was $22.1 \pm 2.3$ and $21.0 \pm 2.6 \mathrm{~kJ} \cdot \mathrm{min}^{-1}$. For the higher intensity test-retest weighttraining phases EE was $24.6 \pm 2.5$ and $23.9 \pm 2.9 \mathrm{~kJ} \cdot \mathrm{min}^{-1}$, respectively (see Fig. 6.2). Corresponding $\mathrm{HR}$ was $120 \pm 15,117 \pm 15,128 \pm 16$ and $128 \pm 16$ beats $\cdot \mathrm{min}^{-1}$, respec-

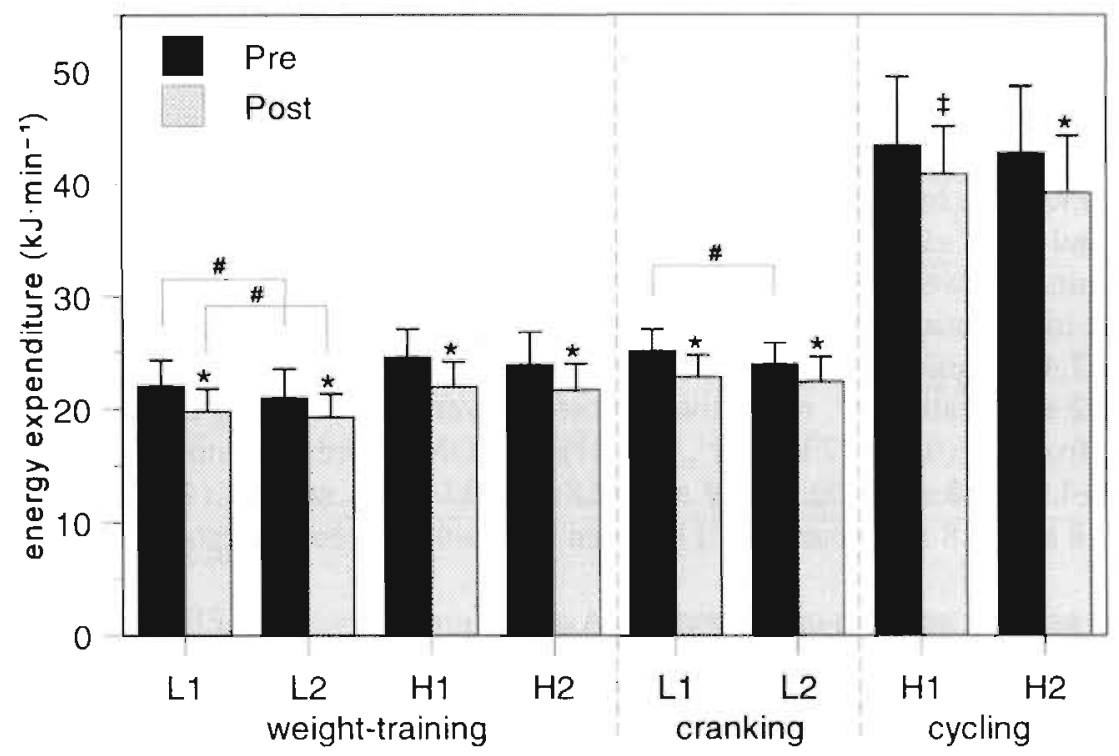

Fig. 6.2. Pre-and post-intervention energy expenditure in the exercise group during the weight-training phase and the warming-up phase (cranking or cycling) of the four computer paced sessions (lower intensity L1, L2 and higher intensity $\mathrm{H1}, \mathrm{H2}$, respectively; pre- and post intervention differences: ${ }^{i} P<0.05,{ }^{\prime} P<0.001$; test-refes! differences ' $P<0.01$ ). 


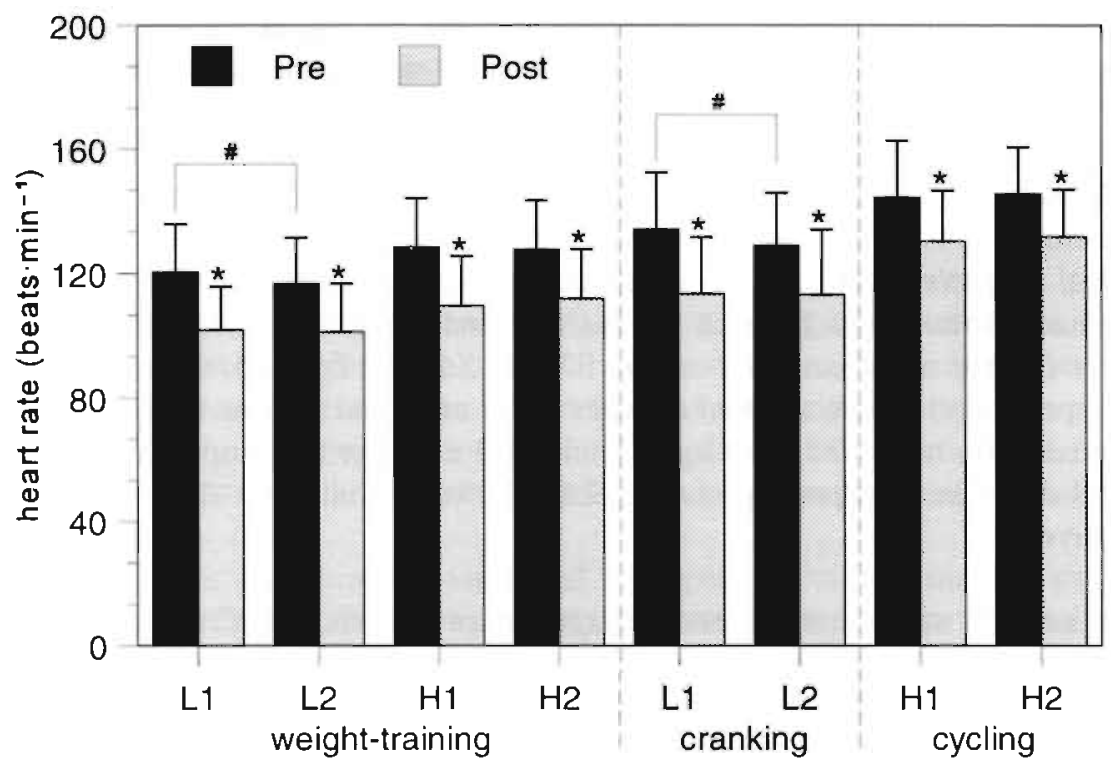

Fig. 6.3. Pre- and post-intervention heart rate in the exercise group during the weight-training phase and the warming-up phase (cranking or cycling) of the four computer paced sessions (lower intensity L1, L2 and higher intensity $\mathrm{H1}, \mathrm{H2}$, respectively; $p$ re- and post intervention differences ' $P<0.001$; test-retest differences ' $P<0.05$ ).

vely (see Fig. 6.3). Paired $t$-tests revealed a significantly lower EE and HR during the repeated lower intensity session ( $\mathrm{L} 2,-1.1 \pm 0.9 \mathrm{~kJ} \cdot \mathrm{min}^{-1}, \mathrm{P}<0.001$ and $-3.6 \pm 4$ beats. $\left.\min ^{-1}, \mathrm{P}<0.05\right)$. In CONT there were no differences in EE $(20.7 \pm 3.7,20.2 \pm 3.4,22.5$ \pm 2.2 and $\left.22.5 \pm 2.1 \mathrm{~kJ} \cdot \mathrm{min}^{-1}\right)$ nor $\mathrm{HR}(114 \pm 12,114 \pm 15,123 \pm 12$ and $117 \pm 11$ beats $\left.\cdot \mathrm{min}^{-1}\right)$ between the pre-intervention test-retest sessions.

Post-intervention EE in EXER was $19.8 \pm 2.0$ and $19.3 \pm 2.1 \mathrm{~kJ} \cdot \mathrm{min}^{-1}, 22.0 \pm 2.3$ and $21.7 \pm 2.4 \mathrm{~kJ} \cdot \mathrm{min}^{-1}$, respectively. Corresponding HR was $102 \pm 14,101 \pm 15,110 \pm 16$ and $112 \pm 16$ beats $\min ^{-1}$, respectively. Post-intervention EE during $\mathrm{L} 2$ differed significantly from $\mathrm{Ll}\left(0.5 \pm 0.7 \mathrm{~kJ} \cdot \mathrm{min}^{-1}, \mathrm{P}<0.01\right)$. In CONT there were no differences in EE $\left(20.8 \pm 4.2,20.2 \pm 2.6,22.9 \pm 2.9\right.$ and $\left.22.8 \pm 2.5 \mathrm{~kJ} \cdot \mathrm{min}^{-1}\right)$ nor $\mathrm{HR}(111 \pm 15,107 \pm 12$, $120 \pm 18$ and $118 \pm 10$ beats $\cdot \mathrm{min}^{-1}$ ) between the post-intervention test-retest sessions.

Single session warming-up EE and HR. Average pre-intervention EE over the last five minutes of the arm cranking was $25.2 \pm 2.0,24.0 \pm 1.9 \mathrm{~kJ} \cdot \mathrm{min}^{-1}$ and for cycling 43.5 \pm 6.1 and $42.8 \pm 5.9 \mathrm{~kJ} \cdot \mathrm{min}^{-1}$ (see Fig. 6.2). Corresponding HR was $134 \pm 18,129 \pm 17$, $144 \pm 18$ and $146 \pm 15$ beats min $^{-1}$, respectively (see Fig. 6.3). Paired $t$-tests revealed a significantly lower EE and HR during arm-cranking of session L2 $\left(1.2 \pm 1.7 \mathrm{~kJ} \cdot \mathrm{min}^{-1}\right.$, $\mathrm{P}<0.01$ and $5.9 \pm 12$ beats $\mathrm{min}^{-1}, \mathrm{P}<0.05$ ). In CONT there were no differences in EE $\left(22.8 \pm 2.9 .22 .7 \pm 2.7,39.1 \pm 4.6\right.$ and $\left.38.3 \pm 4.1 \mathrm{~kJ} \cdot \mathrm{min}^{-1}\right)$ nor $\operatorname{HR}(121.0 \pm 12,122 \pm 12$, $140 \pm 18$ and $137 \pm 13$ beats $\left.\cdot \mathrm{min}^{-1}\right)$ between the pre-intervention test-retest sessions. 
Post-intervention EE (arm cranking: $22.8 \pm 1.9,22.4 \pm 2.2$ and cycling: $40.9 \pm 4.2$ and $\left.39.3 \pm 5.1 \mathrm{~kJ} \cdot \mathrm{min}^{-1}\right)$ and $\mathrm{HR}\left(114 \pm 18,113 \pm 20,130 \pm 16\right.$ and $132 \pm 15$ beats $\mathrm{min}^{-1}$, respectively) did not differ between the repeated sessions. In CONT there were also no differences in $\mathrm{EE}\left(21.8 \pm 1.0,21.9 \pm 0.7,39.4 \pm 4.6\right.$ and $\left.40.0 \pm 5.6 \mathrm{~kJ} \cdot \mathrm{min}^{-1}\right)$ nor in HR $\left(118 \pm 14,115 \pm 10,135 \pm 1.5\right.$ and $138 \pm 14$ beats. min $\left.^{-1}\right)$ between the post-intervention test-retest warming-up.

Changes in exercise economy. After 18 wk of training average EE during the weighttraining phase of session $\mathrm{L} 1, \mathrm{~L} 2, \mathrm{H} 1$ and $\mathrm{H} 2$ decreased by $2.3 \pm 1.2,1.7 \pm 1.5,2.6 \pm 1.3$ and $2.3 \pm 1.5 \mathrm{~kJ} \cdot \mathrm{min}^{-1}$, respectively $(\mathrm{P}<0.001$, see Fig. 6.2). Average HR decreased by $19 \pm 8,16 \pm 6,19 \pm 8$ and $16 \pm 8$ beats $\mathrm{min}^{-1}$, respectively $(\mathrm{P}<0.001)$. Average EE during the warming-up decreased by $2.3 \pm 2.1,1.6 \pm 1.9,2.6 \pm 4.3$ and $3.5 \pm 3.3 \mathrm{~kJ} \cdot \mathrm{min}^{-1}$ $(\mathrm{P}<0.05)$ and average HR decreased by $21 \pm 11,16 \pm 8,14 \pm 10$ and $14 \pm 9$ beats. $\mathrm{min}^{-1}$ $(\mathrm{P}<0.001)$, respectively.

Table 6.3. Initial values and changes ( $\Delta$ ) in energy expenditure (EE), heart rate (HR), minute ventilation (Ve), breathing frequency (Bf), and tidal volume (TV) after 18 weeks of weight training. Data represent the average of sessions $L 1$ and $L 2(L 1 / 2)$ and sessions $H 1$ and $H 2(H 1 / 2)$.

\begin{tabular}{|c|c|c|c|c|}
\hline weight training & $\lfloor 1 / 2$ & $\Delta \mathrm{L} 1 / 2$ & $H 1 / 2$ & $\Delta \mathrm{H} 1 / 2$ \\
\hline$E E\left(k J \cdot \mathrm{min}^{-1}\right)$ & $21.5 \pm 2.4$ & $-2.0 \pm 1.3^{* *}$ & $24.2 \pm 2.8$ & $-2.4 \pm 1.3^{* *}$ \\
\hline $\mathrm{HR}$ (beats $\mathrm{min}^{-1}$ ) & $118.8 \pm 14.5$ & $-17.3 \pm 6.1^{* *}$ & $128.5 \pm 15.3$ & $-16.9 \pm 7.0^{* *}$ \\
\hline $\operatorname{Ve}\left(1 \cdot \mathrm{min}^{-5}\right)$ & $34.8 \pm 4.1$ & $-4.3 \pm 2.6^{* *}$ & $40.0 \pm 5.3$ & $-4.5 \pm 3.1^{\text {** }}$ \\
\hline $\mathrm{Bf}$ (breath. $\left.\min ^{-1}\right)$ & $24.6 \pm 1.9$ & $-0.8 \pm 1.2^{*}$ & $26.4 \pm 2.5$ & $-1.4 \pm 1.2^{\star *}$ \\
\hline TV (I) & $1.43 \pm 0.15$ & $-0.12 \pm 0.08^{* *}$ & $1.54 \pm 0.17$ & $-0.09 \pm 0.08^{* *}$ \\
\hline warming-up & arm cranking & $\Delta$ & cycling & $\Delta$ \\
\hline $\mathrm{EE}\left(\mathrm{kJ} \cdot \mathrm{min}^{-1}\right)$ & $24.6 \pm 1.7$ & $-2.0 \pm 1.8^{* *}$ & $42.7 \pm 5.9$ & $-3.0 \pm 2.5^{\star *}$ \\
\hline $\mathrm{HR}$ (beats. $\min ^{-1}$ ) & $131.3 \pm 16.5$ & $-18.0 \pm 7.5^{\star \star *}$ & $145.2 \pm 15.7$ & $-14.2 \pm 7.1^{\star *}$ \\
\hline$V e\left(I \cdot \mathrm{min}^{-1}\right)$ & $41.4 \pm 3.9$ & $-4.5 \pm 3.0^{* *}$ & $58.8 \pm 10.6$ & $-5.2 \pm 5.4^{* *}$ \\
\hline Bf (breath $\min ^{-1}$ ) & $26.6 \pm 4.0$ & $-0.8 \pm 2.4$ & $26.6 \pm 4.8$ & $-1.1 \pm 2.1^{*}$ \\
\hline TV (1) & $1.58 \pm 0.18$ & $-0.11 \pm 0.12^{* *}$ & $2.24 \pm 0.29$ & $-0.09 \pm 0.14^{*}$ \\
\hline
\end{tabular}

" $P<0.05,{ }^{* *} P<0.001$

To obtain more stable economy values (35) and to improve readability, values are presented as the average of the repeated lower (L1/2) or higher $(\mathrm{H} 1 / 2)$ WT-intensity sessions, arm cranking and cycling exercise (see Table 6.3). The average relative decrease $(\%)$ in EE during the lower and higher intensity weight training, arm cranking and cycling was $9.2,9.9,8.0$ and $6.9 \%$, whereas HR decreased by $14.5,13.1$, 13.7 and $9.7 \%$, respectively. The control group showed no change in absolute as well 


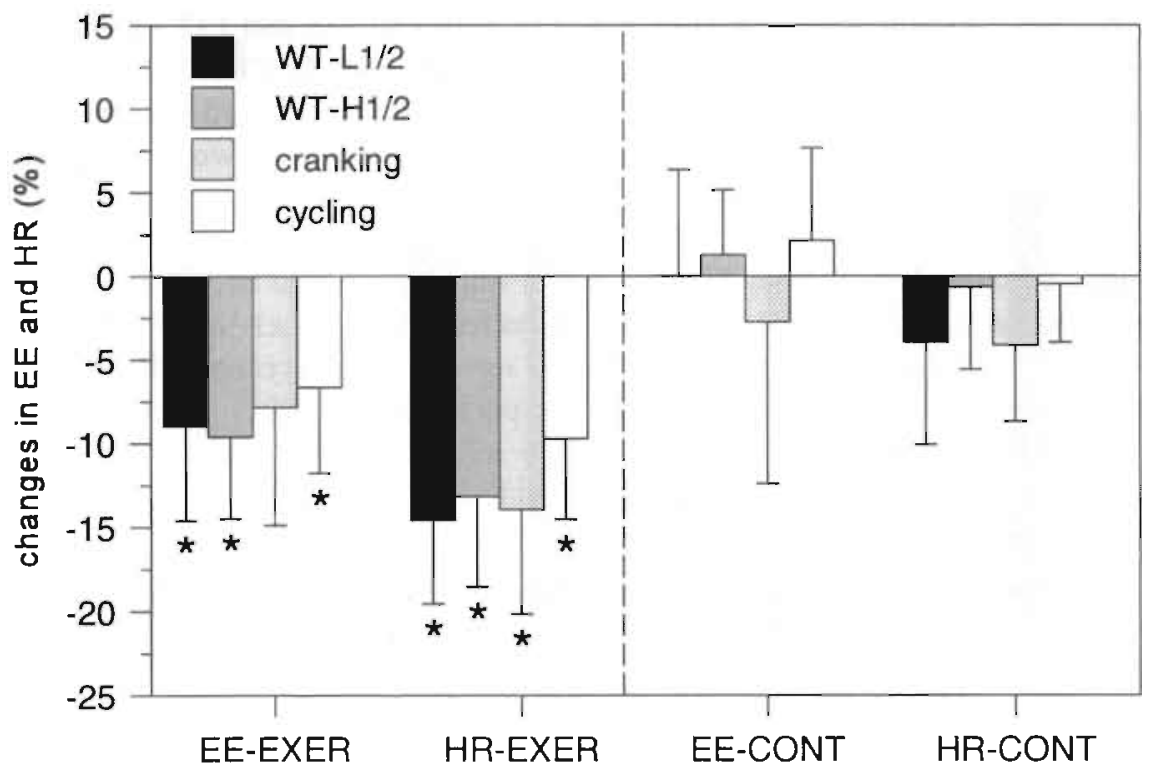

Fig. 6.4. Comparison of the relative changes (\%) in energy expenditure (EE) and heart rate (HR) between the exercise (EXER, $n=18$, left part of the figure) and the control group (CONT, $n=6$, right part of the figure). The four columns represent the average changes during the lower and the higher intensity weight-training phase (WT-L1/2 and WT.H1/2), arm cranking and cycling ( $P<0.005)$.

as relative EE and HR during the weight-training phases, arm cranking nor cycling. A two tail $t$-test for independent samples between EXER and CONT revealed a larger relative change $(\%)$ in $\mathrm{EE}$ and $\mathrm{HR}(\mathrm{P}<0.001)$ during weight training and cycling in EXER (see Fig. 6.4). During arm cranking only the decrease in HR was larger in EXER ( $P<0.001)$, whereas the change in EE did not differ between EXER and CONT $(-7.8 \pm 7.0$ versus $-2.8 \pm 9.6 \% ; \mathrm{P}=0.18)$.

Ventilation. Besides EE and $\mathrm{HR}$, also ventilation (Ve), breathing frequency $(\mathrm{Bf})$ and tidal volume (TV) changed significantly in EXER. The changes in Ve and TV as well as the changes in HR were significantly correlated with the change in EE during exercise (range $0.45-0.81$ ).

Cycling and muscular strength tests. In EXER, $\mathrm{VO}_{2} \max$ and $\mathrm{W}_{\max }$ increased significantly (17.5 and 17.1\%, P<0.001) whereas HRmax did not change (see Table 6.3). Muscular strength during the Leg extension and Bench press movement also increased significantly $(8.4$ and $10.5 \%, \mathrm{P}<0.001)$. None of these changes were correlated with the changes in fat-free mass or exercise economy. There were no changes in performance in the control group. 
Table 6.4. Initial values and changes $(\Delta)$ in the results of the cycling-and muscular strength-test in the exercise (EXER), and the control group (CONT).

\begin{tabular}{|c|c|c|c|c|c|}
\hline & \multicolumn{3}{|c|}{ EXER } & \multicolumn{2}{|c|}{ CONT } \\
\hline & initial & & $\Delta$ & initial & $\Delta$ \\
\hline $\mathrm{VO}_{2} \max \left(1 \cdot \mathrm{min}^{-1}\right)$ & $3.08 \pm 0.57$ & 0.54 & $\pm 0.30^{* *}$ & $2.92 \pm 0.28$ & $0.14 \pm 0.31$ \\
\hline$W_{\max }$ (watt) & $252 \pm 51$ & 43 & $\pm 16^{\star *}$ & $224 \pm 14$ & $14 \pm 19$ \\
\hline $\mathrm{HR}_{\max }$ (beats.min ${ }^{-1}$ ) & $191 \pm 12$ & -1.8 & \pm 6.7 & $188 \pm 10$ & $0.4+2.7$ \\
\hline Leg extension (Nm) & $170 \pm 30$ & 14.3 & $\pm 12.1^{\star \star}$ & $166 \pm 32$ & $-7.8 \pm 20.3$ \\
\hline Chest press (Nm) & $281 \pm 63$ & 29.6 & $\pm 26.1^{* *}$ & $315 \pm 103$ & $-20.8 \pm 26.3$ \\
\hline
\end{tabular}

${ }^{*} P<0.001$

Pre-session resting EE and HR. After $18 \mathrm{wk}$ of training pre-session resting EE did not change $\left(5.0 \pm 0.7\right.$ versus $\left.4.9 \pm 0.5 \mathrm{~kJ} \cdot \mathrm{min}^{-1}\right)$ whereas initial resting HR $(68.7 \pm 8.0$ beats $\left.\cdot \mathrm{min}^{-1}\right)$ decreased by $4.3 \pm 6.8$ beats $\cdot \mathrm{min}^{-1}(\mathrm{P}<0.05)$. Average EE over the entire 15 min pre-session resting period did not change $E E\left(6.2 \pm 0.7\right.$ versus $\left.6.0 \pm 0.6 \mathrm{~kJ} \cdot \mathrm{min}^{-1}\right)$ whereas HR showed a significant decrease $\left(74.5 \pm 8.0\right.$ versus $\left.70.3 \pm 9.6 \mathrm{~kJ} \cdot \mathrm{min}^{-1}\right)$. There were no changes in pre-session EE nor HR in CONT.

Recovery energy expenditure. EE returned to pre-session values within the $15 \mathrm{~min}$ recovery period (see Fig. 6.5). Total excess energy expenditure (15 min post- minus 15 min pre-session resting $\mathrm{EE}$ ) decreased after $18 \mathrm{wk}$ of weight training for session LI/2 $(27.8 \pm 9.0 \mathrm{~kJ}$ vs $19.8 \pm 10.6 \mathrm{~kJ}, \mathrm{P}<0.005)$ and session $\mathrm{Hl} / 2(48.3 \pm 16.9 \mathrm{vs} 33.1 \pm 10.6$ $\mathrm{kJ}, \mathrm{P}<0.001)$.

Workout intensity. Average training compliance, including the six training sessions in week $1-3$, was $95 \pm 7 \%$. The average number, duration and exercise heart rates of the workouts at the fitness centre did not differ between week 8-9 and week 17-18 (see Table 6.5).

Table 6.5. Number, duration and heart rate (HR) of the workouts at the fitness centre.

\begin{tabular}{lcc}
\hline & week 7-8 & week 17-18 \\
\cline { 2 - 3 } number of workouts (workout.week ${ }^{-1}$ ) & $1.9 \pm 1.5$ & $1.9 \pm 1.5$ \\
duration workout (min) & $71 \pm 11$ & $73 \pm 13$ \\
cycling HR (beats. $\mathrm{min}^{-1}$ ) & $150 \pm 10$ & $146 \pm 8$ \\
weight-training HR (beats. $\mathrm{min}^{-1}$ ) & $121 \pm 11$ & $118 \pm 8$ \\
\hline
\end{tabular}



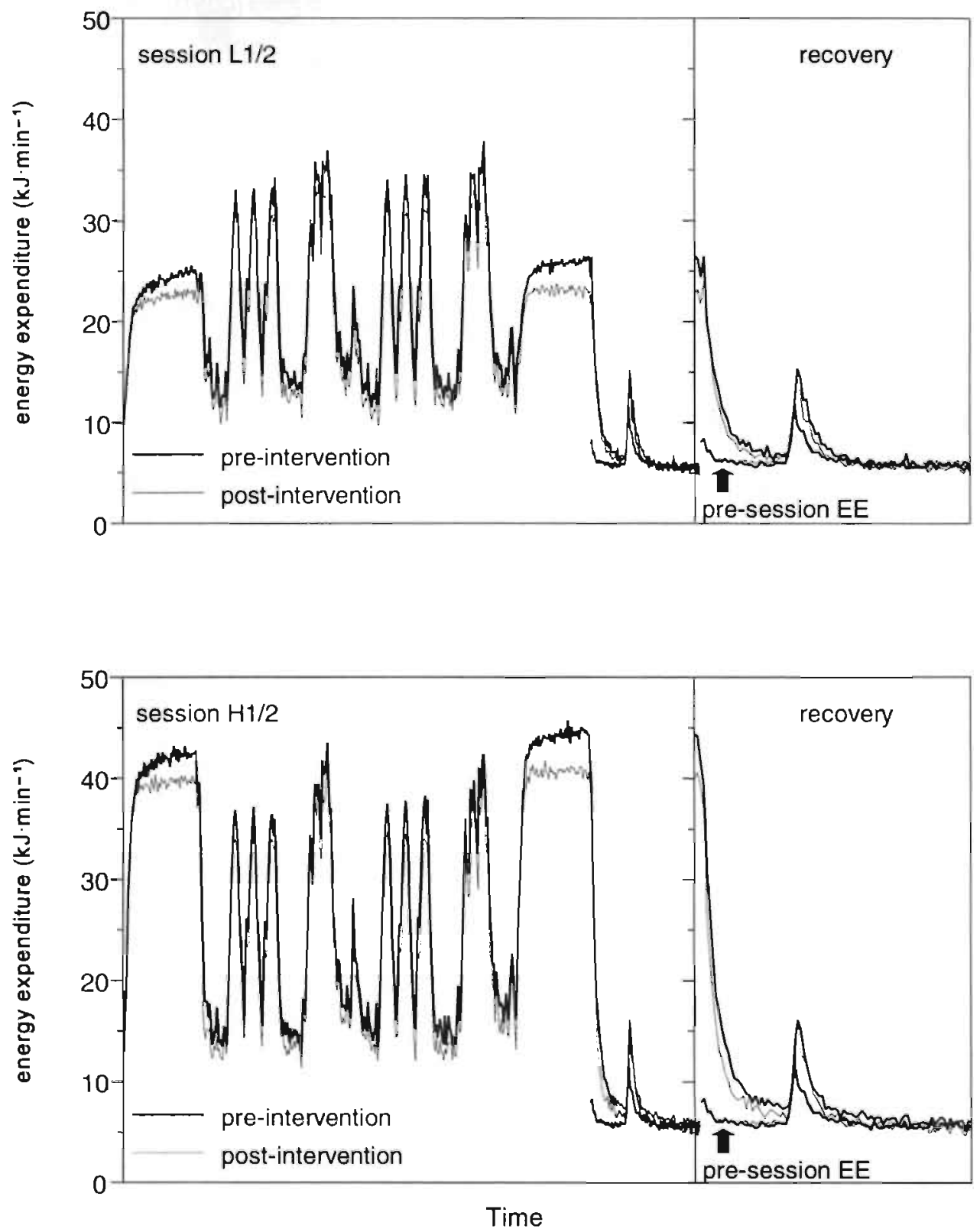

Fig. 6.5. The average pre- and post-intervention energy expenditure (EE) of two sessions that consisted of arm cranking and the lower weight-training intensity (session L1/2) and two sessions that consisted of cycling and the higher weight-training intensity (session H1/2). The right part of the figures depicts an enlargement of the $15 \mathrm{~min}$ pre-session and recovery data. 


\section{DISCUSSION}

Changes in physical characteristics. Although the change in fat mass in the exercise group is in concordance with weight-training-induced changes in fat mass found in the literature $\left(-0.1 \mathrm{~kg} \cdot\right.$ week $\left.^{-1}\right)(36)$, the comparable change in fat mass in the control group is an indication that seasonal changes in food habits or spontaneous activity could have played a role. The increase in fat-free mass was most likely due to the intervention since no significant change was observed in the controls.

Reproducibility of EE and HR. Pre-intervention comparison in EXER of the test-retest sessions revealed a decreased $\mathrm{EE}$ and HR during the wcight-training and warming-up phase of session L2. Post-intervention comparison of the test-retest sessions revealed a decreased EE during the weight-training phase of session L2. In other words, pre- as well as post-intervention session L1 (first session to collect data) showed a slightly increased cardio-respiratory response. This small but systematic increase in cardiorespiratory data during the first session was most likely due to psychogenic factors such as subject unfamilarity with the spirometric equipment (10).

Pre- versus post-intervention EE and HR. A post-intervention decrease in EE and HR was found irrespective the weight-training intensity or mode of exercise (arm cranking or cycling). The absence of a change in cardiorespiratory data in the control group suggests that the changes in EE and HR could be due to the training program. However, the size of the control group was substantially smaller than the exercise group (consisted only of six subjects). This smaller sample size affects the level of significance. Therefore, additionally the changes in EE and HR were compared between EXER and CONT. To correct for inter-individual differences in initial EE and $\mathrm{HR}$, the changes were expressed relative to pre-intervention values (see Fig. 6.4). Analysis revealed a consistent larger decrease in EE and HR in the exercise group (except EE arm cranking), corroborating a training induced effect on exercise economy and heart rate. The lack of a significant larger change in EE during arm cranking was largely due to one control subject who experienced a lot of problems to complete the 10 minutes of arm cranking during the pre-intervention sessions. Although the lowest load ( $42 \mathrm{~W}$ ) was used, he demonstrated one of the highest EEvalues $\left(26.6 \mathrm{~kJ} \cdot \mathrm{min}^{-1}\right)$. During the post-intervention sessions, this subject demonstrated only a decrease in EE during arm cranking (16\%: one of the largest decreases) whereas EE during both weight-training intensities and cycling increased by approximately $4 \%$. After excluding this subject from the analysis, the EXER revealed also a larger decrease in EE during arm cranking $(-7.8 \%$ versus $0.2 \%, \mathrm{P}<0.05)$.

The training program (intervention). The overall decrease in EE and HR during weighttraining exercises as well as continuous arm cranking and cycling suggests that the metabolic and circulatory adaptations due to the intermittent weight-training program consist of exercise specific as well as non-specific (i.e. central) adaptations. This is in concordance with previous studies $(6,21)$ revealing a significant decrease in 
$\mathrm{VO}_{2}$ submax and HRsubmax during exercise performed with both trained as well as untrained muscles but with a significantly greater change in the trained muscles. The present training program, however, was not as specific as suggested. Besides involving a wide variety of different muscles, the workouts did not exclusively consist of typical weight-training exercises but furthermore included about $20 \mathrm{~min}$ of cycling per session. Consequently, a combined intermittent/continuous exercise program would be a more appropriate description of the intervention. So, part of the overall (nonspecific) improvement in exercise economy should be attributed to the combined nature of the program. This combined nature could also have caused the increase in $\mathrm{VO}_{2} \max$ and $\mathrm{W}_{\max }$ which is rarely found in weight-training interventions (30). The improvement in arm cranking economy, on the other hand, could only be due to nonspecific adaptation since arm cranking was the only exercise that was not part of the intervention program. The deficiency of an exercise specific adaptation would explain the somewhat less pronounced improvement in arm cranking economy.

Mechanisms affecting exercise economy. Exercise economy needs a multi-dimensional approach since physiological. biomechanical, biochemical, psychomotor and some less classifiable factors are known to affect or change exercise economy (4). An approach to categorize these factors is to distinguish factors that affect the energy needed to overcome external resistance (i.e. external energy, mainly of biomechanical origin), factors affecting the energy used in the production of external energy (i.e. internal energy; mainly physiological and chemical), and some remaining factors (e.g. psychological) (2). So, changes in energy demand for external energy, internal energy or both, will affect exercise economy.

External energy. Although training weights were identical during the pre- and postintervention economy sessions, external resistance could be changed by an altered body mass since $50 \%$ of the weight-training exercises were weight bearing. This is, however, not applicable because body mass did not change. A change in segmental mass distribution due to a change in body composition is a second factor that could alter the amount of work. No data on the distribution of the mass among limb segments were collected. However, weight reduction from the extremities is less likely because in men the loss of adipose tissue appears to be primarily central (abdominal) (13). It will be even more likely that segmental mass increased because in men weight training induces a muscle mass increase in the extremities $(3,11,19)$. The small number and low pace of the movements compared to running makes it also less apparent that this change in segmental mass distribution would substantially affect exercise economy. Changes in other biomechanical variables like technique were minimized by the use of stringent standardization. Exercise kinematics during the weight-training phase were controlled by strict technique demands and computerized pacing whereas arm cranking and cycling were circular movements performed at a standard RPM (16) and could therefore not change like for example stride length during running. 
Internal energy. Heart rate and ventilation (Ve) are both essential in the oxygen delivery to the working muscles. The training program decreased exercise $\mathrm{HR}$ and Ve and also pre-session resting HR. As in other studies (24), changes in exercise HR and $V$ e were significantly related to changes in the energy costs of exercise because muscle blood flow and ventilation are tightly coupled to the metabolic demand of the exercise. The decrease in $\mathrm{Ve}$ in the present study was due to an decrease in both breathing frequency as well as tidal volume, except during arm cranking where only tidal volume decreased. Since the work of HR and Ve constitute only $\pm 8-9 \%$ of the total energy cost of exercise (2), the decrease in these two variables can only explain a small part of the improvement in exercise economy.

A more peripheral acting mechanism that could have improved exercise economy is neural adaptation (motor learning, coordination) (28). Although movements were selected that required minimal locomotory skills, it can not be excluded that the contribution of synergistic/antagonistic muscles and prime movers was changed due to the intervention (i.e. inter-muscular coordination). A change in synergistic activity especially goes for arm cranking where rotations of the trunk were more difficult to control. On the other hand, EE during cycling also decreased whereas the majority of subjects used bicycles on a daily basis. Also changes in intra-muscle coordination could have affected exercise economy. A study of Ploutz et al (25) revealed that a nine week resistance-training program reduced the amount of muscle used to lift standard loads. They suggested neural factors to be responsible.

A locomotory induced change in the ability to store and reutilize elastic energy derived from eccentric stretch of the elastic components of the muscle (5) was not likely to occur since the movements were paced by a computer (17) and the speed of the movements was low $\left( \pm 2 \mathrm{sec} \cdot\right.$ movement $\left.^{-1}\right)$.

Remaining factors. From endurance as well as weight-training studies it is known that exercise economy exhibits an inverse relationship to intensity of exercise. Several possibilities may explain this finding: an increased contribution from less efficient fast twitch muscle fibers; an increase in metabolism not directly related to the external work (e.g. greater activity of stabilizer muscles and breathing muscles or tremor in the limbs) or an increased release of catecholamines $(9,16-18,20,31)$. Since $V O$, max and muscular strength both increased, the intensity of the exercises subsequently decreased. However, analysis revealed no relationship between the increase in physical performance and the decrease in exercise EE although this lack of relationship also could be due to the type of strength test (non-specific isokinetic lest) and the relative low intensity and small decrease in intensity during cycling (57 \pm 7 versus $48 \pm 6 \% \mathrm{~W}_{\max }, \mathrm{P}<0.001$ ). Based on the workout heart rates, the intensity of the workouts at the fitness club remained the same and were of a higher intensity than the post-intervention sessions in the laboratory. Another factor that could have improved exercise economy is a transformation among the fast-twitch fiber subtypes as demonstrated in other weight-training intervention studies $(1,25)$.

A separate component of exercise EE is resting EE. Because exercise economy is expressed as gross EE, a change in resting EE could affect exercise economy. Pre- and 
post-intervention comparison revealed no change in resting EE. This is in concordance with previous findings (32). Although the effect of training on resting EE is somewhat controversial (26), no study revealed a training induced decrease in resting EE in weight stable subjects. Therefore, it is not likely that a change in resting EE would decrease exercise EE. However, we must keep in mind that base-line subtraction (i.e., minus resting EE) does not result in the energy used for the performance of external work since exercise baseline values change with increasing work rate (29) and training status (see $\Delta \mathrm{HR}$ and $\Delta \mathrm{Ve}$ ). An experimental design in which resting EE could have played a significant role was used by Morgan et al (22). In a cross-sectional comparison between trained and untrained subjects, the aerobic demand of running was expressed as $\mathrm{ml} \mathrm{O}_{2} \cdot \mathrm{kg}^{-1} \cdot \mathrm{km}^{-1}$. Because untrained subjects run at slower speeds the calculated $\mathrm{ml} \mathrm{O}_{2} \cdot \mathrm{kg}^{-1} \cdot \mathrm{km}^{-1}$ also included the resting energy expenditure over a longer period what negatively affected exercise economy. So, in the latter design it would be more appropriate to compare the energy expended above resting EE. If exercise economy in the present study was expressed as gross EE minus resting EE, the relative improvement during the low and high weight-training exercise, arm cranking and cycling would further increase to $11.5,12.0,9.7$ and $7.7 \%$.

Kinetics of EE. From the pattern of EE (see Fig. 6.5) it appears that the improvement in weight-training economy was not only due to a decreased $E E$ during exercise (i.e. smaller peaks) but also a better recovery during the rest intervals. The latter was confirmed by a significant decrease in excess postexercise EE. The pattern of EE during arm cranking and cycling suggest that there is a decrease in EE in the slow component ( $>3$ min exercise) as known from other studies (15) as well as an overall decrease in EE. Since the exercising muscle is the predominant site of the slow component (27), the decrease in the slow component suggest additionally a peripheral adaptation in the working muscle.

In conclusion, 18 weeks of weight training decreased energy expenditure during intermittent weight-training exercises, and continuous arm cranking and cycling. The improvement in exercise economy was most likely due to a combination of central (decrease heart rate, ventilation and an improved coordination) and peripheral (intramuscular) adaptations and decreased testing intensity as a consequence of the increase in muscular strength and aerobic capacity.

\section{REFERENCES}

1 Adams, G. R., B. M. Hather, K. M. Baldwin, G. A. Dudley. Skeletal muscle myosin heavy chain composition and resistince training. J. Appl. Physiol. 74(2):911-915, 1993.

2 Bailey, S. P., R. R. Pate. Feasibility of improving running economy. Sports Med. 12(4):228-236, 1991. 
3 Ballor, D. L., V. L. Katch, M. D. Becque, C. R. Marks. Resistance weight training during caloric restriction enhances lean body weight maintenance. Am. J. Clin. Nutr. 47:19-25, 1988.

4 Cavanagh, P. R., R. Kram. The efficiency of human movement-a statement of the problem. Med. Sci. Sports Exerc. 17(3):304-308, 1985.

5 Cavanagh, P. R., R. Kram. Mechanical and muscular factors affecting the efficiency of human movement. Med. Sci. Sports Exerc. 17(3):326-331, 1985.

6 Clausen, J. P., K. Klausen, B. Rasmussen, J. Trap-Jensen. Central and peripheral circulatory changes after training of the arms or legs. Am. J. Physiol. 225(3):675$682,1973$.

7 Conley, D. L., G. S. Krahenbuhl, L. N. Burkett. Training for aerobic capacity and running economy. Phys. Sportsmed. 9(4):107-115, 1981.

8 Conley, D. L., G. S. Krahenbuhl, L. N. Burkett, A. L. Millar. Following Steve Scott: Physiological changes accompanying training. Phys. Sportsmed. 12(1):103$106,1984$.

9 Coyle. E. F., L. S. Sidossis, J. F. Horowitz, J. D. Beltz. Cycling efficiency is related to the percentage of Type I mucle fibers. Med. Sci. Sports Exerc. 24(7):782-788, 1992

10 Crews, D. J. Psychological state and running economy. Med. Sci. Sports Exerc. 24(4):475-482, 1992.

11 Cureton, K. J., M. A. Collins. D. W. Hill, F. M. Mcelhannon. Muscle hypertrophy in men and women. Med. Si'i. Sports Exerc. 20(4):338-344, 1988.

12 Daniels, J. T. A physiologist's view of running economy. Med. Sci. Sports Exerc. 17(3):332-338, 1985.

13 Despres, J.-P., A. Tremblay, A. Nadeau, C. Bouchard. Physical training and changes in regional adipose tissue distribution. Acta Med.Scand. Suppl.:723:-205, 1988.

14 Fuller, N. J., S. A. Jebb, M. A. Laskey, W. A. Coward, M. Elia. Four-component model for the assessment of body composition in humans: comparison with alternative methods, and evaluation of the density and hydration of fat -free mass. Clin. Sci. 82:687-693, 1992

15 Gaesser, G. A. Influence of endurance training and catecholamines on exercise VO2 response. Medicine and Science in Sports and Exercise 26(11):1341-1346, 1994.

16 Gaesser, G. A., G. A. Brooks. Muscular efficiency during steady-rate exercise: effects of speed and work rate. J. Appl. Physiol. 38(6):1132-1139, 1975.

17 Hunter, G., L. Blackman, L. Dunnam, G. Flemming. Bench press metabolic rate as a function of exercise intensity. J. Appl. Sport Sci. Res. 2(1):1-6, 1988.

18 Kalb, J. S., G. R. Hunter. Weight training economy as a function of intensity of the squat and overhead press exercise. J. Sports Med. Phys. Fitness 31:154-160, 1991.

19 Katch, F. I., S. S. Drumm. Effects of different modes of strength training on body composition and anthropometry. Clin. Sport Med. 5(3):413-459, 1986. 
20 Luthanen, P., P. Rahkila, H. Rusko, J. T. Viitasalo. Mechanical work and efficiency in treadmill running at earobic and anaerobic thresholds. Acta Physiol Scand 139:153-159, 1989.

21 McKenzie, D. C., E. L. Fox, K. Cohen. Specificity of metabolic and circulatory responses to arm or leg interval training. Eur. J. Appl. Physiol. 39:241-248, 1978.

22 Morgan, D. W., D. R. Bransford, D. L. Costill, J. T. Daniels, E. T. Howley, G. S. Krahenbuhl. Variation in the aerobic demand of running among trained and untrained subjects. Med. Sci. Sport Exerc. 27(3):404-409, 1995.

23 Morgan, D. W., P. E. Martin, G. S. Krahenbuhl. Factors affecting running economy. Sports Med. 7:310-330, 1989.

24 Pate, R. P., C. A. Macera, S. P. Bailey, W. P. Bartoli, K. E. Powell. Physiological, anthropometric, and training correlates of running economy. Med. Sci. Sports Exerc. 24(10):1128-1133, 1992.

25 Ploutz, L. L., P. R. Tesch, R. L. Biro, G. A. Dudley. Effect of resistance training on muscle use during exercise. J. Appl. Physiol. 76(4):1675-1681, 1994.

26 Poehlman, E. T., C. L. Melby, M. I. Goran. The impact of exercise and diet restriction on daily energy expenditure. Sports Med. 11:78-101, 1991.

27 Poole, D. C. Role of exercising muscle in slow component of VO2. Med. Sci. Sports Exerc. 26(11):1335-1340, 1994.

28 Sale, D. G. Neural adaptation to resistance training. Med. Sci. Sports Exerc. 20(5):S135-S145, 1988.

29 Stainsby, W. N., B. L. Gladden, J. K. Barclay, B. A. Wilson. Exercise efficiency: validity of baseline subtractions. J. Appl. Physiol. 48(3):518-522, 1980.

30 Stone, M. H., S. J. Fleck, N. Travis Triplet, W. J. Kraemer. Health and performance-related potential of resistance training. Sports Med. 11(4):210-231, 1991.

31 Thompson, J. K., P. Blanton. Energy conservation and exercise dependence: a sympathetic arousal hypothesis. Med. Sci. Sports Exerc. 19(2):91-99, 1987.

32 Van Etten, L. M. L. A., K. R. Westerterp, F. T. J. Verstappen. Effect of weighttraining on energy expenditure and substrate utilization during sleep. Med. Sci. Sports Exerc. 27(2):188-193, 1995.

33 Weir de, J. B. New methods for calculating metabolic rate with special reference to protein metabolism. J. Physiol. 109:1-9, 1949.

34 Westerterp, K. R., L. Wouters, W. D. Marken Lichtenbelt. The Maastricht protocol for the measurement of body composition and energy expenditure with labeled water. Obesity Research 3(suppl 1):49-57, 1995.

35 Williams, T. J., G. S. Krahenbuhl, D. W. Morgan. Daily variation in running economy of moderately trained male runners. Med. Sci. Sports Exerc. 23(8):944948, 1991.

36 Wilmore, J. H. Variations in physical activity habits and body composition. Int. J. Obesity 19(sup 4):S107-S112, 1995. 


\section{Chapter 7}

\section{The energy expenditure-heart rate relationship during weight training}

Ludo M.L.A. van Etten, Frans T.J. Verstappen and Klaas R. Westerterp Department of Human Biology and Department of Movement Sciences, Maastricht University, Maastricht, The Netherlands submitted

\section{ABSTRACT}

The aim of the present study was to examine whether energy expenditure during weight training could be accurately predicted on the energy expenditure (EE) heart rate $(H R)$ relationship derived from the wide range of $E E$ and $H R$ values observed during weight training. A group of 26 male subjects performed 4 computer paced weight-training sessions using siandard weights. The initial training weights (lower intensity) were increased with $50 \%$ during session 3 and 4 (higher intensity). Average $E E$ and $H R$ of the lower and higher intensity were $19.6 \pm 2.1,22.2 \pm 2.2 \mathrm{~kJ} \cdot \mathrm{min}^{-1}$ and $98.8 \pm 14.0,110.5 \pm 16.6$ beats. $\mathrm{min}^{-1}$. Within one intensity, EE was predicted accurately (average error 4\%) on the EE/HR-relationship of the duplicate session. The EE/HR-relationship derived from the lower intensity overestimated EE systematically at the higher intensity and vice versa (average error 11\%). This systematic inaccuracy was highly correlated with the difference in average HR between the two intensities. Furthermore, there appeared to be a drift in relative HR during a weighttraining session revealing an exercise duration effect on the EE/HR-relationship. These results demonstrated that an intermittent, non-incremental protocol yields an EE/HR-relationship that predicts EE during combined arm and leg work with a reasonable level of accuracy. The accuracy will further improve as similar exercise and calibration intensities and durations will be used. 


\section{INTRODUCTION}

The linear relationship between energy expenditure (EE) and heart rate (HR) is frequently used to predict EE during physical exercise. Heart rate monitoring is in fact the only method to determine. EE during short term activities in which spirometry (i.e., indirect calorimetry) is not feasible or would limit the freedom of movement. Weight training is such a type of exercise that demands a fair amount of unrestricted body movement since different workstations must be visited and standing, sitting, supine as well as prone body positions are used to exercise different muscle groups.

The EE/HR-relationship is usually determined during an incremental (staged) exercise test measuring heart rate and gas exchange simultaneously. However, one mode of exercise is not always sufficient to decide an accurate EE/HR-relationship. Previous studies that compared leg versus arm exercise and weight bearing (running, stepping) versus weight-supported (cycling, rowing) modes of exercise, revealed that the EE/HR-relationship is task specific $(2,9,12,15,16)$. Since weight training consists of a wide variety of exercises including upper and lower body activity, an adapted protocol should be used to determine an adequate EE/HR-relationship.

Another characteristic of weight training is that it is not an incremental or continuous type of activity, like running or cycling, but an alternation of relative short exercise periods with recovery-intervals. Therefore, a weight-training specific EE/HRrelationship should not only include the primary fast component of cardiorespiratory adaptation to exercise but should also take the fast component $(<60 \mathrm{~s})$ of cardiorespiratory recovery into account. Especially this latter condition makes the use of an incremental calibration protocol to determine an accurate EE/HR-relationship for weight-training activities questionable.

This study was conducted to investigate whether the wide range of heart rate and energy expenditure observed within a single weight-training session -including arm and leg exercises as well as adaptation and recovery- yield an accurate EE/HR-relationship to predict EE during weight training at similar and dissimilar intensities. A group of 26 healthy men performed 4 weight-training sessions using two intensities. The within-subject linear regression equations of EE over HR for each session/intensity (calibration lines) were used to predict EE during the remaining weight-training sessions/intensity and compared with the measured EE.

\section{METHODS}

Subjects. After obtaining informed consent, 32 healthy male subjects participated in this study and performed six training sessions. Six subjects were excluded from analysis because they were not able to complete the training sessions using the prescribed training load (they failed at the higher weight-training intensity). From the remaining group of 26 subjects previous weight training experience ranged from: no practice $(n=4), 4$ months $(n=14)$ and more than 2 years $(n=8)$ of weight training experience. Their physical characteristics are presented in Table 7.1. 
Table 7.1. Physical characteristics (means $\pm S D$ ) of the subjects $(n=26)$

\begin{tabular}{lcc}
\hline \multicolumn{1}{c}{ variables } & mean \pm SD & range (min-max) \\
\cline { 2 - 2 } age $(\mathrm{yr})$ & $31.9 \pm 5.8$ & $23-41$ \\
weight $(\mathrm{kg})$ & $78.8 \pm 9.5$ & $54.3-103.5$ \\
height $(\mathrm{m})$ & $1.82 \pm 0.05$ & $175-1.97$ \\
Body Mass Index $\left(\mathrm{kg} \cdot \mathrm{m}^{-2}\right)$ & $23.6 \pm 2.5$ & $16.9-29.2$ \\
fat $(\%)$ & $17.4 \pm 5.7$ & $7.9-26.3$ \\
fat-free mass $(\mathrm{kg})$ & $64.8 \pm 7.2$ & $48.7-77.5$ \\
fat mass $(\mathrm{kg})$ & $13.9 \pm 5.5$ & $5.1-26.3$ \\
\hline
\end{tabular}

Training sessions. Each weight-training (WT) session included 3 sets of 15 repetitions of the following exercises: bench press, lunges, dumbbell pull, squat, and the overhead lats pulley ( 3 upper body and 2 lower body exercises). This sequence of five exercises was completed twice to increase the volume of the session without increasing the number of different exercises (see Table 7.2). Total duration of the WT phase was 42 minutes. Since a single repetition of the lunges consisted of an alternating left and right step forward, total duration of this exercise was approximately twice the time used by other exercises. Hence, exercise time was equally split up between the upper and lower part of the body. The work to rest ratio was 1:1. A computer-generated acoustic signal dictated the pace during exercise and the duration of the resting intervals. Variability in technical performance was minimized by using strict technique demands. The initial training load during the WT phase of session 1 to 4 (lower WT-intensity) was increased by raising training weights with approximately $50 \%$ during session 5 and 6 (higher WT-intensity). As is customary, subjects also performed a warming up and a cooling down (both $10 \mathrm{~min}$ ). During the first four sessions arm ergometry (i.e., arm cranking; 48 watt/ $60 \mathrm{rpm}$, Ergostat Universal, Switzerland) was used, whereas session five and six used leg ergometry (i.e., cycling; 150 watt/ 75rpm, Jaeger ER800) as warming-up and cooling-down exercise (for reasons not relevant to the current research question). The difference in type of warming up did not significantly affect the EE/HR-relationship during the 42 minutes of weighttraining exercise (see discussion).

Session measurements. From the total of 6 training sessions, first two sessions werc used to accommodate the subjects to the laboratory setting and computerized pacing. No measurements were carried out and the subjects had full freedom of movement since they were not connected to the gas analyzer. The remaining four measurement sessions consisted of two sessions performed at the lower WT-intensity (L1, L2) and two sessions performed at the higher WT-intensity $(\mathrm{H} 1, \mathrm{H} 2)$. The balanced increase in training loads was especially intended for the untrained subjects. All sessions were 
conducted at the same time in the morning to minimize the residual effects of physical activity, food intake and circadian variation, on energy expenditure and were scheduled within a period of two weeks. Furthermore, subjects were asked to refrain from any exercise or strenuous physical activity 36 hours before each session and to eat similar breakfasts. Data collection on gas-exchange and heart rate started simultaneously with the warming-up exercise and stopped after the cooling down.

Gas-exchange was continuously measured by open circuit spirometry using an automated system (Oxycon Beta, Mijnhardt). Respiratory parameters were calculated over an average of 8 breath cycles and reported every 10 seconds. Gas analyzers were automatically calibrated before each experiment with standard gas mixtures. Gas-exchange was converted to energy expenditure using the Weir formula (17).

Heart rate (HR) was continuously monitored using a Polar Sport Tester PE3000 and averaged over 10 seconds intervals.

Table 7.2. Protocol and sequence of the six training sessions.

\begin{tabular}{|c|c|c|c|c|c|c|c|}
\hline & Exercise & $\begin{array}{c}\text { session } \\
1 \\
\end{array}$ & $\begin{array}{c}\text { session } \\
2 \\
\end{array}$ & $\begin{array}{c}\text { session } \\
3(\text { L1) }\end{array}$ & $\begin{array}{c}\text { session } \\
4(\text { L2 })\end{array}$ & $\begin{array}{c}\text { session } \\
5(\mathrm{H} 1)\end{array}$ & $\begin{array}{c}\text { session } \\
6(\mathrm{H} 2)\end{array}$ \\
\hline \multicolumn{2}{|c|}{ warming up (10 min) } & cranking & cranking & cranking & cranking & cycling & cycling \\
\hline & bench press $(\mathrm{kg})$ & 10 & 10 & 10 & 10 & 15 & 15 \\
\hline & lunges $(\mathrm{kg})$ & 25 & 25 & 25 & 25 & 35 & 35 \\
\hline \multirow[t]{3}{*}{$2 x$} & dumbell pull (kg) & $2 \times 4$ & $2 \times 4$ & $2 \times 4$ & $2 \times 4$ & $2 \times 5^{1 / 2}$ & $2 \times 51 / 2$ \\
\hline & squat $(\mathrm{kg})$ & 30 & 30 & 30 & 30 & 45 & 45 \\
\hline & lats pulley $(\mathrm{kg})$ & $14 \frac{1}{4}$ & $141 / 4$ & $14 \%$ & $141 / 4$ & 20 & 20 \\
\hline \multicolumn{2}{|c|}{ cooling down (10 $\mathrm{min})$} & cranking & cranking & cranking & cranking & cycling & cycling \\
\hline
\end{tabular}

The duration for the warming-up and cooling-down was 10 minutes. The load and pedaling rate during cycling and arm-cranking were 150 watt/ $75 \mathrm{rpm}$ and 48 watt/ $60 \mathrm{rpm}$, respectively. The duration of the weight-training phase was 42 minutes. Training weights are expressed in $\mathrm{kg}$. Session 1 and 2 were used for accommodation. During session 3.6 (L1-H2) cardiorespiratory data were collected.

Body composition. All body characteristics were measured the morning after a controlled overnight stay at the university where subjects abstained from eating for 14 hours. Body mass was measured after voiding to an accuracy of $\pm 5 \mathrm{~g}$ on an electronic scale (Mettler, E1200). Body density was measured using the hydrostatic weighing technique with simultaneous measurement of residual lung volume with the helium dilution technique (Volugraph 200, Mijnhardt). Percent fat was calculated from body density assuming a FFM and FM density of 1.097 and $0.901 \mathrm{~kg} \cdot \mathrm{l}^{-1}$, respectively.

Statistical analysis. Paired $t$-tests were used to analyze differences between repeated measurements and between measured and predicted EE-values. Regression analysis was used to determine the relationship between HR and EE. Statistical significance was set at $\mathrm{P}<0.05$. 


\section{RESULTS}

Pattern of the EE/HR-relationship. A time plot of average EE and HR $(n=26)$ clearly shows the parallel pattern/relationship of both parameters (see Fig. 7.1). Average Pearson correlation coefficients for sessions $\mathrm{L} 1$ to $\mathrm{H} 2$ were respectively $0.81 \pm 0.05$, $0.79 \pm 0.06,0.83 \pm 0.07$ and $0.83 \pm 0.05$.

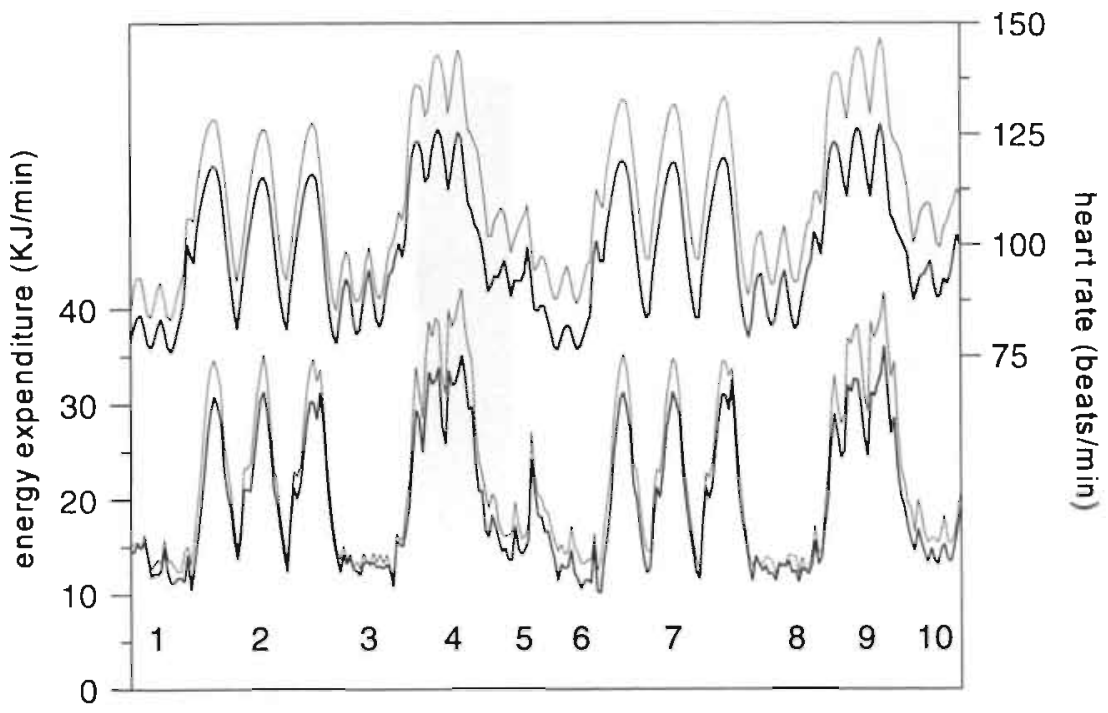

Fig. 7.1. Average energy expenditure (lower two lines, 10-seconds time intervals, $n=26$ ) and heart rates values (upper two lines) during the lower intensity (bold lines) and the higher intensity (thin lines). The numbers mark the 10 different weight-training exercises.

$E E$ and $H R$ during weight training. Energy expenditure during the WT phase of session $\mathrm{L} 1$ to $\mathrm{H} 2$ was $19.9 \pm 2.3,19.3 \pm 2.0,22.2 \pm 2.2$ and $22.1 \pm 2.2 \mathrm{~kJ} \cdot \mathrm{min}^{-1}$, respectively. The corresponding heart rate values were $100.5 \pm 14.9 .97 .1 \pm 13.8,110.3 \pm 17.5$ and $110.7 \pm 16.0$ beats $\mathrm{min}^{-1}$ (see Fig. 7.2). Although sessions L1 and L2 had an identical training load, analyses revealed a significant difference in $\mathrm{EE}(\mathrm{P}<0.001)$ as well as $H R$ $(P<0.05)$ between sessions $\mathrm{Ll}$ and $\mathrm{L} 2$. There were no differences in EE or HR between sessions $\mathrm{Hl}$ and $\mathrm{H} 2$. The average EE and $\mathrm{HR}$ of both sessions within the lower (L1/L2) and higher intensity (H1/H2) were $19.6 \pm 2.1,22.2 \pm 2.2 \mathrm{~kJ} \cdot \mathrm{min}^{-1}$ and $98.8 \pm 14.0,110.5 \pm 16.6$ beats $\cdot \mathrm{min}^{-1}$, respectively.

Predicted EE during the WT phases. To check whether the regression equation (EQ) of EE over HR during the WT phase of a single session (EQ-L1 to EQ-H2) could accurately predict EE during the WT phases of the remaining weight-training sessions, the measured values were compared with the predicted values. Within one intensity there 
were no significant differences between measured and predicted values (see Table 7.3). The average difference between measured and predicted EE was $\approx 4 \%$.

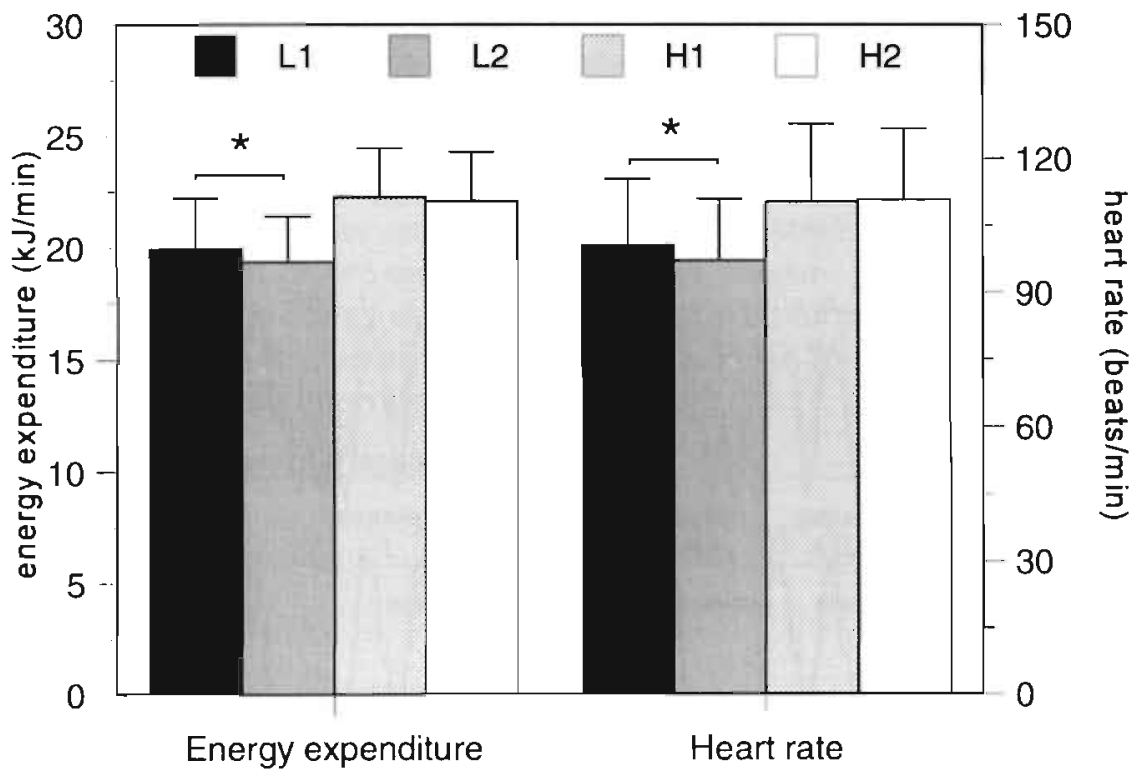

Fig. 7.2. Average energy expenditure (mean $\pm S D, k J \cdot \mathrm{min}^{-1}$ ) and heart rate (beats $\cdot \mathrm{min}^{\top}$ ) during the four weighttraining sessions. Training load (lower intensity) during the first two sessions (L1 and L2) was increased with $\approx 50 \%$ (higher intensity) during the last two sessions ( $\mathrm{H} 1$ and $\mathrm{H} 2$ ). ${ }^{*} \mathrm{P}<0.05$.

Table 7.3. Comparison between the measured and predicted energy expenditure (EE) of the single (L1, $L 2, H 1$ and $H 2$ ) and the pooled ( $L 1 / 2$ and $H 1 / 2)$ sessions.

\begin{tabular}{lcc:c:l}
\hline session & HR (beats $\left.\cdot \mathrm{mln}^{-1}\right)$ & EE $\left(\mathbf{k J} \cdot \mathbf{m i n}^{-1}\right)$ & predicted EE & equation (EQ) used \\
\hline $\mathrm{L} 1$ & 100.4 & 19.9 & 20.5 & $\mathrm{EQ}-\mathrm{L} 2$ \\
$\mathrm{~L} 2$ & 97.0 & 19.3 & 18.6 & $\mathrm{EQ}-\mathrm{L} 1$ \\
$\mathrm{H} 1$ & 110.3 & 22.2 & 21.8 & $\mathrm{EQ}-\mathrm{H} 2$ \\
$\mathrm{H} 2$ & 110.7 & 22.1 & 22.6 & $\mathrm{EQ}-\mathrm{H} 1$ \\
$\mathrm{~L} 1 / 2$ & 98.7 & 19.6 & $17.3^{*}$ & $\mathrm{EQ}-\mathrm{H} 1 / 2$ \\
$\mathrm{H} 1 / 2$ & 110.5 & 22.2 & $24.3^{*}$ & $\mathrm{EQ}-\mathrm{L} 1 / 2$ \\
\hline
\end{tabular}

'P $P 0.005$

Since there were no differences within intensities, data were pooled and regression equations were derived from data of both sessions within one intensity (EQ-L1/2, $\mathrm{EQ}-\mathrm{H} 1 / 2$ ). Average measured EE of the lower (WT-Ll/2) and higher intensity (WT$\mathrm{H} 1 / 2)$ versus the predicted values were $19.6 \pm 2.1$ vs $17.3 \pm 2.4$ and $22.2 \pm 2.2$ vs 24.3 $\pm 3.3 \mathrm{~kJ} \cdot \mathrm{min}^{-1}$, respectively $(\mathrm{P}<0.001)$. The pooled equations (EQ-L1/2 and EQ-H1/2) did not accurately predict average EE during the dissimilar intensity. Equations: derived from the lower WT-intensity (EQ-L1, EQ-L2, EQ-L1/2) systematically overestimated EE during the higher intensity and vice versa (10.3 and $-12.1 \%)$. This 
absolute $\left(\mathrm{kJ} \cdot \mathrm{min}^{-1}\right)$ and relative (\%) inaccuracy was highly correlated $(\mathrm{r}=0.87$, $\mathrm{P}<0.001$ ) with the difference in average heart rate between the two intensities (range 3.5-22.9 beats $\left.\mathrm{min}^{-1}\right)$. After correction for this relationship between accuracy and difference in heart rate the average difference between measured and predicted EE was $3.3 \%$ (see Fig. 7.3).

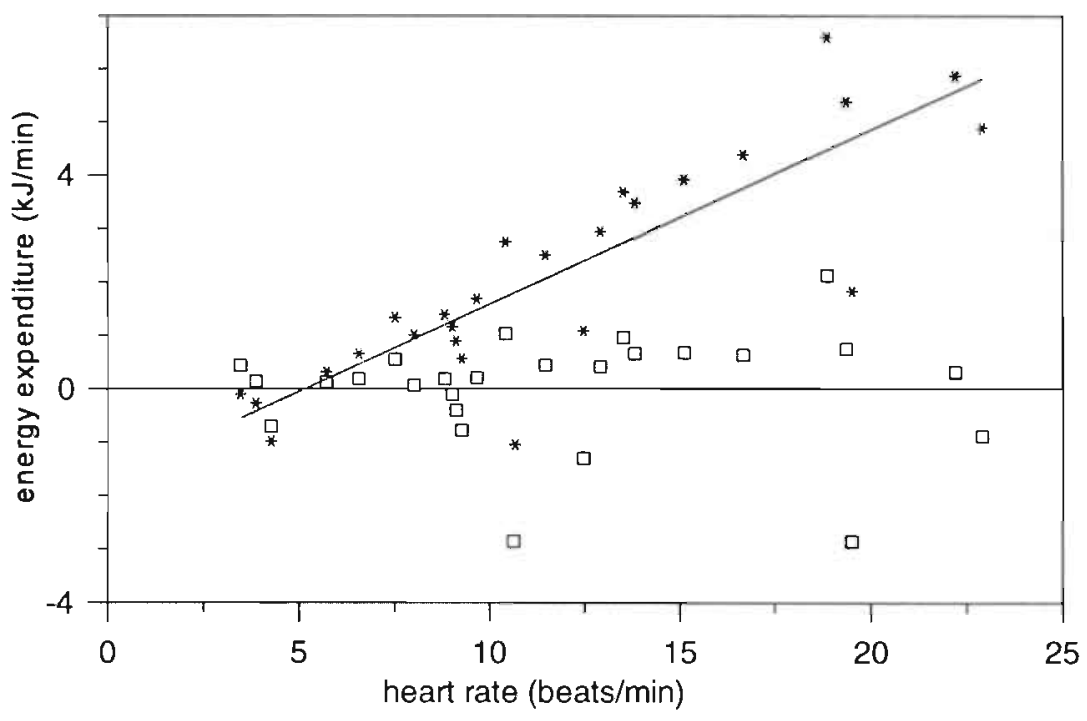

Fig. 7.3. The differences ( $*$ ) between the predicted and the measured energy expenditure of the higher weighttraining intensity plotted against the difference in heart rate between both intensities $(-1.661+0.325 \times \triangle H A$, $P<0.001, r=0.86)$. After correction for this relationship ( $\square)$, the average difference between the predicted and measured energy expenditure decreased from $10.3 \% 103.3 \%$.

Equation differences within and between intensities. The $y$-intercepts and slopes of the regression equations were compared to obtain more detailed information about the differences in the EE/HR-relationship within and between both WT-intensities. Within one WT-intensity there were no significant differences in y-intercept nor slope. Between intensities both the regression y-intercept and slope differed significantly. During the higher intensity the $y$-intercept was lower $(-20.07 \pm 5.96$ vs $-24.07 \pm 7.14$, $\mathrm{P}<0.001)$ whereas the slope was steeper $(0.406 \pm 0.063$ vs $0.422 \pm 0.067, \mathrm{P}<0.01)$ compared with the lower WT-intensity (see Fig. 7.4). The arithmetical intersection of the regression lines is situated outside the range of usual human heart rates (at 249 beats $\left.\cdot \mathrm{min}^{-1}\right)$. The range between average minimal and maximal exercise energy expenditure including all four sessions was $4.9 \pm 1.5$ to $47.4 \pm 4.9 \mathrm{~kJ} \cdot \mathrm{min}^{-1}$ and the range between average minimal and maximal exercise heart rates was $69 \pm 11$ to 153 \pm 20 beats $\mathrm{min}^{-1}$ ( see Fig. 7.4). 


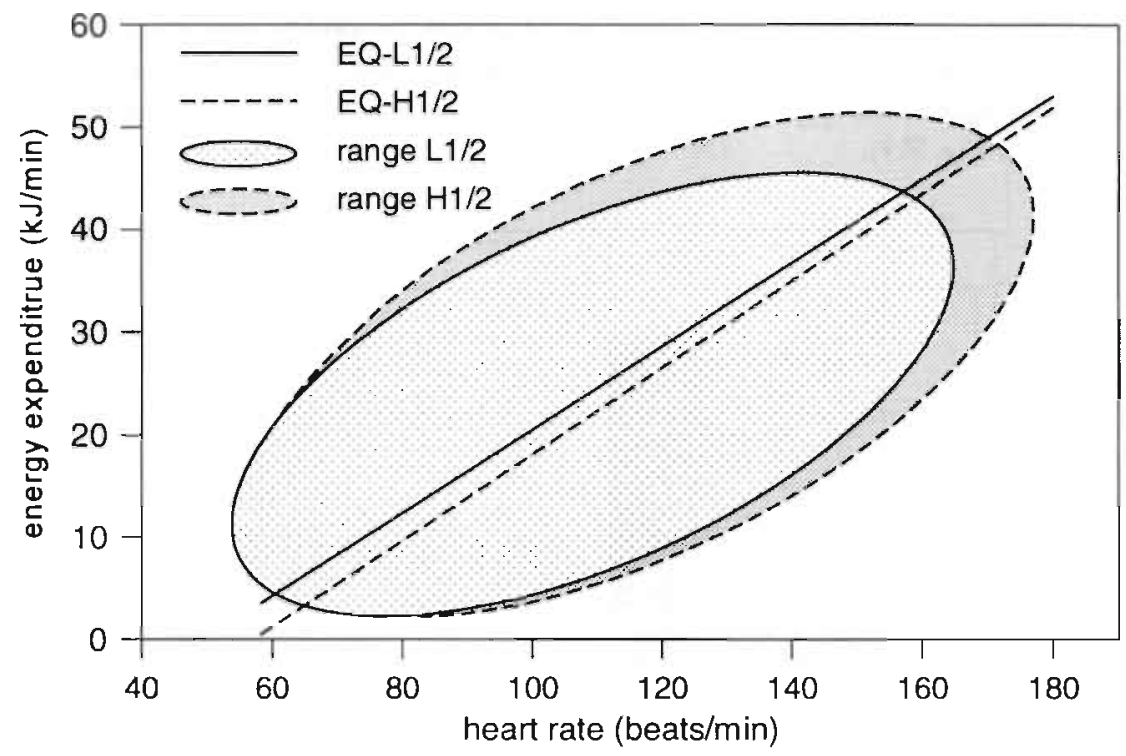

Fig. 7.4. Average regression lines of energy expenditure over heart rate during the two weight-training intensities. Both ellipses mark the average heart rate and energy expenditure ranges during the weight-training phase. The regression lines intersect outside the range of exercise heart rates (at 249 beats $-\min ^{-1}$ ).

Shift in EE/HR-relationship during a single training session. Each training session consisted of two identical blocks of five exercises. Average residual (=measured minus predicted $\mathrm{EE}$ ) differed significantly between the first five and the last five exercises. For each session the average residuals during the first part of the training were always positive $(>0)$ whereas the average residuals during the second part of the training session were negative $(<0)$. This difference in the EE/HR-relationship demonstrated as gradual drift to a higher HR per energy unit during a weight-training session. Average residual of the first versus the last five exercises of the four sessions were $0.46 \mathrm{vs}$ $-0.44,0.41$ vs $-0.40,1.06$ vs -1.01 and 0.89 vs $-0.85 \mathrm{~kJ} \cdot \mathrm{min}^{-1}$.

$E E$ and HR during the warming up and cooling down. The energy costs of arm cranking and cycling were defined as the average EE during the last five minutes of the effort. Average EE and HR during the warming up and cooling down did not differ within one exercise mode. Between arm cranking and cycling there were significant differences in average EE and HR $\left(22.6 \pm 1.7\right.$ versus $40.9 \pm 2.2 \mathrm{~kJ} \cdot \mathrm{min}^{-1}$ and $109.4 \pm 16.6$ versus $130.4 \pm 14.3$ beats $\left.\cdot \mathrm{min}^{-1}\right)$. 


\section{DISCUSSION}

The aim of the present study was to examine whether energy expenditure during weight training could be accurately predicted with the EE/HR-relationship derived from the wide range of $E E$ and $H R$ values observed during a typical weight-training session. Thus, the main interest of this study was the reproducibility of the EE/HRrelationship rather than the reproducibility of the single EE or HR values.

Predictability within one WT-intensity. Although training sessions L1 and L2 used exactly the same load, EE and HR were higher during session L1. Since changes in technical skill or confounding by technical fluctuation were not likely, this systematic difference in EE and HR during the WT phase was most likely due to the unaccustomed application of spirometry during session L1. The EE/HR-relationship, however, was not affected by significant difference since EE could be accurately predicted on the EE/HR-relationship of the other session.

During the higher intensity training there was no difference in the cardio-respiratory response between the two sessions. Therefore, the exactness of the predicted EE is no evidence that the EE/HR-relationship was similar during both sessions. Theoretically, it is possible that the two regression lines are completely different but intersect around average exercise HR's and therefore predict average EE accurately. Nevertheless, on comparison there appeared to be no difference between the regression $y$-intercept and slope within the same intensity. These results led to the conclusion that within the same intensity EE could be predicted without a systematic error with an average accuracy of approximately $4 \%$.

Predictability between the two different WT-intensities. The $50 \%$ raise in training load resulted in an average increase in EE and HR of approximately 12 and $13 \%$, respectively. This comparable change suggests that the initial EE/HR-relationship may accurately predict $\mathrm{EE}$ at a higher intensity. However, this latter suggestion is only true if the $y$-intercept would be around zero. Since the average y-intercepts were significantly smaller than zero $\left(-20\right.$ and $\left.-24 \mathrm{~kJ} \cdot \mathrm{min}^{-1}\right)$, the relative $(\%)$ increase in $\mathrm{EF}$ should be much higher than the percentage increase in relative HR. This was confirmed by the overestimation of EE during the higher intensity. The EE/HR-relationship determined during the lower intensity overestimated individual EE during the higher intensity and vice versa. The change in regression equation of EE over HR during the higher intensity consisted of an increase in the slope of the regression line and a decrease in regression $y$-intercept (see figure 7.4). Because the regression lines intersect at a heart rate outside the range of exercise heart rates (in fact at a non-biological 249 beats. $\left.\mathrm{min}^{-1}\right)$, the decrease in $y$-intercept reflects an increase in relative heart rate over the complete range of exercise heart rates. The increase in regression slope indicates that this shift in heart rate is somewhat larger at lower heart rates. What could cause this change in EE/HR-relationship?

Previous research, comparing leg and arm work, revealed that arm work or combined arm/leg work and/or intermittent exercise elicits a higher HR relative to 
energy demand of leg exercise $(3,4,6,10)$. Since weight training consists of arm as well as leg work, a relative larger increase in arm work during a weight-training session would increase relative HR. From figure 7.1 it is obvious that the arm exercise (exercise number $1,3,5,6,8$ and 10) was associated with the lower EE and HR's. Therefore, a shift in relative HR due to increased arm exercise would only explain a shift in relative $\mathrm{HR}$ at lower levels of EE and $\mathrm{HR}$. The design of the training sessions, however, did not change (50\% of the available time spend for upper body and $50 \% \mathrm{leg}$ work), so it is not likely that a change in the relative contribution of arm work could explain the overall shift in relative $\mathrm{HR}$.

Some investigators suggest that the higher HR during weight training (combined arm and leg work) compared to $100 \%$ leg exercise may be due, at least in part, to elevated sympathetic activity $(1,5,6)$. This elevated sympathetic activity can be caused by sustained static exercise, performance of the Valsalva maneuver, or/and by a stimulation of chemosensitive afferent fibers by decreased muscle cell pH. Furthermore, during weight training, the combined static/dynamic muscle activity elevates intra-muscular pressure in combination with elevated intra-thoracic and intra-abdominal pressures causing restricted venous return $(1,7)$. To maintain cardiac output, heart rate should increase. Since the intensity of the training is higher during session $\mathrm{Hl}$ and $\mathrm{H} 2$, the effect of the above-mentioned factors on relative $\mathrm{HR}$ will also increase.

The shift in relative HR is most pronounced at lower HR and EE values. These lower values were not only associated with arm exercise but also for a large part with the resting/recovery periods. This enlarged effect during the resting periods denotes that the EE/HR-relationship is not only affected during exercise (i.e., compensation of the reduced venous return) but also due to factors that affect the resting/recovery periods (i.e., associate with an increased sympathetic output).

Accuracy. Previous research on EE/HR calibration curves to estimate combined arm and leg work, revealed levels of accuracy that ranged from 10 to $56 \%(6,8,11,13$, 14). Like in the present study, most of these accuracies included a systematic error. Estimations of EE were most accurate in studies that used calibration protocols that closely resembled the physical activity of interest (13). In the present study the difference between predicted and measured $\mathrm{EE}$ was $\approx 11 \%$ showing a better than average accuracy. However, although only the load changed whereas the movements (exercises), duration, pace and recovery time were exactly the same, there was still a systematic difference between the predicted and measured $\mathrm{EE}$. This finding is in concordance with the study of Maas (11) that revealed an increasing overestimation of oxygen uptake (up to $38 \%$ ) as load increased during a combined static/dynamic weight carrying task. Therefore, to avoid a systematic over/under-estimation, the determination of the EE/HR-relationship should take place at a heart rate or intensity close to expected exercise heart rates.

The determination of the EE/HR-relationship at two different intensities opens the possibility of calculating a correction factor (see Fig. 7.3). Because the increase in intensity consisted of standard weights, the increase in WT-intensity and therefore increase in HR $(1,9)$ differed substantially between the subjects (from 3.5 to 22.9 
beats $\left.\min ^{-1}\right)$. This wide range of changes in average HR was strongly correlated with the absolute $\left(\mathrm{kJ} \cdot \mathrm{min}^{-1}\right)$ inaccuracy. The arithmetical relationship between these two variables can subsequently be applied to correct predicted EE. Although this correction factor increased accuracy to $3.3 \%$ it is always preferable to determine an EE/HRrelationship that does not need to be corrected.

Drift in EE/HR-relationship during a weight-training session. The difference in average residual between the first and second half of a training session indicated that there was an effect of exercise duration on the EE/HR-relationship. During exercise the EE/HRrelationship drifted to a higher relative HR. This cardiovascular drift has also been observed during other modes of intermittent exercise (10). This indicates that besides a comparable intensity and mode of exercise also a comparable duration should be used to determine an accurate task specific EE/HR-relationship. Moreover, this drift in relative HR during both intensities furthermore confirms the previous assumption that the difference in warming up stress would not affect the EE/HR-relationship. The influence of a higher WT-intensity clearly exceeded a possible warming-up effect since the gradual drift in relative $\mathrm{HR}$ was not reduced but became even larger during sessions $\mathrm{H} 1$ and $\mathrm{H} 2$. This was displayed by a larger difference in average residuals between the first and second half of a single session.

In conclusion, this study described the accuracy of an EE/HR-relationship determined with a non-incremental intermittent calibration protocol. The results revealed that this protocol yields an EE/HR-relationship that predicts EE with a high level of accuracy. This accuracy will further increase as similar exercise and calibration intensities and durations are used.

\section{REFERENCES}

1 Collins, M. A., K. J. Cureton, D. W. Hill, C. A. Ray. Relationship of heart rate to oxygen uptake during weight lifting exercise. Med. Sci. Sports Exerc. 23(5):636640, 1991.

2 Franklin, B. A. Exercise testing, training and arm ergometry. Sports Med. 2:100$119,1985$.

3 Garbutt, G., M. G. Boocock, J. D. G. Troup. Physiological and spinal responses to circuit weight-training. Ergonomics 37(1):117-125, 1994.

4 Hempel, L. S., C. L. Wells. Cardiorespiratory cost of the Nautilus express circuit. Phys. Sportsmed. 13(4):82-97, 1985.

5 Hill, D. W., S. D. Butler. Haemodynamic responses to weightlifting exercise. Sports Med. 12(1):1-7, 1991.

6 Hurley, B. F., D. R. Seals, A. A. Ehsani, et al. Effects of high-intensity strength training on cardiovascular function. Med. Sci. Sports Exerc. 16(5):483-488, 1984. 
7 Keul, J., G. Haralambie, M. Bruder, H.-J. Gottstein. The effect of weight lifting on heart rate and metabolism in experienced weight lifters. Med. Sci. Sports Exerc. 10(1):13-15, 1978.

8 Li, R., P. Deurenberg, J. G. A. J. Hautvast. A critical evaluation of heart rate monitoring to assess energy expenditure in individuals. Am. J. Clin. Nutr. 58:602-607, 1993.

9 Londeree, B. R., T. R. Thomas, G. Ziogas, T. D. Smith, Q. Zhang. \%VO2max versus \%HR max regressions for six modes of exercise. Med. Sci. Sports Exerc. 27(3):458-461, 1995.

10 Lothian, F., M. R. Farrally. A comparison of methods for estimating oxygen uptake during intermittent exercise. Joumal of Sports Sciences 13:491-497, 1995.

11 Maas, S., M. L. J. Kok, H. G. Westra, H. C. G. Kemper. The validity of the use of heart rate in estimating oxygen consumption in static and in combined static/dynamic exercise. Ergonomics 32(2):141-148, 1989.

12 Murgatroyd, P. R., P. S. Shetty, A. M. Prentice. Techniques for the measurement of human energy expenditure: a practical guide. International Joumal of Obesity 17:549-568, 1993.

$13 \mathrm{Oja}, \mathrm{P} ., \mathrm{J}$. Ilmarinen, V. Louhevaara. Heart rate as an estimator of oxygen consumption during manual postal delivery. Scand. J. Work Environ. Health 8:2936, 1982.

14 Parker, S. B., B. F. Hurley, P. D. Hanlon, P. Vaccaro. Failure of target heart rate to accurately monitor intensity during aerobic dance. Med. Sci. Sports Exc. 21(2):230-234, 1989.

15 Pendergast, D. R. Cardiovascular, respiratory, and metabolic responses to upper body exercise. Med. Sci. Sports Exerc. 21(5):s121-s125, 1989.

16 Sawaka, M. N., D. S. Miles, J. S. Petrofsky, S. W. Wilde, R. M. Glaser, Physiology of upper body exercise, in Exercise and Sports Sciences Reviews, vol. 14, Pandolf, K. B., Ed. New York: Macmillan, 1986, pp. 175-211.

17 Weir de, J. B. New methods for calculating metabolic rate with special reference to protein metabolism. J. Physiol. 109:1-9, 1949. 


\section{Chapter 8}

\section{Effect of an 18-week weight-training program on energy expenditure and physical activity}

Ludo M.L.A. van Etten, Klaas R. Westerterp, Frans T.J. Verstappen ${ }^{\star}$, Bart J.B. Boon, and Wim H.M. Saris.

Department of Human Biology and *Department of Movement Sciences, Maastricht University, Maastricht, The Netherlands.

J. Appl. Physiol. 82(1): In Press, 1997

\section{ABSTRACT}

The purpose of this study was to examine the effect of an 18 wk weight-training program on average daily metabolic rate (ADMR). Before the intervention, in wk 8 and wk $18\left(T_{0}, T_{8}\right.$, and $\left.T_{18}\right)$ data on body composition, sleeping metabolic rate (SMR), food intake, energy cost of the weight-training program (EEex) and non-training physical activity (accelerometer) were collected in the exercise group (EXER, $n=18$ males). ADMR was determined in a subgroup $(E X 12, n=12)$ by using doubly labeled

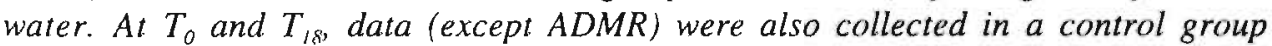
(CONT, n=8). Body mass did not change in EXER nor CONT. Fat-free mass increased only in EXER with $2.1 \pm 1.2 \mathrm{~kg}$, whereas fat mass decreased in EXER as well as CONT $\left(2.0 \pm 1.8\right.$ and $1.4 \pm 1.0 \mathrm{~kg}$, respectively). Initial ADMR $\left(12.4 \pm 1.2 \mathrm{MJ} \cdot\right.$ day $\left.^{-1}\right)$ increased at $T_{s}\left(13.5 \pm 1.3 \mathrm{MJ} \cdot \mathrm{day}^{-7}, P<0.001\right)$ with no further increase at $T_{78}(13.5$ $\pm 1.9 \mathrm{MJ} \cdot$ day $\left.^{-1}\right)$. SMR did not change in EXER $\left(4.8 \pm 0.5,4.9 \pm 0.5,4.8 \pm 0.5 \mathrm{~kJ} \cdot \mathrm{min}^{-1}\right)$ nor CONT $\left(4.7 \pm 0.4,4.8 \pm 0.4 \mathrm{~kJ} \cdot \mathrm{min}^{-1}\right)$. Energy intake did not change in EXER $(10.1$ $\left.\pm 1.8,9.7 \pm 1.8,9.2 \pm 1.9 \mathrm{MJ} \cdot \mathrm{day}^{-1}\right)$ or CONT $\left(10.2 \pm 2.6,9.4 \pm 1.8,10.1 \pm 1.5 \mathrm{MJ} \cdot \mathrm{day}^{-1}\right)$ and was systematically underreported in EXI2 $(-21 \pm 14,-28 \pm 18,-34 \pm 14 \%$,

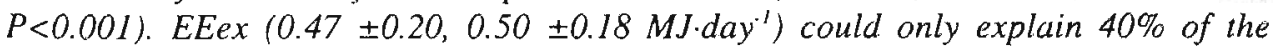
increase in ADMR. Nontraining physical activity did not change in both groups. In conclusion, although of modest energy cost, weight training induc's a significint increase in ADMR. 


\section{INTRODUCTION}

Evidence for an important role of increased physical activity in the quality of life and the primary prevention of coronary heart disease and cancer has grown in recent years $(1,2,7)$. For the majority of the people having a sedentary job, the recommended raise in general physical activity can be achieved by exercise, sport, recreation and life-style activitics. Depending on the field of interest and available time, a choice can be made from an extensive selection of difterent sports activities. Weight training is an example of an individual sport that is not restricted by time of the day or weather conditions and most forms of weight training are reported to be safe, even for hypertensive and cardiac patients and the elderly $(15,16)$. With the introduction of the easy-to-use weight stack machines and electronic ergometers applied during warming-up and cooling-down exercises (treadmills, bicycles, rower and step machines), the popularity of weight training increased. Research on the effect of weight training on health and fitness determinants revealed that weight training, like other types of exercise, positively affects physical performance and body composition and a number of health parameters $(21,23,27,30)$. Almost every study revealed an increase in muscular strength whereas the effect on aerobic power is inconsistent and depending on the type of weight training (e.g., circuit versus heavy resistance training (28), high versus low volume weight training (27)). Compared with running and cycling, the weighttraining-induced changes in body composition consist of a larger increase in fat-frec mass whereas the decrease in fat mass seems to be somewhat smaller. The modest effect on fat mass might be attributed to the lower energy costs of a single weighttraining workout (17). The latter finding seems to make this kind of exercise less effective in programs of weight control and weight reduction (17). However, previous studies suggested that the effect of physical exercise on average daily metabolic rate (ADMR) exceeds the energy cost of the training-work itself. This finding initiated research on the effect of exercise on other components of ADMR like the thermic effect of feeding (22) and sleeping/resting metabolic rate (32), or on excess postexercise energy expenditure (20). Studies that measured ADMR confirmed that the energy demand of the added physical exercise explains only partly the increase in $\operatorname{ADMR}(5,11,35)$. A validated method to measure ADMR in free living subjects is the doubly labeled water method $\left({ }^{2} \mathrm{H}_{2}{ }^{18} \mathrm{O}\right)$. Due to the high cost only a few studies used this technique. Until now, no study investigated the effect of weight training on ADMR.

The purpose of this study was to investigate the effect of an 18 wk weight-training program on ADMR and the components sleeping metabolic rate and the energy cost of physical activity (nontraining and exercise activity) to quantify their contribution to an ensuing change in ADMR. A 3-day food record was used to estimate energy intake and food composition. A triaxial accelerometer was used to register nontraining physical activity. 
$\downarrow T_{0} \quad \downarrow T_{8} \quad \downarrow T_{18}$

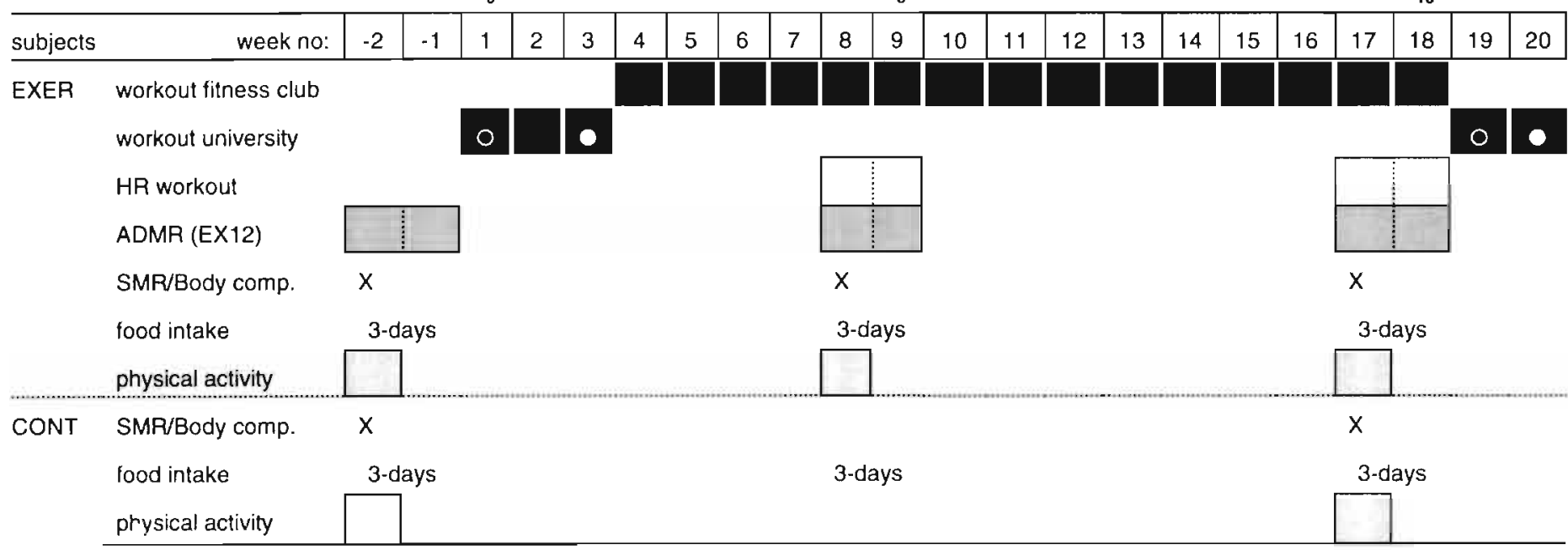

Fig. 8.1. Overall design. The actual training program consisted of 18 wk of weight training( $)$. The firs 3 wk were performed at the university to determine the relationship between energy expenditure and heart rate in week 3 (d). Week 19 and 20 were not considered as part of the training program but were used to check it the energy expenditure-heart rate relationship was changed. During weeks 1 and 19 an incremental cycling test was performed (e). ADMR, average daily metabolic rate; SMR, sleeping metabolic rate; EXER, exercise group; CONT, control group. 


\section{METHODS}

The overall design of the study is presented in Fig. 8.1.

Subjects. Twenty-six healthy sedentary males were selected to participate in the study. None of the subjects had engaged in a regular exercise program for at least two yrs hufore the study. Detailed information concerning the purpose and all methods used in the study was provided, and written consent was obtained. Eighteen subjects participated in the exercise group (EXER) and eight subjects served as inactive controls (CONT).

Average Daily Metabolic Rate (ADMR). ADMR was measured over a period of $2 \mathrm{wk}$, using the doubly labeled water $\left({ }^{2} \mathrm{H}_{2}{ }^{18} \mathrm{O}\right)$ method according to the Maastricht protocol (37). Due to the high cost of this technique, ADMR was only measured in 12 subjects (EX12) randomly selected from the exercise group. The ADMR measurement was blinded by giving all subjects an isotope drink; the selected 12 subjects recieved ${ }^{2} \mathrm{H}_{2}{ }^{18} \mathrm{O}$, and the remaining subjects recieved ${ }^{2} \mathrm{H}_{2} \mathrm{O}$ for body composition measurements only (see below). The sampling medium for measuring the isotope enrichment and disappearance was urine. After a background urine sample was collected, the isotope dose was administered as the last consumption before the night ( 22:30). Urine samples from the second (8:00-10:00) and last (21:00-23:00) voiding of the day were collected at days 1,8 and 14 (in all, 1 background +6 enriched samples). Samples were analysed with isotope ratio mass spectrometry (Aqua Sira, VG, UK).

Sleeping metabolic rate (SMR). SMR was measured during an overnight stay in a respiration chamber (18.00-8.00). Dinner was eaten before entering the chamber, and subjects were not allowed to eat again until body composition was determined the next morning. To minimize the residual effects of training on energy expenditure, subjects were asked to refrain from any exercise or strenuous activity on the day before the measurement. Hence, SMR measurement was always $>8 \mathrm{~h}$ after the last meal and $>30$ $\mathrm{h}$ after the last training session. More technical information on the respiration chamber is described elsewhere (12). SMR was defined as the lowest mean energy expenditure over a shiftable 3-h interval between 0:00-6:00.

Physical characteristics. Physical characteristics are shown in Table 8.1. Anthropometrical measurements were taken the morning after the controlled stay at the laboratory. After subjects had voided, body mass was measured to an accuracy of $5 \mathrm{~g}$ on an electronic scale (Mettler, E1200). Body volume was determined by using the hydrostatic weighing technique. Residual lung volume was simultaneously measured by using the helium dilution technique (Volugraph 200, Mijnhart). Total body water $(T B W)$ was measured using deuterium $\left({ }^{2} \mathrm{H}_{2} \mathrm{O}\right)$ dilution. Body composition was assessed by using the three-component model of Siri that combines body density with TBW (13). 
Table 8.1. Characteristics for the exercise (EXER, $n=18$ ) and control group (CONT, $n=8$ ).

\begin{tabular}{lccccc}
\hline & \multicolumn{2}{c}{ EXER } & \multicolumn{2}{c}{ CONT } \\
\cline { 2 - 3 } \cline { 5 - 6 } age $(\mathrm{yr})$ & $33 \pm 6$ & $23-41$ & & $35 \pm 6$ & $24-41$ \\
height $(\mathrm{m})$ & $1.82 \pm 0.07$ & $1.71-1.97$ & & $1.82 \pm 0.05$ & $1.75-1.88$ \\
body mass $(\mathrm{kg})$ & $78.8 \pm 9.7$ & $64.4-103.1$ & $78.0 \pm 10.8$ & $56.1-88.8$ \\
fat mass $(\mathrm{kg})$ & $19.1 \pm 4.7$ & $13.7-31.2$ & & $17.7 \pm 6.1$ & $6.4-26.1$ \\
fat-free mass $(\mathrm{kg})$ & $59.7 \pm 6.6$ & $49.2-71.9$ & $60.3 \pm 7.2$ & $49.7-69.9$ \\
fat $(\%)$ & $24 \pm 4$ & $17-30$ & & $22 \pm 6$ & $11-29$ \\
\hline
\end{tabular}

Values are mean $\pm \mathrm{SD}$ or ranges. Analysis revealed no differences between groups.

Nontraining physical activity. Physical activity over a 7-day period was registered by using a triaxial accelerometer consisting of three uniaxial piezoresistive accelerometers (8). The accelerometer was attached to the low back with the use of an elastic belt. The accelerometer was connected to a portable data unit for on-line processing and storage of acceleration data. The data logger was programmed to calculate the sum of the integrated acceleration curves from all three directions. The time period for integration was set at one min and physical activity was expressed as counts.min ${ }^{-1}$. Subjects were instructed to wear the accelerometer during waking hours, except during bathing and workouts at the fitness club. Furthermore, subjects were asked to record the periods they traveled by vehicles for periods $>15 \mathrm{~min}$. These periods were excluded from analysis. Furthermore, all data with a value above 8000 counts-min ${ }^{-1}$ were excluded because they were assumed not to be produced by human movement (9).

Food intake. To estimate food intake and food composition, a 3-day food record (including 1 weekend day) was used. After completion, the food record was checked with the subject to clarify the records and eliminate inconsistencies.

Training program. The subjects trained two times a week on non-consecutive days for $18 \mathrm{wk}$. The first $3 \mathrm{wk}$, the training sessions were performed at the university (see below). The workouts at the fitness club consisted of 10 exercises using weight stack machines (Sportesse, Germany) and free weights. Three sets of 15 repetitions were performed on the following exercises: bench press, fly's, squat, leg curl, leg extension, seated rowing, lat pull down, dumbbell curl, triceps push down and sit ups. Additionally, each workout included a 10-min warming up and cooling-down of cycling on a self selected intensity. All workouts were supervised by a fitness instructor.

Energy cost of weight training. Energy expenditure during the workouts at the fitness center (EEex) was predicted from exercise heart rate (HR). Because the relationship 
between energy expenditure and heart rate (EE/HR-relationship) is different for weight-training exercises and leg cycling, two separate equations were used.

To determine the EE/HR-relationship during weight training, the first 3 wk of the weight-training program were performed at the university (total of 6 sessions). The computer-paced sessions consisted of the following exercises: bench press, lunges, dumbbell pull, squat, and the overhead lats pulley ( $3 \times 15$ repetitions). This sequence of five exercises was completed twice to increase the volume of the session without increasing the number of different exercises. Duration was $42 \mathrm{~min}$, whereas exercise time was equally split between upper body and leg exercise. Work-to-rest ratio was $1: 1$. Besides these 10 exercises, the session consisted of a 10-min warming up and cooling down, being cycling at 100 or $150 \mathrm{~W}$, depending on the subjects Wmax $(<70 \% \mathrm{Wmax}$ ). The first 2 weeks (i.e., first 4 sessions) were used to practice the techniques and to get accommodated to the laboratory setting and computerized pacing. During the two sessions in week 3, gas-exchange (Oxycon Beta, Mijnhart) and heart rate (Polar Sport Tester model PE3000) were continuously measured. Gasexchange was converted to EE with the use of the Weir formula (33). Data of both sessions were pooled before calculating the linear regression equation of $\mathrm{EE}$ over $\mathrm{HR}$.

To determine the EE/HR-relationship during cycling, a stepwise incremental cycling test was performed in week 1 . The test started with 4 min of cycling at $50 \mathrm{~W}$. Subsequently, load was increased every 3 min with respectively $50 \mathrm{~W}$ if $\mathrm{HR}<160$ beats $\min ^{-1}$ and $25 \mathrm{~W}$ if $\mathrm{HR} \geq 160$ beats $\mathrm{min}^{-1}$, until exhaustion.

To check whether the training program induced a change in the EE/HR-relationship (pre-intervention equation), the calibration protocol was repeated after the 18 wk of training (postintervention equation). However, three instead of four sessions were used for accommodation.

Statistical analysis. The analysis of variance (ANOVA) for repeated measures was used to compare means, including the two groups as a between-subject factor. Between-group initial values were compared using a simple factorial ANOVA. Statistical significance was set at $\mathrm{P}<0.05$.

\section{RESULTS}

Where appropriate, the results are presented for the entire exercise group (EXER) as well as the subgroup (EX12) in which ADMR was measured.

Changes in physical characteristics. As shown in Table 8.2, after 18 weeks both the EXER and CONT had lost a comparable amount of fat mass $(2.0 \pm 1.8 \mathrm{~kg}: \mathrm{P}<0.001$ and $1.4 \pm 1.0 \mathrm{~kg} ; \mathrm{P}<0.05$, respectively) whereas fat-free mass increased only in EXER $(2.1 \pm 1.2 \mathrm{~kg} ; \mathrm{P}<0.001$ and $0.4 \pm 1.8 \mathrm{~kg}$; NS, respectively). The combined changes in fat mass and lat-free mass resulted in an unaltered body mass $(0.1 \pm 1.5 \mathrm{~kg}$; NS and $1.0 \pm 1.2 \mathrm{~kg}$; NS, respectively) but a similar decrease in percentage fat of $-2.6 \pm 2.0 \%$; 
$\mathrm{P}<0.001$ and $-1.6 \pm 1.5 \% ; \mathrm{P}<0.05$, respectively. In EX12, body mass increased at $\mathrm{T}_{8}$ with $1.1 \pm 1.5 \mathrm{~kg}(\mathrm{P}<0.05)$ returning to pre-intervention values at $\mathrm{T}_{18}$.

Table 8.2. Changes in body composition in the exercise (EXER), and the control group (CONT).

\begin{tabular}{lccc}
\hline & \multicolumn{2}{c}{ EXER } & CONT \\
\cline { 2 - 4 } & $\mathrm{T}_{8}$ & $\mathrm{~T}_{18}$ & $\mathrm{~T}_{18}$ \\
body mass $(\mathrm{kg})$ & $0.4 \pm 2.2$ & $0.1 \pm 1.5$ & $-1.0 \pm 1.2$ \\
fat mass $(\mathrm{kg})$ & $-0.8 \pm 1.8$ & $-2.0 \pm 1.8^{* *}$ & $-1.4 \pm 1.0^{*}$ \\
fat-free mass $(\mathrm{kg})$ & $1.3 \pm 1.3^{* *}$ & $2.1 \pm 1.2^{* * \neq}$ & $0.4 \pm 1.8^{q}$ \\
fat $(\%)$ & $-1.3 \pm 1.7^{*}$ & $-2.6 \pm 2.0^{* * \ddagger}$ & $-1.6 \pm 1.5^{*}$ \\
\hline
\end{tabular}

significantly different from $T_{0}: \cdot P<0.05, " P<0.001$ and different from $T_{-}:: P<0.05$ significantly different between the groups : ${ }^{1} \mathrm{P}<0.01$

Average daily metabolic rate (ADMR, $n=12$ ). At $\mathrm{T}_{8}$ the initial ADMR increased from $12.4 \pm 1.2 \mathrm{MJ} \cdot$ day $^{-1}$ to $13.5 \pm 1.3 \mathrm{MJ} \cdot$ day $^{-1}(9.3 \%, \mathrm{P}<0.001)$. At $\mathrm{T}_{18}, \mathrm{ADMR}$ was 13.5 $\pm 1.9 \mathrm{MJ} \cdot$ day $^{-1}$ revealing no further increase. The mean relative increase in ADMR over the 18 wk period was $9.5 \%$.

Sleeping metabolic rate (SMR). There were no changes in SMR in EXER $\left(\mathrm{T}_{0}, \mathrm{~T}_{8}\right.$, and $\left.\mathrm{T}_{1 \mathrm{x}}: 4.8 \pm 0.5,4.9 \pm 0.5,4.8 \pm 0.5 \mathrm{~kJ} \cdot \mathrm{min}^{-1}\right)$ and $\operatorname{CONT}\left(\mathrm{T}_{0}, \mathrm{~T}_{18}: 4.7 \pm 0.4,4.8 \pm 0.4\right.$ $\left.\mathrm{kJ} \cdot \mathrm{min}^{-1}\right)$. In EX12, however, SMR increased significantly at $\mathrm{T}_{8}(\mathrm{P}<0.05)$, but at $\mathrm{T}_{18}$ SMR did not differ from $\mathrm{T}_{0}$ or $\mathrm{T}_{8}\left(4.6 \pm 0.4,4.9 \pm 0.4,4.7 \pm 0.3 \mathrm{~kJ} \cdot \mathrm{min}^{-1}\right.$, respectively). The increase in SMR at $\mathrm{T}_{\varepsilon}$ was not correlated with changes in body mass or fat-free mass $(\mathrm{P}=0.14)$.

Food intake. As shown in Fig. 8.2, average daily energy intake did not differ between $\mathrm{T}_{0}, \mathrm{~T}_{8}$ and $\mathrm{T}_{18}$ in EXER $\left(10.1 \pm 1.8,9.7 \pm 1.8,9.2 \pm 1.9 \mathrm{MJ} \cdot\right.$ day $\left.^{-1}\right)$ or in CONT (10.2 $\pm 2.6,9.4 \pm 1.8,10.1 \pm 1.5 \mathrm{MJ} \cdot$ day $^{-1}$, respectively). Food composition did not differ between the groups at the start of the study and did not change during the study. The average $(n=26)$ contribution to daily energy intake of the macronutrients: carbohydrate, fat, protein and alcohol was $48 \pm 7,35 \pm 7,15 \pm 3$ and $4 \pm 4 \%$, respectively. In EX12, energy intake was significant lower than ADMR at all three moments of data collection $\left(-2.6 \pm 1.8,-3.9 \pm 2.6,-4.6 \pm 2.0 \mathrm{MJ} \cdot \mathrm{day}^{-1}, \mathrm{P}<0.001\right)$. This underreporting in absolute and relative terms $(-21 \pm 14,-28 \pm 18,-34 \pm 14 \%)$, increased during the study but was only significantly different between $T_{0}$ and $T_{18}(P<0.01)$.

Workout at the fitness club. Average training compliance, including the six training sessions in week $1-3$, was $95 \pm 7 \%$. The average number and duration of the workouts did not differ between $T_{8}$ and $T_{18}\left(1.9 \pm 0.5\right.$ vs $1.9 \pm 0.5$ sessions. week ${ }^{-1}, 71 \pm 11$ vs 73 \pm 13 min $\cdot$ workout $^{-1}$ ). To check whether the EE/HR-relationship was affected by the 


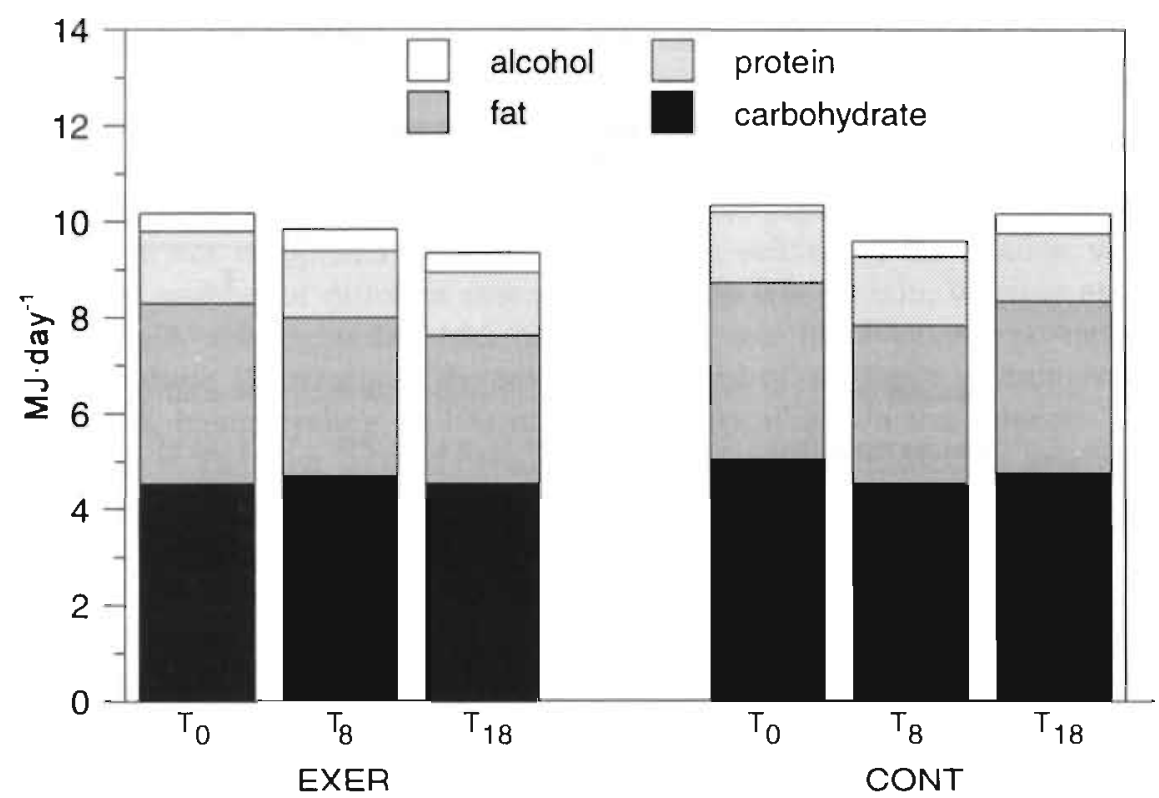

Fig. 8.2. Energy and macronuirient intake as measured with a 3-day dietary record before (Td), and after 8 and 18 wk weight training $\left(T_{8}\right.$, and $T_{18}$, respectively) for the entire exercise group (EXER), and the control group (CONT).

training program, both pre- and postintervention equations were used to predict EEex at $T_{8}$ and $T_{18}$. Preintervention equations always resulted in a significantly lower EEex compared with post-intervention equations, revealing a change in the exercise EE/HRrelationship (at $\mathrm{T}_{8}: 28.7 \pm 5.6$ vs $32.7 \pm 6.5 \mathrm{~kJ} \cdot \mathrm{min}^{-1}$; at $\mathrm{T}_{18}: 26.7 \pm 5.1$ vs $30.8 \pm 5.4$ $\left.\mathrm{kJ} \cdot \mathrm{min}^{-1}, \mathrm{P}<0.00 \mathrm{I}\right)$. Hence, the pre-intervention equations were used to estimate EEex at $T_{8}$, whereas postintervention equations were applied at $T_{18}$ Average EEex increased significantly in EXER $\left(28.7 \pm 5.6\right.$ vs $30.8 \pm 5.4 \mathrm{~kJ} \cdot \mathrm{min}^{-1}$ equal to $2014 \pm 446$ vs 2271 $\pm 607 \mathrm{~kJ} \cdot$ session $^{-1}, \mathrm{P}<0.05$ ) but did not change in EXI2 ( $29.8 \pm 4.9$ vs $30.9 \pm 6.0$ $\mathrm{kJ} \cdot \min ^{-1}$ equal to $1952 \pm 348$ vs $2151 \pm 616 \mathrm{~kJ}$-session $\left.{ }^{-1}\right)$. Net daily energy cost of the workouts (EEex-SMR, MJ.day ${ }^{-1}$ ) was not different between $\mathrm{T}_{8}$ and $\mathrm{T}_{18}$ in EXER $(0.47$

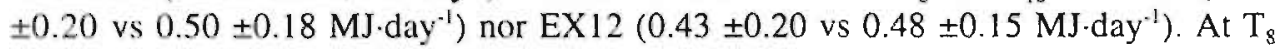
and $\mathrm{T}_{18}$, only $38 \%$ and $41 \%$ of the increase in ADMR could be attributed to the net energy cost of the workouts performed at the fitness club.

The components of ADMR. The following components of ADMR (see Fig. 8.3) were calculated: 24-h SMR (MJ.day ${ }^{-1}$ ), diet-induced thermogenesis (DIT) and the energy cost of physical activity (ADMR-SMR-DIT, MJ.day ${ }^{-1}$ ). The latter was subsequently split up into the net cost of weight training and the cost of non-training physical activity. The DIT was assumed be $10 \%$ of the measured ADMR. The energy cost of physical activity increased significantly $(\mathrm{P}<0.01)$ at $\mathrm{T}_{8}$ but showed no further increase at $\mathrm{T}_{18}\left(4.4 \pm 0.9,5.3 \pm 0.9,5.5 \pm 1.6 \mathrm{MJ} \cdot\right.$ day $^{-1}$, respectively $)$. However, the energy cost of 
the non-training physical activity (ADMR-DIT-SMR-net EEex) did not change (4.4 $\pm 0.9,4.9 \pm 0.8,5.0 \pm 1.6 \mathrm{MJ} \cdot$ day $^{-1}$, respectively).

Non-training physical activity. As depicted in Fig. 8.4, physical activity as measured with the triaxial accelerometer did not change between $T_{0}, T_{8}$ and $T_{18}$ in the exercise group $\left(967 \pm 158,1052 \pm 214,1068 \pm 249\right.$ counts $\cdot \mathrm{min}^{-1}$ ) or the control group (1119 $\pm 222,1137 \pm 311$ counts $\left.\min ^{-1}\right)$. Also, activity time did not change in both groups (EXER: $14.0 \pm 1.7,12.9 \pm 2.5,13.3 \pm 2.3 \mathrm{~h} \cdot \mathrm{day}^{-1}$ and CONT: $14.3 \pm 1.1,12.7 \pm 2.3$ $h \cdot$ day $\left.^{-1}\right)$. Average weekly variation in accelerometer output $(n=26)$, expressed as coefficient of variation (individual $\mathrm{SD} / \overline{\mathrm{x}} \times 100$ ) was $14 \pm 8 \%$. The daily coefficient of variation decreased gradually during the study and was significantly different between $\mathrm{T}_{0}$ and $\mathrm{T}_{18}(28.0 \pm 9.9$, vs $20.5 \pm 10.3 \%, \mathrm{P}<0.01)$.

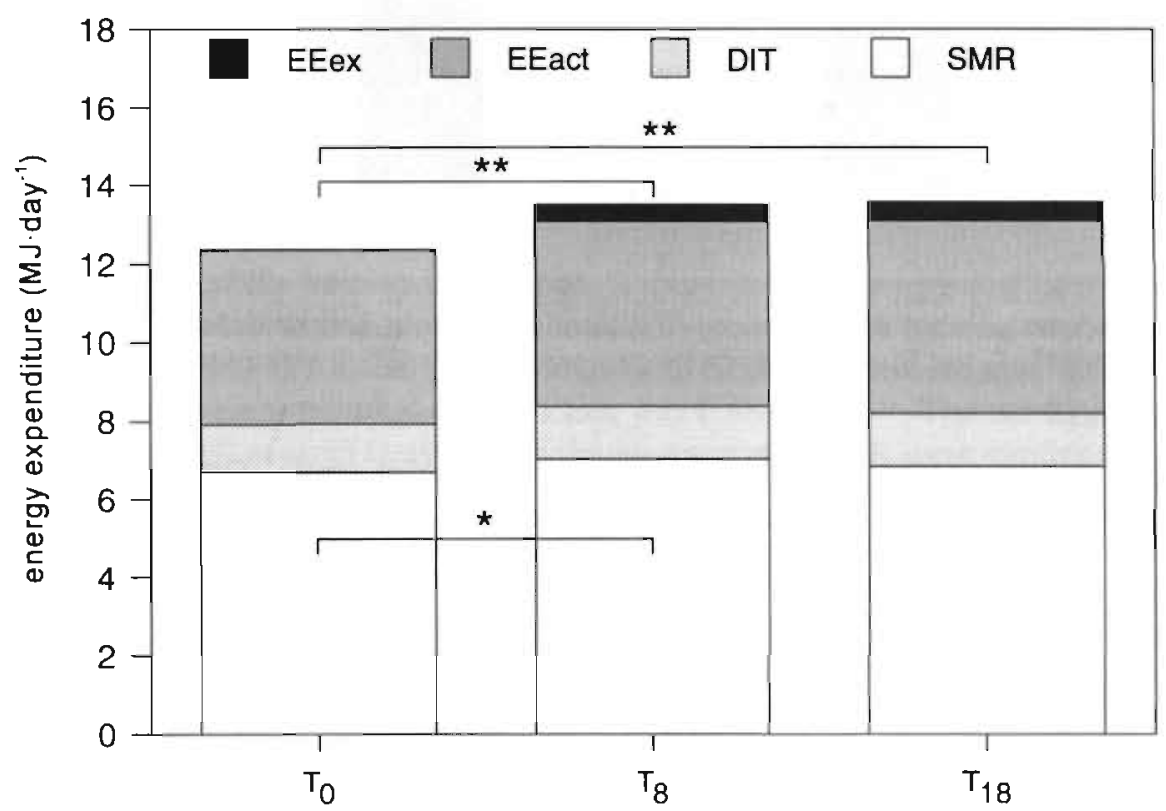

Fig. 8.3. Mean values of $A D M R$ ( $n=12)$ and its components: 24 -h sleeping metabolic rate (SMR), diet-induced thermogenesis (DIT), net energy cost of the exercise (EEex=EE workout-SMR) and energy expenditure for nontraining physical activity (EEact=ADMR-SMR-DIT-EEex), before and after 8 and 18 wk of weight training $\left(T_{o} T_{8}\right.$ and $T_{i,}$, respectively); " $P<0.05$, " $P<0.001$.

\section{DISCUSSION}

Changes in physical characteristics. Although the change in fat mass in the exercise group is in concordance with weight-training-induced changes in fat mass found in the literature $\left(-0.1 \mathrm{~kg} \cdot\right.$ week $\left.^{-1}\right)(38)$, the change in fat mass in the control group is an indication that seasonal changes in food habits or spontaneous activity, although not 


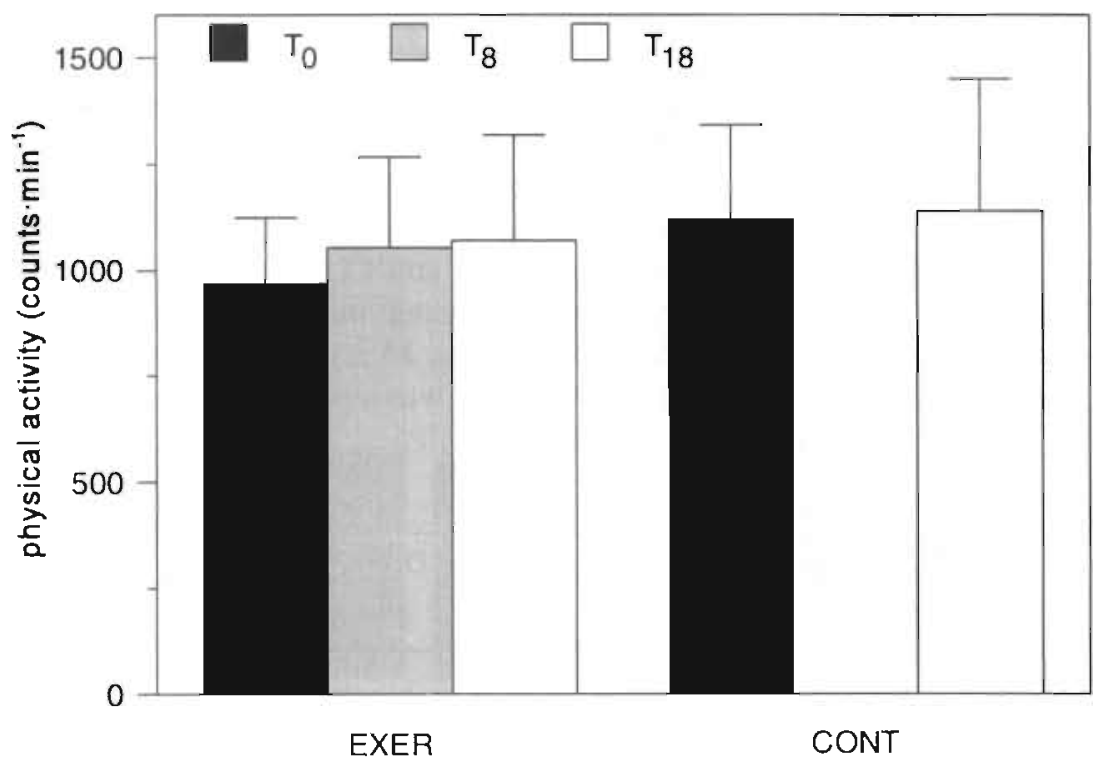

Fig. 8.4. Nontraining physical activity measured with a triaxial accelerometer, before and after 8 and 18 wk of weight training $\left(T_{0,} T_{8}\right.$, and $T_{18}$, respectively) for the entire exercise group (EXER) and controls (CONT; $T_{0}$ and $T_{18}$.

detected by the accelerometer output, could have played a role. The increase in fatfree mass was most likely due to the exercise because no change was found in the controls. Assuming an energy equivalent of 38.9 and $6.3 \mathrm{MJ} \cdot \mathrm{kg}^{-1}$ fat mass and fat-free mass, respectively (34), it can be calculated that the subjects in both the EXER-and CONT-group were in a small negative energy balance over the total period of eighteen weeks (0.5 and $0.4 \mathrm{MJ} \cdot$ day $^{-1}$, respectively).

ADMR. This study examined the effect of an 18-wk weight-training program on average daily energy expenditure and found a $1.1{\mathrm{MJ} \cdot d a y^{-1}}^{-19.3 \%)}$ and $1.2 \mathrm{MJ} \cdot \mathrm{day}^{-1}(9.5 \%)$ increase after 8 and $18 \mathrm{wk}$, respectively. To our knowledge, only four other studies used doubly labeled water to measure the effect of an exercise program on ADMR. The interventions, however, consisted of endurance-training instead of weight training. Meijer et al. (19) studied the effect of 20 weeks endurance-training in a group of 32 untrained subjects preparing to run a half marathon. ADMR was determined in a subgroup of 4 males and 3 females. After 8 and $20 \mathrm{wk}$, ADMR was significantly increased with $\sim 1.6$ and $2.2 \mathrm{MJ} \cdot \mathrm{day}^{-1}$ (15 and $20 \%$, respectively). To examine the effect over a prolonged period. Westerterp et al. (35) measured ADMR in a subgroup of 13 subjects that continued the previous mentioned training program for another 20 weeks, and found no further increase in ADMR after 40 weeks (2.3 MJ.day ${ }^{-1}, 21 \%$ ). Bingham et al. (4) studied a group of three males and two females that followed a 
9-wk running program ( $60 \mathrm{~min}, 5$ day-week ${ }^{-1}$ ) and found an average increase of 2.8 MJ.day ${ }^{-1}(28 \%)$. Blaak et al. (5) found an average increase of $1.3 \mathrm{MJ} \cdot$ day $^{-1}(21 \%)$ in a group of 10 obese boys, ages $10-11 \mathrm{yr}$, following a cycling program $(4 \mathrm{wk}, 5$ sessions. $\mathrm{wk}^{-1}, 45 \mathrm{~min}$ cycling at $50-60 \% \mathrm{VO} 2 \mathrm{max}$ ). In contrast with the above mentioned studies that found an exercise induced increase in ADMR, a study of Goran and Poehlman (14) revealed no change in ADMR in a group of 11 elderly subjects that followed an 8-wk cycling program.

Campbell et al. (11) examined the effect of weight training on ADMR, but this study did not use the doubly labeled water technique. The required energy intake to maintain body weight was used to estimate ADMR in a study on the effect of a 12-wk weight-training program in older adults $(n=12)$. An average $1.1 \mathrm{MJ}^{-}$day ${ }^{-1}(\approx 15 \%)$ increase in daily energy intake was required to compensate the cost of weight-training exercise and the increase in resting metabolic rate.

Because the above-mentioned interventions differed not only in the mode of activity but also varied widely in intensity. duration and frequency of the training program, additional information on the average net energy cost of the extra exercise and changes in the remaining components of ADMR is required to compare and interpret the effect of the various training programs on ADMR.

Net energy cost of the training intervention. Because the net energy cost of a training program depends on the intensity, duration and frequency of the training sessions, it is evident that studies can differ considerable in exercise-induced changes in ADMR. After 18 wk of weight training, the net EEex was $0.48 \mathrm{MJ} \cdot$ day $^{-11}$. The net EEex in the study of Campbell et al. (11) as well as the increase in ADMR were similar with the present study ( 0.42 and $1.1 \mathrm{MJ}^{-}$day $^{-1}$, respectively). Although the energy cost of the training program in the study of Blaak et al. (5) and Goran and Poehlman (14) was $-50 \%$ higher $\left( \pm 0.63 \mathrm{MJ} \cdot\right.$ day $\left.^{-1}\right)$ compared with the present study, ADMR did only increase in the study of Blaak et al. (5) that used a group of young boys (1.3 MJ.day $\left.{ }^{-1}\right)$. In the elderly group, ADMR did not change. This was explained by a $60 \%$ reduction in spontaneous and/or voluntary physical activity. The net EEex of the added exercise in the study of Meijer et al. (19) was almost twice as high. The increase in ADMR in the latter study was also almost twice the increase of the present study $(0.87$ and 2.2 $\mathrm{MJ} \cdot$ day $^{-1}$, respectively). In the paper of Bingham et al. (4) no values on net EEex were presented. From calculations based on the average time ( $37 \mathrm{~min})$, speed $\left(11.5 \mathrm{~km} \cdot \mathrm{h}^{-1}\right)$ and body mass $(63 \mathrm{~kg})$, net EEex was estimated to be $1.9 \mathrm{MJ} \cdot$ day $^{-1}(18)$. This higher EEex also induced a larger increase in $\operatorname{ADMR}\left(2.8 \mathrm{MJ} \cdot \mathrm{day}^{-1}\right)$.

In general, interventions with higher EEex showed a higher increase in ADMR. The increase in ADMR, however, always exceeded the energy expenditure due to exercise. As shown in the study by Goran and Poehlman (14), exercise could also affect the remaining components of ADMR (i.e., spontaneous activity). Therefore, other components of ADMR like sleeping metabolic rate and nontraining (spontaneous) physical activity should always be included in the design. 
Sleeping metabolic rate. Studies on the effect of exercise on SMR are somewhat controversial (22). The unchanged SMR in the entire exercise-group $(n=18)$ over a period of $18 \mathrm{wk}$ was in concordance with a previous weight-training intervention that did not reveal a change in SMR after 12 wk of weight training (32). In EX 12, however, SMR showed a small increase at $\mathrm{T}_{8}\left(0.25 \pm 0.27 \mathrm{~kJ} \cdot \mathrm{min}^{-1}, 5 \%\right)$, but returned to pre-intervention values after $18 \mathrm{wk}$ of training. Body mass and fat-free mass, two important determinants of SMR, also increased at $T_{8}$. The change in SMR, however, could not be explained by the increase in body mass or fat-free mass at $T_{8}$, probably due the small changes in SMR and the anthropometric data.

Two of the previous mentioned studies $(11,14)$, both using elderly subjects, reported a change in resting metabolic rate, whereas the present study and the three remaining studies $(4,5,19)$ showed no change in SMR. Besides differences in average age, intervention, changes in body mass, and level of training, the protocol used to determine resting energy expenditure (SMR versus resting metabolic rate) might affect the outcome of the study. In some studies that measured both SMR and resting metabolic rate after a weight-training intervention (31) or a bout of endurance exercise (3), resting energy expenditure increased whereas sleeping energy expenditure did not change. On the other hand, Bingham (4) measured also both parameters but found no change in resting or sleeping metabolic rate.

Differences in training intervention (type of exercise) are not likely to explain the inconsistency in exercise induced changes in SMR. Cross-sectional (30) as well as intervention studies (10) that compared the effect of either high-intensity resistance or endurance training, revealed no exercise-specific change in resting metabolic rate.

Although some studies reveal a substantial training induced increase in resting metabolic rate (up to 10\%), the increasing effect on ADMR would be at the most $5 \%$.

Dietary intake. A 3-day food record was considered to provide information on the food quotient (FQ). The FQ was supposed to reflect the respiratory exchange ratio required to calculate oxygen consumption during the doubly labeled water period. Unfortunately, the results of the 3-day food record revealed a substantial, and gradually increasing underreporting. Although underreporting is also found in other studies (25, 29,36 ), the increasing magnitude of the underreporting made the data unusable for the calculation of DIT and FQ. Since the changes in body composition revealed only a small negative energy balance, DIT was set at $10 \%$ of the measured ADMR (26). An average food quotient of 0.85 was assumed $(6,35)$ during the three measurement periods, because changes in FQ will be relatively small and therefore will not greatly affect the calculation of ADMR (39).

Energy cost of physical activity. The net energy cost (ADMR-SMR-DIT) of physical activity increased significantly by 0.9 and $1.1 \mathrm{MJ}$-day ${ }^{-1}$, equivalent to 20 and $25 \%$. Like other studies that revealed an increase in $\operatorname{ADMR}(5,11,19)$, the net EEex explains only partly $( \pm 40-50 \%)$ the increase in the net energy cost of physical activity. In the present study and the study of Meijer et al. (19), the remaining discrepancy could not be attributed to a change in nontraining activity as recorded by an 
accelerometer. The unchanged accelerometer output in the present study is in line with the unchanged net energy cost of physical activity at $T_{8}$ and $T_{18}$. Also Blaak et al. (5) found no change in spontaneous activity measured by heart rate recording. As indicated by the large weekly and daily coefficients of variation it is possible, although not likely, that the accelerometer method is not sensible enough to measure small changes in physical activity. The decreased daily variation in physical activity at the end of the study was probably due to a seasonal and/or weather changes (study started in winter and ended in summer).

Because the energy cost of physical activity is not directly measured but the residue of subtracting SMR, DIT and net EEex from ADMR, the discrepancy could also be due to measurement errors in one of these components. Part of the discrepancy could be attributed to a slight underestimation of the energy cost of EEex because it does not include the residual energy expenditure associated with postexercise recovery or a short-term effect on SMR. However, the magnitude of postexercise recovery is assumed to be small following both endurance exercise (38-125 kJ per exercise bout (24)) as well as weight training $( \pm 80 \mathrm{~kJ}(20))$. This small contribution $(76-250 \mathrm{~kJ}$. week $^{-1}$ ) of postexercise recovery to the net energy cost of exercise can hardly explain the remaining part of the increased energy expenditure (a total of $4.4 \mathrm{MJ} \cdot \mathrm{week}^{-1}$ ). On the other hand, a small underestimation of SMR due to a short-term effect (SMR was measured $>30 \mathrm{~h}$ postexercise), could largely explain the unaccountable increase in energy cost of physical activity and the unchanged physical activity. Although the effect of weight training on the separate components of ADMR remains partially unclear, for the determination of the efficacy of weight training in weight-control programs, the absolute change in ADMR is the main value of interest.

In summary. The unchanged accelerometer output revealed that there was no change in (nontraining) physical activity in both the exercise as well as the control group. Hence, we assume that the change in daily energy expenditure could be attributed to the weight-training program. Therefore, a weight-training program that consisted of two sessions.week ${ }^{-1}$ resulted in an average increase in daily energy expenditure of approximately $10 \%$ after $8 \mathrm{wk}$, with no further increase after $18 \mathrm{wk}$. Only $40 \%$ of the increase could be attributed to the net energy cost of the program. The unexplained part of the increase in ADMR could be due to an underestimation of the net EEex or measurement errors in other components of ADMR, although the results on SMR revealed no significant change. Weight training did not change body mass, and there was a $2.1 \mathrm{~kg}$ increase in fat-free mass in the exercise group. The exercise group as well as the control group showed a decrease in fat mass. From the change in daily energy expenditure, it can be concluded that weight training, although of modest energy cost compared to endurance training, induces a significant increase in ADMR and therefore can be applied as an effective and save adjunct to exercise based weightcontrol programs. 


\section{REFERENCES}

1 Åstrand, P.-O. Why exercise? Med. Sci. Sports Exerc. 24(2):153-162, 1992.

2 Berlin, J. A., G. A. Colditz. A meta-analysis of physical activity in the prevention of coronary heart disease. Am. J. Epidemiol. 132(4):612-628, 1990.

3 Bielinski, R., Y. Schutz, E. Jéquir. Energy metabolism during the postexercise recovery in man. Am. J. Clin. Nutr. 42:69-82, 1985.

4 Bingham, S. A., G. R. Goldberg, W. A. Coward, J. H. Cummings. The effect of exercise and improved physical fitness on basal metabolic rate. Brit. J. Nutr. 61:155-173, 1989.

5 Blaak, E. E., K. R. Westerterp, O. Bar-Or, L. J. M. Wouters, W. H. M. Saris. Total energy expenditure and spontaneous activity in relation to training in obese boys. Am. J. Clin. Nutr. 55:777-782, 1992.

6 Black, A. E., A. M. Prentice, W. A. Coward. Use of food quotients to predict respiratory quotients for the double labelled water method of measuring energy expenditure. Hum. Nutr. Clin. Nutr. 40C:381-391, 1986.

7 Blair, S. N., H. W. Kohl, R. S. Paffenbarger, D. G. Clark, K. H. Cooper, L. W. Gibbons. Physical fitness and all-cause mortality. A prospective study on healthy men and women. JAMA 262(17):2395-2401. 1989.

8 Bouten, C. V., K. R. Westerterp, M. Verduin, J. D. Janssen. Assessment of energy expenditure for physical activity using a triaxial accelerometer. Med. Sci. Sports Exerc, 26(12):1516-1523, 1994.

9 Bouten, C. V. C., W. P. H. G. Verboeket-van de Venne, K. R. Westerterp, M. Verduin, J. D. Janssen. Daily physical activity assessment: comparison between movement registration and doubly labeled water. J. Appl. Physiol. 81(2):10191026, 1996.

10 Broeder, C. E., K. A. Burrhus, L. S. Svanevik, J. Wilmore. The effects of either high-intensity resistance or endurance training on resting metabolic rate. Am. $J$. Clin. Nutr. 55:802-810, 1992.

11 Campbell, W. W., M. C. Crim, V. R. Young, W. J. Evans. Increased energy requirements and changes in body composition with resistance training in older adults. Am. J. Clin. Nutr. 60:167-175, 1994.

12 Dale van, D., P. F. M. Schoffelen, F. Hoor ten. W. H. M. Saris. Effects of addition of exercise to energy restriction on 24-hour energy expenditure, sleeping metabolic rate and daily physical activity. Eur. J. Clin. Nutr. 43:441-451, 1989.

13 Fuller, N. J., S. A. Jebb, M. A. Laskey, W. A. Coward, M. Elia. Four-component model for the assessment of body composition in humans: comparison with alternative methods, and evaluation of the density and hydration of fat -free mass. Clin. Sci. 82:687-693, 1992.

14 Goran, M. I., E. T. Poehlman. Endurance training does not enhance total energy expenditure in healthy elderly persons. Am. J. Physiol. 263(26):E950-E957, 1992.

15 Hill, D. W., S. D. Butler. Haemodynamic responses to weightlifting exercise. Sports Med. 12(1):1-7, 1991. 
16 Hurley, B. F., R. A. Redmond, R. E. Pratley, M. S. Treuth, M. A. Rogers, A. P. Goldberg. Effects of strength training on muscle hypertrophy and muscle disruption in older men. Int. J. Sports Med. 16(6):378-384, 1995.

17 McArdle, W. D., G. F. Foglia. Energy cost and cardiorespiratory stress of isometric and weight training exercises. J. Sports Med. Phys. Fitness 9:23-30, 1969.

18 Medicine, A. C. o. S., Guidelines for exercise testing and prescription, 4th ed. Philadelphia: Lea \& Febiger, 1991.

19 Meijer, G. A. L., G. M. E. Janssen, K. R. Westerterp, F. Verhoeven, W. H. M. Saris, F. Hoor ten. The effect of a 5-month endurance-training programme on physical activity: evidence for sex-difference in the metabolic response to exercise. Eur. J. Appl. Physiol. 62:11-17, 1991.

20 Melby, C. L., T. Tincknell, W. D. Schmidt. Energy expenditure following a bout of non-steady state resistance exercise. J. Sports Med. Phys. Fitness 32:128-135, 1992.

21 Miller, W., W. Sherman, J. Ivy. Effect of strength training on glucose tolerance and post-glucose insulin response. Med. Sci. Sports Exerc. 16:539-543, 1984.

22 Poehlman, E. T. A review: exercise and its influence on resting energy metabolism in man. Med. Sci. Sports Exerc. 21(5):515-525, 1989.

23 Poehlman, E. T., A. W. Gardner, P. A. Ades, et al. Resting energy metabolism and cardiovascular disease risk in resistance-trained and aerobically trained males. Metabolism 41(12):1351-1360, 1992.

24 Poehlman, E. T., C. L. Melby, M. I. Goran. The impact of exercise and diet restriction on daily energy expenditure. Sports Med. 11:78-101, 1991.

25 Schoeller, D. A., C. R. Fjeld. Human energy metabolism: what have we learned from the doubly labeled water method? Ann. Rev. Nutr. 11:355-373, 1991.

26 Schutz, Y., T. Bessard, E. Jequier. Diet-induced thermogenesis measured over a whole day in obese and nonobese women. Am. J. Clin. Nutr. 40:542-552, 1984.

27 Stone, M. H., S. J. Fleck, N. Travis Triplet, W. J. Kraemer. Health and performance-related potential of resistance training. Sports Med. 11(4):210-231, 1991.

28 Stone, M. H., D. G. Wilson. Resistive training and selected effects. Med. Clin. N. Am. 69(1): 109-122, 1985.

29 Thompson, J. L., M. M. Manore, J. S. Skinner, E. Ravussin, M. Spraul. Daily energy expenditure in male endurance athletes with differing energy intakes. Med. Sci. Sports Exi'rc. 27(3):347-354, 1995.

30 Toth, M. T., E. T. Poehlman. Resting metabolic rate and cardiovascular disease risk in resistance- and aerobic-trained middle aged women. Int. J. Obesity 9:691698, 1995.

31 Treuth, M. S., G. R. Hunter, R. L. Weinsier, S. H. Kell. Energy expenditure and substrate utilization in older women after strength training: 24-h calorimeter results. J. Appl. Physiol. 78(6):2140-2146, 1995.

32 Van Etten, L. M. L. A., K. R. Westerterp, F. T. J. Verstappen. Effect of weighttraining on energy expenditure and substrate utilization during sleep. Med. Sci. Sports Exerc. 27(2):188-193, 1995. 
33 Weir de, J. B. New methods for calculating metabolic rate with special reference to protein metabolism. J. Physiol. 109:1-9, 1949.

34 Westerterp, K. R., Balance between energy intake and energy expenditure, in Food intake and energy expenditure, Westerterp-Plantenga, M. S., E. W. H. M. Fredrix, and A. B. Steffens, Eds. Boca Raton: CRC Press, 1994, pp. 291-309.

35 Westerterp, K. R., A. Meijer, E. Janssen, W. Saris, F. Hoor ten. Long term effect of physical activity on energy balance and body composition. Brit. J. Nutr. 68:2130, 1992.

36 Westerterp, K. R., W. P. H. G. Verboeket-van de Venne, G. A. L. Meijer, F. Hoor ten, Self-reporled intike as a measure for energy intake, a validation against doubly labelled water, in Obesity in Europe 91, Ailhaud, G., B. Guy-Grand, M. Lafontan, and D. Ricquier, Eds. London: John Libbey \& Company, 1992. pp. 1722.

37 Westerterp, K. R., L. Wouters, W. D. Marken Lichtenbelt. The Maastricht protocol for the measurement of body composition and energy expenditure with labeled water. Obesity Research 3(suppl 1):49-57, 1995.

38 Wilmore, J. H. Variations in physical activity habits and body composition. Int. J. Obesity 19(sup 4):S107-S112, 1995.

39 Wolfe, R. R., Measurement of total energy expenditure using the doubly-labeled water method, in Radioactive and stable isotope tracers in biomedicine: principles and practice of kinetic analysis. New York: Wiley-Liss, Inc, 1992, pp. 207-233. 


\section{Chapter 9}

\section{General discussion}

The research presented in this thesis focuses on the effects of weight training exercisc on energy expenditure, body composition and physical performance. Adding a physical exercise program to the usual activity regime is the most obvious alternative to increase daily energy expenditure (ADMR). In general, studies on the effect of exercise on ADMR revealed an increase in ADMR. However, an exercise induced increase in ADMR is not as evident as it appears. The extra energy cost of the added exercise could be compensated by a decreased spontaneous physical activity (5), or diminished by a decreased energy cost of physical exercise due to a higher training status (12). Until now only endurance modes of activity (e.g., running and cycling) were used to increase physical activity. Elaborating on the findings of these endurance type of interventions, this thesis focuses on the effectiveness of an interval mode of activity (i.e., weight training) as a tool to increase ADMR. To investigate whether possible side-effects on sleeping metabolic rate (SMR), energy cost of exercise or spontanenus physical activity, bias the exercise-induced effect on ADMR information on these variables were also collected.

Body composition (\%fat) and physical performance were included because their interrelation with energy expenditure and because of their practical relevance for subjects participating in a weight-training program. Exercise can affect \%fat by changing fat mass and/or fat-free mass. Fat mass is affected through the energy balance equation. Depending on the initial discrepancy between energy intake and energy expenditure, the increased ADMR will shift the energy balance towards a negative or a less positive balance. An increase in fat-free mass is a well documented adaptation to an increased physical activity. Data on muscular strength and aerobic capacity were included as effect-parameters for physical performance.

This chapter integrates and discusses the results from the different studies and summarizes successively the results of the various studies on: ADMR, SMR, EE during exercise, body composition, and physical performance.

\section{CHANGES IN ADMR AND ITS COMPONENTS}

Performing a weight-training program that consisted of two approximately $70 \mathrm{~min}$ workouts a week, resulted in an increase in ADMR of approximately $10 \%$ in a group of originally sedentary men (chapter 8 ). However, only $40-50 \%$ of the increase in 
ADMR could be attributed to the exercise program whereas data on SMR as measured in a respiration chamber and physical activity as measured by an accelerometer revealed no change. It was concluded that the unexplained increase in ADMR (4.4 $\mathrm{MJ} \cdot \mathrm{wk}^{-1}$ ) could be due to an underestimation of the energy cost of the weight-training program, a short-term increase in SMR and/or a non-detected increase in spontaneous physical activity. The following paragraphs discus to what extent an underestimation in one or more of these components could account for the unexplained increase in ADMR by integrating findings from various chapters.

Estimated energy cost of the workouts (EEex). The two major causes of an incorrect estimation of EEex are a limited validity of the heart ritte monitoring method used to estimate EEex, and the omission of the post-exercise energy expenditure (i.e., recovery energy expenditure or Excess Post Exercise Energy Expenditure in analogy with Excess Post Exercise Oxygen Consumption) in the calculation of the energy cost of the workouts.

* Validity of the heart rate monitoring method. Prior to examining the effect of weight training on ADMR (study-C), a study was performed to validate the method of predicting EEex using heart rate monitoring (study-B) (18). This experimental group consisted of well-trained subjects with previous weight-training experience. The intervention study, however, would consist of sedentary males. Therefore, the analysis of study-B was repeated including also the untrained subjects from study-C. Both validation studies revealed that energy expenditure during weight training could be predicted accurately provided that the energy expenditure-heart rate relationship is determined at a comparable exercise intensity (i.e., comparable heart rate). A higher intensity during the workout would result in an overestimated predicted EEex and vice versa a lower exercise heart rate in an underestimated EEex. In the ADMR study, heart rate during the calibration protocol versus workout at $T_{8}$ and $T_{18}$ was $128 \pm 15$ versus $121 \pm 11$ and $112 \pm 15$ versus $118 \pm 8$ beats. $\mathrm{min}^{-1}$, respectively (chapter 6 ). Since workout-HR at $T_{x}$ was lower than HR during calibration EEex might be underestimated whereas EEcx at $T_{18}$ would more likely be overestimated. Based on the relationship between the extrapolated (i.e., predicted) and measured EEex as determined in chapter 7 (se' Fig. 7.4), the difference in HR during the calibration protocol and workouts would result in a calculated prediction error of $\pm 3 \%$ or $\pm 0.1 \mathrm{MJ} \cdot \mathrm{wk}^{-1}$. Based on these data it is not likely that the unexplained part of the increase in ADMR can be ascribed to an error in the prediction of EEex. It is even more likely that EEex was slightly overestimated. However. the validation studies were performed under optimal conditions using exactly the same exercises, pace, work/rest-ratio and a standard 50\% difference in training loads between the calibration sessions and the predicted sessions. During the workouts at the fitness club these variables differed largely between subjects and workouts. Therefore, it still remains possible that differences in loads, pace, work/rest-ratio and type of exercises between the calibration sessions and the workout session affected the accuracy of the heart rate monitoring method. 
* Magnitude of recovery energy expenditure (Excess Post Exercise Energy Expenditure). Previous research already revealed that the magnitude of recovery energy expenditure following a bout of moderately intense weight-training exercises is small (9). To verify this finding and to examine the effect of training status (untrained versus trained) on recovery energy expenditure (see chapter 6. Fig. 6.5), data on recovery energy expenditure were collected during study-C. Recovery energy expenditure was defined as the difference between a $15 \mathrm{~min}$ pre-session and a $15 \mathrm{~min}$ post-session resting period. The data on recovery energy expenditure following two standard exercise sessions using two different loads (i.e., lower and higher intensity) revealed that:

- post-session energy expenditure returned to pre-session values within the $15 \mathrm{~min}$ recovery period during both exercise intensities;

- a higher exercise intensity (i.e., heart rate) resulted in a higher post-exercise energy expenditure $(27.8 \pm 9.0$ versus $48.3 \pm 16.9 \mathrm{~kJ}$ ) (see Fig. 9.1).

- recovery energy expenditure was approximately $2 \%$ of the total energy cost of the performed exercise;

- total excess energy expenditure decreased after $18 \mathrm{wk}$ of weight training for the lower $(19.8 \pm 10.6 \mathrm{~kJ})$ and higher $(33.1 \pm 10.6 \mathrm{~kJ})$ weight-training intensity.

Comparing exercise heart rates during the standardized calibration sessions in the laboratory and the workouts at the fitness club revealed that the latter were somewhat

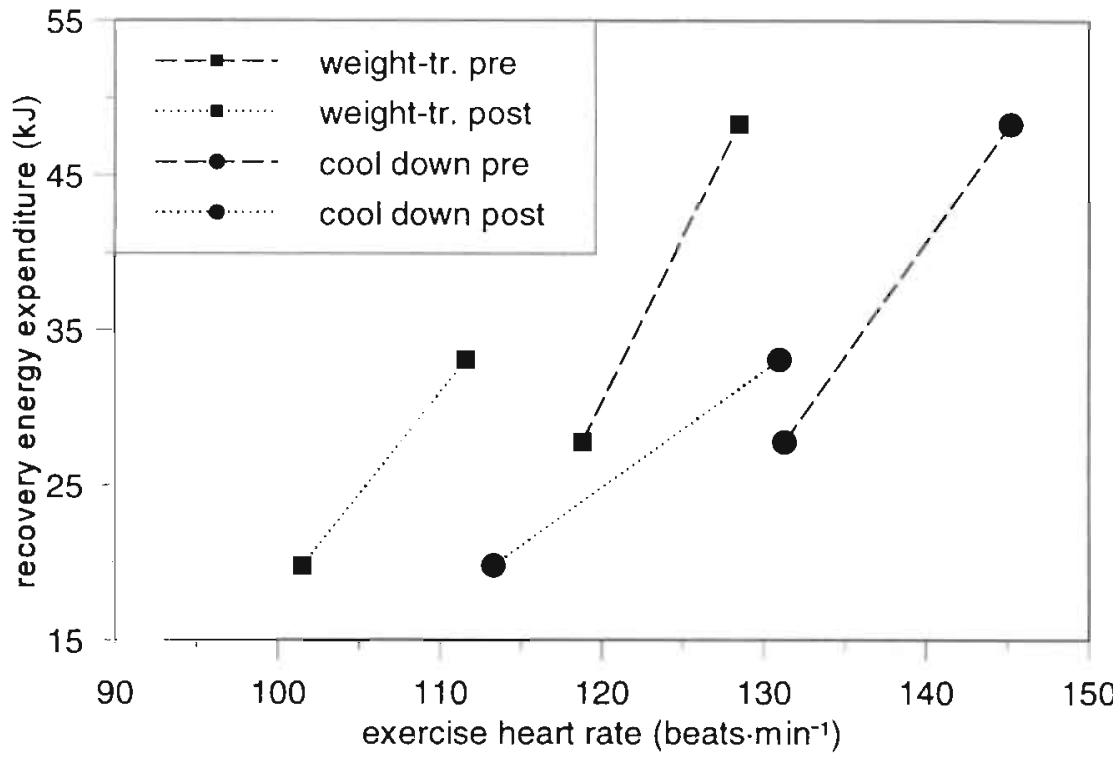

Fig.9.1. Pre- and post-intervention recovery energy expenditure plotted against average exercise heart rates during the lower and higher weight-training phase (weight-tr.), and during the cooling down phase (cool down) pertorming arm cranking and cycling. 
higher (see chapter 6, Table 6.3 and 6.5). Particularly when taken into account that workouts at the fitness club consisted of the same number of exercises whereas the duration was $10 \mathrm{~min}$ longer. This increased duration is most likely due to longer resting/recovery periods between the exercises. Longer resting periods will decrease average exercise heart rate. Hence, if comparable work/rest-ratios were used, the difference between the average heart rate during laboratory session and the workouts would even be more conclusive. Since exercise intensity is positively related to the magnitude of recovery energy expenditure, the excess post exercise energy expenditure during the workouts at the fitness club will also be somewhat higher. However, it is still not likely that a substantial part of the unexplained increase in ADMR can be ascribed to the exclusion of recovery energy expenditure when calculating EEex.

Sleeping metabolic rate. Sleeping metabolic rate is the second mentioned factor that could account for the unexplained increase in ADMR. Because SMR is the largest component of ADMR, a relatively small weight-training-induced raise in SMR will substantially increase daily energy expenditure. Therefore, the two intervention studies (study-A and study-B, chapter 3 and 8 , respectively) focussed on a possible weight-training-induced effect on SMR.

To avoid a residual short term effect of exercise on SMR, the measurements were scheduled at least $30 \mathrm{~h}$ after the last training bout. Consequently, no information was obtained on a possible short-term effect. Both intervention studies revealed no long term effect on SMR. Based on the measured SMR (47.8 MJ.wk $\left.{ }^{-1}\right)$, and the unexplained increase in ADMR (4.4 MJ.w $\left.\mathrm{K}^{-1}\right)$ in study-C, one might speculate on the effect of a changed SMR. If SMR was underestimated systematically 7 days $\cdot w^{-1}$, the required change to meet the unexplained increase in ADMR should be $4.4 / 0.478$ $\mathrm{MJ} \cdot \mathrm{wk}^{-1}=9.2 \%$. Because both intervention studies revealed no long term effect of weight training, an underestimated SMR is restricted to the $30 \mathrm{~h}$ post-exercise (2 times $\cdot \mathrm{wk}^{-1}=0.36 \mathrm{wk}$ ). To explain completely the remaining increase in ADMR, there had to be an implausible short term increase in SMR of $25.5 \%(=4.4 /(0.478 * 0.36)$ ). Exercise induce increases in SMR reach only up to $10 \%(11,15)$. A short term increase of this magnitude would result in an increase of $1.71 \mathrm{MJ} \cdot \mathrm{wk}^{-1}$ which accounts for $39 \%$ of the unexplained increase in ADMR. Although, an underestimation of SMR can explain a substantial part of the unexplained increase in ADMR, the larger part remains unexplained.

The energy cost of non-training physical activity (=ADMR-SMR-DIT-net EEex). Next to SMR, the energy cost of non-training (spontaneous) physical activity is the second important component of ADMR. The initial energy cost of non-training physical activity was $4.4 \mathrm{MJ}$-day ${ }^{-1}$ or $30.8 \mathrm{MJ} \cdot \mathrm{wk}^{-1}$. The partly explained increase in ADMR suggests that there had to be an increase in one or more of the remaining components of ADMR. Although the calculated non-training physical activity tended to increase at $\mathrm{T}_{\overline{5}}$ and $\mathrm{T}_{18}$ (4.9 and 5.0 MJ.day ${ }^{-1}$, respectively), they did not reach statistical significance $(\mathrm{P}=0.084$ and $\mathrm{P}=0.093$, respectively). However, one should keep in mind 
that the energy cost of non-training physical activity was calculated, and not directly measured.

Measurement of spontaneous physical activity. Since the energy costs of physical activity is related with the amount and intensity of activity, a change in physical activity could be used as an indirect measure of a change in the energy cost of spontaneous physical activity. A change in physical activity can be realized by a change in intensity and/or duration of the activity. A triaxial accelerometer was used to collect information on intensity and duration of physical activity. Intensity was estimated by the average accelerometer output whereas duration of physical activity was assumed to be reflected by the number of monitoring hours.

- intensity of physical activity. Findings of two validation studies of Bouten et al $(2,3)$ revealed that, although there was no significant relationship between accelerometer output and ADMR in a group of 30 free-living subjects obviously due to interindividual differences in body weight and SMR (2), the within subject correlation was highly significant during daily activities of moderate intensity (correlation coefficient $0.95 \pm 0.02$ ) (3). Therefore it is assumed that the unchanged average accelerometer output reflects an unchanged activity intensity.

- monitoring hours. With respect to a possible change in activity time (i.e., duration), analysis revealed no change in activity time from $T_{0}$ to $T_{18}(14.0,12.9$ and 13.3 $\mathrm{h} \cdot \mathrm{day}^{-1}$, respectively). The number of monitoring hours is equal to the average 13.6 \pm 1.4 monitoring hours found in the free-living validation study of Bouten et al (2).

In view of the reliability of the accelerometer and because the consistency between the unchanged accelerometer data and the calculated unchanged energy cost of physical activity, it is not likely that non-training physical activity substantially changed during the study.

In conclusion. Although not considered to be impossible, it is not likely that the unexplained increase can completely be ascribed to an underestimation in just EEex, SMR or the energy cost of physical activity. Probably, all components contribute to a different extent to the unexplained increase in ADMR.

\section{EXERCISE ECONOMY AND ENERGY COST OF PHYSICAL ACTIVITY}

As hypothesized in the introduction, physical training potentially has an opposing effect on energy expenditure. The extra work performed during the training sessions increases energy expenditure whereas an increase in training status decreases the energy cost of physical exercise (improves exercise economy). To what extent an improved exercise economy will also affect the energy cost of spontaneous activity is unknown.

The average decrease in energy cost of intermittent and continuous types of exercise was approximately $8.5 \%$ (see chapter 6 ). A comparable decrease in the energy cost of initial spontaneous activity $\left(4.4 \mathrm{MJ} \cdot \mathrm{day}^{-1}\right)$ would have resulted in an average 
decrease of $0.37 \mathrm{MJ} \cdot$ day $^{-1}$. This decrease would have compensated approximately $80 \%$ of the extra cost of the weight-training workouts $\left(0.48 \mathrm{MJ} \cdot\right.$ day $\left.^{-1}\right)$. Since the increase in ADMR even exceeded the energy cost of the weight-training program, it can be concluded that the potential opposing effect of an improved exercise economy does not compensate the extra energy cost of the added training program.

\section{CHANGES IN BODY COMPOSITION}

Although quantification of the weight-training-induced effect on daily energy expenditure was a major argument to initiate a weight-training intervention, for many people the primary motivation to increase ADMR is to bring about a change in \% fat. Based on the simple energy balance model, an increase in ADMR could result in a negative energy balance. Body fat reserve (i.e., fat mass) is an important energy source to meet this exercise-induced additional energy need.

Besides an exercise decrease in fat mass, exercise also affects fat-free mass. Exercise is known to increase fat-free mass in non-dieting subjects and to reduce the relative decrease in fat-free mass due to dietary restriction $(1,7)$. As demonstrated in chapter 2 , the exercise induced change in fat-free mass is also a function of initial body build. The measurement of body composition includes the measurement of fat mass as well as fat-free mass. To determine body composition several methods can be applied.

Methodological considerations. In this thesis a two compartment model (i.e., hydrostatic weighing) as well as a three compartment model (i.e., fat, water and the remaining fat-free dry mass) $(4,13)$ were used to determine body composition. An essential difference between these methods is that the two compartment model assumes a constant density of fat-free mass. It has been suggested that the density of fat-free mass in trained subjects may be affected by training and therefore could differ from the density found in the average population. If a training program or a change in physical activity (i.e., more active lifestyle) actually affect fat-free mass density, the assumption of a constant fat-free mass would result in a measurement error.

Because of the opposing effect of the relative contribution of muscle mass and bone mass on the density of fat-free mass it has been difficult to predict whether values would be higher or lower than assumed (e.g., $1.1 \mathrm{~g} \cdot \mathrm{ml}^{-1}$ by Siri (16)). A disproportional increase in muscle mass would tend to decrease density whereas a disproportional increase in bone mass would have the opposite effect. Recently, Modlesky et al (10) compared fat-free mass density between subjects with a weight-training-induced high musculoskeletal development and a group of non-weight-training controls with average musculoskeletal development. They found lower fat-free mass density in the weight-trainers compared to the controls due to a higher water and lower mineral and protein fraction. The lower fat-free mass density would result in an underestimation of fat-free mass and therefore an overestimated percentage of body fat. To what extent a weight-training intervention of 12-18 weeks will affect fat-free mass density is diffi- 
cult to predict. Since bone mass is not likely to change over such a short period, the change in fat-free mass will mainly consist of water and protein. Consequently, a weight-training-induced change in fat-free mass density would lead to an underestimation of the increase in fat-free mass and decrease in fat mass. Therefore, it is preferred to use a three compartment model in exercise intervention studies although the method is more expensive and time consuming compared to hydrostatic weighing.

Body build an body composition in the various studies. A major purpose of the first intervention study (study-A) was to examine the effect of body build -defined as the quantity of fat-free mass corrected for fat mass and height- on weight-training-induced changes in body composition and performance (chapter 2). It was concluded that the increase in fat-free mass is modified by initial body build. The group with a solid build increased fat-free mass whereas the slender group did not show a significant change in fat-free mass at all. In contrast with fat-free mass, both groups decreased fat mass. Accordingly, all participants became a more solid body build.

Because of this modifying effect on body composition, body build of the experimental populations from study-B and study-C were also calculated. Figure 9.2 depicts the body build of the participants of all three studies (A, B, and C) described in this thesis.

A weight-training-induced trend towards a more solid body build is corroborated by the body build of the trained subjects from study-B ( $\star$, see chapter 4 ). Body build of seven out of ten subjects fell above the borderline for solid build whereas the three remaining subjects would have been categorized having 'normal' body build (i.e., between dotted borderlines Fig. 9.2A). Compared with the subjects from the original body build-study $(\boldsymbol{\Lambda})$, the solid build of the trained subjects should merely be ascribed to a low fat mass index and not to a relative high fat-free mass index.

Body build of the experimental population participating in study-C (I, chapter 5 and 7) showed a slight trend to a more slender build (only three subjects fell within the solid build criteria, see Fig. 9.2). This trend to a more slender build was mainly a result of differences in inclusion criteria between both intervention studies. Study-A selected subjects on immoderate/extreme body build whereas study-C only included subjects with a Body Mass Index ranging from 20 to $25 \mathrm{~kg} \cdot \mathrm{m}^{-2}$ (on average $25.8 \pm 3.7$ versus $23.7 \pm 2.1 \mathrm{~kg} \cdot \mathrm{m}^{-2}, \mathrm{P}<0.05$ ). This distinction in design excluded subjects with solid body builds because this group exhibits in general a higher Body Mass Index (on average $28.8 \pm 2.1 \mathrm{~kg} \cdot \mathrm{m}^{-2}$ in study-A).

To what extent body build affected the weight-training-induced change in body composition in study-C is difficult to verify. Besides a difference in initial body build, also the duration of the intervention differed between the two intervention studies. Therefore, when comparing data on body composition from the intervention sludy- $\mathrm{A}$ and study-C, differences in duration and body build should be taken into account. It is assumed that there was no difference in seasonal effect on body composition since both studies started in the winter and ended in the summer period.

Although study-C lasted $50 \%$ longer there was no difference in the absolute $(-2.0$ \pm 1.8 versus $-2.1 \pm 1.7 \mathrm{~kg}$, respectively) or relative $(-10.7 \pm 9.9$ versus $-11.5 \pm 7.8 \%$, re- 

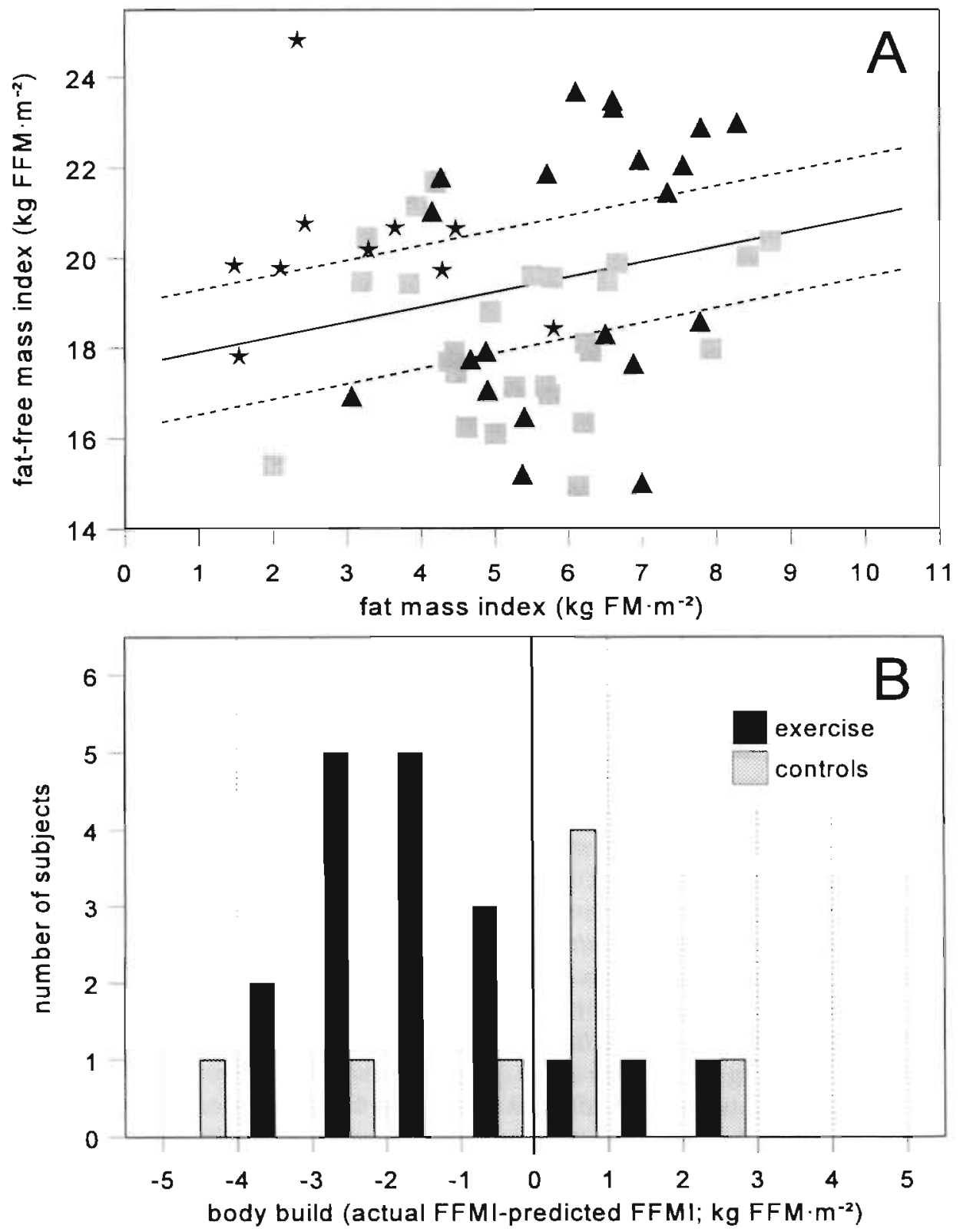

Fig. 9.2. A: Fat-free mass index (FFMI) plotted as a function of fat mass index (FMI) of three different experimental populations: the slender and solid population described in chapter 2 and 3 (A); the trained population described in chapter $4(\star)$; and the untrained population described in chapter 6 and 8() ). The histogram in Fig. $B$ depicts the trequency distribution of the body build of the subjects described in chapter 6 and 8 . 
spectively) decrease in fat mass between the two intervention studies. The average increase in fat-free mass in study-C, however, exceeded the average change in fat-free mass of study- $\mathrm{A}(2.1 \pm 1.2$ versus $1.1 \pm 1.3 \mathrm{~kg}, \mathrm{P}<0.05)$. Although the somewhat less beneficial initial body build in study- $\mathrm{C}$ with regard to changes in fat-free mass, the average increase in fat-free mass was even similar to the increase in the solid group from study-A (2.1 versus $1.6 \mathrm{~kg})$. The latter can be due to the $50 \%$ longer exercise program that provided a longer exercise stimulus as well as extended time to adapt. A longer time to adapt is important if adaptation speed was the main reason of the observed difference between the slender and the solid group from study-A. To what extent a difference in the energy cost or composition of the training program contributed to the findings on body composition is difficult to estimate. The training method did not show large differences since both exercise programs consisted of an all out effort during the third set. With regard to the total energy cost of the workouts it is difficult to speculate because the workouts in study-A were of a longer duration but used lower loads during the warming up and cooling down.

\section{CHANGES IN PHYSICAL PERFORMANCE}

Aerobic capacity and muscular strength were included as parameter to determine the weight-training-induced effect on physical performance. The most interesting effect on physical performance was the increase in aerobic capacity found in study-C (chapter 6). For two reasons this change in aerobic capacity was interesting. Firstly because a weight-training-induced increase in aerobic capacity is rarely found in conventional weight-training interventions. In general, weight-training-induced increases in aerobic capacity are restricted to circuit weight-training interventions (17). Secondly this increase was interesting because of the conflicting effect of weight training on aerobic capacity found between the two weight-training intervention studies. Data on aerobic capacity from both studies are presented in Table 9.1.

The mechanism by which weight training, especially noncircuit weight training, can increase VO2max is unclear (17). Besides a true central and/or peripheral aerobic effect, an increase in muscular strength or muscle girth might be capable of increasing aerobic capacity (6). Indeed, muscle strength during the leg extension increased with $8.4 \%$ which could have had an effect on aerobic capacity. On the other hand, a comparable $8.8 \%$ increase in isokinetic strength found in the first intervention study was not accompanied by an increase in $\mathrm{VO} 2 \max$. This inconsistency is certainly no protocol artefact because both studies used the same cycling and strength testing protocols. Furthermore, the absence of a VO2max increase in the control group of study-C supports a real intervention-induced effect (see chapter 6 ).

Contrary to a strength-induced-increase in aerobic capacity, the increase in $\mathrm{VO} 2 \mathrm{max}$ can also be due to pre-intervention muscle weakness. In a review on exercise testing, Noakes (14) concluded that not only muscle oxidative capacity but also muscle power determines maximal aerobic performance. Therefore, pre-intervention VO2max can be underestimated due to muscle weakness. Consequently, an exercise-induced-increase 
in lower body strength would not really increase VO2max but would allow peak cycle ergometry VO2, to become a closer expression of the true $\mathrm{VO} 2 \mathrm{max}$. In this case, a preand post intervention VO2max difference does not reflect a true increase in aerobic capacity. The absence of a pre- and post-intervention difference in maximal heart rate and respiratory exchange ratio (RER, see Table 9.1), however, does not confirm a possible insufficient power output that could have limited subjects to attain true VO2max during pre-intervention testing.

Table 9.1. Data on maximal aerobic power, oxygen consumption, heart rate and respiratory quotient during the incremental cycling tests of the first and second weight-training intervention (study- $A$ and study-C, respectively)

\begin{tabular}{|c|c|c|c|c|c|}
\hline & study & $T_{0}$ & $T_{B}$ & $T_{12}$ & $T_{18}$ \\
\hline VO2max & A & $3.10 \pm 0.47$ & - & $3.07 \pm 0.35$ & \\
\hline$\left(1 \cdot \min ^{-1}\right)$ & C & $3.08 \pm 0.57$ & $3.47 \pm 0.52^{* *}$ & - & $3.62 \pm 0.61^{\star *}$ \\
\hline $\mathrm{ml} \cdot \mathrm{kg}^{-1} \cdot \mathrm{min}^{-1}$ & A & $38.1 \pm 6.1$ & - & $38.7 \pm 5.3$ & \\
\hline (body mass) & C & $39.1 \pm 5.6$ & $44.0 \pm 5.1^{* *}$ & - & 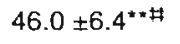 \\
\hline $\mathrm{ml} \cdot \mathrm{kg}^{-1} \cdot \mathrm{min}^{-1}$ & A & $49.4 \pm 5.8$ & & $49.5 \pm 5.0$ & \\
\hline (fat free mass) & C & $50.9 \pm 6.8$ & $56.5 \pm 4.9^{* *}$ & - & $57.7 \pm 6.1^{\star *}$ \\
\hline$W_{\max }$ & A & $243 \pm 43$ & - & $255 \pm 35$ & - \\
\hline (watt) & C & $252 \pm 51$ & $280 \pm 48^{* *}$ & - & $294 \pm 52^{* * 4}$ \\
\hline HRmax & A & $187 \pm 10$ & - & $186 \pm 9$ & \\
\hline (beats. $\min ^{-1}$ ) & C & $191 \pm 12$ & $190 \pm 9$ & - & $189 \pm 9$ \\
\hline \multirow[t]{2}{*}{ RER } & A & - & - & - & $=$ \\
\hline & C & $1.20 \pm 0.07$ & $1.20 \pm 0.05$ & - & $1.18 \pm 0.05$ \\
\hline
\end{tabular}

"significantly different from $T_{0}(" P<0.05, " P<0.005)$

"significantly different from $T_{8}\left({ }^{2} P<0.05, " p<0.005\right)$

there were no differences in $T_{0}$ data between study $A$ and $C$

Besides a strength-induced-increase in aerobic capacity, there are some considerations that may provide a rational that makes a true training induced aerobic effect likely. A possible explanation for a true increase in $\mathrm{VO} 2 \mathrm{max}$ is a fat-free mass related increase in VO2max. It has generally been thought that aerobic capacity during cycling is related to leg muscle size (6). Since the overall increase in fat-free mass will also consist of a hypertrophy of the musculature involved during cycling, it is possible that the increase in VO2max is caused by an increased amount of active muscle mass. Because no data are available to determine accurately the amount of active muscle mass during cycling, oxygen consumption is expressed in terms of $\mathrm{ml} \cdot \mathrm{kg}$ fat-free mass $^{-1} \cdot \min ^{-1}$. Data on VO2max relative to fat-free mass still reveal an increase in aerobic capacity (see Table 9.1), denoting that there was no fat-free mass related increase in $\mathrm{VO} 2 \mathrm{max}$. The similar increase in fat-free mass found in study-C without a corresponding increase in $\mathrm{VO} 2 \max$ confirms the latter finding. Therefore, it becomes more likely that the increase in $\mathrm{VO} 2 \mathrm{max}$ is truly due to an increased aerobic capacity of the muscle tissue. As previous mentioned, this is rarely found in conventional 
weight-training interventions. On the other hand, the workouts at the fitness club showed some similarity with circuit weight training. The training program was performed at a fairly rapid pace, completing 30 sets in approximately $50 \mathrm{~min}$. This resulted in relative short resting periods coupled with high-intensity performance during the weight-training exercises. This combination could have elevated heart rate similar as to what occurs in circuit weight training. During workouts at the fitness club average relative heart rate during the weight-training exercises was $43 \%$ of heart rate reserve (i.e., $63 \%$ maximal heart rate) (for data on resting, maximal and workout heart rates see chapter 6 ). In combination with the cycling exercise during the warming up and cooling down performed at approximately $65 \%$ of heart rate reserve (i.e., $78 \%$ maximal heart rate), the workout was performed on average at $50 \%$ of heart rate reserve (i.e., $67 \%$ maximal heart rate). Circuit weight-training interventions at comparable intensities have been shown to elicit increases in aerobic capacity. However, a change of a magnitude as found in the second intervention study $(+17.5 \%)$ is normally only noted after intense endurance exercise. A possible reason why VO2max may have increased to a somewhat higher degree was the initial low fitness level $\left(39.1 \mathrm{ml} \cdot \mathrm{kg}^{-1} \cdot \mathrm{min}^{-1}\right)$. This would seemly allow for greater absolute $\left(1 \cdot \mathrm{min}^{-1}\right)$ and relative (\%) increases than what is generally found with more active subjects (8).

Although the discussion presented above makes it more plausible that the second weight-training intervention induced a true increase in VO2max, it still does not explain the inconsistency in aerobic effect of weight training between the two interventions. Comparison of both interventions reveal that there were no major differences in weight-training intensity, initial fitness level, and increase in strength and fat-free mass. The only two differences between both studies were the duration of the intervention (12 versus $18 \mathrm{wk}$ ) and the cycling intensity of the warming up and cooling down (100-150 watt versus self selected intensity). Unpublished data on V O 2 max in study-C that were collected after 8 weeks of training $\left(\mathrm{T}_{8}\right.$, see Table 9.1) revealed that VO2max was already increased after 8 weeks. For this reason it can be concluded that the inconsistency in aerobic effect can not be ascribed to a duration difference between both interventions. Therefore, it is most likely that the increase in VO2max was primarily brought about by the cycling exercise performed during the 10 min warming up and cooling down.

\section{MAJOR CONCLUSIONS}

In summary, the major findings from the research presented in this thesis are:

1 An 18 wk weight-training program consisting of two 70 min sessions per week increased ADMR with approximately $10 \%$. Only $40 \%$ of the increase in ADMR could be attributed to the energy cost of the training program (chapter 8 ).

2 There was no weight-training-induced change in sleeping metabolic rate (chapter 3 and 8). However, weight training increased relative fat utilization during sleep in low fat oxidizers and vice versa for individuals displaying high pre-intervention lipid oxidation (chapter 3 ). 
3 Weight training induced on average an $8.5 \%$ decrease in the energy cost of intermittent weight-training exercises as well as in continuous arm cranking and cycling (chapter 6 ).

4 Comparing both intervention studies (study-A and study-C) revealed an inconsistent weight-training-induced effect on aerobic capacity. This inconsistency could not be explained by differences in the weight-training part of the workout. The major distinction between both exercise interventions was a different cycling intensity during the warming up and cooling down (chapter 2 and 6 ).

5 Weight training increased isokinetic muscular strength, and fat-frce mass and decreased fat mass (chapter 2,3,6 and 8). The increase in fat-free mass, however, is modified by body build meaning a larger absolute $(\mathrm{kg})$ and relative $(\%)$ increase in fat-free mass in more solidly built subjects (chapter 2 ).

6 Intra-individual day-to-day variation in energy expenditure during weight training did not differ from variation during arm cranking or cycling (chapter 4).

7 Energy expenditure during weight training can be predicted accurately using a nonincremental calibration protocol provided that the energy expenditure-heart rate relationship is determined at a comparable intensity. Higher heart rates during the workout compared to the heart rate during the calibration session result in an overestimated predicted energy expenditure whereas lower workout heart rates underestimated the predicted energy expenditure (chapter 7 ).

8 Energy expenditure estimated from energy intake measured by a three-day food record substantially underestimates (21-34\%) ADMR as determined with the doubly labelled water technique (chapter 8 ).

\section{REFERENCES}

I Ballor, D. L., V. L. Katch, M. D. Becque, C. R. Marks. Resistance weight training during caloric restriction enhances lean body weight maintenance. Am. J. Clin. Nutr: 47:19-25, 1988

2 Bouten, C. V. C., W. P. H. G. Verboeket-van de Venne, K. R. Westerterp, M. Verduin, J. D. Janssen. Daily physical activity assessment: comparison between movement registration and doubly labeled water. J. Appl. Physiol. 81(2):10191026, 1996.

3 Bouten, C. V. C., K. R. Westerterp, M. Verduin, J. D. Janssen, "Assesment of daily physical activity by registration of body movements," in Human Biology. Maastricht: University Maastricht, 1995, pp. chapter 5.

4 Fuller, N. J., S. A. Jebb, M. A. Laskey, W. A. Coward, M. Elia. Four-component model for the assessment of body composition in humans: comparison with alternative methods, and evaluation of the density and hydration of fat -free mass. Clin. Sci. 82:687-693, 1992.

5 Goran, M. I., E. T. Poehlman. Endurance training does not enhance total energy expenditure in healthy elderly persons. Am. J. Physiol. 263(26):E950-E957, 1992. 
6 Hickson, R. C., M. A. Rosenkoetter, M. M. Brown. Strength training effects on aerobic power and short-term endurance. Med. Sci. Sports Exerc. 12:336-339, 1980.

7 Hill, J. O., D. G. Slundt, T. S. Sbrocco, et al. Evaluation of an alternating-calorie diet with and without exercise in the treatment of obesity. Am. J. Clin. Nutr. 50:248-254, 1989.

8 McCarthy, J. P., J. C. Agre, B. K. Graf, M. A. Pozniac, A. C. Vailas. Compatibility of addaptive responses with combining strength and endurance training. Med. Sci. Sports Exerc. 27(3):429-436, 1995.

9 Melby, C. L., T. Tincknell, W. D. Schmidt. Energy expenditure following a bout of non-steady state resistance exercise. J. Sports Med. Phys. Fitness 32:128-135, 1992.

10 Modlesky, C. M., K. J. Cureton, R. D. Lewis, B. M. Prior, M. A. Sloniger, D. A. Rowe. Density of the fat-free mass and estimates of body composition in male weight trainers. J. Appl. Physiol. 80(6):2085-2096, 1996.

11 Molé, P. Impact of energy intake and exercise on resting metabolic rate. Sports Med. 10:72-87, 1990.

12 Morgan, D. W., P. E. Martin, G. S. Krahenbuhl. Factors affecting running economy. Sports Med. 7:310-330, 1989.

13 Murgatroyd, P., W. Coward. An improved method for estimating changes in whole-body fat and protein mass in man. Brit. J. Nutr. 62:311-314, 1989.

14 Noakes, T. D. Implications of exercise testing for prediction of athletic performance: a contemporary perspective. Med. Sci. Sports Exerc. 20(4):319-330, 1988.

15 Poehlman, E. T., C. L. Melby, M. I. Goran. The impact of exercise and diet restriction on daily energy expenditure. Sports Med. 11:78-101, 1991.

16 Siri, W. E., Body composition from fluid spaces and density: analysis of methods., in Techniques for measuring body composition, Brozek, J. and A. Henschel, Eds. Washington, DC: Natl. Acad. Sci., 1961, pp. 223-244.

17 Stone, M. H., S. J. Fleck, N. Travis Triplet, W. J. Kraemer. Health and performance-related potential of resistance training. Sports Med. 11(4):210-231, 1991.

18 Verstappen, F. T. J., L. M. L. A. Etten van, K. R. Westerterp. The use of heart rate monitoring to predict energy expenditure during weight-training. Med. Sci. Sports Exerc. 27(5):S17, 1995. 


\section{Samenvatting}

Een studie naar de gevolgen van fysieke training/inspanning op het energiegebruik lijkt in eerste instantie overbodig. Algemeen wordt aangenomen dat door toename van lichamelijke activiteit het energiegebruik omhoog gaat. In een tijdperk waarin overgewicht (overmaat aan lichaamsvet) niet uitsluitend ongewenst is vanwege een mogelijk verhoogd gezondheidsrisico maar eveneens vanwege nadelige cosmetische consequenties, bestaat dan ook een groot aanbod aan bewegingsprogramma's die zich richten op het terugbrengen van overgewicht. Maar wat is er daadwerkelijk bekend over het effect van training op het energiegebruik? Het grootste deel van het wetenschappelijk onderzoek op dit gebied beperkt zich tot de directe energiekosten van de toegevoegde activiteit. Maar wat gebeurt er met het dagelijkse energiegebruik? Wordt de extra inspanning gedurende de dag misschien gecompenseerd of worden de mensen juist actiever gedurende de rest van de dag? $\mathrm{Er}$ is nog maar zeer weinig onderzoek verricht naar het effect van training op het dagelijkse (24-uur) energiegebruik, terwijl dit energiegebruik samen met de energie-inname nu juist de energiebalans bepalen. De oorzaak van de geringe omvang van onderzoek naar het effect op het dagelijkse energiegebruik is een gevolg van de beperkte mogelijkheden (technisch en financieel) om het energiegebruik in de normale leefsituatie te meten.

Het energiegebruik voor lichamelijkc activiteit -en dus ook voor de trainingsarbeidvormt echter maar een relatief klein deel van het totale dagelijkse energiegebruik (15-30\%). Het resterende deel komt voor rekening van de basale lichaamsfuncties (de $\mathrm{zgn}$. ruststofwisseling, $60-75 \%)$ en de verwerking van voedsel $(\approx 10 \%)$. Een additioneel trainingsgeïnduceerd effect op deze andere componenten van het energiegebruik beïnvloedt uiteraard ook het dagelijkse energiegebruik. Er zijn bijvoorbeeld aanwijzingen dat training de ruststofwisseling zou kunnen verhogen. Aangezien de ruststofwisseling het grootste deel van het dagelijkse energiegebruik vormt, zal een relatief kleine verandering een substantiee] effect hebben op het totale energiegebruik.

Zoals eerder vermeld bestaat er een uitgebreid aanbod aan uiteenlopende bewegingsprogramma's. Onderzoek naar het effect op het energiegebruik heeft zich tot nu toe beperkt tot vormen van duursport, zoals joggen en fietsen. Naast duursport zijn er vele trainingsvormen van een minder continue karakter, d.w.z. die inspanningsperioden afwisselen met rustperioden (interval-sporten). Fitness-training is zo'n vorm van interval-training die de laatste jaren een toenemende populariteit geniet. Bij fitness-training wordt gebruik gemaakt van gewichten (halters, dumbbells) om de weerstand tijdens het bewegen te verhogen volgens het overload-principe. De intervalbelasting bestaat uit korte periodes van inspanning ( $\pm 30 \mathrm{sec}$ ) afgewisseld met rustperiodes. Van fitness-training is echter bekend dat het energiegebruik tijdens training minder wordt verhoogd dan bijvoorbeeld tijdens joggen of fietsen. Daarentegen zou fitness-training wel een grotere toename in vetvrije massa bewerkstelligen. Deze vetvrije-massa vertoont niet alleen een positief verband met een groot aantal fitheidsparameters, maar vormt tevens de belangrijkste determinant van de ruststofwisseling, de grootste component van het dagelijkse energiegebruik. Het doel van het 
eerste onderzoek (studie-A) was dan ook na te gaan of een fitness trainingsprogramma de ruststofwisseling beïnvloedt.

Praktijkervaring leerde dat er grote verschillen in de fitness-geïnduceerd toename in vetvrije massa kunnen bestaan tussen personen mogelijk een gevolg van verschillen in lichaamsbouw. Omdat deze verschillen het aan de vetvrije massa gerelateerde potentiele trainingseffect op de ruststofwisseling zou kunnen beïvloeden, vormde lichaamsbouw een afzonderlijk onderdeel in deze studie (hoofdstuk 2). Lichaamsbouw werd gedefinieerd als de hoeveelheid vetvrije massa gecorrigeerd voor lichaamslengte en vetmassa. Tijdens het samenstellen van de onderzoekspopulatie werden uitsluitend proefpersonen geselecteerd die getypeerd werden door een solide lichaamsbouw (hoge relatieve vetvrije massa, $n=11$ ) dan wel een tengere lichaamsbouw (lage relatieve vetvrije massa, $n=10$ ).

$\mathrm{Na}$ een trainingsperiode van twaalf weken waarin de deelnemers twee keer per week trainden, bedroeg de gemiddelde toename in vetvrije massa $0,9 \mathrm{~kg}$, terwijl de vetmassa met $2,1 \mathrm{~kg}$ afnam. Bij een vergelijking van de twee sub-groepen, vertoonde de groep met een solide lichaamsbouw een grotere absolute (1.6 versus $0.3 \mathrm{~kg}$; $\mathrm{P}<0.01)$ en relatieve $(2,3$ vs $0,5 \% ; \mathrm{P}<0,01)$ toename van vetvrije massa. Er was geen verschil in afname van de vetmassa tussen beide groepen $(-2,4 \mathrm{vs}-1,7 \mathrm{~kg} ;-11,3$ vs $-10,5 \%$ ). Verder verschilden beide groepen niet in krachttoename (gemid-deld 13,7\%). Het uithoudingsvermogen gemeten tijdens een opklimmende fietstest veranderde niet.

Om het effect van training op de ruststofwisseling te bepalen werd vóór en na de trainingsperiode het energiegebruik tijdens een overnachting in een respiratiekamer gemeten (hoofdstuk 3). Uit de resultaten bleek dat een fitness-programma van twaalf weken geen effect had op het energiegebruik tijdens de slaap (ruststofwisseling).

$\mathrm{Nu}$ uit voorgaande studie was gebleken dat fitness-training geen effect had op de ruststofwisseling, resteerde de vraag of het energetische effect van fitness-training zich werkelijk uitsluitend zou beperken tot de energiekosten van de toegevoegde training. De enige vier studies die tot dan het overall-effect van een trainings-interventie op het dagelijkse energiegebruik daadwerkelijk gemeten hadden gaven tegenstrijdige resultaten. Uit één studie bleek dat de energiekosten van de training volledig gecompenseerd werd door een afname in spontane lichamelijke activiteit. De andere drie studies gaven aan dat de totale toename beduidend hoger is dan de energiekosten van het toegevoegde trainingsprogramma.

Een factor die het effect van training op het energiegebruik nog verder compliceert is het effect van training op het energiegebruik tijdens inspanning. Getrainden zouden minder energie nodig hebben voor dezelfde arbeid. Dit zou een tegenstrijdig trainingseffect zijn aangezien het verhogen van het energiegebruik een belangrijk doel is van de bewegingsprogramma's.

Alvorens nu een studie te starten waarbij zowel gekeken werd naar het effect van een toegevoegde training op het dagelijkse energiegebruik als naar het effect van getraindheid op het energiegebruik tijdens inspanning, moest er een methode gevonden worden om het energiegebruik tijdens een fitness-training betrouwbaar en reproduceerbaar te meten. In een tweede studie (studie-B), waaraan 10 getrainde proefpersonen deelnamen, was het mogelijk deze meting met een reproduceerbaarheid van 
ongeveer $\pm 3 \%$ uit te voeren (hoofdstuk 4). Deze reproduceerbaarheid was vergelijkbaar met de reproduceerbaarheid van energiemetingen tijdens duurinspanning. Tijdens de fitness-meting werd gebruik gemaakt van een nauwgezet gestandaardiseerd protocol waarbij het tempo van de bewegingen en de duur van de inspannings- en rustperiodes gedicteerd werd door een computersignaal.

Om het effect van fitness-training op zowel het energiegebruik tijdens training als op het totale energiegebruik te bestuderen volgden achttien ongetrainde mannen een achttien weken durende trainingsinterventie, waarin twee fitness-trainingen per week moesten worden uitgevoerd (studie-C).

Data over het energiegebruik tijdens de gestandaardiseerde trainingen lieten zien dat na achttien weken fitness-training het energiegebruik, zowel tijdens de specificke fitness-oefeningen als tijdens duurinspanning, met respectievclijk 9,6 en $7,5 \%$ alnam (hoofdstuk 6).

Om een gedetailleerd beeld te krijgen van het effect van een fitness-training op het totale dagelijkse energiegebruik, werden gegevens verzameld over de volgende componenten van het energiegebruik (hoofdstuk 8). Het totale energiegebruik werd over een periode van twee weken gemeten met behulp van tweevoudig gemerkt water. De ruststofwisseling werd wederom gemeten in een respiratiekamer, terwijl het energiegebruik tijdens lichamelijke activiteit in twee afzonderlijke onderdelen werd opgesplitst. Het energiegebruik tijdens de fitness-trainingen in het sportcentrum werd berekend op basis van de trainingshartfrequenties (hoofdstuk 7), terwijl de spontane lichamelijke activiteit werd geregistreerd met behulp van een versnellingsopnemer. Er werd aangenomen dat de verwerking van voedsel niet zou veranderen en $10 \%$ van het totale energiegebruik zou bedragen. $\mathrm{Na}$ achttien weken nam het dagelijkse energiegebruik toe met 9,5\% (hoofdstuk 8). Evenals in de eerste studie had training geen effect op de ruststofwisseling. Verder was er geen verandering van spontane lichamelijke activiteit waarneembaar. De energiekosten van de toegevoegde trainingsarbeid vormden echter maar $40-50 \%$ van de totale toename in energiegebruik. Er werd geen verandering in spontane lichamelijke aktiviteit waargenomen. De meest voor de hand liggende verklaring voor de discrepantie in het toegenomen energiegebruik, lijkt dan ook een combinatie van een onderschatte ruststofwisseling en een onderschat energiegebruik tijdens de trainingen.

Tijdens deze studie werd eveneens het effect van fitness-training op kracht en uithoudingsvermogen bepaald. De toename in kracht was vergelijkbaar met de eerste interventiestudies. De resultaten van de fietstest vertoonden echter een duidelijk ander beeld. Terwijl in de eerste studie er geen verandering in uithoudingsvermogen werd waargenomen, vertoonden de deelnemers aan de laatste studie een $17 \%$ toename in aëroob vermogen. Dit verschil tussen beide studies is mogelijk een gevolg van een verschil in warming-up methode die werd gebruikt tijdens beide studies. In studie-A mochten de deelnemers niet hoger gaan dan 150 watt, terwijl in studie-C ze hier volledig vrij in werden gelaten.

Op basis van de resultaten van beide interventie-studies kan geconcludeerd worden dat fitness-training, ondanks een gemiddcld energiegebruik en een energie sparend 
effect tijdens inspanning, een geschikte trainingsmethode is om het dagelijkse energiegebruik te verhogen. 


\section{Abbreviations}

\begin{tabular}{ll} 
\% fat & percentage body fat \\
$\Delta$ & delta (difference) \\
$1 \mathrm{RM}$ & one repetition maximum \\
${ }^{2} \mathrm{H}_{2} \mathrm{O}$ & deuterium \\
${ }^{2} \mathrm{H}_{2}{ }^{18} \mathrm{O}$ & doubly labelled water \\
$\mathrm{ADMR}$ & average daily metabolic rate \\
$\mathrm{Bf}$ & breathing frequency \\
$\mathrm{BMI}$ & body mass index \\
$\mathrm{BV}$ & body volume \\
$\mathrm{BW}$ & body weight \\
$\mathrm{cm}$ & centimetre \\
$\mathrm{CD} 5$ & last five minutes of the cooling-down phase \\
$\mathrm{CO}$ & carbon dioxide \\
$\mathrm{CONT}$ & control group \\
$\mathrm{CWT}$ & circuit weight training \\
$\mathrm{DIT}$ & diet-induced thermogenesis \\
EE & energy expenditure \\
$\mathrm{EEact}$ & energy expenditure during spontaneous activity \\
$\mathrm{EEhigh}$ & the average energy cost of the two higher intensity sessions \\
$\mathrm{EElow}$ & the average energy cost of the two lower intensity sessions \\
$\mathrm{EI}$ & energy intake \\
EEex & energy expenditure during exercise \\
EPOC & excess post-exercise oxygen consumption \\
EQ & equation \\
EXER & exercise group \\
FFM & fat-free mass \\
FFMI & fat-free mass index \\
FM & fat mass \\
FMI & fat mass index \\
$\mathrm{g}$ & gram \\
$\mathrm{h}$ & hour \\
$\mathrm{H} 1$ or H2 & first or second higher intensity session \\
$\mathrm{H} 1 / 2$ & average of higher intensity sessions \\
$\mathrm{HR}$ & heart rate \\
$\mathrm{J}$ & joule \\
$\mathrm{kJ}$ & kilojoule \\
min & minute \\
$\mathrm{MJ}$ & megajoule \\
$\mathrm{kg}$ & kilogram \\
$\mathrm{L} 1$ or L2 & first or second lower intensity session \\
L1/2 & average of lower intensity sessions \\
& \\
\hline
\end{tabular}




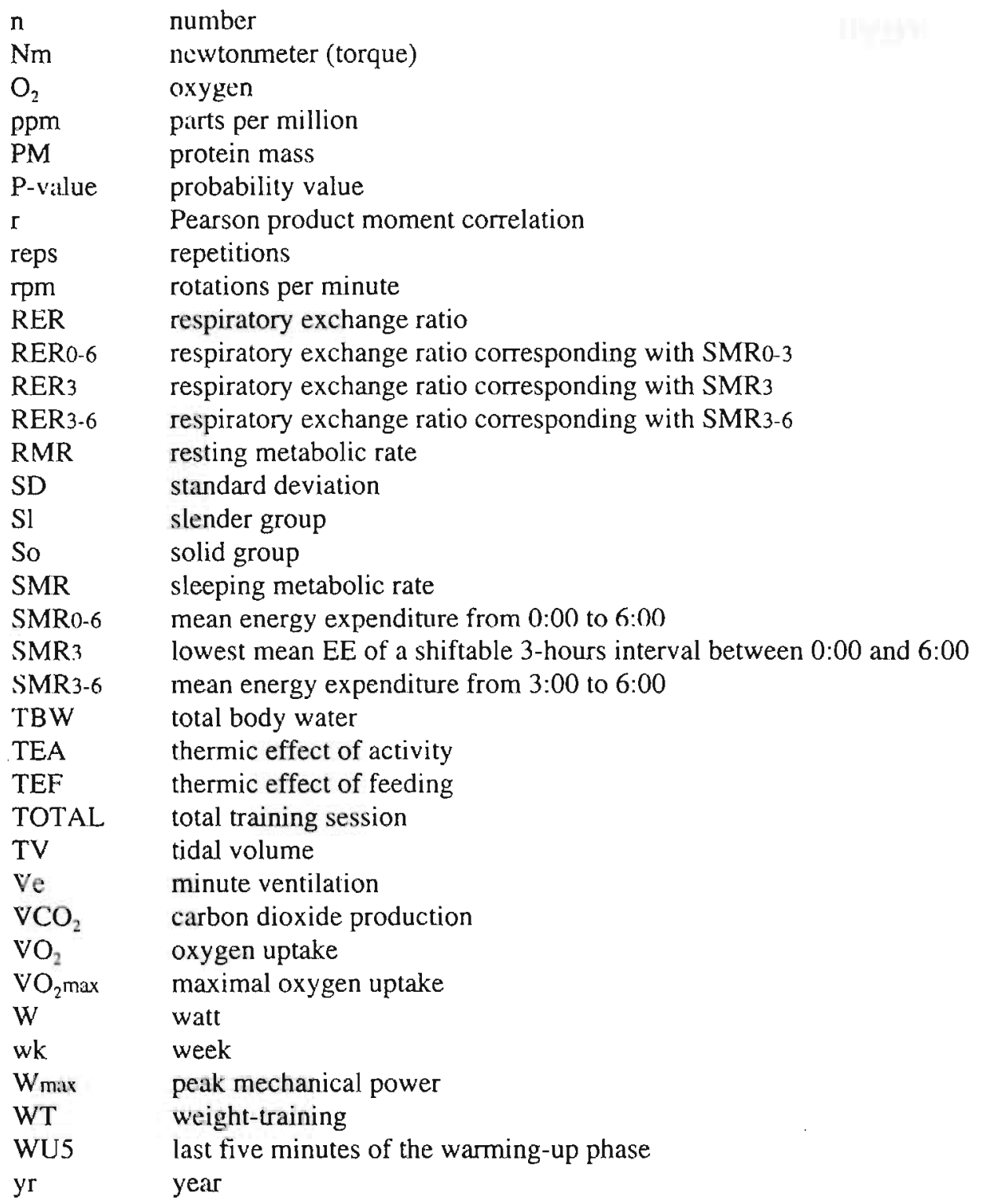




\section{Nawoord}

Een promotieproject komt voort uit een aantal vragen waarop je graag een antwoord zou willen hebben. Om dit zo efficiënt mogelijk en met relatief weinig problemen te laten verlopen probeer je alles zo goed mogelijk voor te bereiden en alternatieven voor handen te hebben om adequaat te kunnen reageren wanneer zich problemen voordoen. Dat niet alles kan worden voorzien -en dit geldt zeer zeker voor humaan interventieonderzoek- is evident. Ondanks alle moeite zijn er dan ook momenten waarop je naar aanleiding van een volgende tegenvaller jezelf afvraagt: 'waar heb ik dit nu weer aan te danken?'. Merkwaardigerwijze realiseer je je op zo'n moment niet dat dit een vraag is waarmee een proefschrift vaak wordt afgesloten.

Je kunt natuurlijk op safe spelen door iedereen te bedanken die vindt dat hij/zij, op welke wijze dan ook, een bijdrage heeft geleverd aan het tot stand komen van dit proefschrift. Dit zou echter betekenen dat ik de gelegenheid voorbij laat gaan om de mensen te bedanken waarvan ik vind dat ze een speciale bijdrage aan dit onderzoek hebben geleverd. De motivatie om een bijdrage te leveren kwam voort uit professionele interesse, persoonlijke belangen, collegialiteit dan wel uit vriendschap. Zelden had ik de indruk dat de betrokkenheid zich beperkte tot één van deze factoren, en was er eerder sprake van accentverschillen.

-Om te beginnen wil ik mijn twee begeleiders Klaas Westerterp, Frans Verstappen en mijn promotor Wim Saris, evenals de bijbehorende vakgroepen Humane Biologie en Bewegingswetenschappen, bedanken voor hun bijdrage in kennis, motivatie en faciliteiten.

-Naast begeleiding heb je op je werkplek ook te maken met collega's en in het bijzonder met kamergenoten. Kamergenoten lijken wel wat op familieleden: je kunt ze niet kiezen, je krijgt ze. Mijn kamergenoten vertoonden echter nog meer overeenkomsten met familieleden: er werd zelden over het werk gepraat. Asker en Jos, bedankt voor de gezelligheid en de manier waarop jullie -ieder op zijn eigen kenmerkende wijze- een motivatie waren om dit proefschrift (snel?) af te ronden.

-Humaan onderzoek is alleen maar mogelijk als mensen bereid zijn deel te nemen. Bij geen van de onderzoeken ontvingen de proefpersonen een financiële vergoeding terwijl er een aanzienlijk beroep werd gedaan op hun vrije tijd. Hopelijk hebben jullie deze samenwerking even prettig en boeiend gevonden als ik.

-Voor de data collectie en verwerking wordt gebruik gemaakt van apparatuur. Dat dit niet altijd even vloeiend verloopt ligt voor de hand. Gelukkig waren er collega's als Harry Wandler, Loek Wouters en Paul Schoffelen die ten alle tijden bereid waren hulp te bieden. Ook de technische en instrumentele dienst wil ik bedanken voor hun bijdrage in het succesvol uitvoeren van dit project.

-De trainingsprogramma's konden alleen maar worden uitgevoerd dankzij de voortreffelijke medewerking van de sportschoolhouders en het personeel van de sportcentra INTERFIT (Meerssen) en TOPFIT (Maastricht).

-Tijdens het laatste experiment had ik hulp van Bart Boon (student-assistent) en Ankie Verlouw (stage-student). Zonder hun hulp was de strakke planning van het onderzoek 
en praktische uitvoering niet mogelijk geweest. Ik was met name aangenaam verrast door de wijze waarop Bart in de weekenden, vaak al vroeg in de ochtend (niet bepaald zijn beste tijdstip), zonder al te veel morren de groep van controle-personen met strakke hand bestierde.

-Het proefschrift is voor een groot deel geschreven gedurende mijn aanstelling als docent aan de St Michaël VSO, Maastricht. De leiding en collega's van deze school wil ik bedanken voor de volledige medewerking en de aangename afwisseling die deze baan mij bood.

- Tijdens experimenten blijft er weinig tijd over om sociale kontakten te onderhouden. Ik ben dan ook heel blij met het begrip dat ik ontving van vrienden en vriendinnen -in het bijzonder van Jose- voor het verwaarlozen van mijn sociale en maatschappelijke verplichtingen. 


\section{Curriculum vitae}

3 mei 1958 Geboren te Bunde

1976 HAVO diploma aan het St. Maartenscollege, Maastricht

1978-1979 Militaire dienstplicht, Venlo

1983 Examen Haagse Academie voor Lichamelijke Opvoeding, Den Haag

1983-1987 Docent Lichamelijke Opvoeding:

Rijnlands Lyceum, Sassenheim (1983-1984)

Nijmeegse Scholengemeenschap, Nijmegen (1985-1987)

1991 Doctoraal diploma gezondheidswetenschappen aan de Rijksuniversiteit Limburg, Maastricht

1991-1994 Toegevoegd onderzoeker bij het Instituut voor Bewegingswetenschappen, Rijksuniversiteit Limburg, Maastricht

1995 Assistent in opleiding bij de vakgroep Humane Biologie, Rijksuniversiteit Limburg, Maastricht

1996 Docent Lichamelijke Opvoeding: St. Michaël VSO, Maastricht 


\section{Publications}

\section{Articles}

- Van Etten, L.M.L.A., F.T.J. Verstappen, K.R. Westerterp. Effect of body build on weight-training-induced adaptations in body composition and muscular strength. Med. Sci. Sports Exerc. 26(4):515-521, 1994.

- Van Etten, L.M.L.A., K.R. Westerterp, F.T.J. Verstappen. Effect of weight-training on energy expenditure and substrate utilization during sleep. Med. Sci. Sports Exerc. 27(2):188-193, 1995.

- Van Etten, L.M.L.A., K.R. Westerterp, J.W.E. Verlouw, F.T.J. Verstappen. Invloed van fitnesstraining. Voeding 57(5):31, 1996.

- Van Etten, L.M.L.A., K.R. Westerterp, F.T.J. Verstappen. Daily variation in energy expenditure during weight-training versus continuous arm cranking and cycling. Int. J. Sports Med. 17(7):497-503, 1996.

- Van Etten, L.M.L.A., K.R. Westerterp, F.T.J. Verstappen, B.J.B. Boon, W.H.M. Saris. Effect of an 18-week weight-training program on energy expenditure and physical activity. J. Appl. Physiol. 82(1): In Press. 1976.

- Van Etten, L.M.L.A., F.T.J. Verstappen, K.R. Westerterp. The energy expenditureheart rate relationship during weight-training. submitted.

- Van Etten, L.M.L.A., F.T.J. Verstappen, K.R. Westerterp, B.J.B. Boon, W.H.M. Saris. Determinants of energy expenditure during weight-training. submitted.

- Van Etten, L.M.L.A., F.T.J. Verstappen, K.R. Westerterp, B.J.B. Boon, W.H.M. Saris. Effect of 18 weeks of weight-training on exercise economy. submitted.

\section{Abstracts}

- Westerterp, K.R., L.M.L.A. Etten van, G.A.L. Meijer, F.T.J. Verstappen. Fasting substrate utilization before and after strength and endurance training. Int. J. Obes. 16 suppl 1:58, 1992.

- Westerterp, K.R., L.M.L.A. Etten van, G.A.L. Meijer, F.T.J. Verstappen. Fat-free mass and sleeping metabolic rate before and after endurance and strength training. Int. J. Obes. 16 suppl 1:58, 1992.

- Van Etten, L.M.L.A., F.T.J. Verstappen, K.R. Westerterp. Effect of body-build on weight-training induced adaptations in body composition, strength and aerobic power. Med. Sci. Sports Exerc. 25(5):S58, 1993.

- Westerterp, K.R., L.M.L.A. Etten van, F.T.J. Verstappen. Weight-training modified substrate utilization during sleep. Med. Sci. Sports Exerc. 25(5):S191, 1993.

- Van Etten, L.M.L.A., K.R. Westerterp, F.T.J. Verstappen. Intra-individual variability in energy expenditure during weight-training versus arm- and leg-cycling. Med. Sci. Sports Exerc. 27(5):S88, 1995. 
- Verstappen, F.T.J., L.M.L.A. Etten van, K.R. Westerterp. The use of heart rate monitoring to predict energy expenditure during weight-training. Med. Sci. Sports Excrc. 27(5):S17, 1995.

- Van Etten, L.M.L.A., K.R. Westerterp, F.T.J. Verstappen. 18-wks weight-training decreased energy expenditure and heart rate during continuous and intermittent exercise. Med. Sci. Sports Exerc. 28(5):S86, 1996.

- Verstappen, F.T.J., L.M.L.A. Van Etten, K.R. Westerterp. State of training: a determinant of energy expenditure during weight-training. Med. Sci. Sports Exerc. 28(5):S128, 1996.

- Westerterp, K.R., L.M.L.A. Van Etten, F.T.J. Verstappen. Effect of an 18-wk weight-training program on daily energy expenditure, sleeping metabolic rate and physical activity. Med. Sci. Sports Exerc. 28(5):S32, 1996.

- Van Etten, L.M.L.A., B.J.B. Boon, K.R. Westerterp, F.T.J. Verstappen. Effect of exercise mode on blood lactate during isoenergetic intermittent weight-training versus continuous cycling. Med. Sci. Sports Exerc. In Press. 


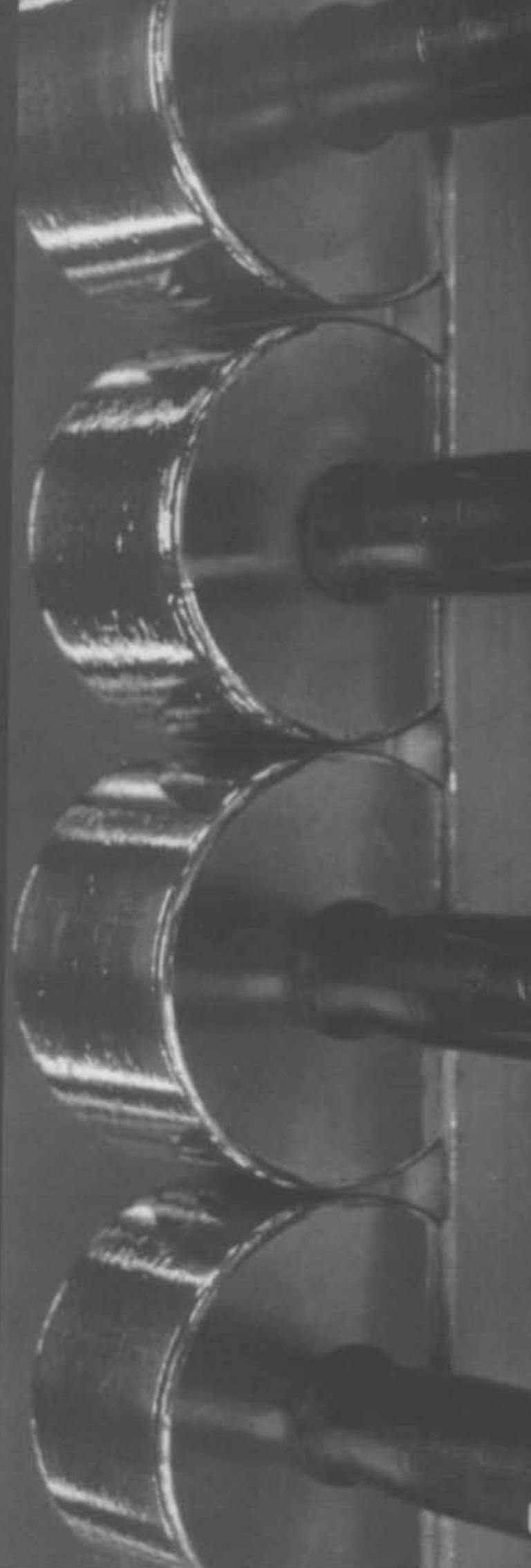

